\title{
GEOMETRIC EISENSTEIN SERIES: TWISTED SETTING
}

\author{
SERGEY LYSENKO
}

\begin{abstract}
Let $G$ be a simple simply-connected group over an algebraically closed field $k, X$ be a smooth connected projective curve over $k$. In this paper we develop the theory of geometric Eisenstein series on the moduli stack $\operatorname{Bun}_{G}$ of $G$-torsors on $X$ in the setting

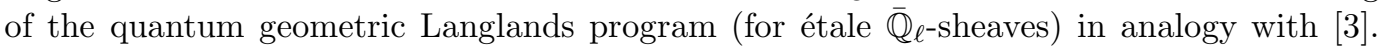
We calculate the intersection cohomology sheaf on the version of Drinfeld compactification in our twisted setting. In the case $G=\mathrm{SL}_{2}$ we derive some results about the Fourier coefficients of our Eisenstein series. In the case of $G=S L_{2}$ and $X=\mathbb{P}^{1}$ we also construct the corresponding theta-sheaves and prove their Hecke property.
\end{abstract}

\section{Contents}

1. Introduction

2. Main results

3. Principal geometric Eisenstein series

3.1. Definitions

3.2. Hecke functors

3.3. Proof of Theorem 2.2

3.4. Proof of Theorem 2.1

3.5. Some gradings

3.6. Towards the functional equation

3.7. Action of $\operatorname{Bun}_{Z(G)}$

4. Parabolic geometric Eisenstein series

4.1. Definitions

4.2. Hecke functors for $M$

4.3. Geometric restriction functors

4.4. Proof of Theorem 2.4

4.5. Description of $\mathrm{IC}_{\zeta}$

5. Proof of Theorem 4.1

5.1. Zastava spaces

5.2. Main technical step

5.3. Proof of Theorem 4.1

5.4. Induction

6. Composing Eisenstein series

7. The case of $G=\mathrm{SL}_{2}$ 
7.2. Fourier coefficients 54

7.3. Constant term of Eis $\quad 60$

7.4. Some special sheaves $\quad 62$

\begin{tabular}{ll|l|} 
Appendix A. Proof of Theorem [2.6 & 68 \\
\hline
\end{tabular}

\begin{tabular}{ll|} 
A.1. & 68 \\
\hline
\end{tabular}

\begin{tabular}{ll} 
References & 69 \\
\hline
\end{tabular}

\section{INTRODUCTION}

1.0.1. In this paper we develop an analog of the theory of geometric Eisenstein series from [3] for the twisted geometric Langlands correspondence. Here 'twisted' refers to the quantum Langlands correspondence (as outlined in [12, 13, 25]) with the quantum parameter being a root of unity. In the case of a split torus the corresponding geometric setting was proposed in [17].

The conjectural extension of the Langlands program for metaplectic groups was proposed by M. Weissman in ([28, 29], see also [14, 15]). In his approach the metaplectic group is a central extension of a reductive group by a finite cyclic group coming from Brylinski-Deligne theory [5]. Our approach is a geometrization of this evolving program.

For motivations, take $k=\mathbb{F}_{q}$. Let $X$ be a smooth projective curve over $k, G$ be a split reductive group. Let $\mathbb{A}$ be the adeles ring of $F=k(X), \mathcal{O} \subset \mathbb{A}$ be the integer adeles. Let $n \geq 1, n \mid q-1$. A Brylinski-Deligne extension of $G$ by $K_{2}$ gives rise to an extension $1 \rightarrow \mu_{n}(k) \rightarrow \tilde{G} \rightarrow G(\mathbb{A}) \rightarrow 1$ together with its splittings over $G(F)$ and $G(\mathcal{O})$. Pick an injective character $\bar{\zeta}: \mu_{n}(k) \rightarrow \overline{\mathbb{Q}}_{\ell}^{*}$. The global nonramified Langlands program for $\tilde{G}$ aims to find the spectral decomposition of the space $\operatorname{Funct}_{\bar{\zeta}}(G(F) \backslash \tilde{G} / G(\mathcal{O}))$ of $\overline{\mathbb{Q}}_{\ell}$-valued functions that change by $\bar{\zeta}$ under the action of $\mu_{n}(k)$.

A fundamental tool for this program is the theory of Eisenstein series. Let $M \subset P \subset G$ be a Levi subgroup of a parabolic subgroup of $G$. By restriction the above yield the metaplectic extensions $\tilde{M}$ (resp., $\tilde{P}$ ) of $M(\mathbb{A})$ (resp., $P(\mathbb{A})$ ). One gets the diagram of projections

$$
M(F) \backslash \tilde{M} / M(\mathcal{O}) \stackrel{\mathfrak{q}}{\leftarrow} P(F) \backslash \tilde{P} / P(\mathcal{O}) \stackrel{\mathfrak{p}}{\rightarrow} G(F) \backslash \tilde{G} / G(\mathcal{O}) .
$$

For a compactly supported function $f \in \operatorname{Funct}_{\bar{\zeta}}(M(F) \backslash \tilde{M} / M(\mathcal{O}))$ the associated Eisenstein series (up to a normalization factor) is $\mathfrak{p} ! \mathfrak{q}^{*} f$.

We study a geometric analog of this construction. We work with étale $\overline{\mathbb{Q}}_{\ell}$-sheaves to keep a close relation with the more classical Langlands program for the corresponding metaplectic groups.

1.0.2. Let $k$ be an algebraically closed field, $G$ be a simple, simply-connected group over $k$. In this case there is a canonical Brylinski-Deligne extension of $G$ by $K_{2}$ (the others are obtained from it up to isomorphism as its multiples).

Let $X$ be a smooth projective connected curve over $k$. Let $\operatorname{Bun}_{G}$ be the stack of $G$ torsors on $X$. Pick $n \geq 1$. We introduce some $\mu_{N^{-}}$gerb $\widetilde{\operatorname{Bun}_{G}} \rightarrow \operatorname{Bun}_{G}$ with $N=2 \check{h} n$, 
it comes from the canonical Brylinski-Deligne extension of $G$ by $K_{2}$. Here $\check{h}$ is the dual Coxeter number for $G$. We pick an injective character $\zeta: \mu_{N}(k) \rightarrow \overline{\mathbb{Q}}_{\ell}^{*}$ and consider the derived category $\mathrm{D}_{\zeta}\left(\widetilde{\operatorname{Bun}}_{G}\right)$ of étale $\overline{\mathbb{Q}}_{\ell}$-sheaves on $\widetilde{\operatorname{Bun}_{G}}$, on which $\mu_{N}(k)$ acts by $\zeta$. To these metaplectic data there corresponds a dual group $\check{G}_{n}$ defined in [9]. The category $\operatorname{Rep}\left(\check{G}_{n}\right)$ of finite-dimensional representations of $\check{G}_{n}$ acts on $\mathrm{D}_{\zeta}\left(\widetilde{\operatorname{Bun}}_{G}\right)$ by Hecke functors. The twisted geometric Langlands problem in this case is to construct Hecke eigen-sheaves in $\mathrm{D}_{\zeta}\left(\widetilde{\operatorname{Bun}_{G}}\right)$ (or even a spectral decomposition thereof).

Let $P \subset G$ be a parabolic subgroup, $M$ its Levi factor. We similarly get a $\mu_{N^{-}}$gerb $\widetilde{\operatorname{Bun}}_{M} \rightarrow \operatorname{Bun}_{M}$ and the derived category $\mathrm{D}_{\zeta}\left(\widetilde{\operatorname{Bun}}_{M}\right)$. As in [9], one has the corresponding Levi subgroup $\check{M}_{n} \subset \check{G}_{n}$, and $\operatorname{Rep}\left(\check{M}_{n}\right)$ acts on $\mathrm{D}_{\zeta}\left(\widetilde{\operatorname{Bun}}_{M}\right)$ by Hecke functors.

The Eisenstein series from Section 1.0.1 admits an immediate geometrization

$$
\operatorname{Eis}^{\prime}: \mathrm{D}_{\zeta}\left(\widetilde{\operatorname{Bun}}_{M}\right) \rightarrow \mathrm{D}_{\zeta}\left(\widetilde{\operatorname{Bun}}_{G}\right)
$$

However, Eis' does not commute with the Verdier duality and may be improved replacing $\widetilde{\operatorname{Bun}}_{P}$ by the relative Drinfeld's compactification $\widetilde{\mathrm{Bun}}_{\tilde{P}}$ along the fibres of the projection $\operatorname{Bun}_{P} \rightarrow \operatorname{Bun}_{G}$ as in [3].

We define the corresponding compactified Eisenstein series functor Eis : $\mathrm{D}_{\zeta}\left(\widetilde{\mathrm{Bun}_{M}}\right) \rightarrow$ $\mathrm{D}_{\zeta}\left(\widetilde{\operatorname{Bun}}_{G}\right)$ and study its properties. It is defined using a twisted version $\mathrm{IC}_{\zeta}$ of the IC-sheaf of $\widetilde{\operatorname{Bun}}_{P}$. One of our main results is the description of $\mathrm{IC}_{\zeta}$ generalizing [2]. As in [3], we show that Eis commutes with the Hecke functors with respect to the embedding $\check{M}_{n} \subset \check{G}_{n}$.

We formulate a conjectural functional equation of Eis. We also show as in [3] that the formation of Eisenstein series is transitive for the diagram $T \subset M \subset G$, where $T$ is a maximal torus of $M$.

In the case of $G=\mathrm{SL}_{2}$ we get some partial description of the Fourier coefficients of Eis, the answer is expressed in terms of a sheaf that appeared in the book [1] on factorizable sheaves (and also in [12, 22]). The relation so obtained between these Fourier coefficients and quantum groups seems a promising phenomenon that has to be better understood.

As an application, we get an important formula for the first Whittaker coefficient of our Eisenstein series for metaplectic extensions of $\mathrm{SL}_{2}$ (cf. Corollary 7.2). It turns out to be an $\ell$-adic analog of the space of conformal blocks in Wess-Zumino-Witten model studied in [1]. It also could be seen as a generalization of the notion of central value of an abelian L-function (cf. Remark 7.3).

Among other results, we construct new automorphic sheaves on $\widetilde{\operatorname{Bun}}_{G}$ in the case of $G=\mathrm{SL}_{2}$ and $X=\mathbb{P}^{1}$ corresponding to the trivial $\check{G}_{n}$-local system and a principal $\mathrm{SL}_{2} \rightarrow \check{G}_{n}$ of Arthur. We call them theta-sheaves as they generalize the theta-sheaves studied in [20].

\section{MAIN RESUltS}

2.0.3. Notations. Work over an algebraically closed field $k$. Let $G$ be a simple algebraic group over $k$. Assume it simply-connected (hopefully, the non simply-connected case could also be done using [26]). Let $T \subset B \subset G$ be a maximal torus and a Borel subgroup. Let $\mathfrak{g}$ 
be the Lie algebra of $G$. Write $\Lambda$ for the coweights lattice of $T, \check{\Lambda}$ for the weight lattice of $T$. Let $\Lambda^{+}$be the set of dominant coweights, $\breve{\Lambda}^{+}$the dominant weights. Write $\breve{h}$ for the dual Coxeter number of $G$. Write $W$ for the Weyl group of $(G, T)$, let $w_{0}$ be the longest element in $W$. Let $\mathcal{J}$ denote the set of vertices of the Dynkin diagram of $G$. For $i \in \mathcal{J}$ write $\alpha_{i}$ (resp., $\check{\alpha}_{i}$ ) for the simple coroot (resp., simple root) of $G$ corresponding to $i$.

We ignore the Tate twists everywhere (they are easy to recover if necessary).

Let $X$ be a smooth projective connected curve. Let Bun $_{G}$ be the stack of $G$-torsors on $X$. Let $F=k(X)$. For $x \in X$ we let $D_{x}$ denote the formal neighbourhood of $x$ in $X, D_{x}^{*}$ the punctured formal neighbourhood of $x \in X$. A trivial $G$-torsor on a base is denoted $\mathcal{F}_{G}^{0}$.

Let $\iota: \Lambda \otimes \Lambda \rightarrow \mathbb{Z}$ be the unique symmetric bilinear $W$-invariant form such that $\iota(\alpha, \alpha)=2$ for a short coroot $\alpha$. The induced map $\iota: \Lambda \rightarrow \check{\Lambda}$ is also denoted by $\iota$. If $\alpha$ is a simple coroot then $\iota(\alpha)=\frac{\iota(\alpha, \alpha)}{2} \check{\alpha}$. Our convention is that a super line is a $\mathbb{Z} / 2 \mathbb{Z}$-graded line.

Recall the groupoid $\mathcal{E}^{s}(T)$ defined in ([17], Section 3.2.1). Its objects are pairs: a symmetric bilinear form $\kappa: \Lambda \otimes \Lambda \rightarrow \mathbb{Z}$ and a central super extension $1 \rightarrow k^{*} \rightarrow \tilde{\Lambda}^{s} \rightarrow \Lambda \rightarrow 1$ such that its commutator is $\left(\gamma_{1}, \gamma_{2}\right)_{c}=(-1)^{\kappa\left(\gamma_{1}, \gamma_{2}\right)}$. This means that for every $\gamma \in \Lambda$ we are given a super line $\epsilon^{\gamma}$, and for $\gamma_{1}, \gamma_{2} \in \Lambda$ a $\mathbb{Z} / 2 \mathbb{Z}$-graded isomorphism

$$
c^{\gamma_{1}, \gamma_{2}}: \epsilon^{\gamma_{1}} \otimes \epsilon^{\gamma_{2}} \widetilde{\rightrightarrows} \epsilon^{\gamma_{1}+\gamma_{2}}
$$

such that $c$ is associative, and one has $c^{\gamma_{1}, \gamma_{2}}=(-1)^{\kappa\left(\gamma_{1}, \gamma_{2}\right)} c^{\gamma_{2}, \gamma_{1}} \sigma$. Here $\sigma: \epsilon^{\gamma_{1}} \otimes \epsilon^{\gamma_{2}} \widetilde{\rightrightarrows} \epsilon^{\gamma_{2}} \otimes \epsilon^{\gamma_{1}}$ is the super commutativity constraint. Then $\mathcal{E}^{s}(T)$ is a Picard groupoid with respect to the tensor product of central extensions.

We have a canonical object $\left(\iota, \tilde{\Lambda}^{\text {can }}\right) \in \mathcal{E}^{s}(T)$ corresponding to a canonical extension of $G$ by $K_{2}$ in the sense of ([5], Theorem 4.7). It is equipped with a $W$-equivariant structure. We pick once and for all a square root $\mathcal{E}_{X}$ of $\Omega$.

Recall the Picard groupoid $\mathcal{P}^{\theta}(X, \Lambda)$ of $\theta$-data from ([17], Section 4.2.1). Its objects are triples $\theta=(\kappa, \lambda, c)$, where $\kappa: \Lambda \otimes \Lambda \rightarrow \mathbb{Z}$ is a symmetric bilinear form, $\lambda$ is a rule that assigns to each $\gamma \in \Lambda$ a super line bundle $\lambda^{\gamma}$ on $X$, and $c$ is a rule that assigns to each pair $\gamma_{1}, \gamma_{2} \in \Lambda$ an isomorphism $c^{\gamma_{1}, \gamma_{2}}: \lambda^{\gamma_{1}} \otimes \lambda^{\gamma_{2}} \widetilde{\rightrightarrows} \lambda^{\gamma_{1}+\gamma_{2}} \otimes \Omega^{\kappa\left(\gamma_{1}, \gamma_{2}\right)}$ on $X$. They are subjects to the conditions from loc.cit. In particular, the parity of $\lambda^{\gamma}$ is $\kappa(\gamma, \gamma) \bmod 2$.

Denote by $\theta^{\text {can }} \in \mathcal{P}^{\theta}(X, \Lambda)$ the image of $\left(\iota, \tilde{\Lambda}^{\text {can }}\right)$ under the functor $\mathcal{E}^{s}(T) \rightarrow \mathcal{P}^{\theta}(X, \Lambda)$ of ([17], Lemma 4.1). That is, $\theta^{\text {can }}=\left(\iota, \lambda,{ }^{\prime} c\right)$, where $\lambda^{\gamma}=\mathcal{E}_{X}^{\otimes-\iota(\gamma, \gamma)} \otimes \epsilon^{\gamma}$, and

$$
{ }^{\prime} c^{\gamma_{1}, \gamma_{2}}: \lambda^{\gamma_{1}} \otimes \lambda^{\gamma_{2}} \widetilde{\rightrightarrows} \lambda^{\gamma_{1}+\gamma_{2}} \otimes \Omega^{\iota\left(\gamma_{1}, \gamma_{2}\right)}
$$

is the evident product obtained from (1).

For an algebraic stack $S$ locally of finite type write $\mathrm{D}(S)$ for the category introduced in ([16], Remark 3.21) and denoted $\mathrm{D}_{c}\left(S, \overline{\mathbb{Q}}_{\ell}\right)$ in loc.cit. It should be thought of as the unbounded derived category of constructible $\overline{\mathbb{Q}}_{\ell}$-sheaves on $S$.

If $V \rightarrow S$ and $V^{*} \rightarrow S$ are dual rank $r$ vector bundles on a base stack $S$, we normalize the Fourier transform $\operatorname{Four}_{\psi}: \mathrm{D}^{b}(V) \rightarrow \mathrm{D}^{b}\left(V^{*}\right)$ by $\operatorname{Four}_{\psi}(K)=\left(p_{V *}\right) !\left(\xi^{*} \mathcal{L}_{\psi} \otimes p_{V}^{*} K\right)[r]$, where $p_{V}, p_{V^{*}}$ are the projections, and $\xi: V \times V^{*} \rightarrow \mathbb{A}^{1}$ is the pairing. 
If $S$ is a stack, $L$ is a super line bundle on $S$ purely of parity zero, we will use the stack of $n$-th roots of $L$. Its $T$-point is a map $T \rightarrow S$, a super line bundle $\mathcal{U}$ on $T$ purely of parity

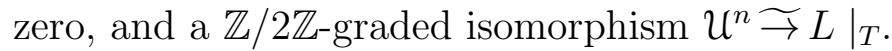

2.0.4. If $M \subset G$ is a Levi subgroup, denote by $\operatorname{Bun}_{M}$ the stack of $M$-torsors on $X$. For $\mu \in \pi_{1}(M)$, we denote by $\operatorname{Bun}_{M}^{\mu}$ the connected component of $\operatorname{Bun}_{M}$ classifying $M$-torsors on degree $-\mu$. This notation agrees with [3], but does not agree with [17]. Write $\operatorname{Pic}\left(\operatorname{Bun}_{T}\right)$ for the Picard groupoid of super line bundles on $\operatorname{Bun}_{T}$. If $\theta=(\kappa, \lambda, c)$ is an object of $\mathcal{P}^{\theta}(X, \Lambda)$ we also denote by $\lambda$ the super line bundle on $\operatorname{Bun}_{T}$ obtained from $\theta$ via the functor $\mathcal{P}^{\theta}(X, \Lambda) \rightarrow \operatorname{Pic}\left(\operatorname{Bun}_{T}\right)$ defined in ([17], Section 4.2.1, formula (18)).

The group $T$ acts on $\operatorname{Bun}_{T}$ by 2-automorphisms, so if $\mathcal{F} \in \operatorname{Bun}_{T}$ then $T$ acts naturally on the fibre at $\mathcal{F}$ of each line bundle on $\operatorname{Bun}_{T}$. According to our convention, for $\mathcal{F} \in \operatorname{Bun}_{T}^{\mu}$, $\mu \in \Lambda$ the group $T$ acts on $\lambda_{\mathcal{F}}$ by $-\kappa(\mu)$.

2.0.5. Let $\mathcal{L}$ be the line bundle on $\operatorname{Bun}_{G}$ with fibre $\operatorname{det} \mathrm{R} \Gamma\left(X, \mathfrak{g}_{\mathcal{F}}\right)^{-1} \otimes \operatorname{det} \mathrm{R} \Gamma(X, \mathfrak{g} \otimes \mathcal{O})$ at $\mathcal{F} \in \operatorname{Bun}_{G}$. This notation agrees with that of $[9$. Pick $n$ invertible in $k$. Pick a line bundle $\mathcal{L}_{c}$ on $\operatorname{Bun}_{G}$ equipped with $\mathcal{L}_{c}^{2 \check{h}} \widetilde{\Im} \mathcal{L}$, here $\mathcal{L}_{c}$ is a generator of $\operatorname{Pic}\left(\operatorname{Bun}_{G}\right) \widetilde{\rightarrow}$.

Let $\widetilde{\operatorname{Bun}}_{G, \mathcal{L}_{c}}$ be the stack of $n$-th roots of $\mathcal{L}_{c}$. Let $\bar{\zeta}: \mu_{n}(k) \rightarrow \overline{\mathbb{Q}}_{\ell}^{*}$ be an injective character. We are interested in the derived category $\mathrm{D}_{\bar{\zeta}}\left(\widetilde{\operatorname{Bun}}_{G, \mathcal{L}_{c}}\right)$ of $\overline{\mathbb{Q}}_{\ell}$-sheaves on $\widehat{\operatorname{Bun}}_{G, \mathcal{L}_{c}}$, on which $\mu_{n}(k)$ acts by $\bar{\zeta}$.

Assume that $N=2 \check{h} n$ is invertible in $k$. Write $\widetilde{\operatorname{Bun}}_{G}$ for the gerb of $N$-th roots of $\mathcal{L}$ over $\operatorname{Bun}_{G}$. Pick an injective character $\zeta: \mu_{N}(k) \rightarrow \overline{\mathbb{Q}}_{\ell}^{*}$ such that $\left.\zeta\right|_{\mu_{n}(k)}=\bar{\zeta}$. Denote by $\mathrm{D}_{\zeta}\left(\widetilde{\operatorname{Bun}}_{G}\right)$ the derived category of $\overline{\mathbb{Q}}_{\ell}$-sheaves on $\widetilde{\operatorname{Bun}}_{G}$ on which $\mu_{N}(k)$ acts by $\zeta$. We have a natural map $\alpha: \widetilde{\operatorname{Bun}}_{G, \mathcal{L}_{c}} \rightarrow \widetilde{\operatorname{Bun}}_{G}$, and $\alpha^{*}: \mathrm{D}_{\zeta}\left(\widetilde{\operatorname{Bun}}_{G}\right) \rightarrow \mathrm{D}_{\bar{\zeta}}\left(\widetilde{\operatorname{Bun}}_{G, \mathcal{L}_{c}}\right)$ is an equivalence.

Let $\check{G}_{n}$ be the $n$-th dual group of $G$ over $\overline{\mathbb{Q}}_{\ell}$ defined in ([9], Theorem 2.9). By construction, it is equipped with the Borel subgroup $\check{B}_{n}$ corresponding to $B \subset G$.

Let $\mathcal{L}_{T}$ be the restriction of $\mathcal{L}$ under the natural map $\operatorname{Bun}_{T} \rightarrow \operatorname{Bun}_{G}$. For $\check{\lambda} \in \check{\Lambda}$ and $\mathcal{F} \in \operatorname{Bun}_{T}$ denote by $\mathcal{L}_{\mathcal{F}}^{\check{\lambda}}$ the line bundle on $X$ obtained from $\mathcal{F}$ via the extensions of scalars $\check{\lambda}: T \rightarrow \mathbb{G}_{m}$. Given $\check{\lambda} \in \check{\Lambda}$ let $R^{\check{\lambda}}$ be the line bundle on $\operatorname{Bun}_{T}$ defined in ([17], 5.2.6, Example (2)). The fibre of $R^{\check{\lambda}}$ at $\mathcal{F} \in \operatorname{Bun}_{T}$ is

$$
\operatorname{det} \mathrm{R} \Gamma\left(X, \mathcal{L}_{\mathcal{F}}^{\check{\lambda}}\right) \otimes \operatorname{det} \mathrm{R} \Gamma\left(X, \mathcal{L}_{\mathcal{F}}^{-\check{\lambda}}\right) \otimes \operatorname{det} \mathrm{R} \Gamma(X, \mathcal{O})^{-2}
$$

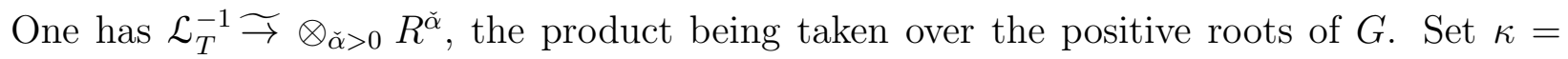
$-\sum_{\check{\alpha}>0} 2(\check{\alpha} \otimes \check{\alpha})$, the sum over the positive roots of $G$. This is a symmetric bilinear form $\kappa: \Lambda \otimes \Lambda \rightarrow \mathbb{Z}$. By $([9]$, Lemma 2.1), we have $\kappa=-2 \check{h} \iota$.

Define $\theta^{K i l}=(\kappa, \lambda, c)$ by $\theta^{K i l}=\left(\theta^{c a n}\right)^{-2 \breve{h}} \in \mathcal{P}^{\theta}(X, \Lambda)$, here Kil refers to the Killing form on $\Lambda$. The corresponding line bundle $\lambda$ on $\operatorname{Bun}_{T}$ identifies canonically with $\mathcal{L}_{T}$.

Let $\widetilde{\operatorname{Bun}}_{T}$ be the gerb of $N$-th roots of $\mathcal{L}_{T}$. Let $\mathrm{D}_{\zeta}\left(\widetilde{\mathrm{Bun}_{T}}\right)$ be the derived category of $\overline{\mathbb{Q}}_{\ell}$-sheaves on $\widetilde{\operatorname{Bun}}_{T}$ on which $\mu_{N}(k)$ acts by $\zeta$, similarly for $G$. Let

$$
\Lambda^{\sharp}=\{\mu \in \Lambda \mid \kappa(\mu, \nu) \in N \mathbb{Z} \text { for all } \nu \in \Lambda\}=\{\mu \in \Lambda \mid \iota(\mu, \nu) \in n \mathbb{Z} \text { for all } \nu \in \Lambda\}
$$


Set $T^{\sharp}=\mathbb{G}_{m} \otimes \Lambda^{\sharp}$.

In Section 3.1 we define a $\mu_{N} \times \mu_{N^{-}}$gerb $\overline{\operatorname{Bun}}_{\tilde{B}} \rightarrow \overline{\operatorname{Bun}}_{B}$ together with a daigram

$$
\widetilde{\operatorname{Bun}}_{T} \stackrel{\tilde{\mathfrak{q}}}{\leftarrow} \overline{\operatorname{Bun}}_{\tilde{B}} \stackrel{\tilde{\mathfrak{p}}}{\rightarrow} \widetilde{\operatorname{Bun}}_{G}
$$

We also define the perverse sheaf $\mathrm{IC}_{\zeta}$ on $\overline{\mathrm{Bun}}_{\tilde{B}}$. It gives rise to the Eisenstein series functor Eis : $\mathrm{D}_{\zeta}\left(\widetilde{\operatorname{Bun}}_{T}\right) \rightarrow \mathrm{D}_{\zeta}\left(\widetilde{\operatorname{Bun}}_{G}\right)$ given by

$$
\operatorname{Eis}(K)=\tilde{\mathfrak{p}}_{!}\left(\tilde{\mathfrak{q}}^{*} K \otimes \mathrm{IC}_{\zeta}\right)\left[-\operatorname{dim} \operatorname{Bun}_{T}\right]
$$

The analog of ([3], Theorem 2.1.2) in our setting is as follows.

Theorem 2.1. i) The functor $\mathrm{D}_{\zeta}\left(\widetilde{\operatorname{Bun}}_{T}\right) \rightarrow \mathrm{D}\left(\overline{\operatorname{Bun}}_{\tilde{B}}\right), K \mapsto \tilde{\mathfrak{q}}^{*} K \otimes \mathrm{IC}_{\zeta}\left[-\operatorname{dim} \operatorname{Bun}_{T}\right]$ is exact for the perverse t-structures and commutes with the Verdier duality.

ii) The functor Eis commutes with the Verdier duality.

Write $\check{T}^{\sharp}$ for the Langlands dual to $T^{\sharp}$ over $\overline{\mathbb{Q}}_{\ell}$. Recall that $\check{T}^{\sharp} \subset \check{B}_{n} \subset \check{G}_{n}$ is canonically included as a maximal torus. Set $\Lambda^{\sharp,+}=\Lambda^{\sharp} \cap \Lambda^{+}$, these are dominant weights of $\check{G}_{n}$. For $\nu \in \Lambda^{\sharp,+}$ denote by $V^{\nu}$ the irreducible representation of $\check{G}_{n}$ with highest weight $\nu$. For $\mu \in \Lambda^{\sharp}$ write $V^{\nu}(\mu) \subset V^{\nu}$ for the subspace on which $\check{T}^{\sharp}$ acts by $\mu$.

In Section 3.2 we define the action of the category of representations $\operatorname{Rep}\left(\check{G}_{n}\right)$ by the Hecke functors on $\mathrm{D}_{\zeta}\left(\widetilde{\operatorname{Bun}_{G}}\right)$. For $\nu \in \Lambda^{\sharp,+}$ we get the Hecke functor $\mathrm{H}_{G}^{\nu}: \mathrm{D}_{\zeta}\left(\widetilde{\operatorname{Bun}_{G}}\right) \rightarrow$ $\mathrm{D}_{\zeta}\left(\widetilde{\operatorname{Bun}}_{G} \times X\right)$.

The action of $\operatorname{Rep}\left(\check{T}^{\sharp}\right)$ on $\mathrm{D}_{\zeta}\left(\widetilde{\operatorname{Bun}}_{T}\right)$ by Hecke functors is defined in Section 3.2.1. For $\nu \in \Lambda^{\sharp}$ we get the Hecke functor $\mathrm{H}_{T}^{\nu}: \mathrm{D}_{\zeta}\left(\widetilde{\operatorname{Bun}_{T}}\right) \rightarrow \mathrm{D}_{\zeta}\left(\widetilde{\operatorname{Bun}_{T}} \times X\right)$. The following is an analog of ([3], Theorem 2.1.5) in our setting.

Theorem 2.2. For each $\nu \in \Lambda^{\sharp,+}$ and $K \in \mathrm{D}_{\zeta}\left(\widetilde{\operatorname{Bun}}_{T}\right)$ one has a functorial isomorphism

$$
\mathrm{H}_{G}^{\nu} \operatorname{Eis}(K) \widetilde{\rightrightarrows} \oplus_{\mu \in \Lambda^{\sharp}}(\operatorname{Eis} \otimes \mathrm{id}) \mathrm{H}_{T}^{\mu}(K) \otimes V^{\nu}(\mu),
$$

where Eis $\nabla \mathrm{id}: \mathrm{D}_{\zeta}\left(\widetilde{\operatorname{Bun}}_{T} \times X\right) \rightarrow \mathrm{D}_{\zeta}\left(\widetilde{\operatorname{Bun}}_{G} \times X\right)$ is the corresponding functor.

One checks in addition that the isomorphism of Theorem 2.2 is compatible with the convolution of Hecke functors.

Let $E$ be a $\check{T}^{\sharp}$-local system on $X$. For $\nu \in \Lambda^{\sharp}$ denote by $E^{\nu}$ the local system obtained from $E$ via the extension of scalars $\nu: \check{T}^{\sharp} \rightarrow \mathbb{G}_{m}$. Let $\mathcal{K}_{E}$ be the eigen-sheaf on $\widetilde{\operatorname{Bun}}_{T}$ constructed in ([17], Proposition 2.2). It satisfies the isomorphisms $\mathrm{H}_{T}^{\nu}\left(\mathcal{K}_{E}\right) \widetilde{\rightarrow} \mathcal{K}_{E} \otimes E^{-\nu}[1]$ for $\nu \in \Lambda^{\sharp}$. That is, $\mathcal{K}_{E}$ is a $E^{*}$-Hecke eigen-sheaf.

Corollary 2.1. Let $E_{\check{G}_{n}}$ be the $\check{G}_{n}$-local system induced from $E^{*}$. Then $\operatorname{Eis}\left(\mathcal{K}_{E}\right)$ is a $E_{\check{G}_{n}}{ }^{-}$ Hecke eigen-sheaf in $\mathrm{D}_{\zeta}\left({\widetilde{\operatorname{Bun}_{G}}}\right)$. 
2.0.6. Write $C^{*}\left(\check{G}_{n}\right)$ for the cocenter of $\check{G}_{n}$, the quotient of $\Lambda^{\sharp}$ by the roots lattice of $\check{G}_{n}$. The category $\operatorname{Rep}\left(\check{G}_{n}\right)$ is graded by $C^{*}\left(\check{G}_{n}\right)$ according to the action of the center of $\check{G}_{n}$. In Section 3.5 we introduce the corresponding grading on the derived category $\mathrm{D}_{\zeta}\left(\widetilde{\mathrm{Bun}_{G}}\right)$. We show in Proposition 3.6 that the Hecke functors for $G$ are compatible with these gradings on $\operatorname{Rep}\left(\check{G}_{n}\right)$ and on $\mathrm{D}_{\zeta}\left(\widetilde{\operatorname{Bun}}_{G}\right)$. This generalizes ([18], Lemma 1). We also describe the corresponding grading on the geometric Eisenstein series in Section 3.5 .

Write $\rho_{n}$ for the half sum of positive roots of $\breve{G}_{n}$. In Section 3.6 we define the twisted $W$ action on $\widetilde{\operatorname{Bun}}_{T}$ and formulate the conjectural functional equation for Eis (Conjecture 3.1). To this end, we also introduce the full triangluated subcategory $\mathrm{D}_{\zeta}\left(\widetilde{\operatorname{Bun}_{G}}\right)^{r e g} \subset \mathrm{D}_{\zeta}\left(\widetilde{\operatorname{Bun}_{G}}\right)$ of regular complexes. The appearance of the shift by $\rho_{n}$ here is analogous to the shift by $\rho$ in the functional equation for the usual geometric Eisenstein series ([3], Theorem 2.1.8). Our formulation of the functional equation is justified by the fact that it is compatible with our calculation of the constant terms of Eis for $G=\mathrm{SL}_{2}$ (see Proposition 7.3). Besides, it agrees with the results of [18]. In view of Theorem 2.5 below, the proof of the functional equation is reduced to the case of rank one. However, we don't know how to prove it for groups of rank one.

In Section 3.7 we give a relation between the action of $\operatorname{Bun}_{Z(G)}$ on $\widetilde{\operatorname{Bun}}_{G}$ and Hecke functors (and also the action of $\operatorname{Bun}_{Z(G)}$ on the Eisenstein series).

2.0.7. Parabolic Eisenstein series. Let $P \subset G$ be a parabolic containing $B, M$ be its Levi factor. Write $\mathcal{J}_{M} \subset \mathcal{J}$ for the corresponding subset. Write $\Lambda_{G, P}$ for the quotient of $\Lambda$ by the span on $\alpha_{i}, i \in \mathcal{J}_{M}$. Let $\mathcal{L}_{M}$ denote the restriction of $\mathcal{L}$ under $\operatorname{Bun}_{M} \rightarrow \operatorname{Bun}_{G}$. Let $\widetilde{\operatorname{Bun}}_{M}$ denote the gerb of $N$-th roots of $\mathcal{L}_{M}$.

In Section 4.1 we define a diagram of projections

$$
\widetilde{\operatorname{Bun}}_{M} \stackrel{\tilde{q}}{\leftarrow} \widetilde{\operatorname{Bun}}_{\tilde{P}} \stackrel{\tilde{\mathfrak{p}}}{\rightarrow} \widetilde{\operatorname{Bun}}_{G}
$$

and a perverse sheaf $\mathrm{IC}_{\zeta}$ on $\widetilde{\mathrm{Bun}}_{\tilde{P}}$ generalizing our previous definition for $B$. It gives rise to the parabolic Eisenstein series functor Eis : $\mathrm{D}_{\zeta}\left(\widetilde{\operatorname{Bun}}_{M}\right) \rightarrow \mathrm{D}_{\zeta}\left(\widetilde{\mathrm{Bun}}_{G}\right)$ given by

$$
\operatorname{Eis}(K)=\tilde{\mathfrak{p}}_{!}\left(\tilde{\mathfrak{q}}^{*} K \otimes \mathrm{IC}_{\zeta}\right)\left[-\operatorname{dim} \operatorname{Bun}_{M}\right]
$$

We write $\operatorname{Eis}_{M}^{G}=$ Eis if we need to express the dependence on $M$.

Theorem 2.3. i) The functor $\mathrm{D}_{\zeta}\left(\widetilde{\operatorname{Bun}_{M}}\right) \rightarrow \mathrm{D}\left(\widetilde{\operatorname{Bun}_{\tilde{P}}}\right), K \mapsto \tilde{\mathfrak{q}}^{*} K \otimes \mathrm{IC}_{\zeta}\left[-\operatorname{dim} \operatorname{Bun}_{M}\right]$ is exact for the perverse t-structures and commutes with the Verdier duality.

ii) The functor Eis : $\mathrm{D}_{\zeta}\left(\widetilde{\operatorname{Bun}}_{M}\right) \rightarrow \mathrm{D}_{\zeta}\left(\widetilde{\operatorname{Bun}}_{G}\right)$ commutes with the Verdier duality.

Remark 2.1. Let us explain at this point that the notations $\overline{\operatorname{Bun}}_{B}, \widetilde{\operatorname{Bun}}_{P}$ thoughout the paper are reserved for the corresponding Drinfeld compactifications (we assume $P \neq G$, so $\widetilde{\operatorname{Bun}}_{P}$ should not be confused with $\widetilde{\operatorname{Bun}}_{G}$ ). The gerbs over $\overline{\operatorname{Bun}}_{B}, \widetilde{\operatorname{Bun}}_{P}$ appearing in this paper such as $\overline{\operatorname{Bun}}_{\tilde{B}}, \widetilde{\operatorname{Bun}}_{\tilde{P}}\left(\right.$ or $\overline{\operatorname{Bun}}_{B, \tilde{G}}, \widehat{\operatorname{Bun}}_{P, \tilde{G}}$ below) are distinguished in our notation by some decoration above or next to the corresponding letter $B, P, G$. 
Set $\Lambda_{M, 0}=\left\{\lambda \in \Lambda \mid\left\langle\lambda \check{\alpha}_{i}\right\rangle=0\right.$ for all $\left.i \in \mathcal{J}_{M}\right\}$. Let $\check{\Lambda}_{M, 0}$ denote the dual lattice. In Section 4.1.1 we associate to $\kappa$ a homomoprhism $\kappa_{M}: \Lambda_{G, P} \rightarrow \check{\Lambda}_{M, 0}$ and prove the following generalization of ([17], Proposition 2.1).

Proposition 2.1. Let $\theta \in \Lambda_{G, P}$ with $\kappa_{M}(\theta) \notin N \check{\Lambda}_{M, 0}$. Then $\mathrm{D}_{\zeta}\left(\widetilde{\operatorname{Bun}_{M}}\right)$ vanishes.

Recall that $\mathcal{J}$ is canonically in bijection with the set of simple roots of $\check{G}_{n}$. Let $\check{M}_{n} \subset \check{G}_{n}$ be the standard Levi subgroup corresponding to $\mathcal{J}_{M}$.

In Section 4.2 we define the action of the category $\operatorname{Rep}\left(\check{M}_{n}\right)$ of $\check{M}_{n}$-representations on $\mathrm{D}_{\zeta}\left(\widetilde{\operatorname{Bun}}_{M}\right)$ by Hecke functors. Set $\Lambda_{M}^{\sharp,+}=\Lambda_{M}^{+} \cap \Lambda^{\sharp}$, these are dominant weights of $\check{M}_{n}$. For $\nu \in \Lambda_{M}^{\sharp,+}$ we get the Hecke functor $\mathrm{H}_{M}^{\nu}: \mathrm{D}_{\zeta}\left({\widetilde{\operatorname{Bun}_{M}}}\right) \rightarrow \mathrm{D}_{\zeta}\left(\widetilde{\operatorname{Bun}}_{M} \times X\right)$.

For $\nu \in \Lambda_{M}^{\sharp,+}$ denote by $U^{\nu}$ the irreducible representation of $\check{M}_{n}$ of highest weight $\nu$. The following is an analog of ([3], Theorem 2.3.7) in our setting.

Theorem 2.4. For $\lambda \in \Lambda^{\sharp,+}$ there is an isomorphism functorial in $K \in \mathrm{D}_{\zeta}\left(\widetilde{\operatorname{Bun}}_{M}\right)$

$$
\mathrm{H}_{G}^{\lambda} \operatorname{Eis}_{M}^{G}(K) \widetilde{\rightarrow} \underset{\nu \in \Lambda_{M}^{\sharp+}}{\oplus}\left(\operatorname{Eis}_{M}^{G} \otimes \mathrm{id}\right) \mathrm{H}_{M}^{\nu}(K) \otimes \operatorname{Hom}_{\check{M}_{n}}\left(U^{\nu}, V^{\lambda}\right),
$$

here $\operatorname{Eis}_{M}^{G} \otimes \mathrm{id}: \mathrm{D}_{\zeta}\left(\widetilde{\operatorname{Bun}_{M}} \times X\right) \rightarrow \mathrm{D}_{\zeta}\left(\widetilde{\operatorname{Bun}_{G}} \times X\right)$ is the corresponding functor.

One checks moreover that the isomorphism of Theorem 2.4 is compatible with the convolution of Hecke functors.

Corollary 2.2. Let $E$ be a $\check{M}_{n}$-local system on $X, \mathcal{K} \in \mathrm{D}_{\zeta}\left(\widetilde{\operatorname{Bun}}_{M}\right)$ be a E-Hecke eigen-sheaf. Then $\operatorname{Eis}_{M}^{G}(K) \in \mathrm{D}_{\zeta}\left({\widetilde{\operatorname{Bun}_{G}}}\right)$ is a $E_{\check{G}_{n}}$-Hecke eigen-sheaf. Here $E_{\check{G}_{n}}$ is the $\breve{G}_{n}$-local system induced from $E$.

One of our main results is Theorem 4.1 in Section 4.5 generalizing the description of the ICsheaf of $\widetilde{\operatorname{Bun}}_{P}$ from [2] to our twisted setting. Write $\Lambda_{G, P}^{\text {pos }}$ for the $\mathbb{Z}_{+}$-span of $\left\{\alpha_{i}, i \in \mathcal{J}_{-} \mathcal{J}_{M}\right\}$ in $\Lambda_{G, P}$. Pick $\theta \in \Lambda_{G, P}^{p o s}$. Let $\mathfrak{U}(\theta)$ be a decomposition of $\theta$ as in ([2], Section 1.4). Let $\check{\mathfrak{u}}_{n}(P)$ denote the Lie algebra of the unipotent radical of the standard parabolic $\check{P}_{n} \subset \check{G}_{n}$ corresponding to $\mathfrak{J}_{M} \subset \mathcal{J}$. One has a locally closed substack

$$
\mathfrak{u}(\theta) \widetilde{\operatorname{Bun}}_{P} \widetilde{\rightarrow} \operatorname{Bun}_{P} \times_{\mathrm{Bun}_{M}} \mathcal{H}_{M}^{+, \mathfrak{u}(\theta)} \hookrightarrow \widetilde{\mathrm{Bun}_{P}}
$$

(see Section 4.5 for these notations). Let $\mathfrak{u}(\theta)^{\operatorname{Bun}_{\tilde{P}}}$ be obtained from ${ }_{\mathfrak{u}(\theta)} \widetilde{\operatorname{Bun}}_{P}$ by the base change $\widetilde{\operatorname{Bun}}_{\tilde{P}} \rightarrow \widetilde{\operatorname{Bun}}_{P}$. Theorem 4.1 describes the $*$-restriction of $\mathrm{IC}_{\zeta}$ to $\mathfrak{u}(\theta) \widetilde{\operatorname{Bun}}_{\tilde{P}}$ in terms of the $\check{M}_{n}$-module $\check{\mathfrak{u}}_{n}(P)$ and the twisted Satake equivalence Loc : $\operatorname{Rep}\left(\check{M}_{n}\right) \widetilde{\rightarrow} \mathbb{P e r v}_{M, G, n}^{\natural}$ for $\check{M}_{n}$ (see Section 4.5). The proof actually establishes more (Theorem 5.2 and Corollary 5.1 do not reduce to Theorem 4.1).

In Section 6 we prove the following result, which is an analog of (3], Theorem 2.3.10) in our setting.

Theorem 2.5. There is an isomorphism of functors $\mathrm{D}_{\zeta}\left(\widetilde{\operatorname{Bun}_{T}}\right) \rightarrow \mathrm{D}_{\zeta}\left(\widetilde{\operatorname{Bun}_{G}}\right)$

$$
\operatorname{Eis}_{T}^{G} \simeq \operatorname{Eis}_{M}^{G} \circ \operatorname{Eis}_{T}^{M}
$$


2.0.8. In Section 7 we specialize to the case of $G=\mathrm{SL}_{2}$. As in Section 5.1, we have a $\mu_{N^{-}}$ gerb $\widetilde{Z}^{\theta} \rightarrow Z^{\theta}$ and a local version $\mathrm{IC}_{Z^{\theta}, \zeta}$ of the perverse sheaf $\mathrm{IC}_{\zeta}$. Here $\mathrm{IC}_{Z^{\theta}, \zeta}$ is a perverse sheaf on $\widetilde{Z}^{\theta}$ (see Sections 5.1 and 7 for notations). For $G=\mathrm{SL}_{2}$ the Zastava space $Z^{\theta}$ is a vector bundle over $X^{\theta}$, and it is important to calculate the Fourier transform $\operatorname{Four}_{\psi}\left(\mathrm{IC}_{Z^{\theta}, \zeta}\right)$ over the dual vector bundle. This calculation at the classical level is a part of the theory of Weyl group multiple Dirichlet series (see [4], 6] for a survey).

The description of $\mathrm{IC}_{Z^{\theta}, \zeta}$ is known (see Theorem 5.2 and Corollary 5.1). For $n=2$ the description of $\operatorname{Four}_{\psi}\left(\mathrm{IC}_{Z^{\theta}, \zeta}\right)$ is easily reduced to the description of $\mathrm{IC}_{Z^{\theta}, \zeta}$ itself (see Section 7.2.3, this was also used in [18]). For $n \geq 3$ we can not completely describe $\operatorname{Four}_{\psi}\left(\mathrm{IC}_{Z^{\theta}, \zeta}\right)$, and only establish Proposition 7.1, which calculates the desired Fourier transform over the open substack ${ }_{\Omega} \check{\tilde{Z}}_{\max }^{\theta} \subset{ }_{\Omega} \check{\tilde{Z}}^{\theta}$ (see Section 7.2 .3 for notations).

The answer in Proposition 7.1 is given in terms of the perverse sheaf $\mathrm{IC}_{\Omega} \tilde{X}^{\theta}, \bar{\zeta}$ that has been completely described in [1] in terms of cohomologies of a (part of) the quantum $\mathfrak{s l}_{2}$ at a suitable root of unity. This is a manifestation of the phenomenon that cohomology of quantum groups appear in the quantum geometric Langlands program (the quantum groups were brought into the quantum geometric Langlands program in [12, 22]).

In Proposition 7.2 we give a global application of Proposition 7.1, it expresses the nondegenerate Whittaker coefficients of $\operatorname{Eis}(K), K \in \mathrm{D}_{\zeta}\left(\widetilde{\operatorname{Bun}}_{T}\right)$ in terms of the perverse sheaf $\mathrm{IC}_{\check{Z}_{c}^{\theta}, \bar{\zeta}}$. This, in turn, yields a formula for the first Whittaker coefficient of $\operatorname{Eis}(K)$ (see Corollary 7.1 and 7.2). The complex appearing in Corollary 7.1 is an $\ell$-adic analog of the space of conformal blocks in Wess-Zumino-Witten model studied in [1].

In Section 7.3 we calculate the constant terms of $\operatorname{Eis}(K), K \in \mathrm{D}_{\zeta}\left(\widetilde{\operatorname{Bun}}_{T}\right)$ in terms of integral Hecke functors for $\widetilde{\mathrm{Bun}}_{T}$. Here 'integral' means that we apply Hecke functors at a collection of points and further integrate over this collection of points. The answer is given in Proposition 7.3, which (together with the results of [18]) explains our formulation of the functional equation.

2.0.9. Some special sheaves. Let $E$ be a $\check{T}^{\sharp}$-local system on $X, \mathcal{K}_{E} \in \mathrm{D}_{\zeta}\left(\widetilde{\mathrm{Bun}}_{T}\right)$ be the $E$-Hecke eigensheaf as in Corollary 2.1. This is a local system over the components of $\widetilde{\operatorname{Bun}}_{T}$ corresponding to $\Lambda^{\sharp}$. In Section 7.4 we describe some irreducible perverse sheaves $\mathrm{IC}(E, d) \in \mathrm{D}_{\zeta}\left(\widetilde{\operatorname{Bun}_{G}}\right), d>0$ that appear in $\operatorname{Eis}\left(\mathcal{K}_{E}\right)$.

We then specialize to the case of genus $g=0$. In this case $E$ is trivial, we set $\mathrm{IC}_{d}=$ $\operatorname{IC}\left(\overline{\mathbb{Q}}_{\ell}, d\right), d>0$ for brevity, and also define an irreducible perverse sheaf $\mathrm{IC}_{0}$ that appear in $\operatorname{Eis}\left(\mathcal{K}_{E}\right)$. Then any irreducible perverse sheaf appearing in $\operatorname{Eis}\left(\mathcal{K}_{E}\right)$ is isomorphic to some $\mathrm{IC}_{d}, d \geq 0$. Let $\mathcal{P}_{\zeta, n}$ denote the category of pure complexes on $\widetilde{\operatorname{Bun}}_{G}$, which are the direct sums of $\mathrm{IC}_{d}[r]\left(\frac{r}{2}\right), d \geq 0, r \in \mathbb{Z}$. Then $\mathcal{P}_{\zeta, n}$ is a module over $\operatorname{Rep}\left(\check{G}_{n}\right)$ acting by Hecke functors.

We explicitely describe the action of Hecke functors on each of the objects $\mathrm{IC}_{d}, d \geq 0$ (see Lemma 7.5 and Theorem 7.1). We also describe all the $*$-fibres of each of the perverse 
sheaf $\mathrm{IC}_{d}, d \geq 0$ (see Lemmas 7.3 and 7.4 ). Here is an immediate consequence of our results. Recall that $\check{G}_{n} \stackrel{\rightarrow}{\rightarrow} \mathrm{SL}_{2}$ for $n$ even (resp., $\check{G}_{n} \stackrel{\rightarrow}{\rightarrow} \mathrm{PSL}_{2}$ for $n$ odd).

Corollary 2.3. Let $m \geq 1$ with $n-m$ even. One has the equivalence $\mathcal{P}_{\zeta, n} \widetilde{\rightarrow}_{\zeta, m}$ sending $\mathrm{IC}_{d}[r]\left(\frac{r}{2}\right)$ to itself (and preserving direct sums). This equivalence commutes with the Hecke action (with respect to the evident isomorphism $\check{G}_{n} \widetilde{\rightarrow} \check{G}_{m}$ ).

Definition 2.1. For $n$ odd set Aut $=\mathrm{IC}_{0}$. For $n$ even set $\mathrm{Aut}=\mathrm{IC}_{0} \oplus \mathrm{IC}_{1}$. As in [20], we call Aut the theta-sheaf on $\widetilde{\operatorname{Bun}}_{G}$.

Corollary 2.4. The perverse sheaf Aut is a Hecke eigen-sheaf corresponding to the trivial $\check{G}_{n}$-local system and the principal $\mathrm{SL}_{2}$ of Arthur homomorphism $\mathrm{SL}_{2} \rightarrow \check{G}_{n}$.

2.0.10. Let us indicate some problems arising for future research:

1) Is it true that the theta-sheaves Aut satisfying the Hecke property as in Corollary 2.4 exist for all $G$ and any curve $X$ ?

2) Is it true that Aut constructed in Corollary 2.4 is the geometric analog of a matrix coefficient of a suitable nonramified automorphic representation of the corresponding $\mathbb{F}_{q}^{*} /\left(\mathbb{F}_{q}^{*}\right)^{n}$-metaplectic cover of $G$ ? Construct the corresponding representations for a local and global field (according to [15], they should exist).

3) Calculate the Fourier coefficients of the theta-sheaves given in Definition 2.1 .

2.0.11. In Appendix $\mathrm{A}$ we assume in addition that $k=\mathbb{F}_{q}$, and prove Theorem 2.6 below.

A version of our results holds also over $\mathbb{F}_{q}$, in particular the construction of $\mathcal{K}_{E}$ and the description of $\mathrm{IC}_{\zeta}$ given in Corollary 5.1. It is understood that in the corresponding description the Tate twists are recovered accordingly. Let $E$ be a $\check{T}^{\sharp}$-local system on $X, \mathcal{K}_{E}$ be the eigen-sheaf on $\widetilde{\operatorname{Bun}}_{T}$ constructed in ([17], Proposition 2.2). For $\mu \in \Lambda^{\sharp}$ write $\mathcal{K}_{E}^{\mu}$ for the restriction of $\mathcal{K}_{E}$ to $\widetilde{\operatorname{Bun}}_{T}^{\mu}$. By ([17], Proposition 2.1), $\mathcal{K}_{E}^{\mu}$ vanishes unless $\mu \in \Lambda^{\sharp}$.

For $\mu \in \Lambda^{\sharp}$ define $\operatorname{Eis}^{\prime}\left(\mathcal{K}_{E}^{\mu}\right)$ as

$$
\mathfrak{p}_{!}\left(\mathfrak{q}^{*}\left(\mathcal{K}_{E}^{\mu}\right) \otimes \mathrm{IC}_{\zeta}\right)
$$

where the maps $\mathfrak{p}, \mathfrak{q}$ are those of the diagram $\widetilde{\operatorname{Bun}}_{T} \stackrel{\mathfrak{q}}{\leftarrow} \operatorname{Bun}_{\tilde{B}} \stackrel{\mathfrak{p}}{\rightarrow} \widetilde{\operatorname{Bun}}_{G}$. Here $\operatorname{Bun}_{\tilde{B}}$ is obtained from $\operatorname{Bun}_{B}$ via the base change $\widetilde{\operatorname{Bun}}_{T} \times \widetilde{\operatorname{Bun}}_{G} \rightarrow \operatorname{Bun}_{T} \times \operatorname{Bun}_{G}$.

Denote by Funct $\left(\operatorname{Eis}\left(\mathcal{K}_{E}^{\mu}\right)\right)$ (resp., Funct $\left(\operatorname{Eis}^{\prime}\left(\mathcal{K}_{E}^{\mu}\right)\right)$ ) the function trace of Frobenius on the set $\widetilde{\operatorname{Bun}}_{T}\left(\mathbb{F}_{q}\right)$ corresponding to $\operatorname{Eis}\left(\mathcal{K}_{E}^{\mu}\right)$ and $\operatorname{Eis}^{\prime}\left(\mathcal{K}_{E}^{\mu}\right)$ respectively.

For $B=P$ the set $J$ defined in Section 4.5 identifies with the set of positive roots of $\check{G}_{n}$ for $\check{B}_{n}$. We denote by $\Lambda_{G, B}^{\text {pos,pos }}$ the free abelian semigroup with base $J$. Set $\Lambda^{\sharp, p o s}=\Lambda^{\sharp} \cap \Lambda^{\text {pos }}$. Let $\bar{c}_{P}: \Lambda_{G, B}^{\text {pos,pos }} \rightarrow \Lambda^{\sharp \text {,pos }}$ be the morphism of semigroups given on $J$ by the natural inclusion $J \hookrightarrow \Lambda^{\sharp, p o s}$. We write the elements of $\Lambda_{G, B}^{\text {pos,pos }}$ as $\mathfrak{B}(\theta)=\sum_{\nu \in J} n_{\nu} \nu$ with $\theta=\bar{c}_{P}(\mathfrak{B}(\theta))$.

The following is an analog of ([3], Theorem 2.2.11) in our setting. 
Theorem 2.6. The function Funct $\left(\operatorname{Eis}\left(\mathcal{K}_{E}^{\mu}\right)\right)$ vanishes unless $\mu \in \Lambda^{\sharp}$, and in the latter case it equals

$$
\sum_{\mathfrak{B}(\theta) \in \Lambda_{G, B}^{\text {pos,pos }}} \operatorname{Funct}\left(\operatorname{Eis}^{\prime}\left(\mathcal{K}_{E}^{\mu-\theta}\right)\right) \prod_{\nu \in J} \operatorname{Tr}\left(\operatorname{Fr}, \operatorname{R} \Gamma\left(X^{\left(n_{\nu}\right)} \otimes \overline{\mathbb{F}_{q}},\left(E^{-\nu}\right)^{\left(n_{\nu}\right)}\right) \otimes \overline{\mathbb{Q}}_{\ell}\left(n_{\nu}\right)\right),
$$

where $\mathfrak{B}(\theta)=\sum_{\nu \in J} n_{\nu} \nu$. Here $X^{(m)}$ denotes the $m$-th symmetric power of $X$, and for a local system $W$ on $X, W^{(m)}$ denotes its $m$-th symmetric power.

As in ([3], Theorem 2.2.12), Theorem 2.6 may be reformulated as follows in terms of generating series.

Consider the group ring $\overline{\mathbb{Q}}_{\ell}\left[\Lambda^{\sharp}\right]$, the ring of regular functions on the torus $\check{T}^{\sharp}$. For $\mu \in \Lambda^{\sharp}$ denote by $t^{\mu}$ the corresponding element of $\overline{\mathbb{Q}}_{\ell}\left[\Lambda^{\sharp}\right]$. Form a completed ring $\overline{\overline{\mathbb{Q}_{\ell}\left[\Lambda^{\sharp}\right.}}$ by allowing infinite expressions of the form

$$
\sum_{\mu} a_{\mu} t^{\mu}
$$

where $\mu$ runs over a subset of $\Lambda^{\sharp}$ of the form $\mu \geq \mu^{\prime}$, where $\mu^{\prime}$ is some fixed element of $\Lambda$.

The classical Eisenstein series can be thought of as a $\widetilde{\overline{\mathbb{Q}}_{\ell}\left[\Lambda^{\sharp}\right]}$-valued function on $\widetilde{\operatorname{Bun}_{G}}\left(\mathbb{F}_{q}\right)$ equal to

$$
\operatorname{Eis}_{c l}\left(\mathcal{K}_{E}\right)(t)=\sum_{\mu \in \Lambda^{\sharp}} \operatorname{Funct}\left(\operatorname{Eis}^{\prime}\left(\mathcal{K}_{E}^{\mu}\right)\right) t^{\mu} .
$$

Consider the modified Eisenstein series defined as

$$
\operatorname{Eis}_{\text {mod }}\left(\mathcal{K}_{E}\right)(t)=\sum_{\mu \in \Lambda^{\sharp}} \operatorname{Funct}\left(\operatorname{Eis}\left(\mathcal{K}_{E}^{\mu}\right)\right) t^{\mu},
$$

viewed as a function $\widetilde{\operatorname{Bun}_{G}}\left(\mathbb{F}_{q}\right) \rightarrow \widehat{\overline{\mathbb{Q}}_{\ell}\left[\Lambda^{\sharp}\right]}$. For $\nu \in J$ consider the abelian $L$-series $L\left(E^{*}, \nu, t\right) \in$ $\overline{\bar{Q}_{\ell}[\Lambda \sharp]}$ equal to

$$
\sum_{n \geq 0} \operatorname{Tr}\left(\mathrm{Fr}, \mathrm{R} \Gamma\left(X^{(n)} \otimes \overline{\mathbb{F}_{q}},\left(E^{-\nu}\right)^{(n)}\right) \otimes \overline{\mathbb{Q}}_{\ell}(n)\right) t^{n \nu}
$$

Theorem 2.7. For any $\check{T}^{\sharp}$-local system $E$ on $X$ one has

$$
\operatorname{Eis}_{\text {mod }}\left(\mathcal{K}_{E}\right)(t)=\operatorname{Eis}_{c l}\left(\mathcal{K}_{E}\right)(t) \prod_{\nu \in J} L\left(E^{*}, \nu, t\right)
$$

It is known that $\operatorname{Eis}_{c l}\left(\mathcal{K}_{E}\right)(t)$ satisfies the functional equation (cf. [23], [15]). This is a strong argument supporting our geometric functional equation (Conjecture 3.1).

\section{Principal GeOmetric Eisenstein SERIES}


3.1. Definitions. Keep notations of Section 2, Denote by $\overline{\mathrm{Bun}}_{B}$ the Drinfeld compactification of $\operatorname{Bun}_{B}$ from [3]. We have the diagram for the Drinfeld compactification $\operatorname{Bun}_{T} \stackrel{\bar{q}}{\leftarrow}$ $\overline{\operatorname{Bun}}_{B} \stackrel{\overline{\mathfrak{p}}}{\rightarrow} \operatorname{Bun}_{G}$. Write $\overline{\operatorname{Bun}}_{B, \tilde{G}}=\overline{\operatorname{Bun}}_{B} \times_{\operatorname{Bun}_{G}} \widetilde{\operatorname{Bun}}_{G}$. Set

$$
\overline{\operatorname{Bun}}_{\tilde{B}}=\overline{\operatorname{Bun}}_{B, \tilde{G}} \times_{\mathrm{Bun}_{T}} \widetilde{\operatorname{Bun}}_{T}
$$

A point of $\overline{\operatorname{Bun}}_{\tilde{B}}$ is given by $\mathcal{F}_{T} \in \mathrm{Bun}_{T}, \mathcal{F} \in \mathrm{Bun}_{G}$, a collection of inclusions

$$
\nu^{\check{\lambda}}: \mathcal{L}_{\mathcal{F}_{T}}^{\check{\lambda}} \hookrightarrow \mathcal{V}_{\mathcal{F}}^{\check{\lambda}}
$$

for all dominant weights $\check{\lambda}$ of $T$ satisfying the Plucker relations, and $\mathbb{Z} / 2 \mathbb{Z}$-graded lines of parity zero $\mathcal{U}, \mathcal{U}_{G}$ equipped with

$$
\mathcal{U}^{N} \widetilde{\rightarrow}\left(\mathcal{L}_{T}\right)_{\mathcal{F}_{T}}, \quad \mathcal{U}_{G}^{N} \widetilde{\rightarrow} \mathcal{L}_{\mathcal{F}}
$$

Here $\mathcal{V}^{\check{\lambda}}$ is the Weyl module corresponding to $\check{\lambda}$. Consider the open substack $\operatorname{Bun}_{B} \subset \overline{\operatorname{Bun}}_{B}$, let $\operatorname{Bun}_{\tilde{B}}$ be the restriction of $\overline{\operatorname{Bun}}_{\tilde{B}}$ to this open substack. For a point of $\operatorname{Bun}_{B}$ as above we have canonically $\mathcal{L}_{\mathcal{F}} \widetilde{\rightarrow}\left(\mathcal{L}_{T}\right)_{\mathcal{F}_{T}}$.

Let $a:$ Spec $k \rightarrow B\left(\mu_{N}\right)$ be the natural map, let $\mathcal{L}_{\zeta}$ be the direct summand in $a_{*} \overline{\mathbb{Q}}_{\ell}$ on which $\mu_{N}(k)$ acts by $\zeta$. Let

$$
\operatorname{Bun}_{B, \tilde{G}}=\operatorname{Bun}_{B} \times_{\mathrm{Bun}_{G}} \widetilde{\operatorname{Bun}_{G}},
$$

it classifies $\mathcal{F}_{B}, \mathcal{U}_{G}$ and an isomorphism $\mathcal{U}_{G}^{N} \widetilde{\rightarrow} \mathcal{L}_{\mathcal{F}_{B}}$. We get an isomorphism

$$
B\left(\mu_{N}\right) \times \operatorname{Bun}_{B, \tilde{G}} \widetilde{\rightarrow} \operatorname{Bun}_{\tilde{B}}
$$

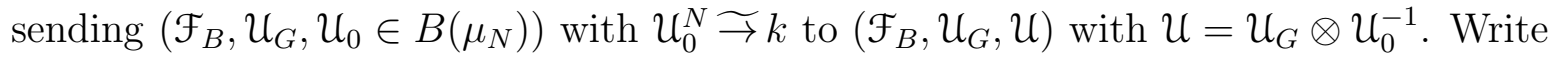

$$
\widetilde{\operatorname{Bun}}_{T} \stackrel{\tilde{q}}{\leftarrow} \overline{\operatorname{Bun}}_{\tilde{B}} \stackrel{\tilde{\mathfrak{p}}}{\rightarrow} \widetilde{\operatorname{Bun}}_{G}
$$

for the projections, so

$$
\tilde{\mathfrak{q}}\left(\mathcal{F}_{T}, \mathcal{F}, \nu, \mathcal{U}, \mathcal{U}_{G}\right)=\left(\mathcal{F}_{T}, \mathcal{U}\right) \text { and } \tilde{\mathfrak{p}}\left(\mathcal{F}_{T}, \mathcal{F}, \nu, \mathcal{U}, \mathcal{U}_{G}\right)=\left(\mathcal{F}, \mathcal{U}_{G}\right)
$$

View $\mathcal{L}_{\zeta} \otimes \mathrm{IC}\left(\operatorname{Bun}_{B, \tilde{G}}\right)$ as a perverse sheaf on $\operatorname{Bun}_{\tilde{B}}$ via (2). Let $\mathrm{IC}_{\zeta}$ be its intermediate extension to $\overline{\operatorname{Bun}}_{\tilde{B}}$.

Definition 3.1. For $K \in \mathrm{D}_{\zeta}\left(\widetilde{\operatorname{Bun}}_{T}\right)$ set

$$
\operatorname{Eis}(K)=\tilde{\mathfrak{p}}_{!}\left(\tilde{\mathfrak{q}}^{*} K \otimes \mathrm{IC}_{\zeta}\right)\left[-\operatorname{dim} \operatorname{Bun}_{T}\right]
$$

Let $\mu_{N}(k) \times \mu_{N}(k)$ act on $\overline{\operatorname{Bun}}_{\tilde{B}}$ by 2-automorphisms so that $\left(a, a_{G}\right)$ acts as $a$ on $\mathcal{U}$, as $a_{G}$ on $\mathcal{U}_{G}$ and trivially on $\left(\mathcal{F}_{T}, \mathcal{F}, \nu\right)$. Then $\left(a, a_{G}\right)$ acts on $\mathrm{IC}_{\zeta}$ by $\zeta\left(\frac{a_{G}}{a}\right)$. If $K \in \mathrm{D}_{\zeta}\left(\widetilde{\operatorname{Bun}_{T}}\right)$ then $a \in \mu_{N}(k) \subset \operatorname{Aut}(U)$ acts on $K$ as $\zeta(a)$. Then $\left(a, a_{G}\right)$ acts on $\tilde{\mathfrak{q}}^{*} K \otimes \mathrm{IC}_{\zeta}$ as $\zeta\left(a_{G}\right)$. So, $a_{G}$ acts on $\operatorname{Eis}(K)$ by $\zeta\left(a_{G}\right)$. 
3.2. Hecke functors. Let $\Lambda^{\sharp,+}=\Lambda^{\sharp} \cap \Lambda^{+}$. We use some notations from [9]. In particular, $\mathbf{O}=k[[t]] \subset \mathbf{F}=k((t)), \mathrm{Gr}_{G}=G(\mathbf{F}) / G(\mathbf{O})$. By abuse of notations, $\mathcal{L}$ will also denote the $\mathbb{Z} / 2 \mathbb{Z}$-graded line bundle on $\operatorname{Gr}_{G}$ whose fibre at $g G(\mathbf{O})$ is $\operatorname{det}\left(\mathfrak{g}(\mathbf{O}): \mathfrak{g}(\mathbf{O})^{g}\right)$. Write $\operatorname{Gra}_{G}$ for the punctured total space of $\mathcal{L}$. Let $\widetilde{\mathrm{Gr}}_{G}$ be the stack quotient of $\mathrm{Gra}_{G}$ by $\mathbb{G}_{m}$, where $z \in \mathbb{G}_{m}$ acts as the multiplication by $z^{N}$ with $N=2 \check{h} n$.

Let $\operatorname{Perv}_{G, n}$ be the category of $G(\mathbf{O})$-equivariant perverse sheaves on Gra $G$ with $\mathbb{G}_{m^{-}}$ monodromy $\zeta$. Let

$$
\operatorname{Perv}_{G, n}=\operatorname{Perv}_{G, n}[-1] \subset \mathrm{D}\left(\operatorname{Gra}_{G}\right)
$$

We view $\operatorname{Perv}_{G, n}$ as the category of $G(\mathbf{O})$-equivariant perverse sheaves on $\widetilde{\mathrm{Gr}}_{G}$, on which $\mu_{N}(k)$ acts by $\zeta$. Namely, a $G(\mathbf{O})$-equivariant perverse sheaf $K$ on $\widetilde{G r}_{G}$, on which $\mu_{N}(k)$ acts by $\zeta$, is identified with $\operatorname{pr}^{*} K \in \operatorname{Perv}_{G, n}$, where pr: $\operatorname{Gra}_{G} \rightarrow \widetilde{\operatorname{Gr}}_{G}$ is the quotient map under the $\mathbb{G}_{m}$-action.

As in ([9], Section 2.1), we pick a trivialization $\mathfrak{g}^{\check{\alpha}} \widetilde{\rightarrow} k$ of the root space for all the positive roots $\check{\alpha}$ and denote by $\Phi$ this collection of trivializations.

Let $\Omega(\mathbf{O})$ denote the completed module of relative differentials of $\mathbf{O}$ over $k$. Write $\Omega(\mathbf{O})^{\frac{1}{2}}$ for the groupoid of square roots of $\Omega(\mathbf{O})$. We pick $\mathcal{E} \in \Omega(\mathbf{O})^{\frac{1}{2}}$. As in ([9], Section 2.1), for $\nu \in \Lambda^{\sharp,+}$ we define the local system $E_{\mathcal{\varepsilon}}^{\nu}$ on $\operatorname{Gra}_{G}^{\nu}$ and $\mathcal{A}_{\varepsilon}^{\nu} \in \operatorname{Perv}_{G, n}$. By abuse of notation, $E_{\varepsilon}^{\nu}$ also denotes the corresponding local system on $\widetilde{\mathrm{Gr}_{G}}$.

Write $\mathcal{H}_{G}$ for the Hecke stack classifying $\mathcal{F}, \mathcal{F}^{\prime} \in \operatorname{Bun}_{G}, x \in X$ and an isomorphism $\beta:\left.\left.\mathcal{F}\right|_{X-x} \widetilde{\rightarrow} \mathcal{F}^{\prime}\right|_{X-x}$. We have a diagram

$$
\operatorname{Bun}_{G} \times X \stackrel{h \stackrel{\leftarrow}{\leftarrow} \times \pi}{\leftarrow} \mathcal{H}_{G} \stackrel{h_{G}}{\rightarrow} \operatorname{Bun}_{G},
$$

where $h_{G}^{\overleftarrow{G}}$ (resp., $h_{\vec{G}}$ ) sends the above point to $\mathcal{F}$ (resp., to $\left.\mathcal{F}^{\prime}\right)$. Here $\pi\left(\mathcal{F}, \mathcal{F}^{\prime}, \beta, x\right)=x$ These notations agree with 3 .

For $\nu \in \Lambda^{+}$we define $\overline{\mathcal{H}}_{G}^{\nu}$ as in ([3], Section 2.1.4). So, the closed substack $\overline{\mathcal{H}}_{G}^{\nu} \subset \mathcal{H}_{G}$ is given by the condition that for each $G$-module $\mathcal{V}$ whose weights are $\leq \check{\lambda}$ one has

$$
\nu_{\mathcal{F}}(-\langle\nu, \check{\lambda}\rangle x) \subset \mathcal{V}_{\mathcal{F}^{\prime}} \subset \mathcal{V}_{\mathcal{F}}\left(-\left\langle w_{0}(\nu), \check{\lambda}\right\rangle x\right)
$$

This is equivalent to requiring that $\mathcal{F}^{\prime}$ is in the position $\leq \nu$ with respect to $\mathcal{F}$ at $x$. Let $\mathcal{H}_{G}^{\nu} \subset \overline{\mathcal{H}}_{G}^{\nu}$ be the open substack given by the property that that $\mathcal{F}^{\prime}$ is in the position $\nu$ with respect to $\mathcal{F}$ at $x$.

Let $\operatorname{Gr}_{G, X}$ be the ind-scheme classifying $x \in X$, and a $G$-torsor $\mathcal{F}$ with a trivialization $\beta:\left.\mathcal{F} \rightarrow \mathcal{F}_{G}^{0}\right|_{X-x}$. Let $G_{X}$ be the functor classifying $x \in X$, and an automorphism of $\mathcal{F}_{G}^{0}$ over the formal neighbourhood of $x$. Write $\mathcal{L}_{X}$ for the $(\mathbb{Z} / 2 \mathbb{Z}$-graded of parity zero) line bundle on $\operatorname{Gr}_{G, X}$ whose fibre at $(\mathcal{F}, x, \beta)$ is $\operatorname{det} \operatorname{R} \Gamma\left(X, \mathfrak{g} \otimes \mathcal{O}_{X}\right) \otimes \operatorname{det} \operatorname{R} \Gamma\left(X, \mathfrak{g}_{\mathcal{F}}\right)^{-1}$. Let $\widetilde{\operatorname{Gr}}_{G, X}$ be the gerb of $N$-th roots of $\mathcal{L}_{X}$ over $\operatorname{Gr}_{G, X}$. Let $\operatorname{Gra}_{G, X}$ be the punctured total space of the line bundle $\mathcal{L}_{X}$ on $\operatorname{Gr}_{G, X}$.

Write $\operatorname{Bun}_{G, X}$ for the stack classifying $\left(\mathcal{F} \in \operatorname{Bun}_{G}, x \in X, \nu\right)$, where $\nu:\left.\mathcal{F} \widetilde{\rightarrow} \mathcal{F}_{G}^{0}\right|_{D_{x}}$ is a trivialization over the formal neighbourhood $D_{x}$ of $x$. Note that $\operatorname{Bun}_{G, X}$ is a $G_{X}$-torsor over $\operatorname{Bun}_{G} \times X$. Set $\widetilde{\operatorname{Bun}}_{G, X}=\widetilde{\operatorname{Bun}}_{G} \times_{\operatorname{Bun}_{G}} \operatorname{Bun}_{G, X}$. 
Let $\gamma^{\leftarrow}$ (resp., $\gamma^{\rightarrow}$ ) denote the isomorphism $\operatorname{Bun}_{G, X} \times_{G_{X}} \operatorname{Gr}_{G, X} \widetilde{\rightarrow} \mathcal{H}_{G}$ such that the projection to the first term corresponds to $h_{G}^{\leftarrow}$ (resp., $h_{G}$ ). The line bundle $\mathcal{L} \otimes \mathcal{L}_{X}$ on $\operatorname{Bun}_{G, X} \times \operatorname{Gr}_{G, X}$ is naturally $G_{X}$-equivariant, we denote by $\mathcal{L} \tilde{\otimes} \mathcal{L}_{X}$ its descent to

$$
\operatorname{Bun}_{G, X} \times_{G_{X}} \operatorname{Gr}_{G, X}
$$

Note that $\overline{\mathcal{H}}_{G}^{\nu}$ identifies with $\operatorname{Bun}_{G, X} \times_{G_{X}} \overline{\mathrm{Gr}}_{G, X}^{\nu}$ under $\gamma^{\leftarrow}$. We have canonically

$$
\left(\gamma^{\rightarrow}\right)^{*}\left(h_{G}^{\overleftarrow{ }}\right)^{*} \mathcal{L} \Im \mathcal{L} \tilde{\otimes} \mathcal{L}_{X}
$$

Let $\mathcal{H}_{\tilde{G}}$ be the stack obtained from $\widetilde{\operatorname{Bun}}_{G} \times \widetilde{\operatorname{Bun}}_{G}$ be the base change $h^{\leftarrow} \times h^{\rightarrow}: \mathcal{H}_{G} \rightarrow$ $\operatorname{Bun}_{G} \times \operatorname{Bun}_{G}$. Denote by $\tilde{h}_{G}^{\leftarrow}, \tilde{h}_{G}$ the projections in the diagram

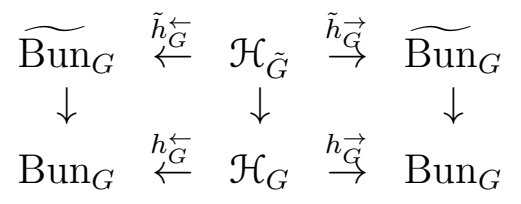

The stack $\mathcal{H}_{\tilde{G}}$ classifies $\left(\mathcal{F}, \mathcal{F}^{\prime}, \beta, x\right) \in \mathcal{H}_{G}$ and lines $\mathcal{U}, \mathcal{U}^{\prime}$ equipped with $\mathcal{U}^{N} \Im \mathcal{L}_{\mathcal{F}}, \mathcal{U}^{\prime N} \widetilde{\mathcal{L}_{\mathcal{F}^{\prime}}}$.

The isomorphism (4) yields a $G_{X}$-torsor $\tilde{\gamma} \rightarrow: \widetilde{\operatorname{Bun}}_{G, X} \times_{X} \widetilde{\operatorname{Gr}}_{G, X} \rightarrow \mathcal{H}_{\tilde{G}}$ extending the $G_{X}$-torsor

$$
\operatorname{Bun}_{G, X} \times_{X} \operatorname{Gr}_{G, X} \rightarrow \operatorname{Bun}_{G, X} \times_{G_{X}} \operatorname{Gr}_{G, X} \stackrel{\gamma \rightarrow}{\rightarrow} \mathcal{H}_{G}
$$

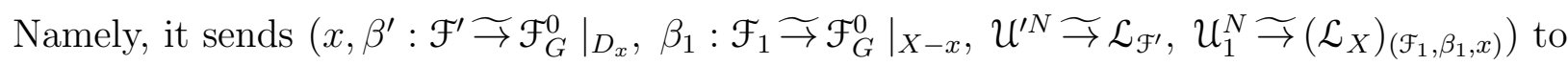

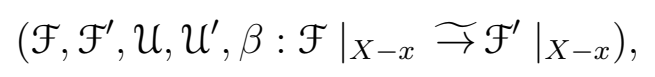

where $\mathcal{F}$ is obtained as the gluing of $\left.\mathcal{F}^{\prime}\right|_{X-x}$ with $\left.\mathcal{F}_{1}\right|_{D_{x}}$ via $\beta_{1}^{-1} \circ \beta^{\prime}:\left.\mathcal{F}^{\prime} \widetilde{\mathcal{F}_{1}}\right|_{D_{x}^{*}}$. We have canonically $\mathcal{L}_{\mathcal{F}^{\prime}} \otimes\left(\mathcal{L}_{X}\right)_{\left(\mathcal{F}_{1}, \beta_{1}, x\right)} \underset{\rightarrow}{\mathcal{L}} \mathcal{L}_{\mathcal{F}}$, and $\mathcal{U}=\mathcal{U}^{\prime} \otimes \mathcal{U}_{1}$ is equipped with the induced isomorphism $\mathfrak{U}^{N} \widetilde{\sim} \mathcal{L}_{\mathcal{F}}$.

Let $\operatorname{Sph}\left(\widetilde{G r}_{G, X}\right)$ be the category of $G_{X}$-equivariant perverse sheaves on $\widetilde{G r}_{G, X}$. Now for $\mathcal{S} \in \operatorname{Sph}\left(\widetilde{\operatorname{Gr}}_{G, X}\right)$ and $\mathcal{T} \in \mathrm{D}\left(\widetilde{\operatorname{Bun}}_{G}\right)$ we can form their twisted tensor product $(\mathcal{T} \tilde{\otimes} \mathcal{S})^{r}$, which is the descent via $\tilde{\gamma} \rightarrow$. Similarly, one may define $\tilde{\gamma}^{\leftarrow}$ and the complex $(\mathcal{T} \tilde{\otimes} \mathcal{S})^{l}$ on $\mathcal{H}_{\tilde{G}}$ (as in [3], Section 3.2.4).

We also denote the composition $\mathcal{H}_{\tilde{G}} \rightarrow \mathcal{H}_{G} \stackrel{\pi}{\rightarrow} X$ by $\pi$. Let $\mathcal{S} \in \operatorname{Sph}\left(\widetilde{\operatorname{Gr}}_{G, X}\right)$ and $\mathcal{T} \in$ $\mathrm{D}_{\zeta}\left(\widetilde{\operatorname{Bun}}_{G}\right)$. If $a_{1} \in \mu_{N}(k) \subset \operatorname{Aut}\left(\mathcal{U}_{1}\right)$ acts on $\mathcal{S}$ as $\zeta\left(a_{1}\right)$ then $\left(a, a^{\prime}\right) \in \mu_{N}(k) \times \mu_{N}(k) \subset$ $\operatorname{Aut}(\mathcal{U}) \times \operatorname{Aut}\left(\mathcal{U}^{\prime}\right)$ acts on $(\mathcal{T} \tilde{\otimes} \mathcal{S})^{r}$ as $\zeta(a)$, so $\left(\tilde{h}_{G} \overleftarrow{ } \times \pi\right)_{!}\left((\mathcal{T} \tilde{\nabla} \mathcal{S})^{r}\right) \in \mathrm{D}_{\zeta}\left(\widetilde{\operatorname{Bun}_{G}} \times X\right)$.

As in [9] write $\operatorname{Perv}_{G, n, X}$ for the category of compexes $K \in \mathrm{D}\left(\widetilde{G r}_{G, X}\right)$ such that $K[1]$ is perverse, $G_{X}$-equivariant, and $\mu_{N}(k)$ acts on $K$ by $\zeta$. Our choice of $\mathcal{E}_{X}$ (see Section 2.0.3) yields a fully faithful localization functor

$$
\tau^{0}: \operatorname{Perv}_{G, n} \rightarrow \operatorname{Perv}_{G, n, X}
$$

defined in ([9], Section 2.3). Now for $\nu \in \Lambda^{\sharp,+}$ we get $\mathcal{A}^{\nu}:=\tau^{0}\left(\mathcal{A}_{\varepsilon}^{\nu}\right) \in \operatorname{Perv}_{G, n, X}$. Define

$$
\mathrm{H}_{G}^{\nu}: \mathrm{D}_{\zeta}\left(\widetilde{\operatorname{Bun}_{G}}\right) \rightarrow \mathrm{D}_{\zeta}\left(\widetilde{\operatorname{Bun}_{G}} \times X\right)
$$


by

$$
\mathrm{H}_{G}^{\nu}(\mathcal{T})=\left(\tilde{h}_{G}^{\overleftarrow{ }} \times \pi\right) !\left(\left(\mathcal{T} \tilde{\otimes} \mathcal{A}^{-w_{0}(\nu)}\right)^{r}\right)
$$

We used that $\Lambda^{\sharp,+}$ is preserved by $-w_{0}$. This definition agrees with [3]. For $\nu \in \Lambda^{\sharp,+}$ write also $\mathrm{IC}^{\nu}=\left(\mathrm{IC}\left(\widetilde{\operatorname{Bun}}_{G, X}\right) \tilde{\otimes} \mathcal{A}^{-w_{0}(\nu)}\right)^{r}$. Let

$$
\overline{\mathcal{H}}_{\tilde{G}}^{\nu}=\mathcal{H}_{\tilde{G}} \times_{\mathcal{H}_{G}} \overline{\mathcal{H}}_{G}^{\nu}
$$

Then $\mathrm{IC}^{\nu}$ is an irreducible perverse sheaf, the extension by zero from $\overline{\mathcal{H}}_{\tilde{G}}^{\nu}$. For $\mathcal{T} \in \mathrm{D}_{\zeta}\left(\widetilde{\operatorname{Bun}}_{G}\right)$ we may rewrite

$$
\left.\mathrm{H}_{G}^{\nu}(\mathcal{T})=\left(\tilde{h}_{G}^{\overleftarrow{ }} \times \pi\right)_{!}\left(\left(\tilde{h}_{G}\right)^{*} \mathcal{T} \otimes \mathrm{IC}^{\nu}\right)\right)\left[-\operatorname{dim} \operatorname{Bun}_{G}\right]
$$

Recall the covariant functor $\star: \mathbb{P e r v}_{G, n, \zeta^{-1}} \rightarrow \operatorname{Perv}_{G, n, \zeta}$ defined in ([9], Remark 2.8), see also ([21], Remark 2.2). For $\nu \in \Lambda^{\sharp,+}$ it sends $\mathcal{A}_{\varepsilon}^{\nu}$ to $\mathcal{A}_{\varepsilon}^{-w_{0}(\nu)}$. More generally, for $\mathcal{S} \in \operatorname{Perv}_{G, n}, \mathcal{T} \in \mathrm{D}_{\zeta}\left(\widetilde{\operatorname{Bun}}_{G}\right)$ set

$$
\mathrm{H}_{G}^{\leftarrow}(\mathcal{S}, \mathcal{T})=\left(\tilde{h}_{G}^{\leftarrow} \times \pi\right) !\left(\left(\mathcal{T} \tilde{\otimes} \tau^{0}(\star \mathcal{S})\right)^{r}\right) \quad \text { and } \quad \mathrm{H}_{G}(\mathcal{S}, \mathcal{T})=\left(\tilde{h}_{G} \times \pi\right) !\left(\mathcal{T} \tilde{\otimes} \tau^{*}(\mathcal{S})\right)^{l}
$$

These are analogs of the corresponding functors from ([3], Section 3.2.4), they satisfy similar properties. In particular, $\mathrm{H}_{G}^{\leftarrow}$ (resp., $\left.\mathrm{H}_{G}\right)$ defines a left (resp., right) action on $\mathrm{D}_{\zeta}\left(\widetilde{\operatorname{Bun}_{G}}\right)$.

3.2.1. Hecke functors for $T$. For $\nu \in \Lambda^{\sharp}$ define the Hecke functor

$$
\mathrm{H}_{T}^{\nu}: \mathrm{D}_{\zeta}\left(\widetilde{\operatorname{Bun}}_{T}\right) \rightarrow \mathrm{D}_{\zeta}\left(\widetilde{\operatorname{Bun}}_{T} \times X\right)
$$

as follows. Our definition will be consistent with (5) but will differ from those of [17].

Recall $\theta^{K i l}=(\kappa, \lambda, c)$ from Section 2.0.5. For $\nu \in \Lambda$ the line bundle $\lambda^{\nu}$ is the restriction of $\mathcal{L}_{T}$ under $X \rightarrow \operatorname{Bun}_{T}, x \mapsto \mathcal{F}_{T}^{0}(\nu x)$. Note that $\lambda^{\nu} \Im \Omega^{\breve{h} \iota(\nu, \nu)}$ by ([17], Lemma 4.1).

Let $\left(\iota, \tilde{\Lambda}^{\sharp \text {,can }}\right)$ denote the restriction of $\left(\iota, \tilde{\Lambda}^{\text {can }}\right)$ to $\Lambda^{\sharp}$, its is equipped with a $W$-equivariant structure. We pick an object $\left(\frac{\iota}{n}, \tilde{\Lambda}^{\sharp}\right)$ in $\mathcal{E}^{s}\left(T^{\sharp}\right)$ and a $W$-equivariant isomorphism

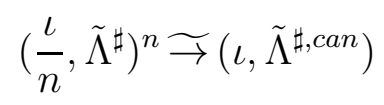

in $\mathcal{E}^{s}\left(T^{\sharp}\right)$. Now using ([17], Lemma 4.1), the above object yields $\left(\tau, \frac{\kappa}{N}, c\right) \in \mathcal{P}^{\theta}\left(X, \Lambda^{\sharp}\right)$ and a $W$-equivariant isomorphism

$$
\left.\left(\tau, \frac{\kappa}{N}, c\right)^{N} \underset{\rightarrow}{\rightarrow} \theta^{K i l}\right|_{\Lambda^{\sharp}}
$$

Note that $\frac{\kappa}{N}=-\frac{\iota}{n}: \Lambda^{\sharp} \otimes \Lambda^{\sharp} \rightarrow \mathbb{Z}$ may take odd values, so $\tau$ is a super line bundle in general.

We also write $\tau$ for the line bundle on $\operatorname{Bun}_{T^{\sharp}}$ obtained from (7) applying the corresponding functor $\mathcal{P}^{\theta}\left(X, \Lambda^{\sharp}\right) \rightarrow \mathcal{P}$ ic $\left(\operatorname{Bun}_{T^{\sharp}}\right)$ as in ([17], Section 4.2.1). It is equipped with a $W$ equivariant structure and a $W$-equivariant isomorphism $\tau^{N} \widetilde{\rightarrow} i_{X}^{*} \lambda$. Here $i_{X}: \operatorname{Bun}_{T^{\sharp}} \rightarrow \operatorname{Bun}_{T}$ is the natural map. 
For $\nu \in \Lambda^{\sharp}$ let $m^{\nu}: \widetilde{\operatorname{Bun}}_{T} \times X \rightarrow \widetilde{\operatorname{Bun}}_{T}$ be the map sending $x \in X,(\mathcal{F}, \mathcal{U}) \in \widetilde{\operatorname{Bun}}_{T}$ to $\left(\mathcal{F}^{\prime}=\mathcal{F}(-\nu x), \mathcal{U}^{\prime}\right)$, where

$$
\mathcal{U}^{\prime}=\mathcal{U} \otimes\left(\mathcal{L}_{\mathcal{F}}^{-\frac{\kappa(\nu)}{N}}\right)_{x} \otimes \tau_{\mathcal{O}(-\nu x)}
$$

equipped with the induced isomorphism $\mathcal{U}^{\prime N} \widetilde{\rightarrow}\left(\mathcal{L}_{T}\right)_{\mathcal{F}^{\prime}}$. It coincides with the map denoted $m_{-\nu}$ in ([17], Section 5.2.3). The Hecke functor

$$
\mathrm{H}_{T}^{\nu}: \mathrm{D}_{\zeta}\left(\widetilde{\operatorname{Bun}_{T}}\right) \rightarrow \mathrm{D}_{\zeta}\left(\widetilde{\operatorname{Bun}_{T}} \times X\right)
$$

is defined by $\mathrm{H}_{T}^{\nu}(K)=\left(m^{\nu}\right)^{*} K[1]$.

3.3. Proof of Theorem 2.2. We will use the following result. Let $\mathrm{Gr}_{T}, \mathrm{Gr}_{B}$ be the affine grassmanians for $T, B$. Let $\widetilde{\mathrm{Gr}}_{T} \rightarrow \widetilde{\mathrm{Gr}}_{B} \rightarrow \widetilde{\mathrm{Gr}}_{G}$ be obtained from $\mathrm{Gr}_{T} \rightarrow \mathrm{Gr}_{B} \rightarrow \mathrm{Gr}_{G}$ by the base change $\widetilde{G r}_{G} \rightarrow \operatorname{Gr}_{G}$. As in ([9], Section 4.1) for $\nu \in \Lambda^{\sharp,+}$ and $\mu \in \Lambda$ one has the diagram of ind-stacks

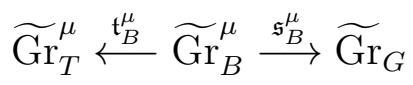

The connected component $\widetilde{\mathrm{Gr}}_{T}^{\mu}$ is the one containing $t^{\mu} T(\mathbf{O})$, similarly for $\widetilde{\mathrm{Gr}}_{B}^{\mu}$.

If $\mu \in \Lambda^{\sharp}$ as in ([9], Section 4.2) we denote by $a_{\mu}: \mathcal{E}_{\bar{c}}^{\iota(\mu, \mu) / n}-\{0\} \rightarrow \Omega_{\bar{c}}^{\check{h}_{\iota}(\mu, \mu)}-\{0\}$ the map $z \mapsto z^{2 \check{h} n}$.

Proposition 3.1 (9]). Let $\nu \in \Lambda^{\sharp,+}, \mu \in \Lambda$. The complex $a_{\mu}^{*}\left(\mathfrak{t}_{B}^{\mu}\right) !\left(\mathfrak{s}_{B}^{\mu}\right)^{*} \mathcal{A}_{\varepsilon}^{\nu}$ vanishes unless $\mu \in \Lambda^{\sharp}$. In the latter case this complex is constant and identifies canonically with $V^{\nu}(\mu)[-\langle\mu, 2 \check{\rho}\rangle]$.

3.3.1. Pick $\nu \in \Lambda^{\sharp,+}$. Consider a version of the basic diagram from ([3], Section 3.1.1). Set $\bar{Z}=\overline{\mathcal{H}}_{\tilde{G}}^{\nu} \times_{\operatorname{Bun}_{G}} \overline{\operatorname{Bun}}_{\tilde{B}}$, where we used the map $\tilde{h}_{G}: \overline{\mathcal{H}}_{\tilde{G}}^{\nu} \rightarrow \widetilde{\operatorname{Bun}}_{G}$ to define the fibred product.

Lemma 3.1. There is a morphism of stacks $\phi: \bar{Z} \rightarrow \overline{\operatorname{Bun}}_{\tilde{B}} \times X$ that fits into a commutative diagram

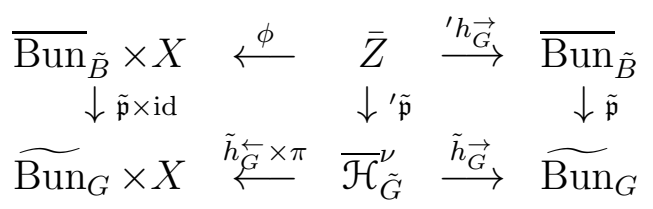

Proof A point of $\bar{Z}$ is given by

$$
\left(\mathcal{F}_{T}^{\prime}, \mathcal{F}^{\prime}, \nu^{\prime}, \mathcal{U}^{\prime N} \widetilde{\rightarrow}\left(\mathcal{L}_{T}\right)_{\mathcal{F}_{T}^{\prime}}, \mathcal{U}_{G}^{\prime N} \widetilde{\rightarrow} \mathcal{L}_{\mathcal{F}^{\prime}}\right) \in \overline{\mathrm{Bun}}_{\tilde{B}}
$$

and $\left(x, \mathcal{F}, \mathcal{F}^{\prime}, \beta, \mathcal{U}_{G}^{\prime N} \Im \mathcal{L}_{\mathcal{F}^{\prime}}, \mathcal{U}_{G}^{N} \Im \mathcal{L}_{\mathcal{F}}\right) \in \overline{\mathcal{H}}_{\tilde{G}}^{\nu}$. For this point we let $\mathcal{F}_{T}=\mathcal{F}_{T}^{\prime}\left(w_{0}(\nu) x\right)$ with the system of induced inclusions

$$
\nu^{\check{\lambda}}: \mathcal{L}_{\mathcal{F}_{T}^{\prime}}^{\check{\lambda}}\left(\left\langle w_{0}(\nu), \check{\lambda}\right\rangle x\right) \hookrightarrow \mathcal{V}_{\mathcal{F}_{G}}^{\check{\lambda}}
$$

for all $\check{\lambda} \in \check{\Lambda}^{+}$. The map $\phi$ sends the above point to

$$
\left(\mathcal{F}_{T}, \mathcal{F}, \nu, \mathcal{U}_{G}^{N} \widetilde{\rightarrow} \mathcal{L}_{\mathcal{F}}, \mathcal{U}^{N} \Im\left(\mathcal{L}_{T}\right)_{\mathcal{F}_{T}}\right),
$$


where $\left(\mathcal{F}_{T}, \mathcal{U}, \mathcal{U}^{N} \widetilde{\rightarrow}\left(\mathcal{L}_{T}\right)_{\mathcal{F}_{T}}\right)$ is the image of $\left(\mathcal{F}_{T}^{\prime}, \mathcal{U}^{\prime N} \widetilde{\Im}\left(\mathcal{L}_{T}\right)_{\mathcal{F}_{T}^{\prime}}, x\right)$ under $m^{-w_{0}(\nu)}$.

Set

$$
\mathrm{IC}(\bar{Z})_{\zeta}=\left({ }^{\prime} \tilde{\mathfrak{p}}\right)^{*} \mathrm{IC}^{\nu} \otimes\left({ }^{\prime} h_{G}\right)^{*} \mathrm{IC}_{\zeta}\left[-\operatorname{dim} \operatorname{Bun}_{G}\right]
$$

Since $\tilde{h}_{G}$ in (9) is a locally trivial fibration in smooth topology, $\operatorname{IC}(\bar{Z})_{\zeta}$ is an irreducible perverse sheaf on $\bar{Z}$. For a point (10) let

$$
\left(a, a^{\prime}, b^{\prime}\right) \in \mu_{N}(k) \times \mu_{N}(k) \times \mu_{N}(k) \subset \operatorname{Aut}\left(\mathcal{U}_{G}\right) \times \operatorname{Aut}\left(\mathcal{U}_{G}^{\prime}\right) \times \operatorname{Aut}\left(\mathcal{U}^{\prime}\right)
$$

acting trivially on $\left(\mathcal{F}_{T}^{\prime}, \mathcal{F}^{\prime}, \mathcal{F}\right)$. This 2-automorphism acts on $\operatorname{IC}(\bar{Z})_{\zeta}$ as $\zeta\left(\frac{a}{b^{\prime}}\right)$.

For each $\mu \in \Lambda_{G}^{\text {pos }}$ one has the closed embedding $i_{\mu}: \overline{\operatorname{Bun}}_{B} \times X \hookrightarrow \overline{\operatorname{Bun}}_{B} \times X$ defined in ([3], Section 3.1.3). For $\mu \in \Lambda_{G}^{\text {pos }} \cap \Lambda^{\sharp}$ we lift it to a map

$$
\tilde{i}_{\mu}: \overline{\operatorname{Bun}}_{\tilde{B}} \times X \hookrightarrow \overline{\operatorname{Bun}}_{\tilde{B}} \times X
$$

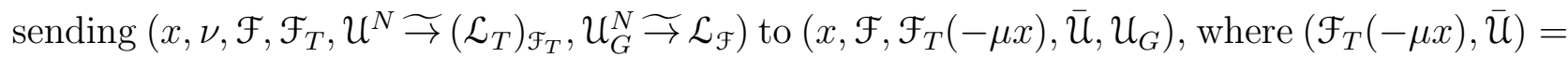
$m^{\mu}\left(\mathcal{F}_{T}, \mathcal{U}\right)$ is equipped with the induced inclusions

$$
\mathcal{L}_{\mathcal{F}_{T}(-\mu x)}^{\check{\lambda}} \hookrightarrow V_{\mathscr{F}}^{\check{\lambda}}
$$

Set for brevity ${ }_{\mu} \mathrm{IC}_{\zeta}=\tilde{i}_{\mu !}\left(\mathrm{IC}_{\zeta} \otimes \mathrm{IC}(X)\right)$.

Proposition 3.2. One has canonically

$$
\phi_{!} \mathrm{IC}(\bar{Z})_{\zeta} \widetilde{\rightrightarrows} \underset{\mu \in \Lambda_{G}^{\text {pos } \cap \Lambda^{\sharp}}}{\oplus} \mu \mathrm{IC}_{\zeta} \otimes V^{\nu}\left(\mu+w_{0}(\nu)\right)
$$

\subsubsection{Proof of Theorem 2.2.}

Lemma 3.2. 1) The maps $\tilde{\mathfrak{q}}\left({ }^{\prime} h_{G}\right)$ and $m^{w_{0}(\nu)}(\tilde{\mathfrak{q}} \times \mathrm{id}) \phi$ from $\bar{Z}$ to $\widetilde{\operatorname{Bun}}_{T}$ coincide.

2) For any $\mu \in \Lambda^{\sharp} \cap \Lambda_{G}^{\text {pos }}$ the diagram is canonically 2-commutative

$$
\begin{array}{ccc}
\overline{\operatorname{Bun}}_{\tilde{B}} \times X & \stackrel{\tilde{\mathfrak{q}} \times \mathrm{id}}{\rightarrow} & \widetilde{\operatorname{Bun}}_{T} \times X \\
\downarrow \tilde{i}_{\mu} & & \downarrow m^{\mu} \times \mathrm{id} \\
\overline{\operatorname{Bun}}_{\tilde{B}} \times X & \stackrel{\tilde{\mathfrak{q}} \times \mathrm{id}}{\rightarrow} & \widetilde{\operatorname{Bun}_{T}} \times X
\end{array}
$$

Let $K \in \mathrm{D}_{\zeta}\left(\widetilde{\operatorname{Bun}}_{T}\right)$. The complex $\left(\tilde{h}_{G}\right)^{*} \operatorname{Eis}(K) \otimes \mathrm{IC}^{\nu}$ over $\overline{\mathcal{H}}_{\tilde{G}}^{\nu}$ identifies with $\mathrm{IC}^{\nu} \otimes\left({ }^{\prime} \tilde{\mathfrak{p}}\right) !\left(\left({ }^{\prime} h_{G}\right)^{*} \tilde{\mathfrak{q}}^{*} K \otimes\left({ }^{\prime} h_{G}\right)^{*} \mathrm{IC}_{\zeta}\right)\left[-\operatorname{dim} \mathrm{Bun}_{T}\right] \stackrel{\rightarrow}{\rightarrow}$

$$
\left({ }^{\prime} \tilde{\mathfrak{p}}\right)_{!}\left(\left({ }^{\prime} h_{G}\right)^{*} \tilde{\mathfrak{q}}^{*} K \otimes \mathrm{IC}(\bar{Z})_{\zeta}\right)\left[\operatorname{dim} \operatorname{Bun}_{G}-\operatorname{dim} \operatorname{Bun}_{T}\right]
$$

So,

$$
\begin{aligned}
& \mathrm{H}_{G}^{\nu} \operatorname{Eis}(K) \widetilde{\rightrightarrows}(\tilde{\mathfrak{p}} \times \mathrm{id}) ! \phi_{!}\left(\left({ }^{\prime} h_{G}\right)^{*} \tilde{\mathfrak{q}}^{*} K \otimes \mathrm{IC}(\bar{Z})_{\zeta}\right)\left[-\operatorname{dim} \mathrm{Bun}_{T}\right] \widetilde{\rightrightarrows} \\
& (\tilde{\mathfrak{p}} \times \mathrm{id})_{!}\left((\tilde{\mathfrak{q}} \times \mathrm{id})^{*}\left(m^{w_{0}(\nu)}\right)^{*} K \otimes \phi_{!} \operatorname{IC}(\bar{Z})_{\zeta}\right)\left[-\operatorname{dim} \operatorname{Bun}_{T}\right]
\end{aligned}
$$


By Proposition 3.2, this identifies with the direct sum over $\mu \in \Lambda_{G}^{\text {pos }} \cap \Lambda^{\sharp}$ of

$$
\begin{aligned}
& (\tilde{\mathfrak{p}} \times \mathrm{id}) !\left((\tilde{\mathfrak{q}} \times \mathrm{id})^{*}\left(m^{w_{0}(\nu)}\right)^{*} K \otimes \tilde{i}_{\mu !}\left(\mathrm{IC}_{\zeta} \otimes \mathrm{IC}(X)\right)\right) \otimes V^{\nu}\left(\mu+w_{0}(\nu)\right)\left[-\operatorname{dim} \operatorname{Bun}_{T}\right] \widetilde{\rightrightarrows} \\
& (\tilde{\mathfrak{p}} \times \mathrm{id}) ! \tilde{i}_{\mu !}\left(\tilde{i}_{\mu}^{*}(\tilde{\mathfrak{q}} \times \mathrm{id})^{*}\left(m^{w_{0}(\nu)}\right)^{*} K \otimes\left(\mathrm{IC}_{\zeta} \otimes \mathrm{IC}(X)\right)\right) \otimes V^{\nu}\left(\mu+w_{0}(\nu)\right)\left[-\operatorname{dim} \operatorname{Bun}_{T}\right]
\end{aligned}
$$

By Lemma 3.2, we get

$$
\tilde{i}_{\mu}^{*}(\tilde{\mathfrak{q}} \times \mathrm{id})^{*}\left(m^{w_{0}(\nu)}\right)^{*} K \widetilde{\Im}(\tilde{\mathfrak{q}} \times \mathrm{id})^{*}\left(m^{\mu} \times \mathrm{id}\right)^{*}\left(m^{w_{0}(\nu)}\right)^{*} K \widetilde{\Im}(\tilde{\mathfrak{q}} \times \mathrm{id})^{*} \mathrm{H}_{T}^{\mu+w_{0}(\nu)}(K)[-1],
$$

because $m^{w_{0}(\nu)}\left(m^{\mu} \times \mathrm{id}\right)=m^{\mu+w_{0}(\nu)}$. So, (11) identifies with the sum over $\mu \in \Lambda_{G}^{\text {pos }} \cap \Lambda^{\sharp}$ of

$$
(\tilde{\mathfrak{p}} \times \mathrm{id})_{!}\left((\tilde{\mathfrak{q}} \times \mathrm{id})^{*} \mathrm{H}_{T}^{\mu+w_{0}(\nu)}(K) \otimes\left(\mathrm{IC}_{\zeta} \otimes \overline{\mathbb{Q}}_{\ell}\right)\right) \otimes V^{\nu}\left(\mu+w_{0}(\nu)\right)\left[-\operatorname{dim} \operatorname{Bun}_{T}\right] \widetilde{\rightarrow}
$$

$$
(\text { Eis } \otimes \mathrm{id}) \mathrm{H}_{T}^{\mu+w_{0}(\nu)}(K) \otimes V^{\nu}\left(\mu+w_{0}(\nu)\right)
$$

Indeed, by definition,

$$
(\text { Eis } \otimes \mathrm{id})(\mathcal{S})=(\tilde{\mathfrak{p}} \times \mathrm{id}) !\left((\tilde{\mathfrak{q}} \times \mathrm{id})^{*} \mathcal{S} \otimes\left(\mathrm{IC}_{\zeta} \otimes \overline{\mathbb{Q}}_{\ell}\right)\right)\left[-\operatorname{dim} \operatorname{Bun}_{T}\right]
$$

Theorem 2.2 is reduced to Proposition 3.2 .

3.3.3. Proof of Proposition [3.2. As in ([3], Section 3.3.1), we fix $x \in X$, let ${ }_{x} \bar{Z}$ be obtained from $\bar{Z}$ by the base change Spec $k \stackrel{x}{\rightarrow} X$. We make this base change in the basic diagram and get

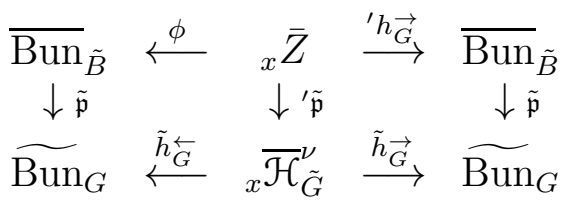

Let $\operatorname{IC}\left({ }_{x} \bar{Z}\right)_{\zeta}=\left.\operatorname{IC}(\bar{Z})_{\zeta}\right|_{x} \bar{Z}[-1]$. We will prove a version of Proposition 3.2 with $x$ fixed. The maps are denoted by the same letters as for $x$ varying.

For $\mu \in \Lambda_{G}^{\text {pos }}$ we have the stacks ${ }_{x, \geq \mu} \overline{\operatorname{Bun}}_{B},{ }_{x, \mu} \overline{\operatorname{Bun}}_{B}, x, \mu \operatorname{Bun}_{B}$ defined as in ([3], Section 3.3.2). We write

$$
{ }_{x, \geq \mu} \overline{\operatorname{Bun}}_{\tilde{B}}, \quad x, \mu \overline{\operatorname{Bun}}_{\tilde{B}}
$$

and so on for the stacks obtained by the base change $\overline{\operatorname{Bun}}_{\tilde{B}} \rightarrow \overline{\operatorname{Bun}}_{B}$ from the previous ones. Let $j_{\mu}:{ }_{x, \mu} \overline{\operatorname{Bun}}_{\tilde{B}} \hookrightarrow \overline{\operatorname{Bun}}_{\tilde{B}}$ be the natural embedding.

Proposition 3.3. The complex $j_{\mu}^{*} \phi_{!} \mathrm{IC}\left({ }_{x} \bar{Z}\right)_{\zeta}$ satisfies the following.

a) It lives in perverse degrees $\leq 0$.

b) The 0-th perverse cohomology sheaf of $j_{\mu}^{*} \phi_{!} \mathrm{IC}\left({ }_{x} \bar{Z}\right)_{\zeta}$ identifies with

$$
\left({ }_{\mu} \mathrm{IC}_{\zeta}\right) \otimes V^{\nu}\left(\mu+w_{0}(\nu)\right)
$$

For $\mu \in \Lambda_{G}^{\text {pos }}$ let $Z^{?, \mu}$ (resp., $Z^{\mu, ?}$ ) denote the preimage in ${ }_{x} \bar{Z}$ of ${ }_{x, \mu} \overline{\operatorname{Bun}}_{\tilde{B}}$ under ' $h_{G}$ (resp., $\phi)$. For $\mu, \mu^{\prime} \in \Lambda_{G}^{\text {pos }}$ let $Z^{\mu, \mu^{\prime}}=Z^{\mu, ?} \cap Z^{?, \mu^{\prime}}$. Recall that $Z^{\mu, \mu^{\prime}}$ is empty unless $\mu \geq \mu^{\prime}$.

If $\nu^{\prime} \in \Lambda^{+}$with $\nu^{\prime} \leq \nu$ then we write

$$
Z^{\mu, \mu^{\prime}, \nu^{\prime}}=Z^{\mu, \mu^{\prime}} \cap \cap^{\prime} \tilde{\mathfrak{p}}^{-1}\left({ }_{x} \mathcal{H}_{\tilde{G}}^{\nu^{\prime}}\right)
$$


Proposition 3.4. For $\mu, \mu^{\prime} \in \Lambda_{G}^{\text {pos }}$ and $\nu^{\prime} \in \Lambda^{+}$with $\nu^{\prime} \leq \nu$ let $K^{\mu, \mu^{\prime}, \nu^{\prime}} \in \mathrm{D}\left({ }_{x, \mu} \overline{\mathrm{Bun}}_{\tilde{B}}\right)$ be defined as

$$
\phi_{!}\left(\left.\mathrm{IC}\left({ }_{x} \bar{Z}\right)_{\zeta}\right|_{Z^{\mu, \mu^{\prime}, \nu^{\prime}}}\right)
$$

a) The complex $K^{\mu, \mu^{\prime}, \nu^{\prime}}$ is placed in perverse degrees $\leq 0$, the equality is strict unless $\mu^{\prime}=0$ and $\nu^{\prime}=\nu$.

b) The *-restriction of $K^{\mu, 0, \nu}$ to ${ }_{x, \mu} \overline{\operatorname{Bun}}_{\tilde{B}}-{ }_{x, \mu} \operatorname{Bun}_{\tilde{B}}$ is placed in perverse degrees $<0$.

c) The 0-th perverse cohomology sheaf of $K^{\mu, 0, \nu}$ over ${ }_{x, \mu} \operatorname{Bun}_{\tilde{B}}$ vanishes unless $\mu \in \Lambda^{\sharp} \cap \Lambda_{G}^{\text {pos }}$. In the latter case it identifies with

$$
\left.\left({ }_{\mu} \mathrm{IC}_{\zeta}\right)\right|_{x, \mu} \operatorname{Bun}_{\tilde{B}} \otimes V^{\nu}\left(\mu+w_{0}(\nu)\right)
$$

A version of ([3], Lemma 3.3.6) holds with obvious changes. For $\nu \in \Lambda^{+}$write $\overline{\mathrm{Gr}}_{\tilde{G}}^{\nu}=$ $\overline{\mathrm{Gr}}_{G}^{\nu} \times{ }_{\mathrm{Gr}_{G}} \widetilde{\mathrm{Gr}}_{G}$. For $\mu \in \Lambda$ the scheme $S_{G}^{\mu}$ is defined in ([3], Section 3.2.5). For $\mu \in \Lambda$ write

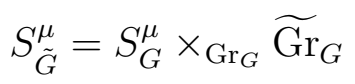

Lemma 3.3. (a) The $\operatorname{map}^{\prime} h_{G}: Z^{?, \mu^{\prime}} \rightarrow_{x, \mu^{\prime}} \overline{\operatorname{Bun}}_{\tilde{B}}$ is a locally trivial fibration with typical fibre $\overline{\mathrm{Gr}}_{\tilde{G}}^{-w_{0}(\nu)}$.

(b) The morphism ' $h_{G}: Z^{\mu, \mu^{\prime}, \nu^{\prime}} \rightarrow_{x, \mu^{\prime}} \overline{\operatorname{Bun}}_{\tilde{B}}$ identifies using the notations of (a) with a subfibration with typical fibre

$$
\operatorname{Gr}_{\tilde{G}}^{-w_{0}\left(\nu^{\prime}\right)} \cap S_{\tilde{G}}^{-w_{0}(\nu)-\mu+\mu^{\prime}}
$$

(c) The map $\phi: Z^{\mu, \mu^{\prime}, \nu^{\prime}} \rightarrow{ }_{x, \mu} \overline{\operatorname{Bun}}_{\tilde{B}}$ is a locally trivial fibration with fibre

$$
\operatorname{Gr}_{\tilde{G}}^{\nu^{\prime}} \cap S_{\tilde{G}}^{\mu-\mu^{\prime}+w_{0}(\nu)}
$$

Proof of Proposition 3.4 The complex $\left.\operatorname{IC}\left({ }_{x} \bar{Z}\right)_{\zeta}\right|_{Z^{\text {? }}, \mu^{\prime}}$ is a twisted external product

$$
\left.\mathcal{A}_{\varepsilon}^{-w_{0}(\nu)} \tilde{\otimes} \mathrm{IC}_{\zeta}\right|_{x, \mu^{\prime}} \overline{\operatorname{Bun}}_{\tilde{B}}
$$

with the notations of Lemma 3.3(a). So, $\left.\operatorname{IC}\left({ }_{x} \bar{Z}\right)_{\zeta}\right|_{Z^{\mu, \mu^{\prime}, \nu^{\prime}}}$ is a twisted external product

$$
\left.\left.\mathcal{A}_{\mathcal{E}}^{-w_{0}(\nu)}\right|_{\operatorname{Gr}_{\tilde{G}}^{-w_{0}\left(\nu^{\prime}\right)} \cap S_{\tilde{G}}^{-w_{0}(\nu)-\mu+\mu^{\prime}}} \tilde{\otimes} \mathrm{IC}_{\zeta}\right|_{x, \mu^{\prime}} \overline{\operatorname{Bun}}_{\tilde{B}}
$$

The complex $\left.\mathrm{IC}_{\zeta}\right|_{x, \mu^{\prime}} \overline{\operatorname{Bun}}_{\tilde{B}}$ is placed in perverse degrees $\leq 0$, and the inequality is strict unless $\mu^{\prime}=0$. Further,

$$
\left.\mathcal{A}_{\varepsilon}^{-w_{0}(\nu)}\right|_{\operatorname{Gr}_{\tilde{G}}^{-w_{0}\left(\nu^{\prime}\right)}}
$$

is a constant complex placed in perverse degrees $<0$ unless $\nu^{\prime}=\nu$. Now exactly as in ([3], Section 3.3.7) one proves a). This only uses the following. For a morphism $f: Y_{1} \rightarrow Y_{2}$ such that the maximal dimension of the fibres of $f$ is $\leq d$ and a perverse sheaf $F$ on $Y_{1}$ the complex $f_{!} F$ is placed in perverse degrees $\leq d$. The point b) is proved similarly (as in [3], Section 3.3.7).

c) Let ${ }^{0} Z^{\mu, 0, \nu}$ be the preimage of $\operatorname{Bun}_{\tilde{B}} \subset x, 0 \overline{\operatorname{Bun}}_{\tilde{B}}$ under ${ }^{\prime} h_{G}$. One has

$$
\left.K^{\mu, 0, \nu}\right|_{x, \mu} \operatorname{Bun}_{\tilde{B}}=\phi_{!}\left(\left.\mathrm{IC}\left({ }_{x} \bar{Z}\right)_{\zeta}\right|^{0} Z^{\mu, 0, \nu}\right)
$$


A point of ${ }_{x, \mu} \operatorname{Bun}_{\tilde{B}}$ is given by $\left(\mathcal{F}_{T}, \mathcal{F}, \mathcal{U}^{N} \widetilde{\Im}\left(\mathcal{L}_{T}\right)_{\mathcal{F}_{T}}, \mathcal{U}_{G}^{N} \widetilde{\Im} \mathcal{L}_{\mathcal{F}}\right)$. Let

$$
(a, b) \in \mu_{N}(k) \times \mu_{N}(k) \subset \operatorname{Aut}\left(\mathcal{U}_{G}\right) \times \operatorname{Aut}(\mathcal{U})
$$

acting trivially on $\mathcal{F}_{T}, \mathcal{F}$ then $(a, b)$ acts on (13) as $\zeta\left(\frac{a}{b}\right)$. One shows as in ([17], Lemma 11) that any bounded complex in $\mathrm{D}\left({ }_{x, \mu} \operatorname{Bun}_{\tilde{B}}\right)$, on which $(a, b)$ acts as $\zeta\left(\frac{a}{b}\right)$, vanishes unless $\mu \in \Lambda^{\sharp}$. Assume $\mu \in \Lambda^{\sharp}$.

The complex $\operatorname{IC}\left({ }_{x} \bar{Z}\right)_{\zeta} \mid{ }_{0} Z^{\mu, 0, \nu}$ is the twisted external product with the notations of Lemma $\underline{3.3}$ (a)

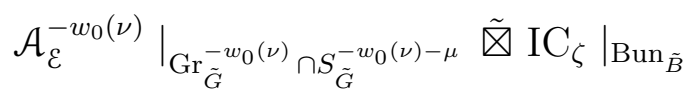

and $\left.\mathcal{A}_{\mathcal{E}}^{-w_{0}(\nu)}\right|_{\mathrm{Gr}_{\tilde{G}}^{-w_{0}(\nu)}} \widetilde{\rightrightarrows} E_{\mathcal{E}}^{-w_{0}(\nu)}[\langle\nu, 2 \check{\rho}\rangle]$.

Now in the notation of Lemma 3.3)(c), the map $\phi:{ }^{0} Z^{\mu, 0, \nu} \rightarrow{ }_{x, \mu} \operatorname{Bun}_{\tilde{B}}$ and (14) becomes a locally trivial fibration

$$
\left(\operatorname{Gr}_{\tilde{G}}^{\nu} \cap S_{\tilde{G}}^{\mu+w_{0}(\nu)}\right) \tilde{\times}_{x, \mu} \operatorname{Bun}_{\tilde{B}} \rightarrow{ }_{x, \mu} \operatorname{Bun}_{\tilde{B}}
$$

and the complex $\left(E_{\varepsilon}^{\nu}\right)^{*} \tilde{\otimes}\left(\left.{ }_{\mu} \mathrm{IC}_{\zeta}\right|_{x, \mu} \operatorname{Bun}_{\tilde{B}}\right)[2\langle\mu, \check{\rho}\rangle]$ on the source.

Applying now Proposition 3.1 (with the character $\zeta$ replaced by $\zeta^{-1}$ ), we see that the 0 -th perverse cohomology sheaf of $\left.K^{\mu, 0, \nu}\right|_{x, \mu} \operatorname{Bun}_{\tilde{B}}$ identifies with $\left.\left({ }_{\mu} \mathrm{IC}_{\zeta}\right)\right|_{x, \mu} \operatorname{Bun}_{\tilde{B}} \otimes V^{\nu}\left(\mu+w_{0}(\nu)\right)$.

So, Proposition 3.3 is also proved. This concludes the proof of Proposition 3.2 (and hence of Theorem 2.2).

3.4. Proof of Theorem [2.1. An analog of ([3], Theorem 5.1.5) holds in our situation.

Proposition 3.5. The perverse sheaf $\mathrm{IC}_{\zeta}$ is $U L A$ with respect to $\tilde{\mathfrak{q}}: \overline{\operatorname{Bun}}_{\tilde{B}} \rightarrow \widetilde{\operatorname{Bun}}_{T}$.

Proof Pick $\lambda_{1}, \ldots, \lambda_{r} \in \Lambda^{\sharp,+}$ such that they form a base in $\Lambda^{\sharp} \otimes_{\mathbb{Z}} \mathbb{Q}$. Let $m \geq 2 g-1$, let $\triangle \hookrightarrow X^{m r}$ denote the divisor of diagonals.

Let $\mathcal{H}_{\tilde{G}}^{?}$ be the stack classifying

$$
\left\{x_{1,1}, \ldots, x_{1, m}, x_{2,1}, \ldots, x_{r, 1}, \ldots, x_{r, m}\right\} \in X^{m r}-\Delta,\left(\mathcal{F}, \mathcal{U}_{G}\right),\left(\mathcal{F}^{\prime}, \mathcal{U}_{G}^{\prime}\right) \in \widetilde{\operatorname{Bun}}_{G}
$$

an isomorphism $\beta:\left.\mathcal{F} \widetilde{\rightarrow} \mathcal{F}^{\prime}\right|_{X-\left\{x_{1,1}, \ldots, x_{r, m}\right\}}$ such that for all $i, j, \mathcal{F}$ is in the position $\lambda_{i}$ with respect to $\mathcal{F}^{\prime}$ at $x_{i, j}$.

Let $h_{G}^{\overleftarrow{G}}, h_{G}: \mathcal{H}_{\tilde{G}}^{?} \rightarrow{\widetilde{\operatorname{Bun}_{G}}}$ denote the projections sending the above point to $\left(\mathcal{F}, \mathcal{U}_{G}\right)$ and $\left(\mathcal{F}^{\prime}, \mathcal{U}_{G}^{\prime}\right)$ respectively. Let $\pi: \mathcal{H}_{\tilde{G}}^{?} \rightarrow X^{m r}-\triangle$ be the projection.

Let $\bar{Z}=\mathcal{H}_{\tilde{G}}^{?} \times{\widetilde{\operatorname{Bun}_{G}}}_{\operatorname{Bun}_{\tilde{B}}}$, where we used $h_{G}$ to define the fibred product. Let $\phi: \bar{Z} \rightarrow$ $\overline{\operatorname{Bun}}_{\tilde{B}} \times\left(X^{m r}-\triangle\right)$ be the map defined as in Lemma 3.1, we get a commutative diagram

$$
\begin{array}{ccccc}
\overline{\operatorname{Bun}}_{\tilde{B}} \times\left(X^{m r}-\triangle\right) & \stackrel{\phi}{\longleftarrow} & \bar{Z} & \stackrel{\prime h \vec{G}_{\longrightarrow}}{\longrightarrow} & \overline{\operatorname{Bun}}_{\tilde{B}} \\
\downarrow \tilde{\mathfrak{p}} \times \mathrm{id} & & \downarrow & & \downarrow \tilde{\mathfrak{p}} \\
\widetilde{\operatorname{Bun}}_{G} \times\left(X^{m r}-\triangle\right) & \stackrel{h_{G}^{\leftarrow} \times \pi}{\longleftarrow} & \mathcal{H}_{\tilde{G}}^{?} & \stackrel{h_{G}}{\longrightarrow} & \widetilde{\operatorname{Bun}}_{G}
\end{array}
$$


Let $A J: X^{m r}-\triangle \rightarrow \operatorname{Bun}_{T^{\sharp}}$ be the map sending $\left(x_{i, j}\right)$ to

$$
\mathcal{F}_{T^{\sharp}}^{0}\left(\sum_{i, j} \lambda_{i} x_{i, j}\right),
$$

it is smooth. The composition $\bar{Z}^{\prime} \stackrel{h \rightarrow}{\rightarrow} \overline{\operatorname{Bun}}_{\tilde{B}} \stackrel{\tilde{\mathfrak{q}}}{\rightarrow} \widetilde{\operatorname{Bun}}_{T}$ equals the composition

$$
\bar{Z} \stackrel{\phi}{\rightarrow} \overline{\operatorname{Bun}}_{\tilde{B}} \times\left(X^{m r}-\triangle\right) \stackrel{\text { id } \times A J}{\longrightarrow} \overline{\operatorname{Bun}}_{\tilde{B}} \times \operatorname{Bun}_{T^{\sharp}} \stackrel{m_{\tilde{\mathfrak{q}}}}{\longrightarrow} \widetilde{\operatorname{Bun}}_{T}
$$

Here $m_{\tilde{\mathfrak{q}}}$ denotes the composition $\overline{\operatorname{Bun}}_{\tilde{B}} \times \operatorname{Bun}_{T^{\sharp}} \stackrel{\tilde{\mathfrak{q}} \times \mathrm{id}}{\rightarrow}{\widetilde{\operatorname{Bun}_{T}}} \times \operatorname{Bun}_{T^{\sharp}} \stackrel{a}{\rightarrow} \widetilde{\operatorname{Bun}}_{T}$, where $a$ is the action map defined in ([17], Section 5.2.3).

Define $Z \subset \bar{Z}$ as the open substack classifying $\left(x_{i, j}, \mathcal{F}, \mathcal{U}_{G}, \mathcal{F}^{\prime}, \mathcal{U}_{G}^{\prime}, \mathcal{F}_{T}^{\prime}, \mathcal{U}^{\prime}, \nu^{\prime}\right) \in \bar{Z}$ such that for all $\check{\lambda} \in \check{\Lambda}^{+}$the following holds:

a) The map $\nu^{\prime \check{\lambda}}: \mathcal{L}_{\mathcal{F}_{T}^{\prime}}^{\check{\lambda}} \hookrightarrow \mathcal{V}_{\mathcal{F}_{G}^{\prime}}^{\check{\lambda}}$ has no zero at $x_{i, j}$.

b) The map $\nu^{\check{\lambda}}: \mathcal{L}_{\mathcal{F}_{T}^{\prime}}^{\check{L}}\left(-\sum_{i, j}\left\langle\lambda_{i}, \check{\lambda}\right\rangle x_{i, j}\right) \hookrightarrow \mathcal{V}_{\mathcal{F}_{G}}^{\check{\lambda}}$ has no zero at each $x_{i, j}$.

As in ([3], Theorem 5.1.5), the map ${ }^{\prime} h{ }_{G}: Z \rightarrow \overline{\operatorname{Bun}}_{\tilde{B}}$ is smooth and surjective, $\phi: Z \rightarrow$ $\overline{\operatorname{Bun}}_{\tilde{B}} \times\left(X^{m r}-\triangle\right)$ is smooth. We get a diagram

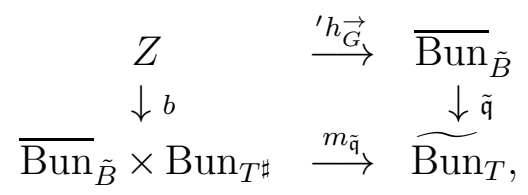

where $b=(\mathrm{id} \times A J) \circ \phi$, so $b$ is smooth. It is easy to see that there is a rank one local system $\mathcal{E}$ on $Z$ with $\mathcal{E}^{N} \widetilde{\rightarrow} \overline{\mathbb{Q}}_{\ell}$ and an isomorphism

$$
\left({ }^{\prime} h_{G}\right)^{*} \mathrm{IC}_{\zeta} \widetilde{\rightarrow} \mathcal{E} \otimes b^{*}\left(\mathrm{IC}_{\zeta} \otimes \mathrm{IC}\left(\mathrm{Bun}_{T^{\sharp}}\right)\right)
$$

So, it suffices to show that $\mathrm{IC}_{\zeta} \otimes \mathrm{IC}\left(\mathrm{Bun}_{T^{\sharp}}\right)$ is ULA with respect to $m_{\tilde{\mathfrak{q}}}$. Note that $m_{\tilde{\mathfrak{q}}}$ is the composition

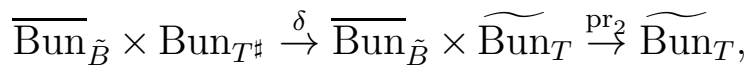

where $\delta$ composed with the projection to $\overline{\operatorname{Bun}}_{\tilde{B}}$ (resp., to $\widetilde{\operatorname{Bun}}_{T}$ ) is $\operatorname{pr}_{1}$ (resp., $m_{\tilde{q}}$ ). Since $\widetilde{\operatorname{Bun}}_{T}$ is smooth $\mathrm{IC}_{\zeta} \otimes \mathrm{IC}\left(\widetilde{\operatorname{Bun}}_{T}\right)$ is ULA over $\widetilde{\operatorname{Bun}}_{T}$. Since $\delta$ is smooth, our claim follows from ([3], 5.1.2 (2)). See Remark 3.1] below.

Remark 3.1. Let $f: Y \rightarrow Z$ be a morphism of schemes with $Z$ smooth. Let $H$ be a smooth group scheme acting on $Z$, assume the stabilizor in $H$ of any point of $Z$ is smooth. Assume that for any $k$-point $z \in Z$ the map $H \rightarrow Z, h \mapsto h z$ is smooth. Let $K \in \mathrm{D}(Y)$. Let $m_{f}$ denote the composition $H \times Y \stackrel{\text { id } \times f}{\rightarrow} H \times Z \stackrel{a}{\rightarrow} Z$, where $a$ is the action map. Then $\overline{\mathbb{Q}}_{\ell} \otimes K$ is ULA with respect to $m_{f}$. Indeed, $m_{f}$ is written as the composition $H \times Y \stackrel{\delta}{\rightarrow} Z \times Y \stackrel{\text { pr }_{1}}{\rightarrow} Z$, where $\delta$ composed with the projection to $Y$ is $\operatorname{pr}_{2}$. The map $\delta$ is smooth, because it is obtained by base change from the map $H \times Z \rightarrow Z \times Z,(h, z) \mapsto(h z, z)$.

Theorem 2.1 follows from Proposition 3.5 by applying ([3], Section 5.1.2). 
3.5. Some gradings. The center $Z(G)$ acts on $\operatorname{Bun}_{G}$ by 2-automorphisms, namely $z \in$ $Z(G)$ yields an automorphism $\mathcal{F} \rightarrow \mathcal{F}, f \mapsto f z$ of $\mathcal{F} \in \operatorname{Bun}_{G}$. This automorphism acts trivially on $\mathfrak{g}_{\mathcal{F}}$. We let $Z(G)$ act on $\widetilde{\operatorname{Bun}}_{G}$ by 2 -automorphisms so that for $(\mathcal{F}, \mathcal{U}) \in \widetilde{\operatorname{Bun}}_{G}$, $z \in Z(G)$ acts naturally on $\mathcal{F}$ and trivially on $\mathcal{U}$. For a character $\chi: Z(G) \rightarrow \overline{\mathbb{Q}}_{\ell}^{*}$ we get the full triangulated subcategory $\mathrm{D}_{\zeta, \chi}\left(\widetilde{\operatorname{Bun}}_{G}\right) \subset \mathrm{D}_{\zeta}\left(\widetilde{\operatorname{Bun}}_{G}\right)$ of objects on which $Z(G)$ acts by $\chi$.

Write $C^{*}\left(\check{G}_{n}\right)$ for the cocenter of $\check{G}_{n}$, the quotient of $\Lambda^{\sharp}$ by the roots lattice of $\check{G}_{n}$. One has canonically $\operatorname{Hom}\left(Z\left(\check{G}_{n}\right), \overline{\mathbb{Q}}_{\ell}^{*}\right) \widetilde{\rightarrow} C^{*}\left(\check{G}_{n}\right)$. If $\mu \in \Lambda^{\sharp,+}$ then $Z\left(\check{G}_{n}\right)$ acts on $V^{\mu}$ by the character, which is the image of $\mu$ in $C^{*}\left(\check{G}_{n}\right)$.

There is a natural map $\xi: C^{*}\left(\check{G}_{n}\right) \rightarrow \operatorname{Hom}\left(Z(G), \mu_{n}(k)\right)$ sending $\nu \in \Lambda^{\sharp}$ to the character $\frac{\iota(\nu)}{n}$. The latter sends $z \in Z(G)$ to $\frac{\iota(\nu)}{n}(z)$. If $\alpha$ is a simple coroot of $G$ then let $\delta$ denote the denominator of $\frac{\iota(\alpha, \alpha)}{2 n}$. Recall that $\delta \alpha$ is the corresponding simple root of $\check{G}_{n}$ ([9], Theorem 2.9). Since $\frac{\iota(\delta \alpha)}{n}$ lies in the roots lattice of $G, \frac{\iota(\delta \alpha)}{n}(z)=1$ for $z \in Z(G)$ by Remark 3.2 below. Thus, $\xi$ is correctly defined. Write $C^{*}(G)_{n}$ for the $n$-torsion subgroup of $C^{*}(G)$.

Lemma 3.4. The map $C^{*}\left(\check{G}_{n}\right) \rightarrow C^{*}(G)$ sending $\nu \in \Lambda^{\sharp}$ to $\frac{\iota(\nu)}{n}$ is injective and takes values in $C^{*}(G)_{n}$. The above map $C^{*}\left(\check{G}_{n}\right) \rightarrow C^{*}(G)_{n}$ is not always surjective 1

Proof Let us check this case by case for all simple simply-connected groups.

1) If $G$ is simply-laced then for each simple coroot $\alpha$ of $G$ the corresponding root of $\breve{G}_{n}$ is $n \alpha$. Besides, $\iota(\alpha)=\check{\alpha}$ for each simple coroot $\alpha$ of $G$. So, if $\lambda \in \Lambda^{\sharp}$ and $\iota(\lambda)$ lies in $n \check{Q}$, where $\check{Q}$ is the roots lattice of $G$ then $\lambda \in n \Lambda$. Our claim follows in this case. For $G$ simply-laced the map $C^{*}\left(\check{G}_{n}\right) \rightarrow C^{*}(G)$ identifies $C^{*}\left(\check{G}_{n}\right)$ with the $n$-torsion subgroup in $C^{*}(G)$.

2) If $G=\mathbb{S p}_{2 m}$ then the nontrivial case is $n$ even. In this case let $\alpha_{i}$ be the standard simple coroots, so $\iota\left(\alpha_{i}\right)=2 \check{\alpha}_{i}$ for $i<m$ and $\iota\left(\alpha_{m}\right)=\check{\alpha}_{m}$. In this case $\Lambda^{\sharp}=\frac{n}{2} \Lambda$, the simple roots of $\check{G}_{n}$ are $\left\{\frac{n}{2} \alpha_{i}, i<m\right.$ and $\left.n \alpha_{m}\right\}$. So, $\frac{n}{2} \alpha_{m} \in C^{*}\left(\check{G}_{n}\right) \widetilde{\rightarrow} / 2 \mathbb{Z}$ is a generator. Since $\frac{\check{\alpha}_{n}}{2}$ is not in the roots lattice of $G$, our claim follows, the map under consideration is actually an isomorphism.

3) If $G=\operatorname{Spin}_{2 m+1}$ with $m \geq 2$ then the only nontrivial case is $n$ even and $n m / 2$ even. In this case $C^{*}\left(\check{G}_{n}\right) \stackrel{\rightarrow}{\rightarrow} / 2 \mathbb{Z}$. Let $\alpha_{i}$ denote the standard simple coroots. Then $\iota\left(\alpha_{i}\right)=\check{\alpha}_{i}$ for $i<m$, and $\iota\left(\alpha_{m}\right)=2 \check{\alpha}_{m}$. The simple roots of $\check{G}_{n}$ are $n \alpha_{i}, i<m$ and $\frac{n}{2} \alpha_{m}$. The roots lattice of $\check{G}_{n}$ is $n \Lambda$, where $\Lambda=\left\{\left(a_{1}, \ldots, a_{m}\right) \mid \sum a_{i}=0 \bmod 2\right\}$. So, $\left(\frac{n}{2}, \ldots, \frac{n}{2}\right) \in \Lambda^{\sharp}$ generates $\Lambda^{\sharp} / n \Lambda$. The roots lattice of $G$ is $\mathbb{Z}^{n}$, and $\frac{\iota(\nu)}{n}$ sends the above generator to $\left(\frac{1}{2}, \ldots, \frac{1}{2}\right)$, which is not in $\mathbb{Z}^{n}$. The map under consideration is an isomorphism in this case.

However, if $n$ is even and $n m / 2$ is odd then the map under consideration $C^{*}\left(\check{G}_{n}\right) \rightarrow C^{*}(G)$ is not surjective!

4) For $G_{2}$ and $F_{4}$ the claim is trivial, as the center is trivial.

\footnotetext{
${ }^{1}$ The only case when it is not surjective is indicated in part 3) of the proof.
} 
Proposition 3.6. Let $\nu \in \Lambda^{\sharp,+}$ and $K \in \mathrm{D}_{\zeta}\left(\widetilde{\operatorname{Bun}_{G}}\right)$. Assume that $Z(G)$ acts on $K$ (by functoriality from the above 2-action on $\widetilde{\mathrm{Bun}_{G}}$ ) by a character $\chi: Z(G) \rightarrow \overline{\mathbb{Q}}_{\ell}^{*}$. Then $z \in Z(G)$ acts on $\mathrm{H}_{G}^{\nu}(K)$ as $\chi(z) \zeta\left(\frac{i(\nu)}{n}(z)\right)$.

Proof Recall that a point of $\overline{\mathcal{H}}_{\tilde{G}}^{\nu}$ is given by $\left(\mathcal{F}, \mathcal{F}^{\prime}, \beta:\left.\mathcal{F} \rightarrow \mathcal{F}^{\prime}\right|_{X-x}\right) \in \overline{\mathcal{H}}_{G}^{\nu}$ and $\mathcal{U}, \mathcal{U}^{\prime}$. Let $Z(G)$ act on $\overline{\mathcal{H}}_{\tilde{G}}^{\nu}$ by 2 -automorphisms so that it acts naturally on $\mathcal{F}, \mathcal{F}^{\prime}$ and trivially on $\mathcal{U}, \mathcal{U}^{\prime}$. Let us show that $z \in Z(G)$ acts on $\operatorname{IC}^{\nu}$ as $\zeta\left(\frac{i(\nu)}{n}(z)\right)$.

Consider the open substack $\mathcal{H}_{\tilde{G}}^{\nu} \subset \overline{\mathcal{H}}_{\tilde{G}}^{\nu}$. There is a line bundle, say $\mathcal{B}$, on $\mathcal{H}_{G}^{\nu}$ such that $\mathcal{B}^{N}$ is canonically the line bundle with fibre $\mathcal{L}_{\mathcal{F}} \otimes \mathcal{L}_{\mathcal{F}^{\prime}}^{-1}$ at $\left(\mathcal{F}, \mathcal{F}^{\prime}, \beta\right) \in \mathcal{H}_{G}^{\nu}$. The line bundle is uniquely defined, as the Picard group is torsion free. For $z \in Z(G)$ consider the 2-automorphism of $\mathcal{H}_{G}^{\nu}$ acting as $z$ on $\mathcal{F}, \mathcal{F}^{\prime}$. Then $z$ acts on $\mathcal{B}$ as $\frac{i(\nu)}{n}\left(z^{-1}\right)$. Actually, $\frac{i(\nu)}{n}\left(z^{-1}\right) \in \mu_{n}(k)$, because of Remark 3.2 below. We have an isomorphism

$$
\eta: B\left(\mu_{N}\right) \times\left(\mathcal{H}_{G}^{\nu} \times \mathrm{Bun}_{G} \widetilde{\operatorname{Bun}_{G}}\right) \widetilde{\rightarrow} \mathcal{H}_{\tilde{G}}^{\nu}
$$

where we used $h_{G}$ to define the fibred product in parentheses. It sends a collection $(\beta$ :

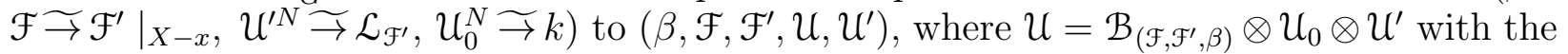
induced isomorphism $\mathcal{U}^{N} \underset{\rightarrow}{\rightarrow} \mathcal{L}_{\mathcal{F}}$. The perverse sheaf $\mathrm{IC}^{\nu}$ is the intermediate extension of

$$
\eta_{*}\left(\mathcal{L}_{\zeta} \otimes \mathrm{IC}\left(\mathcal{H}_{G}^{\nu} \times_{\mathrm{Bun}_{G}} \widetilde{\mathrm{Bun}_{G}}\right)\right)
$$

So, our 2-automorphism $z \in Z(G)$ of $\overline{\mathcal{H}}_{\tilde{G}}^{\nu}$ acts on $\mathrm{IC}^{\nu}$ as $\zeta\left(\frac{i(\nu)}{n}(z)\right)$. The group $Z(G)$ acts by the above 2-automorphisms on the diagram

$$
\widetilde{\operatorname{Bun}}_{G} \times X \stackrel{\tilde{h}_{\overleftarrow{G}}^{\leftarrow} \times \pi}{\overline{\mathcal{H}}_{\tilde{G}}^{\nu}} \tilde{h}_{\vec{G}}^{\vec{G}} \widetilde{\operatorname{Bun}_{G}},
$$

our assertion follows.

Remark 3.2. i) For any $\nu \in \Lambda$ the element $\iota(\nu)$ lies in the root lattice of $G$. So, if $z \in Z(G)$ then $\iota(\nu)(z)=1$.

ii) Recall that $G$ is simple, simply-connected. Write $Z(G)_{n}$ for the $n$-torsion subgroup of $Z(G)$. If $G$ is simply-laced then $\mathscr{G}_{n}$ is isomorphic to the Langlands dual to $G / Z(G)_{n}$. This was also observed by Savin in [24].

3.5.1. The group $T$ acts naturally on $\operatorname{Bun}_{T}$ by 2-automorphisms, under this action $t \in T$ acts on $\left.\mathcal{L}_{T}\right|_{\operatorname{Bun}_{T}^{\mu}}$ by the character $-\kappa(\mu)(t)$. In partucular, $Z(G) \subset T$ acts on $\operatorname{Bun}_{T}$ by 2-automorphisms, and acts trivially on $\mathcal{L}_{T}$. We let $Z(G)$ act on $\widetilde{\operatorname{Bun}}_{T}$ by 2-automorphisms, so that $z \in Z$ acts on $\left(\mathcal{F}_{T}, \mathcal{U}\right)$ as $z$ on $\mathcal{F}_{T}$ and trivially on $\mathcal{U}$. We also let $Z(G)$ act on $\overline{\operatorname{Bun}}_{\tilde{B}}$ by 2-automorphisms so that $z \in Z$ acts on $\left(\mathcal{F}_{T}, \mathcal{F}, \mathcal{U}^{N} \widetilde{\rightrightarrows}\left(\mathcal{L}_{T}\right)_{\mathcal{F}_{T}}, \mathcal{U}_{G}^{N} \widetilde{\rightarrow} \mathcal{L}_{\mathcal{F}}\right)$ as $z$ on $\mathcal{F}_{T}, \mathcal{F}_{G}$ and trivially on $\mathcal{U}, \mathcal{U}_{G}$. The diagram (3) is equivariant with respect to this 2 -action. The group $Z(G)$ acts trivially on $\mathrm{IC}_{\zeta}$. So, given $\mathcal{S} \in \mathrm{D}_{\zeta}\left(\widetilde{\operatorname{Bun}}_{T}\right)$, if $Z(G)$ acts on $\mathcal{S}$ by a character $\chi: Z(G) \rightarrow \overline{\mathbb{Q}}_{\ell}^{*}$ then $Z(G)$ acts on $\operatorname{Eis}(\mathcal{S})$ also by $\chi$.

Lemma 3.5. Let $\nu \in \Lambda^{\sharp}, K \in \mathrm{D}_{\zeta}\left(\widetilde{\operatorname{Bun}_{T}}\right)$. Then $z \in Z(G)$ acts on $K$ as $\zeta\left(\frac{\iota(\nu)}{n}\left(z^{-1}\right)\right)$. 
Proof

Step 1. The group $T$ acts trivially on $\left.\mathcal{L}_{T}\right|_{\operatorname{Bun}_{T}^{0}}$. So, $T$ acts by 2 -automorphisms of $\widetilde{\operatorname{Bun}}_{T}^{0}$, namely, $t \in T$ acts as $t$ on $\mathcal{F}$ and trivially on $\mathcal{U}$. For any $K \in \mathrm{D}_{\zeta}\left(\widetilde{\operatorname{Bun}}_{T}^{0}\right), T$ acts trivially on $K$, so $Z(G)$ also acts trivially on $K$.

Step 2. Pick $x \in X$, let ${ }_{x} m^{\nu}: \widetilde{\operatorname{Bun}}_{T} \rightarrow \widetilde{\operatorname{Bun}}_{T}$ be the restriction of $m^{\nu}$ to $x$. Then ${ }_{x} m^{\nu}$ is an isomoprhism. From (8) we see if $z \in Z(G)$ acts on some $\mathcal{S} \in \mathrm{D}_{\zeta}\left(\widetilde{\operatorname{Bun}_{T}}\right)$ as $\chi(z)$ then $z$ acts on $\left({ }_{x} m^{\nu}\right) * \mathcal{S}$ as $\chi(z) \zeta\left(\frac{\iota(\nu)}{n}(z)\right)$.

3.6. Towards the functional equation. The $W$-action on $\Lambda$ perserves $\Lambda^{\sharp}$, so $W$ acts on the left on $T^{\sharp}$ naturally. For $w \in W$ we denote also by $w: \operatorname{Bun}_{T^{\sharp}} \rightarrow \operatorname{Bun}_{T^{\sharp}}, \mathcal{F} \mapsto{ }^{w} \mathcal{F}$ the extension of scalars map with respect to the left action map $w: T^{\sharp} \rightarrow T^{\sharp}$. We also let $W$ act on the left on $\check{\Lambda}$, so $w \check{\lambda}=\check{\lambda} \circ w^{-1} \in \check{\Lambda}$. For $\check{\lambda} \in \check{\Lambda}, \mathcal{F} \in \operatorname{Bun}_{T}$ we get $\mathcal{L}_{(w \mathcal{A})}^{\check{I}} \widetilde{\mathcal{L}_{\mathcal{F}} w^{-1} \check{\lambda}}$ canonically. For $w_{i} \in W, \mathcal{F} \in \operatorname{Bun}_{T^{\sharp}}$ one has ${ }^{w_{2}}\left({ }^{w_{1}} \mathcal{F}\right) \widetilde{\rightrightarrows}{ }^{w_{2} w_{1}} \mathcal{F}$ naturally.

We let also $W$ act on the left on $\check{T}^{\sharp}$. For a $\check{T}^{\sharp}$-local system $E$ on $X$ we denote by ${ }^{w} E$ the extension of scalars of $E$ with respect to $w: \check{T}^{\sharp} \rightarrow \check{T}^{\sharp}$. So, for $\mu \in \Lambda^{\sharp}$ we get $\left({ }^{w} E\right)^{\mu} \widetilde{\rightarrow}(E)^{w^{-1} \mu}$ as local systems on $X$. For $w \in W$ and the map $w^{-1}: \operatorname{Bun}_{T^{\sharp}} \rightarrow \operatorname{Bun}_{T^{\sharp}}$ we then get $\left(w^{-1}\right)^{*} A E \widetilde{\rightarrow} A\left({ }^{w} E\right)$ canonically.

Let $W$ act naturally on the left on $T$. For $w \in W$ write ${ }^{w} \mathcal{F}$ for the extension of scalars of $\mathcal{F} \in \operatorname{Bun}_{T}$ under $w: T \rightarrow T$. Write $w: \operatorname{Bun}_{T} \rightarrow \operatorname{Bun}_{T}$ for the $\operatorname{map}^{\mathcal{F}} \mapsto{ }^{w} \mathcal{F}$, this is a left action of $W$ on $\operatorname{Bun}_{T}$. The line bundle $\mathcal{L}_{T}$ is naturally $W$-equivariant. Write also $w: \widetilde{\operatorname{Bun}}_{T} \rightarrow \widetilde{\operatorname{Bun}}_{T}$ for the map $\left(\mathcal{F}, \mathcal{U}, \mathcal{U}^{N} \widetilde{\Im}\left(\mathcal{L}_{T}\right)_{\mathcal{F}}\right) \mapsto\left({ }^{w} \mathcal{F}, \mathcal{U}\right)$ with the induced isomorphism $\mathcal{U}^{N} \widetilde{\Im}\left(\mathcal{L}_{T}\right)_{w \mathcal{F}}$. This defines a left action of $W$ on $\widetilde{\operatorname{Bun}}_{T}$.

For a $\check{T}^{\sharp}$-local system $E$ on $X$ let $\mathcal{K}_{E} \in \mathrm{D}_{\zeta}\left(\widetilde{\operatorname{Bun}}_{T}\right)$ be the $E$-Hecke eigensheaf constructed in ([17], Proposition 2.2), this is a local system over the components $\widetilde{\operatorname{Bun}}_{T}^{\mu}, \mu \in \Lambda^{\sharp}$.

Proposition 3.7. For $w \in W$ there is an isomorphism $\left(w^{-1}\right)^{*} \mathcal{K}_{E} \Im \mathcal{K}_{\left(w_{E)}\right)}$.

Proof In this proof we use the notations of ([17], Section 5.2.4). Recall that $K$ is the kernel of the natural map $T^{\sharp} \rightarrow T$, so $K \widetilde{\Im}\left(\Lambda / \Lambda^{\sharp}\right) \otimes \mu_{n}$. The group $\mathrm{H}^{1}(X, K) \widetilde{\rightarrow} \mathrm{H}^{1}\left(X, \mu_{n}\right) \otimes\left(\Lambda / \Lambda^{\sharp}\right)$ is equipped with the skew-symmetric non-degenerate pairing

$$
(\cdot, \cdot)_{c}: \mathrm{H}^{1}(X, K) \times \mathrm{H}^{1}(X, K) \rightarrow \mu_{n}(k)
$$

described in ([17], Proposition 5.1). Let $H_{0} \subset \mathrm{H}^{1}\left(X, \mu_{n}\right)$ be a maximal isotropic subgroup with respect to the natural pairing $\mathrm{H}^{1}\left(X, \mu_{n}\right) \times \mathrm{H}^{1}\left(X, \mu_{n}\right) \rightarrow \mathrm{H}^{2}\left(X, \mu_{n}^{\otimes 2}\right) \widetilde{\Im} \mu_{n}$. Set $H=$ $H_{0} \otimes\left(\Lambda / \Lambda^{\sharp}\right)$. So, $H \subset \mathrm{H}^{1}(X, K)$ is a $W$-invariant maximal isotropic subgroup with respect to $(\cdot, \cdot)_{c}$.

Recall the stacks ' ${ }^{\prime} \operatorname{Bun}_{T^{\sharp}}, \operatorname{Bun}_{T^{\sharp}, H}$ from ([17], Section 5.2.4). The group $W$ acts naturally on ' $\operatorname{Bun}_{T^{\sharp}}, \operatorname{Bun}_{T}$, and ' $i_{X}:{ }^{\prime} \operatorname{Bun}_{T^{\sharp}} \rightarrow \operatorname{Bun}_{T}$ is $W$-equivariant. The line bundle ' $\tau$ on ${ }^{\prime} \operatorname{Bun}_{T^{\sharp}}$ is naturally $W$-equivariant. The $W$-action on ${ }^{\prime} \mathrm{Bun}_{T^{\sharp}}$ induces a $W$-action on $\operatorname{Bun}_{T^{\sharp}, H}$ so that the diagram ' $\operatorname{Bun}_{T^{\sharp}} \rightarrow \operatorname{Bun}_{T^{\sharp}, H} \rightarrow \operatorname{Bun}_{T}$ is $W$-equivariant. The $W$-actions on 
$\operatorname{Bun}_{T^{\sharp}, H}$ and on $\operatorname{Bun}_{T}$ naturally extend to $W$-actions on $\widetilde{\operatorname{Bun}}_{T^{\sharp}, H}$ and $\widetilde{\operatorname{Bun}_{T}}$. The map $\pi_{H}: \widetilde{\operatorname{Bun}}_{T^{\sharp}, H} \rightarrow \widetilde{\operatorname{Bun}}_{T}$ is $W$-equivariant.

Pick a local system $A E_{H}$ on $\widetilde{\mathrm{Bun}}_{T^{\sharp}, H}$, whose restriction to ${ }^{\prime} \mathrm{Bun}_{T^{\sharp}}$ is identified with $A E$, and such that $\mu_{N}(k)$ acts on it by $\zeta$. Recall that $\mathcal{K}_{E}$ is defined as $\pi_{H !}\left(A E_{H}\right)$. Since the map ${ }^{\prime} \operatorname{Bun}_{T^{\sharp}} \rightarrow \widetilde{\operatorname{Bun}}_{T^{\sharp}, H}$ is $W$-equivariant, our claim follows.

3.6.1. Recall that $\rho_{n}$ denotes the half sum of positive roots of $\check{G}_{n}$. If $w \in W$ then $w\left(\rho_{n}\right)-$ $\rho_{n} \in \Lambda^{\sharp}$. (For $w$ a simple reflection this is clear, the general case is obtained by induction on the length of the decomposition into simple reflections).

Define the twisted $W$-action on $\widetilde{\operatorname{Bun}}_{T}$ as follows. For $\mu \in \Lambda^{\sharp}$ write $\Omega^{\mu}$ for the $T^{\sharp}$-torsor induced from $\Omega$ via $\mu: \mathbb{G}_{m} \rightarrow T^{\sharp}$. By abuse of notation, the corresponding $T$-torsor is also denoted $\Omega^{\mu}$. Denote by $a: \operatorname{Bun}_{T^{\sharp}} \times \widetilde{\operatorname{Bun}}_{T} \rightarrow \widetilde{\operatorname{Bun}}_{T}$ the action of $\operatorname{Bun}_{T^{\sharp}}$ on $\widetilde{\operatorname{Bun}}_{T}$ defined in ([17], Section 5.2.3).

For $\left(\mathcal{F}, \mathcal{U}, \mathcal{U}^{N} \widetilde{\rightarrow} \mathcal{L}_{\mathcal{F}}\right) \in \widetilde{\operatorname{Bun}}_{T}$ we set

$$
w *(\mathcal{F}, \mathcal{U})=a\left(\Omega^{w\left(\rho_{n}\right)-\rho_{n}},\left({ }^{w} \mathcal{F}, \mathcal{U}\right)\right)=\left({ }^{w} \mathcal{F} \otimes \Omega^{w\left(\rho_{n}\right)-\rho_{n}}, \mathcal{U}^{\prime}\right),
$$

where $a$ is the above action map, $\mathfrak{U}^{\prime}$ is the corresponding 1-dimensional space.

Lemma 3.6. The maps (15) define a left $W$-action on $\widetilde{\operatorname{Bun}}_{T}$.

Proof Recall that the line bundles $\mathcal{L}_{T}$ on $\operatorname{Bun}_{T}$ and $\tau$ on $\operatorname{Bun}_{T^{\sharp}}$ are $W$-equivariant.

Let $W$ act as above on $\widetilde{\mathrm{Bun}}_{T}$, and on $\operatorname{Bun}_{T^{\sharp}} \times \widetilde{\mathrm{Bun}}_{T}$ as the product of the $W$-actions on $\operatorname{Bun}_{T^{\sharp}}$ and on $\widetilde{\operatorname{Bun}_{T}}$. Then $a$ is $W$-equivariant.

For $w \in W$ denote by $K \mapsto w * K$ the direct image functor $\mathrm{D}_{\zeta}\left(\widetilde{\operatorname{Bun}_{T}}\right) \rightarrow \mathrm{D}_{\zeta}\left(\widetilde{\operatorname{Bun}_{T}}\right)$ for the new action map $w *: \widetilde{\operatorname{Bun}}_{T} \rightarrow \widetilde{\operatorname{Bun}}_{T}$.

For each simple root $\bar{\alpha}: \mathbb{G}_{m} \rightarrow T^{\sharp}$ of $\check{G}_{n}$ let $a_{\bar{\alpha}}: \operatorname{Bun}_{1} \times \widetilde{\operatorname{Bun}}_{T} \rightarrow \widetilde{\operatorname{Bun}}_{T}$ denote the restriction of $a$ under the push-out map $\operatorname{Bun}_{1} \stackrel{\bar{\alpha}}{\rightarrow} \operatorname{Bun}_{T^{\sharp}}$. Call $\mathcal{S} \in \mathrm{D}_{\zeta}\left(\widetilde{\operatorname{Bun}_{T}}\right)$ regular if for each simple root $\bar{\alpha}$ of $\check{G}_{n}$ one has

$$
\left(a_{\bar{\alpha}}\right) ! \operatorname{pr}_{2}^{*} \mathcal{S}=0
$$

This defines the full triangulated subcategory $\mathrm{D}_{\zeta}\left(\widetilde{\operatorname{Bun}}_{T}\right)^{r e g} \subset \mathrm{D}_{\zeta}\left(\widetilde{\operatorname{Bun}}_{T}\right)$ of regular complexes. Equivalently, instead of (16) one can require the property $\operatorname{pr}_{2 !} a_{\bar{\alpha}}^{*} \mathcal{S}=0$ to define the regularity.

Conjecture 3.1. For $w \in W$ and $\mathcal{S} \in \mathrm{D}_{\zeta}\left(\widetilde{\operatorname{Bun}_{T}}\right)^{\text {reg }}$ there is an isomorphism

$$
\operatorname{Eis}(w * \mathcal{S}) \widetilde{\rightarrow} \operatorname{Eis}(\mathcal{S})
$$

functorial in $\mathcal{S} \in \mathrm{D}_{\zeta}\left(\widetilde{\operatorname{Bun}_{T}}\right)^{\text {reg }}$.

Remark 3.3. Let $E$ be a $\check{T}^{\sharp}$-local system on $X, \mathcal{K}_{E} \in \mathrm{D}_{\zeta}\left(\widetilde{\operatorname{Bun}}_{T}\right)$ be the Hecke eigen-sheaf associated to $E$ in ([17], Proposition 2.2). Then $\mathcal{K}_{E}$ is regular if and only if $E^{\bar{\alpha}}$ is not trivial for each simple root $\bar{\alpha}$ of $\breve{G}_{n}$. 
3.7. Action of $\operatorname{Bun}_{Z(G)}$. The stack $\operatorname{Bun}_{Z(G)}$ is a group stack acting naturally on $\operatorname{Bun}_{G}$ by tensor product. For $\mathcal{T} \in \operatorname{Bun}_{Z(F)}, \mathcal{F} \in \operatorname{Bun}_{G}$ there is a canonical $\mathbb{Z} / 2 \mathbb{Z}$-graded isomorphism

$$
\mathcal{L}_{\mathcal{F} \otimes \mathcal{T}} \widetilde{\sim} \mathcal{L}_{\mathcal{F}}
$$

In particular, $\mathcal{L}$ is canonically trivialized over $\operatorname{Bun}_{Z(G)}$. So, $\operatorname{Bun}_{Z(G)}$ acts on $\widetilde{\operatorname{Bun}}(G$, namely $\mathcal{T} \in \operatorname{Bun}_{Z(G)}$ sends $\left(\mathcal{F}, \mathcal{U}_{G}\right) \in \widetilde{\operatorname{Bun}}_{G}$ to $(\mathcal{F} \otimes \mathcal{T}, \mathcal{U})$.

Set $\left(\Lambda^{\sharp}\right)=\operatorname{Hom}\left(\Lambda^{\sharp}, \mathbb{Z}\right)$. The map $\frac{\iota}{n}: \Lambda \otimes \Lambda^{\sharp} \rightarrow \mathbb{Z}$ yields a map $\frac{\iota}{n}: \Lambda \rightarrow\left(\Lambda^{\sharp}\right)^{\llcorner}$. Consider for a moment $\check{T}^{\sharp}=\mathbb{G}_{m} \otimes\left(\Lambda^{\sharp}\right)^{\check{a}}$ as a torus over Spec $k$. The map $\eta: T \rightarrow \check{T}^{\sharp}$ induced by $\frac{\iota}{n}$ gives the push-out map $\eta_{X}: \operatorname{Bun}_{T} \rightarrow \operatorname{Bun}_{T^{\sharp}}$.

Write $Z\left(\check{G}_{n}\right)_{n}$ for the $n$-torsor subgroup of $Z\left(\check{G}_{n}\right)$.

Lemma 3.7. The map $\eta: T \rightarrow \check{T}^{\sharp}$ sends $Z(G)$ to $Z\left(\check{G}_{n}\right)_{n}$.

Proof For $\bar{\alpha} \in \Lambda^{\sharp}$ one has $\bar{\alpha} \circ \eta=\frac{\iota(\bar{\alpha})}{n}$. If $\bar{\alpha}$ is a simple root of $\check{G}_{n}$ then $\frac{\iota(\bar{\alpha})}{n}$ lies in the roots lattice of $G$. So, for $z \in Z(G)$ we get $\frac{\iota(\bar{\alpha})}{n}(z)=1$, and $\eta(z) \in Z\left(\check{G}_{n}\right)$. To see that $\eta(z) \in Z\left(\check{G}_{n}\right)_{n}$, note that if $\nu \in \Lambda^{\sharp}$ then $\iota(\nu)$ lies in the roots lattice of $G$.

For $\mathcal{T} \in \operatorname{Bun}_{Z(G)}$ let $\mathcal{T}_{\eta}:=\eta_{X}(\mathcal{T})$ denote the corresponding $Z\left(\check{G}_{n}\right)_{n}$-torsor on $X$. For $\nu \in \Lambda^{\sharp}$ denote by $\mathcal{T}_{\eta, \bar{\zeta}}^{\nu}$ the $\overline{\mathbb{Q}}_{\ell}$-local system on $X$ obtained from $\mathcal{T}_{\eta}$ via the push-out by

$$
Z\left(\check{G}_{n}\right)_{n} \stackrel{\nu}{\rightarrow} \mu_{n}(k) \stackrel{\bar{\zeta}}{\rightarrow} \overline{\mathbb{Q}}_{\ell}^{*}
$$

For $\mathcal{T} \in \operatorname{Bun}_{Z(G)}$ denote by $\sigma_{\mathcal{T}}: \widetilde{\operatorname{Bun}}_{G} \rightarrow \widetilde{\operatorname{Bun}}_{G}$ the automorphism $(\mathcal{F}, \mathcal{U}) \mapsto(\mathcal{F} \otimes \mathcal{T}, \mathcal{U})$.

Proposition 3.8. Let $\nu \in \Lambda^{\sharp,+}, \mathcal{T} \in \operatorname{Bun}_{Z(G)}$. The functors $\mathrm{D}_{\zeta}\left(\widetilde{\operatorname{Bun}_{G}}\right) \rightarrow \mathrm{D}_{\zeta}\left(X \times \widetilde{\operatorname{Bun}_{G}}\right)$ given by

$$
K \mapsto \operatorname{pr}_{1}^{*} \mathcal{T}_{\eta, \bar{\zeta}}^{\nu} \otimes \mathrm{H}_{G}^{\nu}\left(\sigma_{\mathcal{T}}^{*} K\right) \quad \text { and by } \quad K \mapsto\left(\mathrm{id} \times \sigma_{\mathcal{T}}\right)^{*} \mathrm{H}_{G}^{\nu}(K)
$$

are naturally isomorphic.

Proof Recall from Section 3.2 that $\overline{\mathcal{H}}_{\tilde{G}}^{\nu}$ classifies $\left(\mathcal{F}, \mathcal{F}^{\prime} \in \operatorname{Bun}_{G}, x \in X, \beta:\left.\mathcal{F} \neg \mathcal{F}^{\prime}\right|_{X-x}, \mathcal{U}, \mathcal{U}^{\prime}\right)$ such that $\mathcal{F}^{\prime}$ is in the position $\leq \nu$ with respect to $\mathcal{F}$ at $x, \mathcal{U}^{N} \widetilde{\neg} \mathcal{L}_{\mathcal{F}}, \mathfrak{U}^{\prime N} \widetilde{\sim} \mathcal{L}_{\mathcal{F}^{\prime}}$.

Let $\operatorname{Bun}_{Z(G)}$ act on $\overline{\mathcal{H}}_{\tilde{G}}^{\nu}$ so that $\mathcal{T} \in \operatorname{Bun}_{Z(G)}$ sends the above point to the collection

$$
\left(\mathcal{F} \otimes \mathcal{T}, \mathcal{F}^{\prime} \otimes \mathcal{T}, x, \beta, \mathcal{U}, \mathcal{U}^{\prime}\right)
$$

with the induced isomorphisms $U^{N} \widetilde{\rightarrow} \mathcal{L}_{\mathcal{F} \otimes \mathcal{T}}, \mathcal{U}^{\prime N} \Im \mathcal{L}_{\mathcal{F}^{\prime} \otimes \mathcal{T}}$. Write $\sigma_{\mathcal{T}}: \overline{\mathcal{H}}_{\tilde{G}}^{\nu} \rightarrow \overline{\mathcal{H}}_{\tilde{G}}^{\nu}$ for this map for a given $\mathcal{T}$. We get a commutative diagram

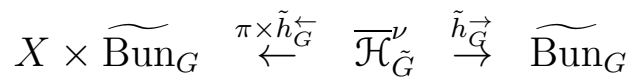

$$
\begin{aligned}
& \downarrow \text { id } \times \sigma_{\mathcal{T}} \quad \downarrow \sigma_{\mathcal{T}} \quad \downarrow \sigma_{\mathcal{T}} \\
& X \times \widetilde{\operatorname{Bun}}_{G} \stackrel{\pi \times \tilde{h}_{\overleftarrow{G}}^{\leftarrow}}{\leftarrow} \overline{\mathcal{H}}_{\tilde{G}}^{\nu} \stackrel{\tilde{h}_{\vec{G}}}{\widetilde{\operatorname{Bun}}_{G}}
\end{aligned}
$$

Note that $\mathcal{H}_{\tilde{G}}^{\nu}$ is preserved by $\sigma_{\mathcal{T}}$. The Hecke functor $\mathrm{H}_{G}^{\nu}$ is defined by formula (6).

Let us establish an isomorphism

$$
\sigma_{\mathcal{T}}^{*} \mathrm{IC}^{\nu} \stackrel{\Im}{\rightarrow} \mathrm{IC}^{\nu} \otimes \pi^{*} \mathcal{T}_{\eta, \bar{\zeta}}^{\nu}
$$


over $\overline{\mathcal{H}}_{\tilde{G}}^{\nu}$. Since both are irreducible perverse sheaves, it suffices to establish it over $\mathcal{H}_{\tilde{G}}^{\nu}$.

As in the proof of Proposition [3.6, there is a line bundle $\mathcal{B}$ on $\mathcal{H}_{G}^{\nu}$ such that $\mathcal{B}^{N}$ is canonically the line bundle with fibre $\mathcal{L}_{\mathcal{F}} \otimes \mathcal{L}_{\mathcal{F}^{\prime}}^{-1}$ at $\left(\mathcal{F}, \mathcal{F}^{\prime}, \beta, x\right) \in \mathcal{H}_{G}^{\nu}$. The line bundle is uniquely defined, as the Picard group is torsion free. We get an isomorphism

$$
\eta: B\left(\mu_{N}\right) \times\left(\mathcal{H}_{G}^{\nu} \times_{\operatorname{Bun}_{G}} \widetilde{\operatorname{Bun}}_{G}\right) \widetilde{\rightarrow} \mathcal{H}_{\tilde{G}}^{\nu}
$$

where we used $h_{G}$ to define the fibred product in parentheses. It sends a collection $(\beta$ : $\left.\left.\mathcal{F} \widetilde{\rightarrow} \mathcal{F}^{\prime}\right|_{X-x}, \mathcal{U}^{\prime N} \widetilde{\rightarrow} \mathcal{L}_{\mathcal{F}^{\prime}}, \mathcal{U}_{0}^{N} \widetilde{\Im} k\right)$ to $\left(\beta, \mathcal{F}, \mathcal{F}^{\prime}, \mathcal{U}, \mathcal{U}^{\prime}\right)$, where $\mathcal{U}=\mathcal{B}_{\left(\mathcal{F}, \mathcal{F}^{\prime}, \beta\right)} \otimes \mathcal{U}_{0} \otimes \mathcal{U}^{\prime}$ with the induced isomorphism $\mathcal{U}^{N} \widetilde{\rightarrow} \mathcal{L}_{\mathcal{F}}$. The perverse sheaf $\mathrm{IC}^{\nu}$ is the intermediate extension of

$$
\eta_{*}\left(\mathcal{L}_{\zeta} \otimes \mathrm{IC}\left(\mathcal{H}_{G}^{\nu} \times_{\mathrm{Bun}_{G}} \widetilde{\operatorname{Bun}}_{G}\right)\right)
$$

Viewing $\mathcal{T}$ as a $T$-torsor on $X$, for $\check{\mu} \in \check{\Lambda}$ we get the line bundle $\mathcal{L}_{\mathcal{T}}^{\check{\mu}}$ on $X$. Over $\mathcal{H}_{G}^{\nu}$ one has an isomorphism

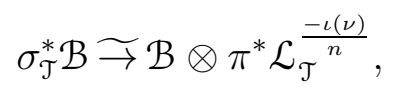

where $\pi: \mathcal{H}_{G}^{\nu} \rightarrow X$ sends $\left(\mathcal{F}, \mathcal{F}^{\prime}, \beta, x\right)$ to $x$. Note that $\mathcal{L}_{\mathcal{T}}^{\frac{\iota(\nu)}{n}}$ is a $\mu_{n}$-torsor on $X$ that we see as a map $X \rightarrow B\left(\mu_{n}\right)$. The restriction of $\mathcal{L}_{\bar{\zeta}}$ under the latter map identifies with $\mathcal{T}_{\eta, \bar{\zeta}}^{\nu}$, because the composition $Z(G) \stackrel{\eta}{\rightarrow} Z\left(\check{G}_{n}\right)_{n} \stackrel{\nu}{\rightarrow} \mu_{n}$ equals $\frac{\iota(\nu)}{n}$. The isomorphism (18) follows. Our claim follows now from the diagram (17).

This applies to Eisenstein series as follows. Recall that a point of $\overline{\mathrm{Bun}}_{\tilde{B}}$ is a collection $\left(\mathcal{F}_{T}, \mathcal{F}, \nu, \mathcal{U}, \mathcal{U}_{G}\right)$, where $\nu^{\check{\lambda}}: \mathcal{L}_{\mathcal{F}_{T}}^{\check{\lambda}} \hookrightarrow \mathcal{V}_{\mathcal{F}}^{\check{\lambda}}$ are inclusions of coherent sheaves for each dominant weight $\check{\lambda}$.

Let $\operatorname{Bun}_{Z(G)}$ act on $\overline{\operatorname{Bun}}_{\tilde{B}}$ so that $\mathcal{T} \in \operatorname{Bun}_{Z(G)} \operatorname{sends}\left(\mathcal{F}_{T}, \mathcal{F}, \nu, \mathcal{U}, \mathcal{U}_{G}\right)$ to $\left(\mathcal{F}_{T} \otimes \mathcal{T}, \mathcal{F} \otimes\right.$ $\left.\mathcal{T}, \nu, \mathcal{U}, \mathcal{U}_{G}\right)$. This action preserves the open substack $\operatorname{Bun}_{\tilde{B}}$. Let $\operatorname{Bun}_{Z(G)}$ act on $\widetilde{\operatorname{Bun}}_{T}$ so that $\mathcal{T}$ sends $\left(\mathcal{F}_{T}, \mathcal{U}\right)$ to $\left(\mathcal{F}_{T} \otimes \mathcal{T}, \mathcal{U}\right)$. The diagram

$$
\widetilde{\operatorname{Bun}}_{T} \stackrel{\tilde{q}}{\leftarrow} \overline{\operatorname{Bun}}_{\tilde{B}} \stackrel{\tilde{\mathfrak{p}}}{\rightarrow} \widetilde{\operatorname{Bun}}_{G}
$$

is $\operatorname{Bun}_{Z(G)}$-equivariant.

Lemma 3.8. Let $K \in \mathrm{D}_{\zeta}\left(\widetilde{\operatorname{Bun}}_{T}\right), \mathcal{T} \in \operatorname{Bun}_{Z(G)}$. One has an isomorphism

$$
\sigma_{\mathcal{T}}^{*} \operatorname{Eis}(K) \stackrel{\sim}{\rightarrow} \operatorname{Eis}\left(\sigma_{\mathcal{T}}^{*} K\right)
$$

functorial in $K \in \mathrm{D}_{\zeta}\left(\widetilde{\operatorname{Bun}}_{T}\right)$.

Proof Write also $\sigma_{\mathcal{T}}: \overline{\operatorname{Bun}}_{\tilde{B}} \rightarrow \overline{\operatorname{Bun}}_{\tilde{B}}$ for the above action map by $\mathcal{T}$. One has $\sigma_{\mathcal{T}}^{*} \mathrm{IC}_{\zeta} \Im \mathrm{IC}_{\zeta}$ canonically. Our claim follows. 


\section{Parabolic Geometric Eisenstein Series}

4.1. Definitions. Let $P \subset G$ be a parabolic containing $B$, let $M$ be its Levi factor, write $\mathcal{J}_{M} \subset \mathcal{J}$ for the corresponding subset. Let $\mathcal{L}_{M}$ denote the restriction of $\mathcal{L}$ under $\operatorname{Bun}_{M} \rightarrow$ $\operatorname{Bun}_{G}$. Let $\widetilde{\operatorname{Bun}}_{M}$ denote the gerb of $N$-th roots of $\mathcal{L}_{M}$.

The notations $\widetilde{\operatorname{Bun}}_{P}, \overline{\operatorname{Bun}}_{P}$ are those of [3]. Let $\widetilde{\operatorname{Bun}}_{P, \tilde{G}}, \overline{\operatorname{Bun}}_{P, \tilde{G}}$ be obtained from $\widetilde{\operatorname{Bun}}_{P}$, $\overline{\operatorname{Bun}}_{P}$ by the base change $\widetilde{\operatorname{Bun}}_{G} \rightarrow \operatorname{Bun}_{G}$. Let $\widetilde{\operatorname{Bun}}_{\tilde{P}}, \overline{\operatorname{Bun}}_{\tilde{P}}$ be obtained from $\widetilde{\operatorname{Bun}}_{P, \tilde{G}}, \overline{\operatorname{Bun}}_{P, \tilde{G}}$ by the base change $\widetilde{\operatorname{Bun}}_{M} \rightarrow \operatorname{Bun}_{M}$.

The Eisenstein series functor Eis $\operatorname{ED}_{M}: \mathrm{D}_{\zeta}\left(\widetilde{\operatorname{Bun}}_{M}\right) \rightarrow \mathrm{D}_{\zeta}\left(\widetilde{\operatorname{Bun}_{G}}\right)$ is defined as follows. By abuse of notations, the diagram of projections is denoted

$$
\widetilde{\operatorname{Bun}}_{M} \stackrel{\tilde{\mathfrak{q}}}{\leftarrow} \widetilde{\operatorname{Bun}}_{\tilde{P}} \stackrel{\tilde{\mathfrak{p}}}{\rightarrow} \widetilde{\operatorname{Bun}}_{G}
$$

A point of $\widetilde{\operatorname{Bun}}_{\tilde{P}}$ is given by a point $\left(\mathcal{F}_{M}, \mathcal{F}, \nu\right) \in \widetilde{\operatorname{Bun}}_{P}$, where

$$
\nu^{\nu}: \mathcal{V}_{\mathcal{F}_{M}}^{U(P)} \hookrightarrow \mathcal{V}_{\mathcal{F}}
$$

is a morphism of coherent sheaves for each representation $\mathcal{V}$ of $G ; \mathcal{U}, \mathcal{U}_{G}$ are $\mathbb{Z} / 2 \mathbb{Z}$-graded lines of parity zero equipped with $\mathcal{U}^{N} \widetilde{\Im}\left(\mathcal{L}_{M}\right)_{\mathcal{F}_{M}}, \mathcal{U}_{G}^{N} \widetilde{\rightrightarrows} \mathcal{L}_{\mathcal{F}}$. The map $\tilde{\mathfrak{q}}$ sends the above point to $\left(\mathcal{F}_{M}, \mathcal{U}\right)$, and $\tilde{\mathfrak{p}}$ sends the above point to $\left(\mathcal{F}, \mathcal{U}_{G}\right)$.

Let $\operatorname{Bun}_{\tilde{P}} \subset \widetilde{\operatorname{Bun}}_{\tilde{P}}$ be the preimage of $\operatorname{Bun}_{P}$ in $\widetilde{\operatorname{Bun}}_{\tilde{P}}$. For a point of $\operatorname{Bun}_{P}$ we have canonically $\left(\mathcal{L}_{M}\right)_{\mathcal{F}_{M}} \stackrel{\widetilde{T}}{\rightarrow} \mathcal{L}_{\mathcal{F}}$. One defines Bun $_{P, \tilde{G}}$ similarly. We get an isomorphism

$$
B\left(\mu_{N}\right) \times \operatorname{Bun}_{P, \tilde{G}} \widetilde{\rightarrow} \operatorname{Bun}_{\tilde{P}}
$$

sending $\left(\mathcal{F}_{P}, \mathcal{U}_{G}, \mathcal{U}_{0} \in B\left(\mu_{N}\right)\right)$ with $\mathcal{U}_{0}^{N} \widetilde{\rightarrow} k$ to $\left(\mathcal{F}_{P}, \mathcal{U}_{G}, \mathcal{U}\right)$ with $\mathcal{U}=\mathcal{U}_{G} \otimes \mathcal{U}_{0}^{-1}$. By definition,

$$
\tilde{\mathfrak{q}}\left(\mathcal{F}_{M}, \mathcal{F}, \nu, \mathcal{U}, \mathcal{U}_{G}\right)=\left(\mathcal{F}_{M}, \mathcal{U}\right) \text { and } \tilde{\mathfrak{p}}\left(\mathcal{F}_{M}, \mathcal{F}, \nu, \mathcal{U}, \mathcal{U}_{G}\right)=\left(\mathcal{F}, \mathcal{U}_{G}\right)
$$

View $\mathcal{L}_{\zeta} \otimes \mathrm{IC}\left(\operatorname{Bun}_{P, \tilde{G}}\right)$ as a perverse sheaf on $\operatorname{Bun}_{\tilde{P}}$ via (20). We still denote by $\mathrm{IC}_{\zeta}$ the intermediate extension of this perverse sheaf to $\widetilde{\operatorname{Bun}}_{\tilde{P}}$. Write $j_{\tilde{P}}: \operatorname{Bun}_{\tilde{P}} \hookrightarrow \widetilde{\operatorname{Bun}}_{\tilde{P}}$ for the natural open immersion.

Definition 4.1. For $K \in \mathrm{D}_{\zeta}\left(\widetilde{\operatorname{Bun}}_{M}\right)$ set

$$
\operatorname{Eis}_{M}(K)=\tilde{\mathfrak{p}}_{!}\left(\tilde{\mathfrak{q}}^{*} K \otimes \mathrm{IC}_{\zeta}\right)\left[-\operatorname{dim} \operatorname{Bun}_{M}\right]
$$

This gives a functor $\mathrm{D}_{\zeta}\left(\widetilde{\operatorname{Bun}}_{M}\right) \rightarrow \mathrm{D}_{\zeta}\left(\widetilde{\operatorname{Bun}}_{G}\right)$.

4.1.1. Set $\Lambda_{M, 0}=\left\{\lambda \in \Lambda \mid\left\langle\lambda, \check{\alpha}_{i}\right\rangle=0\right.$ for all $\left.i \in \mathcal{J}_{M}\right\}$. Let $Z(M)^{0}=\mathbb{G}_{m} \otimes \Lambda_{M, 0}$, this is the connected component of unity of the center of $M$. Denote by $\check{\Lambda}_{M, 0}$ the lattice dual to $\Lambda_{M, 0}$. To $\mu \in \Lambda_{G, P}$ we associate the character $\Lambda_{M, 0} \rightarrow \mathbb{Z}, \lambda \mapsto \kappa(\lambda, \mu)$ denoted $\kappa_{M}(\mu)$. This is well-defined, because $\kappa\left(\alpha_{i}\right) \in \mathbb{Z} \check{\alpha}_{i}$, and gives a homomorphism $\kappa_{M}: \Lambda_{G, P} \rightarrow \check{\Lambda}_{M, 0}$.

The group $Z(M)^{0}$ acts on $\operatorname{Bun}_{M}$ by 2-automorphisms naturally. As in Section 2.0 .4 for $T$ one checks the following. If $\theta \in \Lambda_{G, P}, \mathcal{F} \in \operatorname{Bun}_{M}^{\theta}$ then $Z(M)^{0}$ acts on $\left(\mathcal{L}_{M}\right)_{\mathcal{F}}$ by the character $-\kappa_{M}(\theta)$. The following is a generalization of ([17], Proposition 2.1). 
Proof. Proof of Proposition 2.1 A $k$-point $\mathcal{F} \in \operatorname{Bun}_{M}^{\theta}$ defines a map $f: B\left(Z(M)^{0}\right) \rightarrow \operatorname{Bun}_{M}^{\theta}$. Let $\widetilde{B}\left(Z(M)^{0}\right)$ be the restriction of the gerb $\widetilde{\operatorname{Bun}}_{M}^{\theta} \rightarrow \operatorname{Bun}_{M}^{\theta}$ under this map. As above, we get the category $\mathrm{D}_{\zeta}\left(\widetilde{B}\left(Z(M)^{0}\right)\right)$. By $\left([17]\right.$, Lemma 5.3), $\mathrm{D}_{\zeta}\left(\widetilde{B}\left(Z(M)^{0}\right)\right)=0$ unless $\kappa_{M}(\theta) \in N \check{\Lambda}_{M, 0}$. Our claim follows.

Proposition 4.1. The complexes $\mathrm{IC}_{\zeta}$ and $\left(j_{\tilde{P}}\right) ! j_{\tilde{P}}^{*} \mathrm{IC}_{\zeta}$ are $U L A$ with respect to $\tilde{\mathfrak{q}}: \widetilde{\operatorname{Bun}}_{\tilde{P}} \rightarrow$ $\widetilde{\operatorname{Bun}}_{M}$.

4.1.2. Proof of Proposition 4.1. The argument from (3], Proposition 5.1.5) applies in our setting. For $\mathcal{F}_{M}, \mathcal{F}_{M}^{\prime} \in \operatorname{Bun}_{M}(k)$ write $\mathcal{F}_{M} \prec \mathcal{F}_{M}^{\prime}$ if there is $\lambda \in \Lambda^{+} \operatorname{such}$ that $\left(\mathcal{F}_{M}, \mathcal{F}_{M}^{\prime}\right)$ is the image of

$$
\mathcal{H}_{M}^{\lambda} \stackrel{h \stackrel{\leftrightarrow}{M} \times h_{M}^{\rightarrow}}{\rightarrow} \operatorname{Bun}_{M} \times \operatorname{Bun}_{M}
$$

of some $k$-point. Let $\sim$ be the equivalence relation on $\operatorname{Bun}_{M}(k)$ generated by $\prec$. Write $\Lambda_{G, P}^{\sharp}$ for the image of the natural map $\Lambda^{\sharp} \rightarrow \Lambda_{G, P}$.

Let $\Lambda_{G, P}^{\text {pos }}$ be the $\mathbb{Z}_{+}$-span of $\alpha_{i}, i \in \mathcal{J}-\mathcal{J}_{M}$ in $\Lambda_{G, P}$. For $\theta \in \Lambda_{G, P}^{\text {pos }}$ we have the open substack $\widetilde{\operatorname{Bun}}_{P}^{\leq \theta}$ defined in ([3], Section 5.3.1). Recall that $\cup_{\theta} \widetilde{\operatorname{Bun}}_{P}^{\leq \theta}=\widetilde{\operatorname{Bun}}_{P}$, so it is sufficient to show that $\mathrm{IC}_{\zeta}$ is ULA with respect to $\widetilde{\operatorname{Bun}_{\tilde{P}}} \rightarrow \theta \widetilde{\operatorname{Bun}}_{M}$ for any $\theta \in \Lambda_{G, P}^{\theta}$.

Let $\stackrel{\circ}{\operatorname{Bun}}_{M}$ denote the biggest open substack of $\operatorname{Bun}_{M}$ such that for its preimage $\stackrel{\circ}{\operatorname{Bun}_{M}}$ in

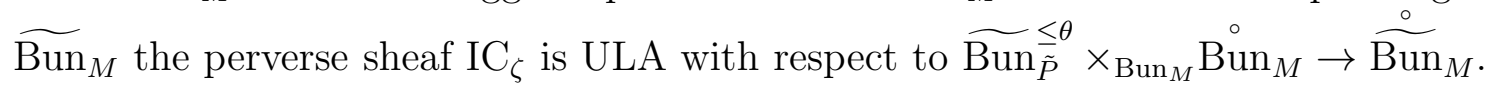

Proposition 4.2. If $\mathcal{F}_{M} \prec \mathcal{F}_{M}$ are k-points of $\operatorname{Bun}_{M}$ then $\mathcal{F}_{M}^{\prime} \in \operatorname{Bun}_{M}$ if and only if $\mathcal{F}_{M} \in \operatorname{Bun}_{M}$.

Proof. The argument from ([3], Proposition 5.3.4) applies. One only needs to check the following. Pick $\lambda \in \Lambda^{+}$. Consider the stack $Z$ classifying

$$
\left(x \in X, \mathcal{F}_{G}, \mathcal{F}_{M}, \mathcal{F}_{G}^{\prime}, \mathcal{F}_{M}^{\prime}, \kappa, \kappa^{\prime}, \beta, \beta_{M}, \mathcal{U}, \mathcal{U}^{\prime}, \mathcal{U}_{G}, \mathcal{U}_{G}^{\prime}\right)
$$

where $\mathcal{U}, \mathcal{U}^{\prime}, \mathcal{U}_{G}, \mathcal{U}_{G}^{\prime}$ are lines equipped with isomorphisms

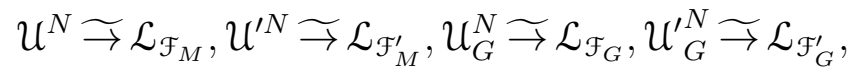

$$
\begin{aligned}
& \left(\mathcal{F}_{G}, \mathcal{F}_{M}, \kappa, \mathcal{U}, \mathcal{U}_{G}\right) \in \widetilde{\operatorname{Bun}_{\tilde{P}}},\left(\mathcal{F}_{G}^{\prime}, \mathcal{F}_{M}^{\prime}, \kappa^{\prime}, \mathcal{U}^{\prime}, \mathcal{U}_{G}^{\prime}\right) \in \widetilde{\operatorname{Bun}_{\tilde{P}}} \text {, and } \\
& \beta:\left.\mathcal{F}_{G} \widetilde{\rightarrow} \mathcal{F}_{G}^{\prime}\right|_{X-x}, \beta_{M}:\left.\mathcal{F}_{M} \widetilde{\rightarrow} \mathcal{F}_{M}^{\prime}\right|_{X-x}
\end{aligned}
$$

such that

- $\mathcal{F}_{G}^{\prime}$ is in the $G$-position $\lambda$ with respect to $\mathcal{F}_{G}$ at $x$;

- $\mathcal{F}_{M}^{\prime}$ is in the $M$-position $\lambda$ with respect to $\mathcal{F}_{M}$ at $x$;

- the maps $\kappa: V_{\mathcal{F}_{M}}^{U(P)} \rightarrow V_{\mathcal{F}_{G}}$ have no zero at $x$ for any $V \in \operatorname{Rep}(G)$;

- the maps $\kappa^{\prime}: V_{\mathcal{F}_{M}^{\prime}}^{U(P)} \rightarrow V_{\mathcal{F}_{G}^{\prime}}$ have no zero at $x$ for any $V \in \operatorname{Rep}(G)$. 
We have two smooth projections $\widetilde{\operatorname{Bun}}_{\tilde{P}} \stackrel{h \leftarrow}{\leftarrow} Z \stackrel{h \rightarrow}{\rightarrow} \widetilde{\operatorname{Bun}}_{\tilde{P}}$, where $h^{\leftarrow}$ and $h \rightarrow$ sends the above point to

$$
\left(\mathcal{F}_{G}, \mathcal{F}_{M}, \kappa, \mathcal{U}, \mathcal{U}_{G}\right) \text { and }\left(\mathcal{F}_{G}^{\prime}, \mathcal{F}_{M}^{\prime}, \kappa^{\prime}, \mathcal{U}^{\prime}, \mathcal{U}_{G}^{\prime}\right)
$$

respectively. Then the line bundle on $Z$ with fibre $\mathcal{L}_{\mathcal{F}_{G}} \otimes \mathcal{L}_{\mathcal{F}_{M}^{\prime}} \otimes \mathcal{L}_{\mathcal{F}_{M}}^{*} \otimes \mathcal{L}_{\mathcal{F}_{G}^{\prime}}^{*}$ is canonically trivialized. So, the perverse sheaves $\left(h^{\rightarrow}\right)^{*} \mathrm{IC}_{\zeta}\left[\operatorname{dim} \cdot \operatorname{rel}\left(h^{\rightarrow}\right)\right]$ and $\left(h^{\leftarrow}\right)^{*} \mathrm{IC}_{\zeta}\left[\operatorname{dim} \cdot \operatorname{rel}\left(h^{\leftarrow}\right)\right]$ are locally isomorphic in the smooth topology on $Z$. The rest of the argument is exactly as in ([3], Proposition 5.3.4).

One finishes the proof of Proposition 4.1 now as in ([3], Theorem 5.1.5). Theorem 2.3 also follows from Proposition 4.1 as in the case $M=T$.

4.2. Hecke functors for $M$. Let $\mathcal{L}$ also denote the restriction of $\mathcal{L}$ under $\operatorname{Gr}_{M} \rightarrow \operatorname{Gr}_{G}$. Let $\widetilde{\mathrm{Gr}}_{M} \rightarrow \widetilde{\mathrm{Gr}}_{P}$ be obtained from $\mathrm{Gr}_{M} \rightarrow \mathrm{Gr}_{P}$ by the base change $\widetilde{\mathrm{Gr}}_{G} \rightarrow \mathrm{Gr}_{G}$. Let $\operatorname{Perv}_{M, G, n}$ be the category of $M(\mathbf{O})$-equivariant perverse sheaves on $\widetilde{G r}_{M}$, on which $\mu_{N}(k)$ acts by $\zeta$.

Let $\Lambda_{M}^{+} \subset \Lambda$ be the coweights of $T$ dominant for $M$. Set $\Lambda_{M}^{\sharp,+}=\Lambda^{\sharp} \cap \Lambda_{M}^{+}$. As in ([9], Section 4.1.1) for $\nu \in \Lambda_{M}^{\sharp,+}$ we get the perverse sheaf $\mathcal{A}_{M, \mathcal{E}}^{\nu} \in \operatorname{Perv}_{M, G, n}$ on $\widetilde{\mathrm{Gr}}_{M}$. Here $\mathcal{E}$ is the square root of $\Omega(\mathbf{O})$ that we picked in Section 3.2 .

Recall that $\check{M}_{n} \subset \breve{G}_{n}$ is the standard Levi subgroup corresponding to $\mathcal{J}_{M}$. Note that $\Lambda_{M}^{\sharp++}$ are exactly the dominant weights of $\check{M}_{n}$. In ([9], Section 4.2) we introduced a tensor category $\operatorname{Perv}_{M, G, n}^{\natural}$ (obtained from $\mathbb{P e r v}_{M, G, n}$ by changing the commutativity constraint)

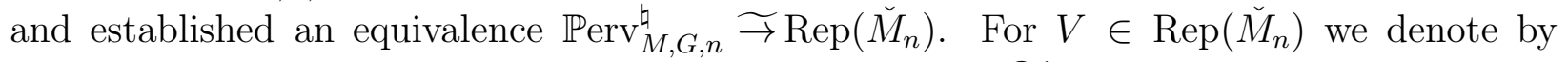
$\operatorname{Loc}(V) \in \operatorname{Perv}_{M, G, n}$ the corresponding perverse sheaf on $\widetilde{G r}_{M}$. If $V$ is an irreducible $\check{M}$ module with highest weight $\nu$ then $\operatorname{Loc}(V)=\mathcal{A}_{M, \mathcal{\varepsilon}}^{\nu}$. We write $\operatorname{Loc}_{\zeta}(V)$ if we need to express the dependence on $\zeta$.

The Hecke stack $\mathcal{H}_{M}$, the diagram

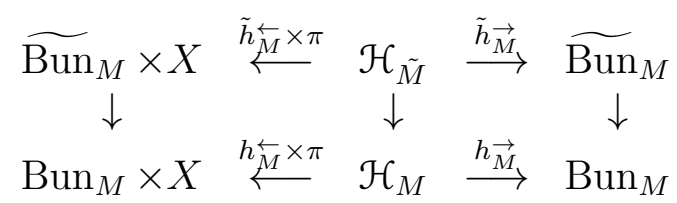

and $\operatorname{Gr}_{M, X}$ are defined as for $G$. The stack $\mathcal{H}_{\tilde{M}}$ classifies $M$-torsors $\mathcal{F}_{M}, \mathcal{F}_{M}^{\prime}$ on $X, x \in$ $X, \beta_{M}:\left.\mathcal{F}_{M} \widetilde{\rightarrow} \mathcal{F}_{M}^{\prime}\right|_{X-x}$ together with lines $\mathcal{U}, \mathcal{U}^{\prime}$ equipped with

$$
\mathcal{U}^{N} \widetilde{\rightarrow}\left(\mathcal{L}_{M}\right)_{\mathcal{F}_{M}}, \quad \mathcal{U}^{\prime N} \widetilde{\rightarrow}\left(\mathcal{L}_{M}\right)_{\mathcal{F}_{M}^{\prime}}
$$

The line bundle $\mathcal{L}_{X}$ on $\operatorname{Gr}_{M, X}$ is the restriction of $\mathcal{L}_{X}$ under $\operatorname{Gr}_{M, X} \rightarrow \operatorname{Gr}_{G, X}$. We similarly have the isomorphisms $\gamma^{\leftarrow}$ (resp., $\gamma^{\rightarrow}$ )

$$
\operatorname{Bun}_{M, X} \times_{M_{X}} \operatorname{Gr}_{M, X} \widetilde{\rightarrow} \mathcal{H}_{M}
$$

such that the projection to the first term corresponds to $h_{M}^{\overleftarrow{M}}$ (resp., $h_{M}$ ). Over the stack $\operatorname{Bun}_{M, X} \times_{M_{X}} \operatorname{Gr}_{M, X}$ we have canonically the isomorphism

$$
\left(\gamma^{\rightarrow}\right)^{*}\left(h_{M}^{\overleftarrow{M}}\right)^{*} \mathcal{L}_{M} \widetilde{\rightrightarrows} \mathcal{L}_{M} \tilde{\otimes} \mathcal{L}_{X}
$$


Let $\operatorname{Perv}_{M, G, n, X}$ be the category of $K \in \mathrm{D}\left(\widetilde{\mathrm{Gr}}_{M, X}\right)$ such that $K[1]$ is perverse, $M_{X^{-}}$ equivariant, and $\mu_{N}(k)$ acts on $K$ by $\zeta$. We have the fully faithful functor defined in ([9], Section 4.1.2)

$$
\tau^{0}: \mathbb{P e r v}_{M, G, n} \rightarrow \operatorname{Perv}_{M, G, n, X}
$$

For $\nu \in \Lambda_{M}^{\sharp,+}$ set $\mathcal{A}_{M}^{\nu}=\tau^{0}\left(\mathcal{A}_{M, \varepsilon}^{\nu}\right) \in \operatorname{Perv}_{M, G, n, X}$.

Consider the $M_{X}$-torsor

$$
\tilde{\gamma} \rightarrow: \widetilde{\operatorname{Bun}}_{M, X} \times{ }_{X} \widetilde{\operatorname{Gr}}_{M, X} \rightarrow \mathcal{H}_{\tilde{M}}
$$

For a $M_{X}$-equivariant perverse sheaf $\mathcal{S}$ on $\widetilde{\mathrm{Gr}}_{M, X}$ and $\mathcal{T} \in \mathrm{D}\left(\widetilde{\operatorname{Bun}}_{M}\right)$ one defines their twisted exterior product $(\mathcal{T} \tilde{\otimes} \mathcal{S})^{r}$ on $\mathcal{H}_{\tilde{M}}$. This is the descent via $\tilde{\gamma} \rightarrow$, it is normalized to be perverse for $\mathcal{T}, \mathcal{S}$ perverse. Similarly, one gets $(\mathcal{T} \tilde{\otimes} \mathcal{S})^{l}$ on $\mathcal{H}_{\tilde{M}}$.

Now for $\nu \in \Lambda_{M}^{\sharp,+}$ let

$$
\mathrm{H}_{M}^{\nu}: \mathrm{D}_{\zeta}\left(\widetilde{\operatorname{Bun}}_{M}\right) \rightarrow \mathrm{D}_{\zeta}\left(\widetilde{\operatorname{Bun}}_{M} \times X\right)
$$

be given by

$$
\mathrm{H}_{M}^{\nu}(\mathcal{T})=\left(\tilde{h}_{M}^{\overleftarrow{ }} \times \pi\right)_{!}\left(\left(\mathcal{T} \tilde{\otimes} \mathcal{A}_{M}^{-w_{0}^{M}(\nu)}\right)^{r}\right)
$$

As for $G$, one has the covariant functor $\star: \operatorname{Perv}_{M, G, n, \zeta^{-1}} \rightarrow \operatorname{Perv}_{M, G, n, \zeta}$. For $\nu \in \Lambda_{M}^{\sharp,+}$ it sends $\mathcal{A}_{M, \mathcal{E}}^{\nu}$ to $\mathcal{A}_{M, \mathcal{E}}^{-w_{0}^{M}(\nu)}$. For $\mathcal{S} \in \mathbb{P e r v}_{M, G, n}, \mathcal{T} \in \mathrm{D}_{\zeta}\left(\widetilde{\operatorname{Bun}}_{M}\right)$ we define

$$
\mathrm{H}_{M}^{\leftarrow}(\mathcal{S}, \mathcal{T})=\left(\tilde{h}_{M}^{\overleftarrow{M}} \times \pi\right)_{!}\left(\mathcal{T} \tilde{\otimes} \tau^{0}(\star \mathcal{S})\right)^{r} \quad \text { and } \quad \mathrm{H}_{M}^{\rightarrow}(\mathcal{S}, \mathcal{T})=\left(\tilde{h}_{M} \times \pi\right)_{!}\left(\mathcal{F} \tilde{\otimes} \tau^{0}(\mathcal{S})\right)^{l}
$$

4.3. Geometric restriction functors. Write $\Lambda_{G, P}=\Lambda /\left\{\alpha_{i}, i \in \mathcal{J}_{M}\right\}$ for the lattice of cocharacters of $M /[M, M]$. Let $\check{\Lambda}_{G, P}$ denote the dual lattice. For $\theta \in \Lambda_{G, P}$ the connected component $\operatorname{Gr}_{M}^{\theta}$ is the one containing $t^{\nu} M(\mathbf{O})$ for any $\nu \in \Lambda$ over $\theta$. For $\theta \in \Lambda_{G, P}$ denote by $\operatorname{Gr}_{P}^{\theta}$ the ind-scheme classifying $\left(\mathcal{F}_{G}, \beta:\left.\mathcal{F}_{G} \widetilde{\rightarrow} \mathcal{F}_{G}^{0}\right|_{D_{x}^{*}}\right) \operatorname{Gr}_{G}$ such that for any $\check{\lambda} \in \check{\Lambda}^{+} \cap \check{\Lambda}_{G, P}$ the corresponding map

$$
\kappa^{\check{\lambda}}: \mathcal{L}_{\mathcal{F}_{T}^{0}}^{\check{\lambda}}(-\langle\theta, \check{\lambda}\rangle x) \rightarrow \mathcal{V}_{\mathcal{F}_{G}}^{\check{\lambda}}
$$

is regular and has no zero. This ind-scheme was denoted $S_{P}^{\theta}$ in ([3], Section 4.3.1).

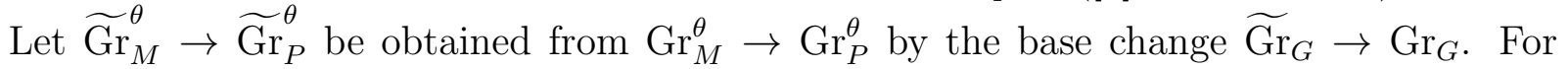
$\theta \in \Lambda_{G, P}$ we have the diagram

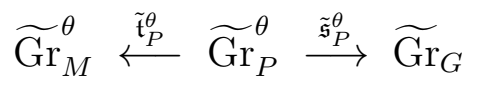

The next result follows from [9].

Proposition 4.3. There is a functor gRes : $\mathbb{P e r v}_{G, n}^{\natural} \rightarrow \mathbb{P e r v}_{M, G, n}^{\natural}$ given by

$$
K \mapsto \underset{\theta \in \Lambda_{G, P}}{\oplus}\left(\tilde{\mathfrak{t}}_{P}^{\theta}\right)_{!}\left(\tilde{\mathfrak{s}}_{P}^{\theta}\right)^{*} K\left[\left\langle\theta, 2 \check{\rho}-2 \check{\rho}_{M}\right\rangle\right],
$$


and the following diagram of categories naturally commutes

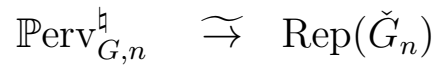

$$
\begin{aligned}
& \downarrow \text { gRes } \downarrow \text { Res }
\end{aligned}
$$

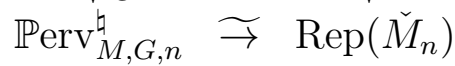

Here the horizontal equivalences are those constructed in [9], and Res is the restriction functor for $\check{M}_{n} \hookrightarrow \check{G}_{n}$.

Let $\Lambda_{G, P}^{\sharp}$ denote the image of the map $\Lambda^{\sharp} \rightarrow \Lambda_{G, P}$ given by $\mu \mapsto \mu$. Note that $\Lambda_{G, P}^{\sharp}$ is a subgroup of finite index in the free abelian group $\Lambda_{G, P}$, so $\Lambda_{G, P}^{\sharp}$ is also free. In the formula (21) for gRes one may replace $\Lambda_{G, P}$ by $\Lambda_{G, P}^{\sharp}$.

The center $Z\left(\check{M}_{n}\right)$ is not connected in general under our assumptions. Write $C^{*}\left(\check{M}_{n}\right)$ for the cocenter of $\check{M}_{n}$, that is, the quotient of $\Lambda^{\sharp}$ by the roots lattice of $\check{M}_{n}$. We have canonically $\operatorname{Hom}\left(Z\left(\check{M}_{n}\right), \mathbb{G}_{m}\right) \stackrel{\rightarrow}{\rightarrow} C^{*}\left(\check{M}_{n}\right)$ by $\left([27,2.15(\mathrm{~b}))\right.$. The natural map $c_{P}: C^{*}\left(\check{M}_{n}\right) \rightarrow$ $\Lambda_{G, P}^{\sharp}$ is surjective, but not injective in general. Its kernel is finite and coincides with the torsion subgroup in $C^{*}\left(\check{M}_{n}\right)$. Indeed, if $\sum_{i} a_{i} \alpha_{i} \in \Lambda$ vanishes in $\Lambda_{G, P}$ then it is of the form $\sum_{i \in \mathcal{J}_{M}} a_{i} \alpha_{i}$, and a multiple of this element lies in the roots lattice $\oplus_{i \in \mathcal{J}_{M}} \delta_{i} \alpha_{i} \mathbb{Z}$ of $\check{M}_{n}$.

Recall that for $\nu \in \Lambda_{M}^{\sharp,+}$ we denote by $U^{\nu}$ the irreducible representation of $\check{M}_{n}$ with highest weight $\nu$.

For $\nu \in \Lambda_{M}^{+}$lying over $\theta \in \Lambda_{G, P}$ let $\tilde{\mathfrak{t}}_{P}^{\nu}:{\widetilde{\mathrm{Gr}_{P}}}^{\nu} \rightarrow \widetilde{\mathrm{Gr}}_{M}^{\nu}$ be the map obtained from $\tilde{\mathfrak{t}}_{P}^{\theta}$ by the base change $\widetilde{\mathrm{Gr}}_{M}^{\nu} \rightarrow{\widetilde{\mathrm{Gr}_{M}}}_{M}^{\theta}$. For $\mu \in \Lambda^{\sharp,+}$ recall the local system $E_{\mathcal{\varepsilon}}^{\mu}$ over $\widetilde{\mathrm{Gr}}_{G}^{\mu}$. From Proposition 4.3 one gets the following.

Corollary 4.1. Let $\nu \in \Lambda_{M}^{+}$lying over $\theta \in \Lambda_{G, P}$. Let $\mu \in \Lambda^{\sharp,+}$. The complex

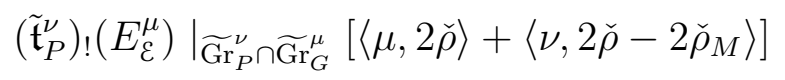

is placed in perverse degrees $\leq 0$, its 0 -th perverse cohomology sheaf vanishes unless $\nu \in$ $\Lambda_{M}^{\sharp,+}$, and in the latter case identifies canonically with $\mathcal{A}_{M, \varepsilon}^{\nu} \otimes \operatorname{Hom}_{\check{M}_{n}}\left(U^{\nu}, V^{\mu}\right)$. The space $\operatorname{Hom}_{\check{M}_{n}}\left(U^{\nu}, V^{\mu}\right)$ admits a base formed by those connected components $C$ of $\widetilde{\mathrm{Gr}}_{P}^{\nu} \cap \widetilde{\mathrm{Gr}}_{G}^{\mu}$ of dimension $\langle\mu+\nu, \check{\rho}\rangle$ for which $E_{\mathcal{\varepsilon}}^{\mu}$ descends under the map $\tilde{\mathfrak{t}}_{P}^{\nu}: C \rightarrow \widetilde{\mathrm{Gr}}_{M}^{\nu}$.

Note that the descent property in Corollary 4.1 can be checked at the generic point of the component $C$.

4.4. Proof of Theorem 2.4. The proof of ([3], Theorem 2.3.7) essentially applies in our setting with some minor changes that we explain. To simplify notations, we prove a version of Theorem 2.4 with $x \in X$ fixed.

4.4.1. We have the stack ${ }_{x, \infty} \widetilde{\operatorname{Bun}}_{P}$ defined in ([3], Section 4.1.1). For $\nu \in \Lambda_{M}^{+}$one also has the diagram ${ }_{x, \nu} \widetilde{\operatorname{Bun}}_{P} \hookrightarrow{ }_{x, \geq \nu} \widetilde{\operatorname{Bun}}_{P} \hookrightarrow{ }_{x, \infty} \widetilde{\operatorname{Bun}}_{P}$ defined in ([3], Sections 4.1.1 and 4.2.1), the first map is an open immersion, the second one is a closed immersion. Let ${ }_{x, \nu} \widetilde{\operatorname{Bun}}_{\tilde{P}} \hookrightarrow$ 
${ }_{x, \geq \nu} \widetilde{\operatorname{Bun}}_{\tilde{P}} \hookrightarrow{ }_{x, \infty} \widetilde{\operatorname{Bun}_{\tilde{P}}}$ be obtained from the above by the base change $\widetilde{\operatorname{Bun}}_{M} \times \widetilde{\operatorname{Bun}}_{G} \rightarrow$ $\operatorname{Bun}_{M} \times \operatorname{Bun}_{G}$.

Recall the stacks ${ }_{x, \infty} Z_{P, M}$ and ${ }_{x, \infty} Z_{P, G}$ defined in ([3], Section 4.1.2 and 4.1.4). We similarly define the stacks ${ }_{x, \infty} Z_{\tilde{P}, \tilde{M}}$ and ${ }_{x, \infty} Z_{\tilde{P}, \tilde{G}}$ included into the diagrams

$$
\begin{aligned}
& \widetilde{\operatorname{Bun}}_{\tilde{P}} \stackrel{\prime \tilde{h}_{M}^{\overleftarrow{M}}}{\leftarrow}{ }_{x, \infty} Z_{\tilde{P}, \tilde{M}} \stackrel{{ }^{\prime} \tilde{h}_{M}}{\rightarrow} \quad \widetilde{\operatorname{Bun}}_{\tilde{P}} \\
& \downarrow \tilde{\mathfrak{q}}_{P} \quad \downarrow \tilde{\mathfrak{q}}^{\prime} \tilde{\mathfrak{q}}_{P} \quad \downarrow \tilde{\mathfrak{q}}_{P} \\
& \widetilde{\operatorname{Bun}}_{M} \stackrel{\tilde{h}_{M}^{\leftarrow}}{\stackrel{\leftarrow}{\leftrightarrows}}{ }_{x} \mathcal{H}_{\tilde{M}} \quad \stackrel{\tilde{h}_{M}}{\rightarrow} \quad \widetilde{\operatorname{Bun}}_{M}
\end{aligned}
$$

and

$$
\begin{aligned}
& \widetilde{\operatorname{Bun}}_{\tilde{P}} \stackrel{\prime \tilde{h}_{G}^{\overleftarrow{G}}}{\leftarrow}{ }_{x, \infty} Z_{\tilde{P}, \tilde{G}} \stackrel{{ }^{\prime} \tilde{h}_{G}}{\rightarrow} \quad \widetilde{\operatorname{Bun}}_{\tilde{P}} \\
& \downarrow \tilde{\mathfrak{p}}_{P} \quad \downarrow \tilde{\mathfrak{p}}_{P} \quad \downarrow \tilde{\mathfrak{p}}_{P} \\
& \widetilde{\operatorname{Bun}}_{G} \quad \tilde{h}_{G}^{\leftarrow} \quad{ }_{x} \mathcal{H}_{\tilde{G}} \quad \stackrel{\tilde{h}_{G}^{\rightarrow}}{\widetilde{\operatorname{Bun}}_{G}}
\end{aligned}
$$

Both squares in each of the above diagrams are cartesian.

A point of ${ }_{x, \infty} \widetilde{\operatorname{Bun}}_{\tilde{P}}$ is $\left(\mathcal{F}_{M}, \mathcal{F}_{G}, \kappa\right) \in{ }_{x, \infty} \widetilde{\operatorname{Bun}}_{P}$ and $\left(\mathcal{F}_{M}, \mathcal{U}\right) \in \widetilde{\operatorname{Bun}}_{M},\left(\mathcal{F}_{G}, \mathcal{U}_{G}\right) \in \widetilde{\operatorname{Bun}}_{G}$. Let $\mu_{N}(k) \times \mu_{N}(k)$ act on ${ }_{x, \infty} \widetilde{\operatorname{Bun}}_{\tilde{P}}$ by 2 -automorphisms so that $\left(a, a_{G}\right)$ acts as $a$ on $\mathcal{U}$, as $a_{G}$ on $\mathcal{U}_{G}$ and trivially on $\left(\mathcal{F}_{M}, \mathcal{F}_{G}, \kappa\right)$. Denote by

$$
\mathrm{D}_{\zeta}\left({ }_{x, \infty} \widetilde{\operatorname{Bun}}_{\tilde{P}}\right) \subset \mathrm{D}\left({ }_{x, \infty} \widetilde{\operatorname{Bun}}_{\tilde{P}}\right)
$$

the full subcategory of objects on which any $\left(a, a_{G}\right) \in \mu_{N}(k) \times \mu_{N}(k)$ acts by $\zeta\left(\frac{a_{G}}{a}\right)$.

Now for $\mathcal{S} \in \operatorname{Perv}_{M, G, n}, \mathcal{T} \in \mathrm{D}_{\zeta}\left({ }_{x, \infty} \widetilde{\operatorname{Bun}}_{\tilde{P}}\right)$ one defines the functors

$$
{ }_{x} \mathrm{H}_{P, M}^{\leftarrow},{ }_{x} \mathrm{H}_{P, M}^{\rightarrow}: \operatorname{Perv}_{M, G, n} \times \mathrm{D}_{\zeta}\left({ }_{x, \infty} \widetilde{\operatorname{Bun}}_{\tilde{P}}\right) \rightarrow \mathrm{D}_{\zeta}\left({ }_{x, \infty} \widetilde{\operatorname{Bun}}_{\tilde{P}}\right)
$$

and

$$
{ }_{x} \mathrm{H}_{P, G}^{\leftarrow},{ }_{x} \mathrm{H}_{P, G}: \operatorname{Perv}_{G, n} \times \mathrm{D}_{\zeta}\left({ }_{x, \infty} \widetilde{\operatorname{Bun}_{\tilde{P}}}\right) \rightarrow \mathrm{D}_{\zeta}\left({ }_{x, \infty} \widetilde{\operatorname{Bun}}_{\tilde{P}}\right)
$$

as in ([3], Sections 4.1.2-4.1.4). In particular, for $\nu \in \Lambda_{M}^{\sharp,+}$ we get

$$
{ }_{x} \mathrm{H}_{P, M}^{\nu}: \mathrm{D}_{\zeta}\left({ }_{x, \infty} \widetilde{\operatorname{Bun}}_{\tilde{P}}\right) \rightarrow \mathrm{D}_{\zeta}\left({ }_{x, \infty} \widetilde{\operatorname{Bun}}_{\tilde{P}}\right)
$$

given by ${ }_{x} \mathrm{H}_{P, M}^{\nu}(\mathcal{T})={ }_{x} \mathrm{H}_{P, M}^{\leftarrow}\left(\mathcal{A}_{\varepsilon}^{\nu}, \mathcal{T}\right)$. For $\lambda \in \Lambda^{\sharp,+}$ we get

$$
{ }_{x} \mathrm{H}_{P, G}^{\lambda}: \mathrm{D}_{\zeta}\left({ }_{x, \infty} \widetilde{\operatorname{Bun}}_{\tilde{P}}\right) \rightarrow \mathrm{D}_{\zeta}\left({ }_{x, \infty} \widetilde{\operatorname{Bun}}_{\tilde{P}}\right)
$$

given by ${ }_{x} \mathrm{H}_{P, G}^{\lambda}(\mathcal{T})={ }_{x} \mathrm{H}_{P, G}^{\leftarrow}\left(\mathcal{A} \mathcal{\varepsilon}^{\lambda}, \mathcal{T}\right)$

For $\nu \in \Lambda_{M}^{\sharp,+}$ we define the perverse sheaf $\operatorname{IC}_{\nu, \zeta} \in \mathrm{D}_{\zeta}\left({ }_{x, \geq \nu} \widetilde{\operatorname{Bun}}_{\tilde{P}}\right)$ as follows. As in ([3], Section 4.2.3) we denote by

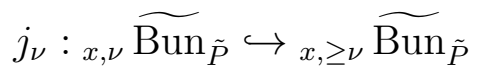

the corresponding open immersion. The stack ${ }_{x, \nu} \widetilde{\operatorname{Bun}}_{\tilde{P}}$ classifies $\left(\mathcal{F}_{M}^{1}, \mathcal{F}_{G}, \kappa\right) \in{ }_{x, 0} \widetilde{\operatorname{Bun}}_{P}$, $\mathcal{F}_{M} \in \operatorname{Bun}_{M}, \beta_{M}:\left.\mathcal{F}_{M} \widetilde{\widetilde{F}}_{M}^{1}\right|_{X-x}$ such that $\mathcal{F}_{M}$ is in the position $\nu$ with respect to $\mathcal{F}_{M}^{1}$ at $x,\left(\mathcal{F}_{M}, \mathcal{U}\right) \in \widetilde{\operatorname{Bun}}_{M},\left(\mathcal{F}_{G}, \mathcal{U}_{G}\right) \in \widetilde{\operatorname{Bun}}_{G}$. 
The projection $\widetilde{\operatorname{Bun}}_{\tilde{P}} \rightarrow{ }_{x, 0} \operatorname{Bun}_{P} \times_{\operatorname{Bun}_{G}} \widetilde{\operatorname{Bun}}_{G}$ sending the above point to $\left(\mathcal{F}_{M}^{1}, \mathcal{F}_{G}, \kappa, \mathcal{U}_{G}\right)$ is a locally trivial fibration (in smooth topology) with typical fibre $\widetilde{\mathrm{Gr}}_{M, x}^{\nu}$. As in Section 4.2, one gets the twisted exterior product

$$
\operatorname{IC}\left({ }_{x, 0} \operatorname{Bun}_{P} \times_{\operatorname{Bun}_{G}} \widetilde{\operatorname{Bun}}_{G}\right) \tilde{\otimes} \mathcal{A}_{M, \mathcal{E}}^{\nu}
$$

on $\widetilde{\operatorname{Bun}}_{\tilde{P}}$. Then $\mathrm{IC}_{\nu, \zeta}$ is defined as its intermediate extension under $j_{\nu}$. In particular, $\mathrm{IC}_{\zeta}=\mathrm{IC}_{0, \zeta}$ on ${ }_{x, \geq 0} \widetilde{\operatorname{Bun}}_{\tilde{P}}=\widetilde{\operatorname{Bun}}_{\tilde{P}}$.

The following are analogs of ([3], Theorem 4.1.3 and 4.1.5).

Proposition 4.4. For $\nu \in \Lambda_{M}^{\sharp,+}$ one has canonically ${ }_{x} \mathrm{H}_{P, M}^{\nu}\left(\mathrm{IC}_{\zeta}\right) \underset{\rightrightarrows}{\rightarrow} \mathrm{IC}_{-w_{0}^{M}(\nu), \zeta}$.

Proposition 4.5. For $\lambda \in \Lambda^{\sharp+}$ there is a canonical isomorphism

$$
{ }_{x} \mathrm{H}_{P, G}^{\lambda}\left(\mathrm{IC}_{\zeta}\right) \widetilde{\rightarrow} \underset{\nu \in \Lambda_{M}^{\sharp,+}}{\oplus} \mathrm{IC}_{\nu, \zeta} \otimes \operatorname{Hom}_{\check{M}_{n}}\left(U^{\nu}, V^{\lambda}\right) .
$$

Corollary 4.2. The two functors $\operatorname{Perv}_{G, n} \rightarrow \mathrm{D}_{\zeta}\left({ }_{x, \infty} \widetilde{\operatorname{Bun}}_{\tilde{P}}\right)$

$$
\mathcal{S} \mapsto{ }_{x} \mathrm{H}_{P, G}^{\leftarrow}\left(\mathcal{S}, \mathrm{IC}_{\zeta}\right) \quad \text { and } \quad \mathcal{S} \mapsto{ }_{x} \mathrm{H}_{P, M}\left(\mathrm{gRes}(\mathcal{S}), \mathrm{IC}_{\zeta}\right)
$$

are canonically isomorphic. This isomorphism is compatible with the tensor structure on $\mathbb{P e r v}_{G, n}$ as in ([3], Corollary 4.7).

Now as in ([3], Section 4.1.8) combination of Propositions 4.4 and 4.5 implies Theorem 2.4. The proof of Proposition 4.4 is completely analogous to ([3], Theorem 4.1.3).

4.4.2. Proof of Proposition 4.5. The proof of ([3], Theorem 4.1.5) applies in our situation with the role of ([3], Corollary 4.3.5) replaced by our Corollary 4.1.

For the convenience of the reader recall that in the proof of ([3], Theorem 4.1.5) for $\nu, \nu^{\prime}, \eta \in \Lambda_{M}^{+}, \lambda^{\prime} \in \Lambda_{G}^{+}$the following locally closed substack $Z_{P, G}^{\nu, \nu^{\prime}, \eta, \lambda^{\prime}} \hookrightarrow_{x, \infty} Z_{P, G}$ plays a key role. It classifies

$$
\left(\mathcal{F}_{M}^{1}, \mathcal{F}_{G}, \kappa\right) \in{ }_{x, 0} \widetilde{\operatorname{Bun}}_{P},\left.\mathcal{F}_{M} \widetilde{\rightarrow} \mathcal{F}_{M}^{1}\right|_{X-x},\left.\mathcal{F}_{M}^{2} \widetilde{\rightarrow} \mathcal{F}_{M}^{1}\right|_{X-x},\left.\mathcal{F}_{G}^{\prime} \widetilde{\rightarrow} \mathcal{F}_{G}\right|_{X-x}
$$

such that $\mathcal{F}_{M}$ is in the position $\nu$ w.r.t $\mathcal{F}_{M}^{1}$ at $x, \mathcal{F}_{M}^{2}$ is in the position $\eta$ w.r.t. $\mathcal{F}_{M}^{1}$ at $x, \mathcal{F}_{M}$ is in the position $\nu^{\prime}$ w.r.t. $\mathcal{F}_{M}^{2}$ at $x,\left(\mathcal{F}_{M}^{2}, \mathcal{F}_{G}^{\prime}, \kappa\right) \in{ }_{x, 0} \widetilde{\operatorname{Bun}}_{P}, \mathcal{F}_{G}^{\prime}$ is in the position $\lambda^{\prime}$ w.r.t. $\mathcal{F}_{G}$ at $x$.

It is included into the diagram

$$
\widetilde{\operatorname{Bun}}_{P} \stackrel{\prime h h_{G}^{\overleftarrow{G}}}{\leftarrow} Z_{P, G}^{\nu, \nu^{\prime}, \eta, \lambda^{\prime}} \stackrel{\prime h}{h}_{x, \nu^{\prime}} \widetilde{\operatorname{Bun}}_{P},
$$

where ' $h \overleftarrow{G}$ sends the above point to $\left(\left(\mathcal{F}_{M}^{1}, \mathcal{F}_{G}, \kappa\right) \in{ }_{x, 0} \widetilde{\operatorname{Bun}}_{P},\left.\mathcal{F}_{M} \widetilde{\rightarrow}_{\mathcal{F}_{M}}^{1}\right|_{X-x}\right)$. The map ${ }^{\prime} h_{G}$ sends the above point to

$$
\left(\left(\mathcal{F}_{M}^{2}, \mathcal{F}_{G}^{\prime}, \kappa\right) \in{ }_{x, 0} \widetilde{\operatorname{Bun}}_{P},\left.\mathcal{F}_{M} \widetilde{\rightrightarrows} \mathcal{F}_{M}^{2}\right|_{X-x}\right)
$$


In our situation we define $Z_{\tilde{P}, \tilde{G}}^{\nu, \nu^{\prime}, \eta, \lambda^{\prime}}$ by the base change ${ }_{x, \infty} Z_{\tilde{P}, \tilde{G}} \rightarrow_{x, \infty} Z_{P, G}$. Let $K^{\nu, \nu^{\prime}, \eta, \lambda^{\prime}}$ denote the !-direct image under

$$
{ }^{\prime} \tilde{h}_{G}^{\leftarrow}: Z_{\tilde{P}, \tilde{G}}^{\nu, \nu^{\prime}, \eta, \lambda^{\prime}} \rightarrow{ }_{x, \nu} \widetilde{\operatorname{Bun}}_{\tilde{P}}
$$

of the $*$-restriction of $\left(\mathcal{A}_{\varepsilon}^{-w_{0}(\lambda)} \tilde{\otimes} \mathrm{IC}_{\zeta}\right)^{r}$ to $Z_{\tilde{P}, \tilde{G}}^{\nu, \nu^{\prime}, \eta, \lambda^{\prime}}$. As in $([3]$, Section 4.3.8) one shows the following.

a) The complex $K^{\nu, \nu^{\prime}, \eta, \lambda^{\prime}}$ is placed in perverse degrees $\leq 0$, and the inequality is strict unless $\nu^{\prime}=0, \lambda^{\prime}=\lambda$ and $\nu=\eta$.

b) The $*$-restriction of $K^{\nu, 0, \nu, \lambda}$ to ${ }_{x, \nu} \widetilde{\operatorname{Bun}}_{\tilde{P}}-{ }_{x, \nu} \operatorname{Bun}_{\tilde{P}}$ is placed in structly negative perverse degrees.

c) The 0-th perverse cohomology sheaf of $K^{\nu, 0, \nu, \lambda}$ over ${ }_{x, \nu} \operatorname{Bun}_{\tilde{P}}$ identifies canonically with $\mathrm{IC}_{\nu, \zeta} \otimes \operatorname{Hom}_{\check{M}_{n}}\left(U^{\nu}, V^{\lambda}\right)$.

Point c) here uses Corollary 4.1, We are done.

4.5. Description of $\mathrm{IC}_{\zeta}$. In this section we give a description of $\mathrm{IC}_{\zeta}$ generalizing the main result of [2] to our twisted setting.

We freely use some notations of [2]. In particular, $\Lambda_{G, P}^{\text {pos }}$ is the $\mathbb{Z}_{+}$-span of $\left\{\alpha_{i}, i \in \mathcal{J}-\mathcal{J}_{M}\right\}$ in $\Lambda_{G, P}$. If $\theta \in \Lambda_{G, P}^{\text {pos }}$ is the projection under $\Lambda \rightarrow \Lambda_{G, P}$ of $\tilde{\theta} \in \operatorname{Span}\left(\alpha_{j}\right), j \in \mathcal{J}-\mathcal{J}_{M}$ then $b(\theta)=w_{0}^{M}(\tilde{\theta})$. Here $w_{0}^{M}$ is the longest element of the Weyl group of $M$. For $\theta \in \Lambda_{G, P}^{\text {pos }}$ the scheme $\mathrm{Gr}_{M}^{+, \theta}$ is defined in ([2], Proposition 1.7).

Let $\check{\mathfrak{u}}_{n}$ denote the Lie algebra of the unipotent radical of the Borel subgroup $\check{B}_{n} \subset \check{G}_{n}$. More generally, let $\check{\mathfrak{u}}_{n}(P)$ denote the Lie algebra of the unipotent radical of the standard parabolic $\check{P}_{n} \subset \check{G}_{n}$ corresponding to $\mathcal{J}_{M} \subset \mathcal{J}$.

For $\nu \in C^{*}\left(\check{M}_{n}\right)$ and $V \in \operatorname{Rep}\left(\check{M}_{n}\right)$ write $V_{\nu}$ for the direct summand of $V$, on which $Z\left(\check{M}_{n}\right)$ acts by $\nu$. In particular, we have the $\check{M}_{n}$-module $\left(\check{\mathfrak{u}}_{n}(P)\right)_{\nu}$.

Lemma 4.1. If $\nu \in C^{*}\left(\check{M}_{n}\right)$ and $\left(\check{\mathfrak{u}}_{n}(P)\right)_{\nu}$ is not zero then it is an irreducible $\check{M}_{n}$-module.

Proof Each root space of $\check{T}_{n}$ in $\check{\mathfrak{u}}_{n}(P)$ is 1-dimensional, so our claim follows from Lemma 4.2 below.

Lemma 4.2. Let $H$ be a connected reductive group over $k, \check{H}$ be the Langlands dual over $\overline{\mathbb{Q}}_{\ell}$. Let $\nu_{1}, \nu_{2}$ be dominant coweights of $H$ such that $\nu_{1}=\nu_{2}$ in $\pi_{1}(H)$. Then the irreducible $\check{H}$-representations $V^{\nu_{1}}, V^{\nu_{2}}$ with highest weights $\nu_{1}, \nu_{2}$ admit a common weight.

Proof Let $\theta$ be the image of $\nu_{i}$ in $\pi_{1}(H)$. If $\theta=0$ then they both admit the zero weight space. Assume $\theta \neq 0$. Let $\mu_{i}$ be a dominant coweight of $H$ satisfying $\mu_{i} \leq \nu_{i}$ and minimal with this property. Then the orbit $\mathrm{Gr}_{H}^{\mu_{i}}$ in closed in the connected component $\mathrm{Gr}_{H}^{\theta}$ of the affine grassmanian of $H$. Since $\operatorname{Gr}_{H}^{\theta}$ admits a unique closed $H(\mathbf{O})$-orbit, $\mu_{1}=\mu_{2}$.

Recall the functor Loc $: \operatorname{Rep}\left(\check{M}_{n}\right) \widetilde{\rightarrow} \operatorname{Perv}_{M, G, n}^{\natural}$ from Section 4.2.

Lemma 4.3. Let $\nu \in \Lambda_{M}^{\sharp,+}$ be such that the irreducible $\check{M}_{n}$-module $U^{\nu}$ appears in $\check{\mathfrak{u}}_{n}(P)$, let $\theta$ be the image of $\nu$ in $\Lambda_{G, P}^{\text {pos }}$. Then $\operatorname{Loc}\left(U^{\nu}\right)$ over $\widetilde{\mathrm{Gr}}_{M}$ is the extension by zero from $\widetilde{\mathrm{Gr}}_{M}^{+, \theta}$. 
Proof Note that $\nu$ lies in $\mathbb{Z}_{+}$-span of positive coroots of $G$. Let $\tilde{\theta}$ be the unique element in $\mathbb{Z}_{+}$-span of $\left\{\alpha_{i} \mid i \in \mathcal{J}-\mathcal{J}_{M}\right\}$ such that $\tilde{\theta}=\nu$ in $\Lambda_{G, P}$. So, $\nu=\tilde{\theta}+\nu_{1}$, where $\nu_{1}$ is in $\mathbb{Z}_{+}$-span of positive coroots of $M$. Now $w_{0}^{M}(\nu)$ is a positive root of $\check{G}_{n}$, and $w_{0}^{M}(\nu)=\nu$ in $\Lambda_{G, P}$. So, $\tilde{\theta} \leq_{M} w_{0}^{M}(\nu)$. This implies $\nu \leq_{M} w_{0}^{M}(\tilde{\theta})=b(\theta)$.

Set

$$
J=\left\{\nu \in C^{*}\left(\check{M}_{n}\right) \mid\left(\check{\mathfrak{u}}_{n}(P)\right)_{\nu} \neq 0\right\}
$$

Lemma 4.4. The restriction of $c_{P}: C^{*}\left(\check{M}_{n}\right) \rightarrow \Lambda_{G, P}^{\sharp}$ to $J$ is injective.

Proof Let $C_{r}^{*}\left(\check{M}_{n}\right) \subset C^{*}\left(\check{M}_{n}\right)$ be the subgroup generated by roots of $\check{G}_{n}$. It is a free abelian group, so the intersection of $C_{r}^{*}\left(\check{M}_{n}\right)$ with the kernel of $c_{P}$ is $\{0\}$. The restriction of $c_{P}$ to $C_{r}^{*}\left(\check{M}_{n}\right)$ is injective, and one has $J \subset C_{r}^{*}\left(\check{M}_{n}\right)$.

Set $\Lambda_{G, P}^{\sharp, p o s}=\Lambda_{G, P}^{\sharp} \cap \Lambda_{G, P}^{\text {pos }}$. For $\theta \in \Lambda_{G, P}^{\sharp}$ and $V \in \operatorname{Rep}\left(\check{M}_{n}\right)$ set

$$
V_{\theta}=\underset{\nu \in C^{*}\left(\check{M}_{n}\right), c_{P}(\nu)=\theta}{\oplus} V_{\nu}
$$

Remark 4.1. For $i \in \mathcal{J}$ let $\delta_{i}$ denote the denominator of $\frac{\iota\left(\alpha_{i}, \alpha_{i}\right)}{2 n}$. Recall that $\delta_{i} \alpha_{i}$ is the corresponding simple root of $\check{G}_{n}$. If $i \in \mathcal{J}-\mathcal{J}_{M}$ then $\delta_{i} \alpha_{i} \in J$. The set $J$ may contain other elements also.

4.5.1. Given $\theta \in \Lambda_{G, P}^{\text {pos }}$, let $\mathfrak{U}(\theta)$ be a decomposition of $\theta$ as in ([2], Section 1.4), recall the isomorphism of ([2], Proposition 1.9)

$$
\mathfrak{U}(\theta) \widetilde{\operatorname{Bun}}_{P} \widetilde{\rightarrow} \operatorname{Bun}_{P} \times_{\operatorname{Bun}_{M}} \mathcal{H}_{M}^{+, \mathfrak{U}(\theta)}
$$

Let $\mathfrak{u}(\theta) \widetilde{\operatorname{Bun}}_{\tilde{P}}$ be obtained from $\mathfrak{u}(\theta) \widetilde{\operatorname{Bun}}_{P}$ by the base change $\widetilde{\operatorname{Bun}}_{\tilde{P}} \rightarrow \widetilde{\operatorname{Bun}}_{P}$. We will describe the $*$-restriction of $\mathrm{IC}_{\zeta}$ under $\mathfrak{U}(\theta) \widetilde{\operatorname{Bun}}_{\tilde{P}} \hookrightarrow \widetilde{\operatorname{Bun}}_{\tilde{P}}$.

Recall that if $\mathfrak{U}(\theta)$ is the decomposition

$$
\theta=\sum_{m} n_{m} \theta_{m}
$$

then $|\mathfrak{U}(\theta)|=\sum_{m} n_{m}, X^{\mathfrak{U}(\theta)}=\prod_{m} X^{\left(n_{m}\right)}$, and $\stackrel{\circ}{X^{\mathfrak{U}}(\theta)}$ is the complement to all the diagonals

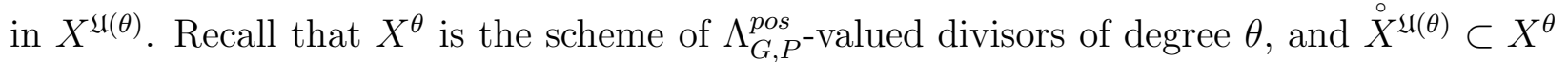
is locally closed. Set ${ }^{u n s} \stackrel{\circ}{X}^{\mathfrak{U}(\theta)}=X^{\mathfrak{U}(\theta)}-\Delta$, where $\Delta$ is the divisor of all the diagonals. Here uns stands for 'unsymmetrized'.

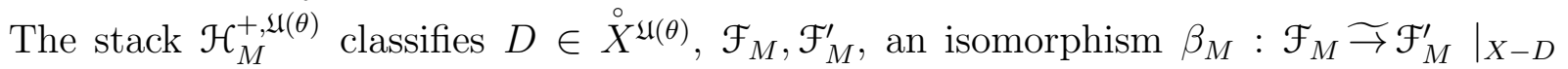
such that for each $\mathcal{V} \in \operatorname{Rep}(G)$ the induced map

$$
\beta_{M}^{\mathcal{V}}: \mathcal{V}_{\mathcal{F}_{M}}^{U(P)} \hookrightarrow \mathcal{V}_{\mathcal{F}_{M}^{\prime}}^{U(P)}
$$

is an inclusion, and $\beta_{M}$ induces an isomorphism

$$
\mathcal{F}_{M /[M, M]} \widetilde{\rightarrow} \mathcal{F}_{M /[M, M]}^{\prime}(-D)
$$


Note that the Plucker relations for $\beta_{M}^{\mathcal{D}}$ hold automatically. So, here we think of $\mathcal{F}_{M}^{\prime}$ as the 'background' $M$-torsor. The stack (22) classifies the same data together with a $P$-torsor $\mathcal{F}_{P}^{\prime}$ and an isomorphism $\mathcal{F}_{P}^{\prime} \times_{P} M \widetilde{\rightarrow} \mathcal{F}_{M}^{\prime}$.

Let $\mathcal{H}_{\tilde{M}}^{+, \mathfrak{U}(\theta)}$ be obtained from $\mathcal{H}_{M}^{+, \mathfrak{U}(\theta)}$ by the base change $\widetilde{\operatorname{Bun}}_{M} \times \widetilde{\operatorname{Bun}}_{M} \rightarrow \operatorname{Bun}_{M} \times \operatorname{Bun}_{M}$. So, it classifies the same data together with lines $\mathcal{U}, \mathcal{U}^{\prime}$ equipped with

$$
\mathcal{U}^{N} \widetilde{\rightrightarrows}\left(\mathcal{L}_{M}\right)_{\mathcal{F}_{M}}, \quad \mathcal{U}^{\prime N} \underset{\rightrightarrows}{\rightarrow}\left(\mathcal{L}_{M}\right)_{\mathcal{F}_{M}^{\prime}}
$$

We get

$$
\mathfrak{u}(\theta) \widetilde{\operatorname{Bun}}_{\tilde{P}} \widetilde{\rightarrow} \operatorname{Bun}_{P} \times \operatorname{Bun}_{M} \mathcal{H}_{\tilde{M}}^{+, \mathfrak{U}(\theta)}
$$

Note that $\left(a, a^{\prime}\right) \in \mu_{N}(k) \times \mu_{N}(k) \subset \operatorname{Aut}(\mathcal{U}) \times \operatorname{Aut}\left(\mathcal{U}^{\prime}\right)$ acts on $\left.\operatorname{IC}_{\zeta}\right|_{\mathfrak{U}(\theta)} \widetilde{\operatorname{Bun}_{\tilde{P}}}$ as $\zeta\left(\frac{a^{\prime}}{a}\right)$.

Let $\mathrm{Gr}_{M}^{+, \mathfrak{U}(\theta)}$ be as in [2], so it is obtained from $\mathcal{H}_{M}^{+, \mathfrak{U}(\theta)}$ by the base change $\operatorname{Spec} k \rightarrow \operatorname{Bun}_{M}$ trivializing the $M$-torsor $\mathcal{F}_{M}^{\prime}$. We also denote by $\mathcal{L}_{X}$ the line bundle on $\mathrm{Gr}_{M}^{+, \mathfrak{U}(\theta)}$ whose fibre at $\left(\mathcal{F}_{M}, \beta_{M}, D\right)$ is

$$
\operatorname{det} \operatorname{R} \Gamma(X, \mathfrak{g} \otimes \mathcal{O}) \otimes \operatorname{det} \mathrm{R} \Gamma\left(X, \mathfrak{g}_{\mathcal{F}_{M}}\right)^{-1}
$$

Let $\widetilde{\mathrm{Gr}}_{M}^{+, \mathfrak{U}(\theta)}$ be the gerb of $N$-th roots of $\mathcal{L}_{X}$ over $\mathrm{Gr}_{M}^{+, \mathfrak{U}(\theta)}$.

As in ([3], Section 6.2.3) we set $\Lambda_{M, G}^{+}=\Lambda_{M}^{+} \cap w_{0}^{M}\left(\Lambda_{G}^{\text {pos }}\right)$. Say that $V \in \operatorname{Rep}\left(\check{M}_{n}\right)$ is positive if $\operatorname{Loc}(V)$ is the extension by zero from $\widetilde{\mathrm{Gr}}_{M}^{+}=\cup_{\theta \in \Lambda_{G, P}^{p o s}} \widetilde{\mathrm{Gr}}_{M}^{+, \theta}$. In this case it is actually the extension by zero from $\cup_{\theta \in \Lambda_{G, P}^{\sharp, p o s}}{\widetilde{\mathrm{Gr}_{M}}}_{M}^{+, \theta}$.

In fact, $V \in \operatorname{Rep}\left(\check{M}_{n}\right)$ is positive if and only if for any irreducible subrepresentation $U^{\nu} \subset V, \nu \in \Lambda_{M}^{\sharp,+}$ there is $\mu \in \Lambda_{M, G}^{+}$such that $\nu \leq_{M} \mu$.

Let ${ }^{\text {uns }} \widetilde{\mathrm{Gr}}_{M}^{+, \mathfrak{U}(\theta)}$ be obtained from $\widetilde{\mathrm{Gr}}_{M}^{+, \mathfrak{U}(\theta)}$ by the base change ${ }^{\text {uns }} \stackrel{\circ}{X}^{\mathfrak{U}(\theta)} \rightarrow \stackrel{\circ}{X}^{\mathfrak{U}(\theta)}$.

For $V \in \operatorname{Rep}\left(\check{M}_{n}\right)$ positive we denote by $\operatorname{Loc}_{\zeta}^{\mathfrak{U}(\theta)}(V)$ the perverse sheaf on $\widetilde{\mathrm{Gr}}_{M}^{+, \mathfrak{U}(\theta)}$, on which $\mu_{N}(k)$ acts by $\zeta$ and such that for $D=\sum \theta_{k} x_{k} \in \stackrel{\circ}{X^{u}(\theta)}$ its restriction to

$$
\prod_{k} \widetilde{\mathrm{Gr}}_{M, x_{k}}^{+, \theta_{k}}
$$

is

$$
\left(\bigotimes_{k} \operatorname{Loc}_{\zeta}\left(V_{\theta_{k}}\right)\right) \otimes \overline{\mathbb{Q}}_{\ell}[|\mathfrak{U}(\theta)|]
$$

To make this definition rigorous, we first define $\operatorname{Loc}_{\zeta}^{u n s, \mathfrak{U}(\theta)}(V)$ on ${ }^{\text {uns }} \widetilde{\mathrm{Gr}}_{M}^{+, \mathfrak{U}(\theta)}$. Write ${ }^{\text {uns }} \underset{\mathrm{Gr}_{M}^{+, \mathfrak{U}(\theta)}}{\widetilde{\sigma^{2}}}$ for the stack over ${ }^{u n s} \stackrel{\circ}{X}^{\mathfrak{U}(\theta)}$ whose fibre over $D \in{ }^{u n s} \stackrel{\circ}{X}^{\mathfrak{U}(\theta)}$ is (25). The desired perverse sheaf is obtained by descent via the gerb

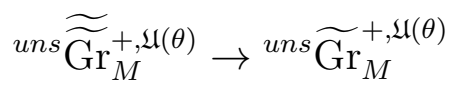

Over uns $\widetilde{\widetilde{G r}}_{M}^{+, \mathfrak{U}(\theta)}$ this perverse sheaf is defined similarly using the fact (cf. [9], Section 4.1.2) that every object of $\operatorname{Perv}_{M, G, n}$ admits a unique $\operatorname{Aut}_{2}^{0}(\mathbf{O})$-equivariant structure. Here $\operatorname{Aut}_{2}^{0}(\mathbf{O})$ 
is a connected group scheme defined in ([9], Section 2.3). More precisely, consider the torsor over ${ }^{\text {uns }} \stackrel{\circ}{X}^{\mathfrak{U}(\theta)}$ whose fibre over $D$ is the set of isomorphisms $\left(\mathbf{O}_{x}, \mathcal{E}_{X}\left(\mathbf{O}_{x}\right)\right) \widetilde{\rightrightarrows}(\mathcal{O}, \mathcal{E})$ for all $x \in D$. Here $\mathcal{E} \in \Omega(\mathbf{O})^{\frac{1}{2}}$ is the object we picked in Section 3.2. Then ${ }^{\text {uns }} \widetilde{\widetilde{G r}}_{M}^{+, \mathfrak{U}(\theta)}$ is the twist of $\prod_{k} \widetilde{G r}_{M}^{+, \theta_{k}}$ by this torsor. So, the object $\operatorname{Loc}_{\zeta}^{u n s, \mathfrak{U}(\theta)}(V)$ is well-defined, and moreover equivariant with respect to the Galois group of the covering ${ }^{\text {uns }} \stackrel{\circ}{X}^{\mathfrak{U}(\theta)} \rightarrow \stackrel{\circ}{X}^{\mathfrak{U}(\theta)}$. Thus, it gives rise to the perverse sheaf $\operatorname{Loc}_{\zeta}^{\mathfrak{U}(\theta)}(V)$ on $\widetilde{\mathrm{Gr}}_{M}^{+, \mathfrak{U}(\theta)}$ defined up to a unique isomorphism.

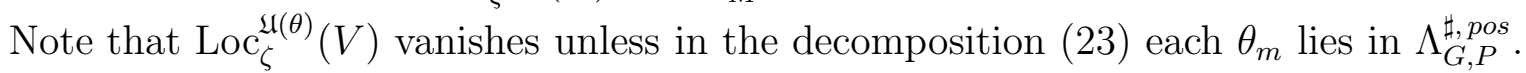

4.5.2. Let $\operatorname{Bun}_{M, \mathfrak{U}(\theta)}$ be the stack classifying $\mathcal{F}_{M} \in \operatorname{Bun}_{M}, D \in \stackrel{\circ}{X}^{\mathfrak{u}(\theta)}$, and a trivialization of $\mathcal{F}_{M}$ over the formal neighbourhood of $D$. Let $\widetilde{\operatorname{Bun}}_{M, \mathfrak{U}(\theta)}=\operatorname{Bun}_{M, \mathfrak{U}(\theta)} \times_{\operatorname{Bun}_{M}} \widetilde{\operatorname{Bun}}_{M}$.

Let $M_{\mathfrak{U}(\theta)}$ be the scheme classifying $D \in \stackrel{\circ}{X}^{\mathfrak{u}(\theta)}$ and a section of $M$ over the formal neighbourhood of $D$. This is a group scheme over $\stackrel{\circ}{X}^{u(\theta)}$. The group $M_{\mathfrak{U}(\theta)}$ acts diagonally on $\operatorname{Bun}_{M, \mathfrak{U}(\theta)} \times_{\stackrel{\circ}{X}^{u(\theta)}} \mathrm{Gr}_{M}^{+, \mathfrak{U}(\theta)}$, and the stack quotient is denoted

$$
\operatorname{Bun}_{M, \mathfrak{U}(\theta)} \times_{M_{\mathfrak{U}(\theta)}} \operatorname{Gr}_{M}^{+, \mathfrak{U}(\theta)}
$$

There is an isomorphism $\gamma^{\rightarrow}$ from the latter stack to $\mathcal{H}_{M}^{+, \mathfrak{U}(\theta)}$ such that the projection to the first term corresponds to $h_{M}$. As above, one extends this $M_{\mathfrak{U}(\theta)}$-torsor to a $M_{\mathfrak{U}(\theta)}$-torsor

$$
\tilde{\gamma} \rightarrow \widetilde{\operatorname{Bun}}_{M, \mathfrak{U}(\theta)} \times_{\stackrel{o}{X}^{u(\theta)}}{\widetilde{\mathrm{Gr}_{M}}}^{+, \mathfrak{U}(\theta)} \rightarrow \mathcal{H}_{\tilde{M}}^{+, \mathfrak{U}(\theta)}
$$

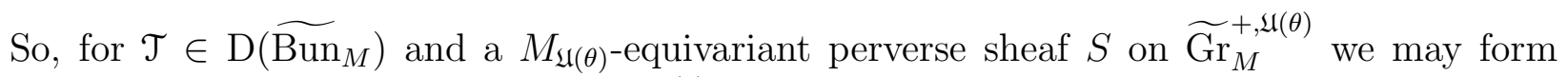
their twisted product $(\mathcal{T} \tilde{\otimes} S)^{r}$ on $\mathcal{H}_{\tilde{M}}^{+, \mathfrak{U}(\theta)}$. For $V \in \operatorname{Rep}\left(\check{M}_{n}\right)$ positive define

$$
\operatorname{Loc}_{\operatorname{Bun}_{M}, \zeta}^{\mathfrak{U}(\theta)}(V)=\left(\operatorname{IC}\left(\widetilde{\operatorname{Bun}_{M}}\right) \tilde{\otimes} \operatorname{Loc}_{\zeta}^{\mathfrak{U}(\theta)}(V)\right)^{r}
$$

Similarly, applying for $\nu_{P}: \operatorname{Bun}_{P} \rightarrow \operatorname{Bun}_{M}$ the functor $\nu_{P}^{*}\left[\operatorname{dim} \operatorname{rel}\left(\nu_{P}\right)\right]$ to (26) , we get the perverse sheaf on (24) denoted

$$
\operatorname{Loc}_{B \operatorname{Bun}_{P}, \zeta}^{\mathfrak{U}(\theta)}(V)
$$

Theorem 4.1. The *-restriction of $\mathrm{IC}_{\zeta}$ under $\mathfrak{u}(\theta) \widetilde{\operatorname{Bun}}_{\tilde{P}} \hookrightarrow \widetilde{\operatorname{Bun}}_{\tilde{P}}$ vanishes unless in the decomposition (23) each $\theta_{m}$ lies in $\Lambda_{G, P}^{\sharp, p o s}$. In the latter case it is isomorphic to

$$
\operatorname{Loc}_{\operatorname{Bun}_{P}, \zeta^{-1}}^{\mathfrak{U}(\theta)}\left(\underset{i \geq 0}{\oplus} \operatorname{Sym}^{i}\left(\check{\mathfrak{u}}_{n}(P)\right)[2 i]\right) \otimes \overline{\mathbb{Q}}_{\ell}[-|\mathfrak{U}(\theta)|]
$$

where $\underset{i \geq 0}{\oplus} \operatorname{Sym}^{i}\left(\check{\mathfrak{u}}_{n}(P)\right)[2 i]$ is viewed as a cohomologically graded $\check{M}_{n}$-module. 


\section{Proof of Theorem 4.1}

5.1. Zastava spaces. Keep notations of Section 4. We also use some notations from [2]. For $\theta \in \Lambda_{G, P}^{\text {pos }}$ let $Z^{\theta}$ be as in [2]. Recall that $\operatorname{Mod}_{M}^{+, \theta}$ classifies $\left(D \in X^{\theta}, \mathcal{F}_{M} \in \operatorname{Bun}_{M}, \beta_{M}\right)$, where $\beta_{M}:\left.\mathcal{F}_{M} \widetilde{\rightarrow} \mathcal{F}_{M}^{0}\right|_{X-D}$ is an isomorphism such that for any $G$-module $\mathcal{V}$, the map

$$
\beta_{M}: \mathcal{V}_{\mathcal{F}_{M}}^{U(P)} \rightarrow \mathcal{V}_{\mathcal{F}_{M}^{0}}^{U(P)}
$$

is regular over $X$, and $\beta_{M}$ induces $\mathcal{F}_{M /[M, M]} \underset{\rightarrow}{\rightarrow} \mathcal{F}_{M /[M, M]}^{0}(-D)$.

A point of $Z^{\theta}$ is given by

$$
\left(\mathcal{F}_{G}, \mathcal{F}_{M}, \beta, \beta_{M}, D\right),
$$

where $\left(\mathcal{F}_{M}, \beta_{M}, D\right) \in \operatorname{Mod}_{M}^{+, \theta}$, and $\mathcal{F}_{G}$ is a $G$-torsor on $X$ equipped with a trivialization $\beta:\left.\mathcal{F}_{G} \widetilde{\rightarrow} \mathcal{F}_{G}^{0}\right|_{X-D}$ satisfying the conditions of ([2], Section 2.2). We have a diagram $Z^{\theta} \stackrel{\pi_{P}}{\rightarrow}$ $\operatorname{Mod}_{M}^{+, \theta} \rightarrow \operatorname{Bun}_{M}$, where the second map sends the above point to $\mathcal{F}_{M}$. By abuse of notations, the restrictions of $\mathcal{L}_{M}$ under these maps are also denoted by $\mathcal{L}_{M}$. Let

$$
\widetilde{Z}^{\theta} \stackrel{\pi_{P}}{\rightarrow} \operatorname{Mod}_{\tilde{M}}^{+, \theta} \rightarrow{\widetilde{\operatorname{Bun}_{M}}}
$$

be obtained from the latter diagram by the base change $\widetilde{\operatorname{Bun}}_{M} \rightarrow \operatorname{Bun}_{M}$. A point of $\widetilde{Z}^{\theta}$ is given by (27) together with a line $\mathcal{U}$ equipped with $\mathcal{U}^{N} \widetilde{\Im}\left(\mathcal{L}_{M}\right)_{\mathcal{F}_{M}}$.

The open subscheme $Z_{\max }^{\theta} \subset Z^{\theta}$ is defined in ([2], Section 2.2). Let $\widetilde{Z}_{\max }^{\theta}=Z_{\max }^{\theta} \times_{Z^{\theta}} \widetilde{Z}^{\theta}$. We have an isomorphism

$$
i_{\text {max }}: B\left(\mu_{N}\right) \times Z_{\text {max }}^{\theta} \widetilde{\rightarrow} \widetilde{Z}_{\text {max }}^{\theta}
$$

sending $\mathcal{U}_{0}, \mathcal{U}_{0}^{N} \widetilde{\rightarrow} k$ and $\left(\mathcal{F}_{G}, \mathcal{F}_{M}, \beta, \beta_{M}\right)$ to $\left(\mathcal{F}_{G}, \mathcal{F}_{M}, \beta, \beta_{M}, \mathcal{U}\right)$, where $\mathcal{U}=\mathcal{U}_{0}^{-1}$ is equipped with the induced isomorphism

$$
\mathcal{U}^{N} \stackrel{\widetilde{\rightarrow}}{\stackrel{\sim}{\rightarrow}} \mathcal{L}_{\mathcal{F}_{G}} \widetilde{\rightarrow}\left(\mathcal{L}_{M}\right)_{\mathcal{F}_{M}}
$$

Define $\mathrm{IC}_{Z^{\theta}, \zeta}$ as the intermediate extension of $i_{\max *}\left(\mathcal{L}_{\zeta} \otimes \operatorname{IC}\left(Z_{\max }^{\theta}\right)\right)$ to $\tilde{Z}^{\theta}$. We underline that $a \in \mu_{N}(k) \subset \operatorname{Aut}(\mathcal{U})$ acts on $\mathrm{IC}_{Z^{\theta}, \zeta}$ as $\zeta^{-1}(a)$.

5.1.1. Action of $M_{X^{\theta}}$. For $D \in X^{\theta}$ denote by $\bar{D}$ (resp., by $\bar{D}^{0}$ ) the formal (resp., the punctured formal) neighbourhood of $D$ in $X$. This means that we pick a homomorphism of semigroups $\Lambda_{G, P}^{\text {pos }} \rightarrow \mathbb{Z}_{+}$sending each $\alpha_{i}, i \in \mathcal{J}-\mathcal{J}_{M}$ to a nonzero element, it yields a morphism $v: X^{\theta} \rightarrow X^{(d)}$, where $d$ is the image of $\theta$, and $\bar{D}$ is the formal neighbourhood of $v(D)$ in $X$. Similarly for $\bar{D}^{0}$.

Let $M_{X^{\theta}}$ be the scheme classifying $D \in X^{\theta}$ and a section of $M$ over the formal neighbourhood of $D$ in $X$.

The space $\operatorname{Mod}_{M}^{+, \theta}$ can be rewritten as the space classifying $D \in X^{\theta}$, a $M$-torsor $\mathcal{F}_{M}$ on $\bar{D}$, its trivialization $\beta_{M}:\left.\mathcal{F}_{M} \widetilde{\rightarrow} \mathcal{F}_{M}^{0}\right|_{\bar{D}^{0}}$ such that for each representation $\mathcal{V}$ of $M$ the map $\beta_{M}$ : $\mathcal{V}_{\mathcal{F}_{M}}^{U(P)} \rightarrow \mathcal{V}_{\mathcal{F}_{M}^{0}}^{U(P)}$ is regular over $\bar{D}$, and $\beta_{M}$ induces an isomorphism $\mathcal{F}_{M /[M, M]} \underset{\rightarrow}{\rightarrow} \mathcal{F}_{M /[M, M]}^{0}(-D)$ over $\bar{D}$. In this incarnation $M_{X^{\theta}}$ acts on $\operatorname{Mod}_{M}^{+, \theta}$ over $X^{\theta}$ by changing the trivialization $\beta_{M}$. 
Similarly, $Z^{\theta}$ can be seen as the scheme classifying $D \in X^{\theta}$, a $M$-torsor $\mathcal{F}_{M}$ on $\bar{D}$, its trivialization $\beta_{M}:\left.\mathcal{F}_{M} \widetilde{\rightarrow} \mathcal{F}_{M}^{0}\right|_{\bar{D}^{0}}$ such that for each representation $\mathcal{V}$ of $M$ the map $\beta_{M}$ : $\mathcal{V}_{\mathcal{F}_{M}}^{U(P)} \rightarrow \mathcal{V}_{\mathcal{F}_{M}^{0}}^{U(P)}$ is regular over $\bar{D}$, and $\beta_{M}$ induces an isomorphism $\mathcal{F}_{M /[M, M]} \widetilde{\rightarrow} \mathcal{F}_{M /[M, M]}^{0}(-D)$ over $\bar{D}$; a $G$-torsor $\mathcal{F}_{G}$ over $\bar{D}$, an isomorphism $\beta:\left.\mathcal{F}_{G} \widetilde{\rightarrow} \mathcal{F}_{G}^{0}\right|_{\bar{D}^{0}}$ such that for each $G$-module $V$ the map

$$
V_{\mathcal{F}_{G}} \stackrel{\beta}{\rightarrow} V_{\mathcal{F}_{G}^{0}} \rightarrow\left(V_{U\left(P^{-}\right)}\right)_{\mathcal{F}_{M}^{0}}
$$

is regular and surjective over $\bar{D}$, and the map

$$
V_{\mathcal{F}_{M}}^{U(P)} \stackrel{\beta_{M}}{\rightarrow} V_{\mathcal{F}_{M}^{0}}^{U(P)} \hookrightarrow V_{\mathcal{F}_{G}^{0}} \stackrel{\beta^{-1}}{\rightarrow} V_{\mathcal{F}_{G}}
$$

is regular over $\bar{D}$. In this incarnation $M_{X^{\theta}}$ acts on $Z^{\theta}$ via its action on $\mathcal{F}_{M}^{0}$. Namely, if $g$ is automorphism of $\mathcal{F}_{M}^{0}$ over $\bar{D}$, it sends the above point to the collection $\left(\mathcal{F}_{G}, \mathcal{F}_{M}, g \beta_{M}, g \beta\right)$.

The line bundle $\mathcal{L}_{M}$ on $Z^{\theta}$ is naturally $M_{X^{\theta}}$-equivariant. Namely, the fibre of $\mathcal{L}_{M}$ at $\left(\mathcal{F}_{G}, \mathcal{F}_{M}, \beta_{M}, \beta\right)$ is $\operatorname{det} \mathrm{R} \Gamma\left(X, \mathfrak{g}_{\mathcal{F}_{M}^{0}}\right) \otimes \operatorname{det} \mathrm{R} \Gamma\left(X, \mathfrak{g}_{\mathcal{F}_{M}}\right)^{-1}$, and $M_{X^{\theta}}$ acts via its action on $\mathcal{F}_{M}^{0}$. So, $M_{X^{\theta}}$ acts on $\operatorname{Mod}_{\tilde{M}}^{+, \theta}$ and on $\widetilde{Z}^{\theta}$, and the $\operatorname{maps}_{\mathfrak{s}}^{\theta}: \operatorname{Mod}_{\tilde{M}}^{+, \theta} \rightarrow \widetilde{Z}^{\theta}$ and $\pi_{P}: \widetilde{Z}^{\theta} \rightarrow \operatorname{Mod}_{\tilde{M}}^{+, \theta}$ are $M_{X^{\theta}}$-equivariant. Note that $\mathrm{IC}_{Z^{\theta}, \zeta}$ is naturally $M_{X^{\theta}}$-equivariant.

5.1.2. The relation between $\mathrm{IC}_{Z^{\theta}, \zeta}$ and $\mathrm{IC}_{\zeta}$. Write $\operatorname{Bun}_{M, X^{\theta}}$ for the stack classifying $\mathcal{F}_{M} \in$ $\operatorname{Bun}_{M}, D \in X^{\theta}$ and a trivialization of $\mathcal{F}_{M}$ over the formal neighbourhood of $D$ in $X$. Let $\widetilde{\operatorname{Bun}}_{M, X^{\theta}}$ be obtained from it by the base change $\widetilde{\operatorname{Bun}}_{M} \rightarrow \operatorname{Bun}_{M}$.

Recall that $Z_{\mathrm{Bun}_{M}}^{\theta}$ is defined as $Z^{\theta}$ by replacing $\mathcal{F}_{M}^{0}$ by the 'background' $M$-torsor $\mathcal{F}_{M}^{\prime} \in$ $\operatorname{Bun}_{M}$. Let $\widetilde{Z}_{\widetilde{\operatorname{Bun}}_{M}}^{\theta}$ be obtained from $Z_{\mathrm{Bun}_{M}}^{\theta}$ by adding lines $\mathcal{U}, \mathcal{U}^{\prime}$ equipped with isomorphisms $\mathcal{U}^{N} \widetilde{\Im}\left(\mathcal{L}_{M}\right)_{\mathcal{F}_{M}}, \mathcal{U}^{\prime n} \widetilde{\Im}\left(\mathcal{L}_{M}\right)_{\mathcal{F}_{M}^{\prime}}$, where $\mathcal{F}_{M}^{\prime}$ is the background $M$-torsor.

Let $M_{X^{\theta}}$ act diagonally on $\widetilde{Z}^{\theta} \times_{X^{\theta}} \widetilde{\operatorname{Bun}}_{M, X^{\theta}}$. As n Section 3.2 , we have a $M_{X^{\theta}}$-torsor

$$
\gamma_{Z}: \widetilde{Z}^{\theta} \times_{X^{\theta}} \widetilde{\operatorname{Bun}}_{M, X^{\theta}} \rightarrow \widetilde{Z}_{\widetilde{\operatorname{Bun}}_{M}}^{\theta}
$$

We form the twisted external product

$$
\left(\mathrm{IC}_{Z^{\theta}, \zeta} \tilde{\otimes} \mathrm{IC}\left(\widetilde{\operatorname{Bun}}_{M}\right)\right)
$$

on $\widetilde{Z}_{\widetilde{\operatorname{Bun}}_{M}}^{\theta}$, which is the descent with respect to $\gamma_{Z}$.

Let $\operatorname{Bun}_{P^{-}}^{r}$ be defined as in ([2], Section 3.6) so that $\operatorname{Bun}_{P^{-}}^{r} \rightarrow \operatorname{Bun}_{G}$ is smooth. By ([2], Propostion 3.2), $Z_{\mathrm{Bun}_{M}}^{\theta} \subset \widetilde{\operatorname{Bun}_{P}} \times_{\mathrm{Bun}_{G}} \operatorname{Bun}_{P^{-}}$is open. This extends naturally to an open immersion

$$
\widetilde{Z}_{\widetilde{\operatorname{Bun}}_{M}}^{\theta} \hookrightarrow{\widetilde{\operatorname{Bun}_{\tilde{P}}}}_{\tilde{B u n}_{G}} \operatorname{Bun}_{P^{-}}
$$

The restriction of $\mathrm{pr}_{1}^{*}\left(\mathrm{IC}_{\zeta}\right)\left[\operatorname{dim} . \mathrm{rel}\left(\mathrm{pr}_{1}\right)\right]$ under this open immersion identifies with (29) over the intersection with

$$
\widetilde{\operatorname{Bun}}_{\tilde{P}} \times_{\operatorname{Bun}_{G}} \operatorname{Bun}_{P^{-}}^{r}
$$

So, as in [2], $\mathrm{IC}_{Z^{\theta}, \zeta}$ is a local model for $\mathrm{IC}_{\zeta}$. 
5.1.3. The natural extensions of $\pi_{P}$ and $\mathfrak{s}^{\theta}$ are still denoted $\mathfrak{s}^{\theta}: \operatorname{Mod}_{\tilde{M}}^{+, \theta} \rightarrow \widetilde{Z}^{\theta}$ and $\pi_{P}$ : $\widetilde{Z}^{\theta} \rightarrow \operatorname{Mod}_{\tilde{M}}^{+, \theta}$.

If we pick a $\mathbb{G}_{m}$-action on $Z^{\theta}$ as in ([2], Section 5.3) then the line bundle $\mathcal{L}_{M}$ and its trivialization over $Z_{\max }^{\theta}$ are $\mathbb{G}_{m^{-}}$-equivariant, as $\mathbb{G}_{m}$ is a subgroup in $M_{X^{\theta}}$. So, $\mathrm{IC}_{Z^{\theta}, \zeta}$ is $\mathbb{G}_{m^{-}}$ equivariant, and the analog of ([2], Proposition 5.2) holds, there is a canonical isomorphism

$$
\mathfrak{s}^{\theta !}\left(\mathrm{IC}_{Z^{\theta}, \zeta}\right) \widetilde{\rightrightarrows} \pi_{P !}\left(\mathrm{IC}_{Z^{\theta}, \zeta}\right)
$$

over $\operatorname{Mod}_{\tilde{M}}^{+, \theta}$. Since all our objects are already defined over a suitable finite subfield of $k$, the analog of ([2], Corollary 5.5) holds, the latter complex is a direct sum of shifted perverse sheaves.

Recall that for a fixed $x \in X$ one denotes by $\mathbb{S}^{\theta}$ the preimage of Spec $k \stackrel{x}{\rightarrow} X \rightarrow X^{\theta}$ under $\pi_{G}: Z^{\theta} \rightarrow X^{\theta}$. The corresponding preimage under $\pi_{G}: \widetilde{Z}^{\theta} \rightarrow X^{\theta}$ is denoted $\widetilde{\mathbb{S}}^{\theta}$.

For $\theta^{\prime} \in \Lambda_{G, P}^{\text {pos }}$ with $\theta-\theta^{\prime} \in \Lambda_{G, P}^{\text {pos }}$ one has the locally closed subschemes $\theta^{\prime} Z^{\theta} \hookrightarrow Z^{\theta}$ and ${ }_{\theta^{\prime}} \mathbb{S}^{\theta} \hookrightarrow \mathbb{S}^{\theta}$ defined in ([2], Section 3.5). Restricting the gerb $\tilde{Z}^{\theta}$, one gets the locally closed substacks ${ }_{\theta^{\prime}} \widetilde{Z}^{\theta} \hookrightarrow \widetilde{Z}^{\theta}$ and ${ }_{\theta^{\prime}} \widetilde{\mathbb{S}}^{\theta} \hookrightarrow \widetilde{\mathbb{S}}^{\theta}$.

5.1.4. Recall that $\mathrm{Gr}_{M}^{\theta}$ is the connected component containing $t^{\nu} M(\mathbf{O})$ for any $\nu \in \Lambda$ over $\theta$. By virtue of ([2], Propostion 2.6) for $\theta \in \Lambda_{G, P}^{\text {pos }}$ the isomorphism $i_{\text {max }}$ restricts to an isomorphism

$$
i_{\text {max }}^{x}: B\left(\mu_{N}\right) \times \operatorname{Gr}_{P}^{\theta} \cap \mathrm{Gr}_{U\left(P^{-}\right)} \widetilde{\rightarrow}_{0} \widetilde{\mathbb{S}}^{\theta}
$$

sending $\mathcal{U}_{0}, \mathcal{U}_{0}^{N} \underset{\rightarrow}{\rightarrow},\left(\mathcal{F}_{G}, \mathcal{F}_{M}, \beta, \beta_{M}\right)$ to $\left(\mathcal{F}_{G}, \mathcal{F}_{M}, \beta, \beta_{M}, \mathcal{U}\right)$, where $\mathcal{U}=\mathcal{U}_{0}^{-1}$ is equipped with the induced isomorphism (28). The map $\pi_{P}$ restricts to a morphism

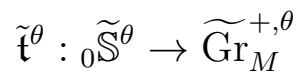

sending $\left(\mathcal{F}_{G}, \mathcal{F}_{M}, \beta, \beta_{M}, \mathcal{U}\right)$ to $\left(\mathcal{F}_{M}, \beta_{M}, \mathcal{U}\right)$. First, we prove the following analog of ([2], Theorem 5.9).

Theorem 5.1. 1) For $\theta \in \Lambda_{G, P}^{\text {pos }}$ the complex

$$
\tilde{\mathfrak{t}}_{!}^{\theta}\left(i_{\max }^{x}\right)_{*}\left(\mathcal{L}_{\zeta} \otimes \overline{\mathbb{Q}}_{\ell}\right)
$$

is placed in perverse degrees $\leq\left\langle\theta, 2\left(\check{\rho}-\check{\rho}_{M}\right)\right\rangle$.

2) Its $\left\langle\theta, 2\left(\check{\rho}-\check{\rho}_{M}\right)\right\rangle$-th perverse cohomology sheaf vanishes unless $\theta \in \Lambda_{G, P}^{\sharp}$. In the latter case it identifies canonically with $\operatorname{Loc}_{\zeta^{-1}}\left(U\left(\check{\mathfrak{u}}_{n}(P)\right)_{\theta}\right)$.

The map $\tilde{\mathfrak{t}}^{\theta}$ is $M\left(\mathbf{O}_{x}\right)$-equivariant, so each perverse cohomology sheaf of (30) is of the form $\operatorname{Loc}_{\zeta^{-1}}(V)$ for some $V \in \operatorname{Rep}\left(\check{M}_{n}\right)$.

5.1.5. Proof of Theorem 5.1 for $P=B$. By ([2], Section 6.3), for any $\nu \in \Lambda$ one has $\operatorname{dim}\left(\mathrm{Gr}_{B}^{\nu} \times \mathrm{Gr}_{U\left(B^{-}\right)}\right) \leq\langle\nu, \check{\rho}\rangle$. This implies part 1). Moreover, (30) vanishes unless $\theta \in \Lambda^{\sharp}$ because of the description of $\operatorname{Perv}_{T, G, n}$. 
Assume $\mu \in \Lambda^{+}$is deep enough in the dominant chamber so that $\mathrm{Gr}_{B}^{\nu-\mu} \cap \mathrm{Gr}_{B^{-}}^{-\mu} \subset \mathrm{Gr}_{G}^{-w_{0}(\mu)}$ by ([2], Proposition 6.4). By loc.cit., the inclusion

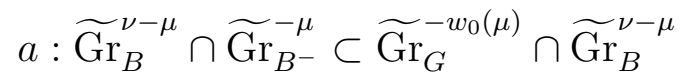

yields a bijection between the irreducible components of dimension $\langle\nu, \check{\rho}\rangle$ of both schemes. Recall that the multiplication by $t^{\mu}$ gives an isomorphism

$$
\mathrm{Gr}_{B}^{\nu-\mu} \cap \mathrm{Gr}_{B^{-}}^{-\mu} \widetilde{\rightarrow} \mathrm{Gr}_{B}^{\nu} \cap \mathrm{Gr}_{B^{-}}^{0}
$$

Recall that $\operatorname{det}\left(\mathfrak{g}(\mathbf{O}): \mathfrak{g}(\mathbf{O})^{t_{x}^{\mu}}\right) \underset{\rightarrow}{\rightarrow} \Omega_{\bar{c}}^{\breve{h}_{\iota}(\mu, \mu)}$. Assume in addition that $\mu \in 2 \Lambda^{\sharp}$. In this case we get a $T(\mathbf{O})$-equivariant diagram

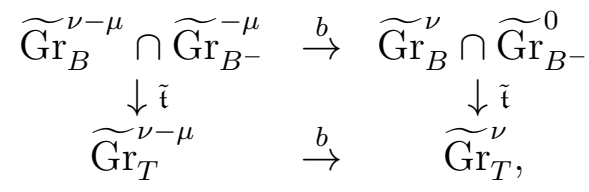

where for the top row $b$ is the isomorphism sending $\left(\mathcal{U}, \mathcal{U}^{N} \underset{\rightarrow}{\rightarrow} \operatorname{det}\left(\mathfrak{g}\left(\mathbf{O}_{x}\right): \mathfrak{g}(\mathbf{O})^{g}\right), g G\left(\mathbf{O}_{x}\right)\right)$ to $\left(\mathcal{U}^{\prime}, t^{\mu} g G\left(\mathbf{O}_{x}\right)\right)$, where $\mathcal{U}^{\prime}=\mathcal{U} \otimes \Omega_{\bar{c}}^{\frac{\imath(\mu, \mu)}{2 n}}$ is equipped with the induced isomorphism

$$
\mathcal{U}^{\prime N} \widetilde{\rightarrow} \operatorname{det}\left(\mathfrak{g}(\mathbf{O}): \mathfrak{g}(\mathbf{O})^{t_{x}^{\mu}}\right) \otimes \operatorname{det}\left(\mathfrak{g}(\mathbf{O}): \mathfrak{g}(\mathbf{O})^{g}\right) \widetilde{\rightarrow} \operatorname{det}\left(\mathfrak{g}(\mathbf{O}): \mathfrak{g}(\mathbf{O})^{t_{x}^{\mu} g}\right)
$$

For the low row $b$ is defined similarly. Using Lemma 5.1 below, one gets canonically

$$
\left(i_{\text {max }}^{x}\right)^{*} b_{*} a^{*} \mathcal{A}_{\varepsilon}^{-w_{0}(\mu)}[-\langle\mu, 2 \check{\rho}\rangle] \widetilde{\Im}\left(\mathcal{L}_{\zeta}^{*} \otimes \overline{\mathbb{Q}}_{\ell}\right)
$$

From Proposition 3.1 we see that

$$
\tilde{\mathfrak{t}}_{!}^{\nu-\mu}\left(\mathfrak{s}^{\nu-\mu}\right)^{*} \mathcal{A}_{\varepsilon}^{-w_{0}(\mu)}[-\langle\mu, 2 \check{\rho}\rangle]
$$

identifies with $\operatorname{Loc}\left(V^{-w_{0}(\mu)}(\nu-\mu)\right)[-\langle\nu, 2 \check{\rho}\rangle]$. If $\mu$ is large enough in the dominant chamber compared to $\nu$ then the latter identifies with $U\left(\check{\mathfrak{u}}_{n}\right)_{\nu}[-\langle\nu, 2 \check{\rho}\rangle]$. Here $V^{\mu_{1}}\left(\mu_{2}\right)$ denotes $\mu_{2^{-}}$ weight space of $\check{T}^{\sharp}$ in the irreducible representation $V^{\mu_{1}}$ of $\check{G}_{n}$ with highest weight $\mu_{1}$.

5.1.6. Proof of Theorem 5.1 for general $P$. Let $\theta \in \Lambda_{G, P}^{\text {pos }}$. For $\nu \in \Lambda$ dominant for $M$ with

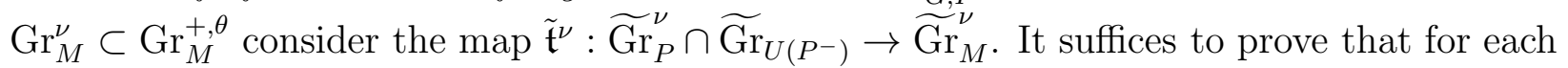
such $\nu$ the complex

$$
\left.\tilde{\mathfrak{t}}_{!}^{\nu}\left(i_{\text {max }}^{x}\right)_{*}\left(\mathcal{L}_{\zeta} \otimes \overline{\mathbb{Q}}_{\ell}\right)\right|_{\left.\widetilde{G r}_{P}^{\nu} \cap \widetilde{\mathrm{Gr}}_{U\left(P^{-}\right.}\right)}
$$

is placed in perverse degrees $\leq\left\langle\nu, 2 \check{\rho}-2 \check{\rho}_{M}\right\rangle$, and its $\left\langle\nu, 2 \check{\rho}-2 \check{\rho}_{M}\right\rangle$-th perverse cohomology sheaf vanishes unless $\nu \in \Lambda_{M}^{\sharp,+}$, and in the latter case identifies with

$$
\mathcal{A}_{M, \mathcal{E}, \zeta^{-1}}^{\nu} \otimes \operatorname{Hom}_{\check{M}_{n}}\left(U^{\nu}, U\left(\check{\mathfrak{u}}_{n}(P)\right)\right)
$$

Pick $\nu \in \Lambda$ dominant for $M$ with $\operatorname{Gr}_{M}^{\nu} \subset \mathrm{Gr}_{M}^{+, \theta}$. We have a diagram

$$
\begin{aligned}
& {\widetilde{\mathrm{Gr}_{P}}}_{P}^{\theta} \cap{\widetilde{\mathrm{Gr}_{U(P}}}_{U} \stackrel{\tilde{\mathfrak{t}}^{\theta}}{\rightarrow}{\widetilde{\mathrm{Gr}_{M}}}_{M}^{+, \theta} \\
& \uparrow \quad \uparrow
\end{aligned}
$$

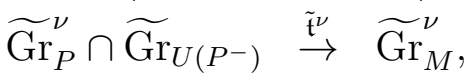


where vertical arrows are natural closed immersions. Let $Z(M)^{0}$ be the connected component of unity of the center $Z(M)$ of $M$. Pick $\mu \in \Lambda$ satisfying $\left\langle\mu, \check{\alpha}_{i}\right\rangle=0$ for $i \in \mathcal{J}_{M}$, and $\left\langle\mu, \check{\alpha}_{i}\right\rangle$ positive and large enough compared to $\nu$ for $i \in \mathcal{J}-\mathcal{J}_{M}$. So, $\mu \in \operatorname{Hom}\left(\mathbb{G}_{m}, Z(M)^{0}\right)$. The multiplication by $t^{\mu}$ yields a diagram, where the horizontal arrows are isomorphisms

$$
\begin{array}{cccc}
\operatorname{Gr}_{P}^{\nu-\mu} \cap \mathrm{Gr}_{P^{-}}^{-\mu} & \cong & \operatorname{Gr}_{P}^{\nu} \cap \mathrm{Gr}_{U\left(P^{-}\right)} \\
\downarrow \mathfrak{t}^{\nu-\mu} & & & \downarrow \mathfrak{t}^{\nu} \\
\operatorname{Gr}_{M}^{\nu-\mu} & \cong & \operatorname{Gr}_{M}^{\nu}
\end{array}
$$

We may and do assume by ([2], Proposition 6.6) that $\operatorname{Gr}_{P}^{\nu-\mu} \cap \mathrm{Gr}_{P^{-}}^{-\mu} \subset \mathrm{Gr}_{G}^{-w_{0}(\mu)}$.

Assume in addition that $\mu \in 2 \Lambda^{\sharp}$. Then (31) extends to a diagram of $M(\mathbf{O})$-equivariant morphisms

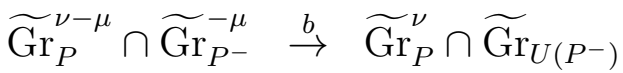

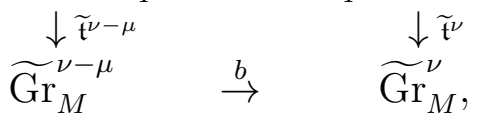

where for the top row $b$ is the isomorphism sending $\left(\mathcal{U}, \mathcal{U}^{N} \widetilde{\Im} \operatorname{det}\left(\mathfrak{g}\left(\mathbf{O}_{x}\right): \mathfrak{g}(\mathbf{O})^{g}\right), g G\left(\mathbf{O}_{x}\right)\right)$ to $\left(\mathcal{U}^{\prime}, t^{\mu} g G\left(\mathbf{O}_{x}\right)\right)$, where $\mathcal{U}^{\prime}=\mathcal{U} \otimes \Omega_{\bar{c}}^{\frac{\iota(\mu, \mu)}{2 n}}$ is equipped with the induced isomorphism

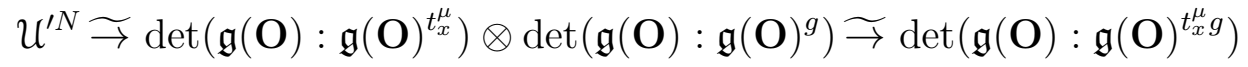

For the low row $b$ is defined similarly.

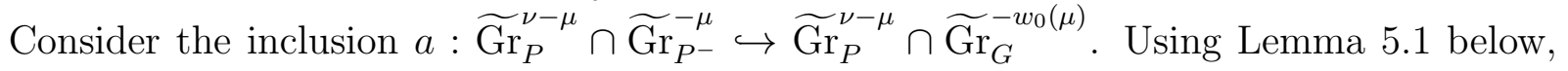
one gets a canonical isomorphism

$$
b_{*} a^{*} \mathcal{A}_{\mathcal{\varepsilon}}^{-w_{0}(\mu)}[-\langle\mu, 2 \check{\rho}\rangle] \widetilde{\rightrightarrows}\left(i_{\max }^{x}\right)_{*}\left(\mathcal{L}_{\zeta}^{*} \otimes \overline{\mathbb{Q}}_{\ell}\right)
$$

By ([3], Proposition 4.3.3), the fibres of the left vertical arrow $\tilde{\mathfrak{t}}^{\nu-\mu}$ in (32) are of dimension $\leq\left\langle\nu, \check{\rho}-2 \check{\rho}_{M}\right\rangle$, so $\tilde{\mathfrak{t}}_{!}^{\nu-\mu}\left(a^{*} E_{\mathcal{E}}^{-w_{0}(\mu)}\right)$ is placed in usual cohomological degrees $\leq\left\langle\nu, 2 \check{\rho}-4 \check{\rho}_{M}\right\rangle$, and this complex is $M(\mathbf{O})$-equivariant. So, it is placed in perverse degrees $\leq\left\langle\nu, 2 \check{\rho}-2 \check{\rho}_{M}\right\rangle$. The natural map

$$
\tilde{\mathfrak{t}}_{!}^{\nu-\mu}\left(E_{\mathcal{E}}^{-w_{0}(\mu)}\right) \rightarrow \tilde{\mathfrak{t}}_{!}^{\nu-\mu}\left(a_{*} a^{*} E_{\mathcal{E}}^{-w_{0}(\mu)}\right)
$$

induces an isomorphism between the corresponding $\left\langle\nu, 2 \check{\rho}-2 \check{\rho}_{M}\right\rangle$-th perverse cohomology sheaves over $\widetilde{G r}_{M}^{\nu-\mu}$, which identify by Corollary 4.1 with $\mathcal{A}_{M, \mathcal{\varepsilon}}^{\nu-\mu} \otimes \operatorname{Hom}_{\check{M}_{n}}\left(U^{\nu-\mu}, V^{-w_{0}(\mu)}\right)$ for $\nu \in \Lambda_{M}^{\sharp,+}$ and vanish otherwise. Assume $\nu \in \Lambda_{M}^{\sharp,+}$. Since $\mu$ is large enough on the corresponding wall of the Weyl chamber compared to $\nu$, we have

$$
\operatorname{Hom}_{\check{M}_{n}}\left(U^{\nu-\mu}, V^{-w_{0}(\mu)}\right) \widetilde{\rightarrow} \operatorname{Hom}_{\check{M}_{n}}\left(U^{\nu}, U\left(\check{\mathfrak{u}}_{n}(P)\right)\right)
$$

Theorem 5.1 is proved.

For $\mu \in \Lambda^{\sharp,+}$ we have the line bundle $\mathcal{L}_{\mu, \mathcal{E}}$ on $\mathrm{Gr}_{G}^{\mu}$ defined in ([9], Section 2.1, p. 723). Its analog for the Levi $M$ is the following line bundle $\mathcal{L}_{\mu, M, \mathcal{\varepsilon}}$. For $\mu \in \Lambda_{M}^{\sharp,+}$ let $\mathcal{B}_{M}^{\mu}=M / P_{M}^{\mu}$ be 
the $M$-orbit through $t^{\mu} M(\mathbf{O})$ in $\operatorname{Gr}_{M}$ as in ([9], Section 4.1.1). Denote by $\tilde{\omega}_{M, \mu}: \operatorname{Gr}_{M}^{\mu} \rightarrow \mathcal{B}_{M}^{\mu}$ the projection. Set

$$
\mathcal{L}_{\mu, M, \mathcal{E}}=\mathcal{E}_{\bar{c}}^{\iota(\mu, \mu) / n} \otimes \tilde{\omega}_{M, \mu} \mathcal{O}(\iota(\mu) / n)
$$

Note that for $\mu \in 2 \Lambda^{\sharp}$ it does not depend on $\mathcal{E}$. Over $\operatorname{Gr}_{M}^{\mu}$ one has the isomorphism

$$
\left.\mathcal{L}\right|_{\mathrm{Gr}_{M}^{\mu}} \widetilde{\rightarrow} \mathcal{L}_{\mu, M, \mathcal{E}}^{N}
$$

Lemma 5.1. Let $\mu \in 2 \Lambda^{\sharp,+}$ be orthogonal to all roots of $M$. Consider the map $\mathfrak{t}^{-\mu}: \mathrm{Gr}_{P^{-}}^{-\mu} \rightarrow$ $\mathrm{Gr}_{M}^{-\mu}$. There is a natural isomorphism

$$
\left.\left(\left(\mathfrak{t}_{P^{-}}^{-\mu}\right)^{*} \mathcal{L}_{-\mu, M, \varepsilon}\right)\right|_{\mathrm{Gr}_{P^{-}}^{-\mu} \cap \mathrm{Gr}_{G}^{-w_{0}(\mu)}} \stackrel{\widetilde{T}}{\mathcal{L}}-w_{0}(\mu),\left.\mathcal{E}\right|_{\mathrm{Gr}_{P^{-}}^{-\mu} \cap \mathrm{Gr}_{G}^{-w_{0}(\mu)}}
$$

compatible with the isomorphisms (33) for $M$ and $G$.

Proof The intersection of $\mathcal{B}_{G}^{-w_{0}(\mu)} \cap \mathrm{Gr}_{P^{-}}^{-\mu}$ is the point $t^{-\mu} G(\mathbf{O})$ fixed by $M$. So, over $\mathrm{Gr}_{P^{-}}^{-\mu} \cap \mathrm{Gr}_{G}^{-w_{0}(\mu)}$ both line bundles in (34) are constant, and it suffices to establish the desired isomorphism at the point $t^{-\mu} G(\mathbf{O})$. The fibres of both line bundles at this point identify with $\mathcal{E}_{\bar{c}}^{\iota(\mu, \mu) / n}$ in a way compatible with (33) for $M$ and $G$.

5.2. Main technical step. The purpose of this section is to formulate Theorem 5.2, which is an analog of ([2], Theorem 4.5) and the main technical step in the proof of Theorem 4.1.

Define $\Lambda_{G, P}^{\text {pos, pos }}$ as the free abelian semigroup with base $J$. Recall the map $c_{P}$ from Section 4.3. Let

$$
\bar{c}_{P}: \Lambda_{G, P}^{\text {pos, pos }} \rightarrow \Lambda_{G, P}^{\sharp, p o s}
$$

be the morphism of semigroups, which on the base of $\Lambda_{G, P}^{\text {pos, pos }}$ is given by $c_{P}$. For $\theta \in \Lambda_{G, P}^{\sharp, p o s}$ we will denote by $\mathfrak{B}(\theta)$ the elements of $\Lambda_{G, P}^{\text {pos,pos }}$ sent by $\bar{c}_{P}$ to $\theta$.

Let $\theta \in \Lambda_{G, P}^{\sharp, p o s}$. Let $\mathfrak{B}(\theta)=\sum_{\nu \in J} n_{\nu} \nu$ be an element of $\Lambda_{G, P}^{\text {pos,pos }}$ over $\theta$. Set $|\mathfrak{B}(\theta)|=\sum_{\nu} n_{\nu}$. Write $X^{\mathfrak{B}(\theta)}$ for the moduli scheme of $\Lambda_{G, P}^{\text {pos,pos }}$-valued divisors of degree $\mathfrak{B}(\theta)$, so

$$
X^{\mathfrak{B}(\theta)} \underset{\rightarrow}{\rightarrow} \prod_{\nu \in J} X^{\left(n_{\nu}\right)}
$$

To a point $\left(D_{\nu}, \nu \in J\right)$ of the latter scheme there corresponds the divisor $D=\sum_{\nu \in J} D_{\nu} \nu$. The map $\bar{c}_{P}$ yields a finite morphism $X^{\mathfrak{B}(\theta)} \rightarrow X^{\theta}$. Let $\stackrel{\circ}{X^{\mathfrak{B}(\theta)}} \subset X^{\mathfrak{B}(\theta)}$ be the complement to all the diagonals.

If $D=\sum_{k} x_{k} \theta_{k} \in X^{\theta}$ and $x_{k}$ are pairwise different then the fibre of $\operatorname{Mod}_{M}^{+, \theta} \rightarrow X^{\theta}$ over $D$ is $\prod_{k} \operatorname{Gr}_{M}^{+, \theta_{k}}$. Let $\mathrm{IC}_{\zeta}^{\mathfrak{B}(\theta), 0}$ be the perverse sheaf on $\stackrel{\circ}{X^{\mathfrak{B}}(\theta)} \times_{X^{\theta}} \operatorname{Mod}_{\tilde{M}}^{+, \theta}$ on which $\mu_{N}(k)$ acts by $\zeta^{-1}$ and such that for $D=\sum_{k} x_{k} \nu_{k} \in \stackrel{\circ}{X^{\mathfrak{B}}(\theta)}$ with $\theta_{k}=c_{P}\left(\nu_{k}\right)$ its restriction to

$$
\prod_{k}{\widetilde{\mathrm{Gr}_{M, x_{k}}}}^{+, \theta_{k}}
$$


is $\bigotimes_{k} \operatorname{Loc}_{\zeta^{-1}}\left(\left(\check{\mathfrak{u}}_{n}(P)\right)_{\nu_{k}}\right)[|\mathfrak{B}(\theta)|]$. One makes this definition rigorous as in Section 4.5.1, This perverse sheaf is defined up to a unique isomorphism and irreducible. Let $\mathrm{IC}_{\zeta}^{\mathfrak{B}(\theta)}$ be the intermediate extension of $\mathrm{IC}_{\zeta}^{\mathfrak{B}(\theta), 0}$ under

$$
\stackrel{\circ}{X}^{\mathfrak{B}(\theta)} \times_{X^{\theta}} \operatorname{Mod}_{\tilde{M}}^{+, \theta} \hookrightarrow X^{\mathfrak{B}(\theta)} \times_{X^{\theta}} \operatorname{Mod}_{\tilde{M}}^{+, \theta}
$$

Denote by $i_{\mathfrak{B}(\theta)}: X^{\mathfrak{B}(\theta)} \times_{X^{\theta}} \operatorname{Mod}_{\tilde{M}}^{+, \theta} \rightarrow \operatorname{Mod}_{\tilde{M}}^{+, \theta}$ the second projection.

For $\nu \in J$ let $\theta_{\nu}=c_{P}(\nu)$. We get a decomposition $\mathfrak{U}(\theta)$ given by $\theta=\sum_{\nu \in J} n_{\nu} \theta_{\nu}$, and $X^{\mathfrak{B}(\theta)} \underset{\rightarrow}{\rightarrow} X^{\mathfrak{U}(\theta)}$ naturally. It follows that $i_{\mathfrak{B}(\theta) *}\left(\mathrm{IC}_{\zeta}^{\mathfrak{B}(\theta)}\right)$ is the intermediate extension from

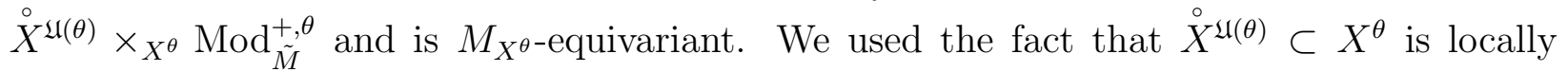
closed.

Theorem 5.2. Let $\theta \in \Lambda_{G, P}^{\text {pos }}$. For the $\operatorname{map} \mathfrak{s}^{\theta}: \operatorname{Mod}_{\tilde{M}}^{+, \theta} \rightarrow \widetilde{Z}^{\theta}$ there is a $M_{X^{\theta} \text {-equivariant }}$ isomorphism

$$
\mathfrak{s}^{\theta !}\left(\mathrm{IC}_{Z^{\theta}, \zeta}\right) \stackrel{\rightrightarrows}{\rightarrow} \underset{\mathfrak{B}(\theta)}{\oplus} i_{\mathfrak{B}(\theta) *}\left(\mathrm{IC}_{\zeta}^{\mathfrak{B}(\theta)}\right)[-|\mathfrak{B}(\theta)|]
$$

In particular, this complex vanishes unless $\theta \in \Lambda_{G, P}^{\sharp, p o s}$.

The following is proved exactly as ([2], Lemma 4.3).

Lemma 5.2. The $*$-restriction of $\mathrm{IC}_{\zeta}^{\mathfrak{B}(\theta)}$ to $X \times_{X^{\theta}} \operatorname{Mod}_{\tilde{M}}^{+, \theta} \widetilde{\rightarrow} \widetilde{\mathrm{Gr}}_{M, X}^{+, \theta}$ identifies canonically with

$$
\operatorname{Loc}_{X, \zeta^{-1}}\left(\otimes_{\nu \in J} \operatorname{Sym}^{n_{\nu}}\left(\check{\mathfrak{u}}_{n}(P)_{\nu}\right)\right)[-1+|\mathfrak{B}(\theta)|]
$$

The functor $\operatorname{Loc}_{X}=\left(\tau^{0} \mathrm{Loc}\right)[1]$ used in Lemma 5.2 takes values in $M_{X}$-equivariant perverse sheaves on $\widetilde{\mathrm{Gr}}_{M, X}$.

5.2.1. Let $\theta \in \Lambda_{G, P}^{\text {pos }}$. Recall that $\operatorname{Mod}_{\mathrm{Bun}_{M}}^{+, \theta}$ classifies $D \in X^{\theta}, \mathcal{F}_{M}, \mathcal{F}_{M}^{\prime} \in \operatorname{Bun}_{M}$ and an isomorphism $\beta_{M}:\left.\mathcal{F}_{M} \widetilde{\rightarrow} \mathcal{F}_{M}^{\prime}\right|_{X-D}$ such that for each $\mathcal{V} \in \operatorname{Rep}(G)$ the map $\beta_{M}: \mathcal{V}_{\mathcal{F}_{M}}^{U(P)} \rightarrow$ $\mathcal{V}_{\mathcal{F}_{M}^{\prime}}^{U(P)}$ is regular, and $\beta_{M}$ induces an isomorphism

$$
\mathcal{F}_{M /[M, M]} \stackrel{\rightarrow}{\rightarrow} \mathcal{F}_{M /[M, M]}^{\prime}(-D)
$$

Consider the projection $\operatorname{Mod}_{\mathrm{Bun}_{M}}^{+, \theta} \rightarrow \operatorname{Bun}_{M} \times \operatorname{Bun}_{M}$ sending the above point to $\left(\mathcal{F}_{M}, \mathcal{F}_{M}^{\prime}\right)$.

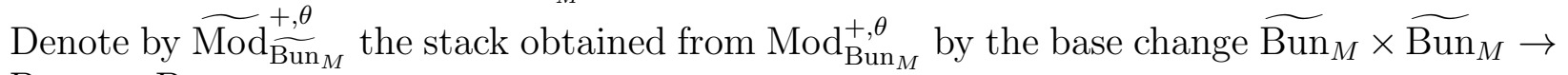
$\operatorname{Bun}_{M} \times \operatorname{Bun}_{M}$.

Recall the stacks $\operatorname{Bun}_{M, X^{\theta}}$ and $\widetilde{\operatorname{Bun}}_{M, X^{\theta}}$ from Section 5.1.2. As we have seen in Section 5.1.1, $M_{X^{\theta}}$ acts on $\operatorname{Mod}_{M}^{+, \theta}$ over $X^{\theta}$. Let $M_{X^{\theta}}$ act diagonally on $\operatorname{Bun}_{M, X^{\theta}} \times_{X^{\theta}} \operatorname{Mod}_{M}^{+, \theta}$, the corresponding stack quotient is denoted $\operatorname{Bun}_{M, X^{\theta}} \times_{M_{X^{\theta}}} \operatorname{Mod}_{M}^{+, \theta}$. Let

$$
\gamma \rightarrow \operatorname{Bun}_{M, X^{\theta}} \times_{M_{X^{\theta}}} \operatorname{Mod}_{M}^{+, \theta} \underset{\rightarrow}{\rightarrow} \operatorname{Mod}_{\operatorname{Bun}_{M}}^{+, \theta}
$$


be the natural isomorphism such that the projection to the first factor corresponds to $h_{M}$. As in Section 4.5.1, we get a $M_{X^{\theta}}$-torsor

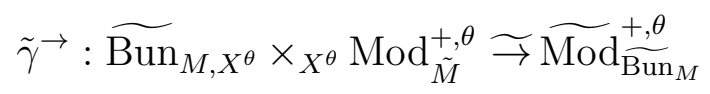

This allows to introduce for each $\mathfrak{B}(\theta)$ the relative version $\mathrm{IC}_{\mathrm{Bun}}^{\mathfrak{B}(\theta)}, \zeta$ of $\operatorname{IC}_{\zeta}^{\mathfrak{B}(\theta)}$, which is a

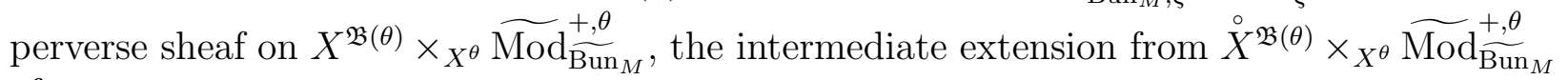
of

$$
\left(\mathrm{IC}\left(\widetilde{\operatorname{Bun}}_{M}\right) \tilde{\otimes} \mathrm{IC}_{\zeta}^{\mathfrak{B}(\theta), 0}\right)^{r}
$$

The latter is the descent of $\operatorname{IC}\left(\widetilde{\operatorname{Bun}}_{M, X^{\theta}}\right) \otimes \mathrm{IC}_{\zeta}^{\mathfrak{B}(\theta), 0}\left[-\operatorname{dim} X^{\theta}\right]$ under the $\stackrel{\circ}{X}^{\mathfrak{B}(\theta)} \times_{X^{\theta}} M_{X^{\theta}}$-torsor

$$
\tilde{\gamma} \rightarrow: \widetilde{\operatorname{Bun}}_{M, X^{\theta}} \times_{X^{\theta}} \stackrel{\circ}{X}^{\mathfrak{B}(\theta)} \times_{X^{\theta}} \operatorname{Mod}_{\tilde{M}}^{+, \theta} \rightarrow \stackrel{\circ}{X^{\mathfrak{B}(\theta)}} \times_{X^{\theta}} \widetilde{\operatorname{Mod}_{\widetilde{\operatorname{Bun}}_{M}}^{+, \theta}}
$$

Write also $\nu_{P}$ for the projection $\operatorname{Bun}_{P} \times_{\operatorname{Bun}_{M}} \widetilde{\operatorname{Mod}_{\operatorname{Bun}_{M}}^{+, \theta}} \rightarrow \widetilde{\operatorname{Mod}}+\frac{+, \theta}{\operatorname{Bun}_{M}}$, where we used $h_{M}$ to define the corresponding fibred product. We have the locally closed embedding

$$
\operatorname{Bun}_{P} \times_{\operatorname{Bun}_{M}} \widetilde{\operatorname{Mod}_{\operatorname{Bun}_{M}}^{+, \theta}} \hookrightarrow \widetilde{\operatorname{Bun}_{\tilde{P}}}
$$

sending $\left(D, \mathcal{F}_{M}, \mathcal{F}_{M}^{\prime}, \beta_{M}:\left.\mathcal{F}_{M} \widetilde{\rightarrow} \mathcal{F}_{M}^{\prime}\right|_{X-D}, \mathcal{U}, \mathcal{U}^{\prime}\right) \in \widetilde{\operatorname{Mod}_{\operatorname{Bun}_{M}}^{+, \theta}}, \mathcal{F}_{P} \in \operatorname{Bun}_{P}$ with $\mathcal{F}_{P}^{\prime} \times_{P}$ $M \widetilde{\Im} \mathcal{F}_{M}^{\prime}$ to $\left(\mathcal{F}_{G}^{\prime}, \mathcal{F}_{M}, \kappa, \mathcal{U}, \mathcal{U}^{\prime}\right)$, where $\mathcal{F}_{G}^{\prime}=\mathcal{F}_{P} \times_{P} G$. Its image is denoted ${ }_{\theta} \widetilde{\operatorname{Bun}}_{\tilde{P}}$.

Translating Theorem 5.2 to $\widetilde{\operatorname{Bun}}_{\tilde{P}}$ we obtain the following.

Corollary 5.1. The *-restriction of $\mathrm{IC}_{\zeta}$ to ${ }_{\theta} \widetilde{\operatorname{Bun}_{\tilde{P}}} \widetilde{\rightarrow} \operatorname{Bun}_{P} \times{ }_{\operatorname{Bun}_{M}} \widetilde{\operatorname{Mod}_{\operatorname{Bun}_{M}}^{+, \theta}}$ vanishes unless $\theta \in \Lambda_{G, P}^{\sharp, p o s}$. In the latter case it identifies with $\nu_{P}^{*}\left[\operatorname{dim} . \operatorname{rel}\left(\nu_{P}\right)\right]$ applied to the complex

$$
\underset{\mathfrak{B}(\theta)}{\oplus}\left(i_{\mathrm{Bun}_{M}, \mathfrak{B}(\theta)}\right)_{*} \mathrm{IC}_{\mathrm{Bun}_{M}, \zeta}^{\mathfrak{B}(\theta)}[|\mathfrak{B}(\theta)|]
$$

Here we denoted by $i_{\operatorname{Bun}_{M}, \mathfrak{B}(\theta)}: X^{\mathfrak{B}(\theta)} \times_{X^{\theta}} \widetilde{\operatorname{Mod}}_{\widetilde{\operatorname{Bun}}_{M}}^{+, \theta} \rightarrow \widetilde{\operatorname{Mod}_{\operatorname{Bun}_{M}}^{+, \theta}}$ the projection.

5.3. Proof of Theorem 4.1. As ([2], Theorem 1.12) it is derived from Corollary 5.1 and Lemma 5.2. The proof uses the following combinatorial identity. Given $\theta \in \Lambda_{G, P}^{\text {pos }}$ and $m \geq 0$ the space $\left(\operatorname{Sym}^{m} \mathfrak{u}_{n}(P)\right)_{\theta}$ vanishes unless $\theta \in \Lambda_{G, P}^{\sharp, p o s}$, and in the latter case

$$
\left(\operatorname{Sym}^{m} \mathfrak{u}_{n}(P)\right)_{\theta}=\sum_{\mathfrak{B}(\theta)=\sum_{\nu \in J} n_{\nu},|\mathfrak{B}(\theta)|=m}\left(\otimes_{\nu \in J} \operatorname{Sym}^{n_{\nu}}\left(\check{\mathfrak{u}}_{n}(P)_{\nu}\right)\right)
$$

This implies

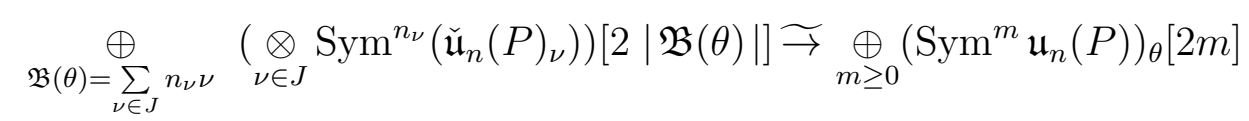

Theorem 4.1 is proved (modulo Theorem [5.2). 
5.4. Induction. Now we prove Theorem 5.2 by induction on $\theta$. Recall that for $\theta=\theta_{1}+\theta_{2}$ with $\theta_{i} \in \Lambda_{G, P}^{\text {pos }}$ the factorization property yields a natural $\mu_{N}(k)$-gerb

$$
\widetilde{Z}^{\theta_{1}} \times \widetilde{Z}^{\theta_{2}} \times{ }_{\left(X^{\theta_{1}} \times X^{\theta_{2}}\right)}\left(X^{\theta_{1}} \times X^{\theta_{2}}\right)_{d i s j} \rightarrow \widetilde{Z}^{\theta} \times_{X^{\theta}}\left(X^{\theta_{1}} \times X^{\theta_{2}}\right)_{d i s j},
$$

and the restriction of $\mathrm{IC}_{Z^{\theta}, \zeta}$ under this map is canonically identified with $\mathrm{IC}_{Z^{\theta_{1}, \zeta}} \otimes \mathrm{IC}_{Z^{\theta_{2}, \zeta}}$. Similarly, we have

$\sqcup_{\mathfrak{B}\left(\theta_{1}\right), \mathfrak{B}\left(\theta_{2}\right)}\left(X^{\mathfrak{B}\left(\theta_{1}\right)} \times X^{\mathfrak{B}\left(\theta_{2}\right)}\right) \times_{X^{\theta_{1}} \times X^{\theta_{2}}}\left(X^{\theta_{1}} \times X^{\theta_{2}}\right)_{d i s j} \stackrel{\sim}{\rightarrow} \sqcup_{\mathfrak{B}(\theta)} \quad X^{\mathfrak{B}(\theta)} \times_{X^{\theta}}\left(X^{\theta_{1}} \times X^{\theta_{2}}\right)_{\text {disj }}$

and the perverse sheaves $\mathrm{IC}_{\zeta}^{\mathfrak{B}(\theta)}$ also naturally factorise. So, by the induction hypothesis locally over $X^{\theta}-\triangle_{X}$ we get an isomorphism

$$
\mathfrak{s}^{\theta !}\left(\mathrm{IC}_{Z^{\theta}, \zeta}\right) \stackrel{\Im}{\rightarrow} \underset{\mathfrak{B}(\theta),|\mathfrak{B}(\theta)| \neq 1}{\oplus} i_{\mathfrak{B}(\theta) *}\left(\mathrm{IC}_{\zeta}^{\mathfrak{B}(\theta)}\right)[-|\mathfrak{B}(\theta)|]
$$

As in [2], globally we could have a nontrivial monodromy for $\beta(\theta)=2 \nu$ with $\nu \in J$. So, there is a rank one and order at most 2 local system $\mathcal{E}_{\mathfrak{B}(\theta)}$ on $X^{\mathfrak{B}(\theta)} \times_{X^{\theta}}\left(X^{\theta}-\triangle_{X}\right)$ and an isomorphism

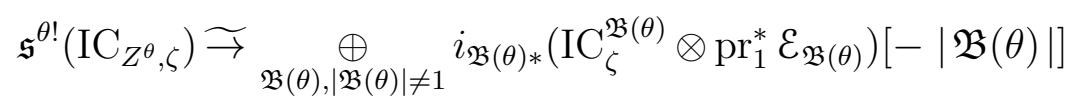

over $\operatorname{Mod}_{\tilde{M}}^{+, \theta} \times_{X^{\theta}}\left(X^{\theta}-\triangle_{X}\right)$.

Let $\mathcal{K}^{\mathfrak{B}(\theta)}$ be the intermediate extension of $\operatorname{IC}_{\zeta}^{\mathfrak{B}(\theta)} \otimes \operatorname{pr}_{1}^{*} \mathcal{E}_{\mathfrak{B}(\theta)}$ to $X^{\mathfrak{B}(\theta)} \times_{X^{\theta}} \operatorname{Mod}_{\tilde{M}}^{+, \theta}$. We get an isomorphism

$$
\mathfrak{s}^{\theta !}\left(\mathrm{IC}_{Z^{\theta}, \zeta}\right) \widetilde{\rightrightarrows} \underset{\mathfrak{B}(\theta),|\mathfrak{B}(\theta)| \neq 1}{\oplus} i_{\mathfrak{B}(\theta) *}\left(\mathcal{K}^{\mathfrak{B}(\theta)}\right)[-|\mathfrak{B}(\theta)|] \oplus \mathcal{K}^{\theta}
$$

where $\mathcal{K}^{\theta}$ is a pure complex supported over $\operatorname{Mod}_{\tilde{M}}^{+, \theta} \times_{X^{\theta}} X \widetilde{\Im} \widetilde{\mathrm{Gr}}_{M, X}^{+, \theta}$.

As in [2], let $\Delta_{x}$ denote the closed subscheme Spec $k \stackrel{x}{\rightarrow} X \stackrel{\Delta x}{\rightarrow} X^{\theta}$. The analogs of ([2], Proposition 5.7 and 5.8) are as follows.

Proposition 5.1. The complex $\left.\mathfrak{s}^{\theta !}\left(\mathrm{IC}_{Z^{\theta}, \zeta}\right)\right|_{\Delta x}$ over $\widetilde{\mathrm{Gr}}_{M}^{+, \theta}$ is placed in perverse degrees $\leq 0$. Its 0 -th perverse cohomology sheaf vanishes unless $\theta \in \Lambda_{G, P}^{\sharp}$, in the latter case it is identified with $\operatorname{Loc}_{\zeta^{-1}}\left(U\left(\check{\mathfrak{u}}_{n}(P)\right)_{\theta}\right)$.

Recall the map $\pi_{P}: \widetilde{Z}^{\theta} \rightarrow \operatorname{Mod}_{\tilde{M}}^{+, \theta}$.

Proposition 5.2. Assume $\theta^{\prime}, \theta-\theta^{\prime} \in \Lambda_{G, P}^{\text {pos }}$.

1) The complex $\pi_{P !}\left(\left.\mathrm{IC}_{Z^{\theta}, \zeta}\right|_{\theta^{\prime} \widetilde{\mathbb{S}}^{\theta}}\right)$ is placed in strictly negative perverse degrees for $\theta^{\prime} \neq 0$.

2) The complex $\pi_{P !}\left(\left.\mathrm{IC}_{Z^{\theta}, \zeta}\right|_{0} \widetilde{\mathbb{S}}^{\theta}\right)$ is placed in perverse degrees $\leq 0$.

3) The 0-th perverse cohomology sheaf of $\pi_{P !}\left(\left.\mathrm{IC}_{Z^{\theta}, \zeta}\right|_{0_{0} \widetilde{S}^{\theta}}\right)$ vanishes unless $\theta \in \Lambda_{G, P}^{\sharp}$, in the latter case it identifies with $\operatorname{Loc}_{\zeta^{-1}}\left(U\left(\check{\mathfrak{u}}_{n}(P)\right)_{\theta}\right)$. 
Let $\operatorname{Conv}_{M}$ denote the convolution diagram for the affine grassmanian of $M$ at $x$. This is the scheme classifying $\mathcal{F}_{M}, \mathcal{F}_{M}^{\prime} \in \operatorname{Bun}_{M}$ with isomorphisms $\tilde{\beta}_{M}:\left.\mathcal{F}_{M} \widetilde{\rightarrow}_{\mathcal{F}}^{\prime}\right|_{X-x}$ and $\beta_{M}^{\prime}:\left.\mathcal{F}_{M}^{\prime} \widetilde{\rightarrow} \mathcal{F}_{M}^{0}\right|_{X-x}$. Let $\widehat{\operatorname{Conv}}_{\tilde{M}}$ be obtained from $\operatorname{Conv}_{M}$ by adding two lines $\mathcal{U}, \mathcal{U}^{\prime}$ and isomorphisms

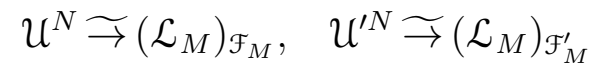

Write $\operatorname{pr}^{\prime}: \widetilde{\operatorname{Conv}}_{\tilde{M}} \rightarrow{\widetilde{\mathrm{Gr}_{M}}}$ for the projection sending the above point to $\left(\mathcal{F}_{M}^{\prime}, \beta_{M}^{\prime}, \mathcal{U}^{\prime}\right)$. It makes $\widetilde{\operatorname{Conv}}_{\tilde{M}}$ a fibration over $\widetilde{\mathrm{Gr}}_{M}$ with typical fibre isomorphic to $\widetilde{\mathrm{Gr}}_{M}$. Now given a $M\left(\mathbb{O}_{x}\right)$-equivariant perverse sheaf $\mathcal{S}$ on $\widetilde{\mathrm{Gr}}_{M}$ on which $\mu_{N}(k)$ acts as $\zeta^{-1}$, and any complex $\mathcal{S}^{\prime}$ on $\widetilde{\mathrm{Gr}}_{M}$, on which $\mu_{N}(k)$ acts as $\zeta^{-1}$, we can form their twisted external product $\mathcal{S} \tilde{\nabla} \mathcal{S}^{\prime}$, which is $\mathcal{S}^{\prime}$ along the base and $\mathcal{S}$ along the fibre. It is normalized to be perverse for $\mathcal{S}^{\prime}$ perverse. As in [11], one proves the following. Let pr : $\widetilde{\operatorname{Conv}}_{\tilde{M}} \rightarrow \widetilde{\operatorname{Gr}}_{M}$ be the map sending the above point to $\left(\mathcal{F}_{M}, \beta_{M}^{\prime} \tilde{\beta}_{M}, \mathcal{U}\right)$. The convolution of $\mathcal{S}$ with $\mathcal{S}^{\prime}$ is defined as $\operatorname{pr}_{!}\left(\mathcal{S} \tilde{\otimes} \mathcal{S}^{\prime}\right)$.

Lemma 5.3. If $\mathcal{S}$ is a $M\left(\mathbb{O}_{x}\right)$-equivariant perverse sheaf on $\widetilde{G r}_{M}$ on which $\mu_{N}(k)$ acts as $\zeta^{-1}, \mathcal{S}^{\prime}$ is a perverse sheaf on $\widetilde{\mathrm{Gr}}_{M}$, on which $\mu_{N}(k)$ acts as $\zeta^{-1}$ then their convolution is a perverse sheaf on $\widetilde{\mathrm{Gr}}_{M}$.

Proof of Proposition 5.2

As in the proof of ([2], Proposition 5.8), one has $\operatorname{dim} Z^{\theta}=\left\langle\theta, 2\left(\check{\rho}-\check{\rho}_{M}\right)\right\rangle$, and points 2),3) follow from Theorem 5.1.

Assume now $\theta^{\prime} \neq \theta$. As in ([2], Section 3.5), let $\operatorname{Conv}_{M}^{+, \theta^{\prime}}$ denote the closed subscheme of the convolution diagram $\operatorname{Conv}_{M}$ at $x$ given by the property $\left(\mathcal{F}_{M}, \mathcal{F}_{M}^{\prime}, \tilde{\beta}_{M}\right) \in \operatorname{Mod}_{\mathrm{Bun}_{M}}^{+, \theta^{\prime}}$. As in loc.cit., one has an isomorphism

$$
{ }_{0} \mathbb{S}^{\theta-\theta^{\prime}} \times{ }_{\mathrm{Gr}_{M}} \operatorname{Conv}_{M}^{+, \theta^{\prime}} \underset{\rightarrow}{\theta^{\prime}} \mathbb{S}^{\theta}
$$

here the map $\operatorname{Conv}_{M}^{+, \theta^{\prime}} \rightarrow \operatorname{Gr}_{M}$ used to define the fibred product sends the above point of $\operatorname{Conv}_{M}^{+, \theta^{\prime}}$ to $\left(\mathcal{F}_{M}^{\prime}, \beta_{M}^{\prime}\right)$. Let $\widetilde{\operatorname{Conv}}_{\tilde{M}}^{+, \theta^{\prime}}$ be obtained from $\operatorname{Conv}_{M}^{+, \theta^{\prime}}$ by the base change $\widetilde{\operatorname{Conv}}_{\tilde{M}} \rightarrow$ $\operatorname{Conv}_{M}$. We get a natural $\mu_{N-\text { gerb }}$

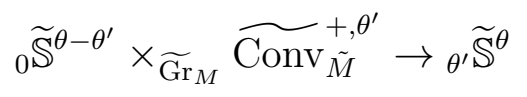

given by forgetting $\mathcal{U}^{\prime}$. As in the proof of ([2], Proposition 5.8), the *-restriction of $\left.\mathrm{IC}_{Z^{\theta}, \zeta}\right|_{\theta^{\prime}, \widetilde{\mathbb{S}}^{\theta}}$ under this gerb is described by the induction hypothesis. Namely, by Corollary 5.1 and Lemma 5.2, it identifies with

$$
\underset{\mathfrak{B}\left(\theta^{\prime}\right)}{\oplus} \operatorname{Loc}_{\zeta^{-1}}\left(\otimes_{\nu \in J} \operatorname{Sym}^{n_{\nu}}\left(\check{\mathfrak{u}}_{n}(P)_{\nu}\right)\left[2\left|\mathfrak{B}\left(\theta^{\prime}\right)\right|\right] \tilde{\otimes}\left(\left.\mathrm{IC}_{Z^{\theta-\theta^{\prime}, \zeta}}\right|_{0} \widetilde{\mathbb{S}}^{\theta-\theta^{\prime}}\right)\right.
$$

Here it is understood that $\mathfrak{B}\left(\theta^{\prime}\right)=\sum_{\nu \in J} n_{\nu} \nu$, the sum being taken over all elements $\mathfrak{B}\left(\theta^{\prime}\right)$ over $\theta^{\prime}$. Now by 2$), \pi_{P !}\left(\left.\mathrm{IC}_{Z^{\theta-\theta^{\prime}, \zeta}}\right|_{0_{0} \widetilde{\mathbb{S}}^{\theta-\theta^{\prime}}}\right)$ is placed in perverse degrees $\leq 0$. So, by Lemma 5.3 , $\pi_{P !}\left(\left.\mathrm{IC}_{Z^{\theta}, \zeta}\right|_{0} \widetilde{\mathbb{S}}^{\theta}\right)$ is placed in perverse degrees $<0$. 
In the case $\theta^{\prime}=\theta$ the complex $\pi_{P !}\left(\left.\mathrm{IC}_{Z^{\theta}, \zeta}\right|_{\theta^{\prime}, \widetilde{S}^{\theta}}\right)$ is placed in strictly negative perverse degrees, as $\pi_{P}:{ }_{\theta} \widetilde{\mathbb{S}}^{\theta} \rightarrow \widetilde{\mathrm{Gr}}_{M}^{+, \theta}$ is an isomorphism. We are done.

Now as in ([2], Section 5.11) one checks that all the local systems $\mathcal{E}_{\mathfrak{B}(\theta)}$ are trivial.

To finish the proof of Theorem 5.2 it remains to analyze the complex $\mathcal{K}^{\theta}$ from (35). There is at most one $\mathfrak{B}(\theta)$ with $|\mathfrak{B}(\theta)|=1$, which we denote $\mathfrak{B}(\theta)^{0}$ as in [2]. If it exists, that is, $\theta=c_{P}(\nu)$ for some $\nu \in J$, we have to show that $\mathcal{K}^{\theta} \widetilde{\rightrightarrows}\left(i_{\mathfrak{B}(\theta)^{0}}\right)_{*} \operatorname{IC}_{\zeta}^{\mathfrak{B}(\theta)^{0}}[-1]$. Otherwise, we have to show that $\mathcal{K}^{\theta}=0$.

By definition of IC, as $\mathcal{K}^{\theta}$ is a direct summand of $\mathfrak{s}^{\theta !}\left(\mathrm{IC}_{Z^{\theta}, \zeta}\right)$, it is placed in perverse degrees $\geq 1$. Restrict both sides of (35) to $\left.\operatorname{Mod}_{\tilde{M}}^{+, \theta}\right|_{\Delta_{X}}$ and apply the perverse cohomological truncation $\tau_{\geq 1}$. Using Lemma 5.2 and Proposition 5.1, we get

$$
\left.\operatorname{Loc}_{X, \zeta^{-1}}\left(U\left(\check{\mathfrak{u}}_{n}(P)\right)_{\theta}\right)[-1] \underset{\mathfrak{\rightarrow}(\theta),|\mathfrak{B}(\theta)| \neq 1}{\oplus} \operatorname{Loc}_{X, \zeta^{-1}}\left(\underset{\nu \in J}{\otimes} \operatorname{Sym}^{n_{\nu}}\left(\check{\mathfrak{u}}_{n}(P)_{\nu}\right)\right)[-1] \oplus \mathcal{K}^{\theta}\right|_{\widetilde{G}_{M, X}^{+, \theta}}
$$

As in ([2], Section 5.12), this implies the desired result. We used here that $U\left(\check{\mathfrak{u}}_{n}(P)\right)$ and $\operatorname{Sym}\left(\check{\mathfrak{u}}_{n}(P)\right)$ are non-canonically isomorphic as $\check{M}_{n}$-modules. Theorem 4.1 is proved.

\section{Composing Eisenstein SERIES}

6.0.1. In this section we prove Theorem 2.5, which is an analog of ([3], Theorem 2.3.10) in our setting.

Keep notations of Section 4.1. Let $B(M) \subset M$ be the Borel subgroup corresponding to the roots $\check{\alpha}_{i}, i \in \mathcal{J}_{M}$. As in ([3], Section 7.1) set $\widetilde{\operatorname{Bun}}_{B, P}=\widetilde{\operatorname{Bun}}_{P} \times_{\operatorname{Bun}_{M}} \overline{\operatorname{Bun}}_{B(M)}$. Set also $\widetilde{\operatorname{Bun}}_{\tilde{B}, \tilde{P}}=\widetilde{\operatorname{Bun}}_{\tilde{P}} \times_{\operatorname{Bun}_{M}} \overline{\operatorname{Bun}}_{\tilde{B}(M)}$. We have the cartesian square

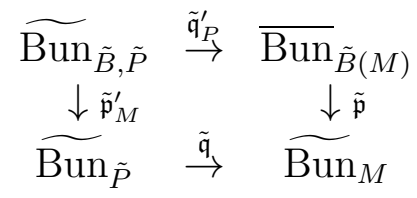

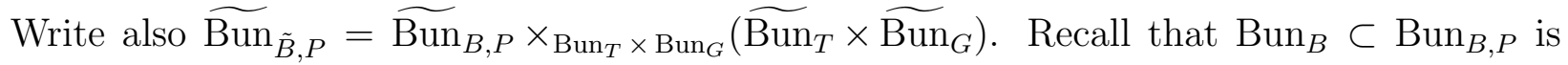
naturally an open substack. The preimage of $\operatorname{Bun}_{B}$ in $\widetilde{\operatorname{Bun}}_{\tilde{B}, P}$ identifies with the stack $\operatorname{Bun}_{\tilde{B}}$ from Section 3.1. Recall the perverse sheaf $\mathcal{L}_{\zeta} \otimes \operatorname{IC}\left(\operatorname{Bun}_{B, \tilde{G}}\right)$ viewed as a perverse sheaf on $\operatorname{Bun}_{\tilde{B}}$ via (21). Denote by $\mathrm{IC}_{B, P, \zeta}$ its intermediate extension under $\operatorname{Bun}_{\tilde{B}} \hookrightarrow \widehat{\operatorname{Bun}}_{\tilde{B}, P}$.

Proposition 6.1. The restriction of $\mathrm{IC}_{B, P, \zeta}$ under the projection $\widetilde{\operatorname{Bun}}_{\tilde{B}, \tilde{P}} \rightarrow \widetilde{\operatorname{Bun}}_{\tilde{B}, P}$ identifies canonically with

$$
\left(\tilde{\mathfrak{p}}_{M}^{\prime}\right)^{*} \mathrm{IC}_{\zeta} \otimes\left(\tilde{\mathfrak{q}}_{P}^{\prime}\right)^{*} \operatorname{IC}_{\zeta}\left[-\operatorname{dim} \operatorname{Bun}_{M}\right]
$$

Proof. Since $\operatorname{Bun}_{P} \rightarrow \operatorname{Bun}_{M}$ is smooth, $\operatorname{IC}_{\zeta}$ and $\left(j_{\tilde{P}}\right) ! j_{\tilde{P}}^{*} \mathrm{IC}_{\zeta}$ are ULA with respect to $\tilde{\mathfrak{q}}$ :

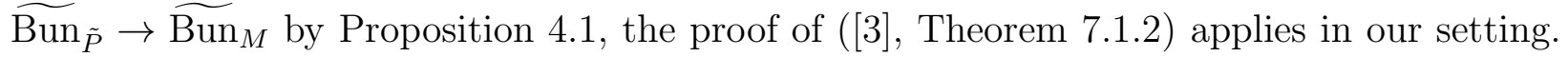
Using ([22], Proposition 4.8.5), one gets the desired isomorphism. 
Remark 6.1. There are two a priori different definitions of the ULA property. For a morphism $p_{1}: Y_{1} \rightarrow S_{1}$ and an object $L \in D\left(Y_{1}\right)$ the first definition of $L$ being ULA with respect to $p_{1}$ is ([7], Definition 2.12), and the second is ([3], Definition 5.1). In the latter one requires that the local acyclicity holds after any smooth base change $q: S \rightarrow S_{1}$, while in the former one requires it to hold after any base change $q: S \rightarrow S_{1}$. In the proof of Proposition 6.1 it was used that the two definitions are equivalent.

Recall the definition of the natural map $\mathfrak{r}_{P}: \widetilde{\operatorname{Bun}}_{B, P} \rightarrow \overline{\operatorname{Bun}}_{B}$ from([3], Proposition 7.1.5). A point of $\widetilde{\operatorname{Bun}}_{B, P}$ is a collection $\left(\mathcal{F}_{G}, \mathcal{F}_{M}, \kappa_{P}\right) \in \widetilde{\operatorname{Bun}}_{P},\left(\mathcal{F}_{M}, \mathcal{F}_{T}, \kappa_{M}\right) \in \overline{\operatorname{Bun}}_{B(M)}$. Here $\kappa_{M}$ is the collection of embeddings

$$
\kappa_{M}^{\check{\nu}}: \mathcal{L}_{\mathcal{F}_{T}}^{\check{\nu}} \hookrightarrow \mathcal{U}_{\mathcal{F}_{M}}^{\check{\nu}}, \quad \check{\nu} \in \Lambda_{M}^{+},
$$

and $\mathcal{U}^{\check{\nu}}$ denotes the corresponding Weyl module for $M$. Then $\mathfrak{r}_{P}$ sends this point to $\left(\mathcal{F}_{G}, \mathcal{F}_{T}, \kappa\right)$, where for $\check{\lambda} \in \check{\Lambda}^{+}$the map $\kappa^{\check{\lambda}}$ is the composition

$$
\mathcal{L}_{\mathcal{F}_{T}}^{\check{\lambda}} \stackrel{\kappa_{M}^{\check{\lambda}}}{\longrightarrow} U_{\mathcal{F}_{M}}^{\check{\check{\nu}}} \rightarrow\left(\mathcal{V}^{\check{\lambda}}\right)_{\mathcal{F}_{M}}^{U(P)} \stackrel{\kappa_{P}^{\check{\lambda}}}{\longrightarrow} V_{\mathcal{F}_{G}}^{\check{\lambda}}
$$

The map $\mathfrak{r}_{P}$ is representable and proper, it extends naturally to $\widetilde{\mathfrak{r}}_{P}: \widetilde{\operatorname{Bun}}_{\tilde{B}, P} \rightarrow \overline{\operatorname{Bun}}_{\tilde{B}}$. Recall that $\mathfrak{r}_{P}$ is an isomorphism over the open substack $\operatorname{Bun}_{B} \subset \overline{\operatorname{Bun}}_{B}$.

The following is an analog of ([3], Theorem 7.1.6) in our setting.

Theorem 6.1. One has canonically $\widetilde{\mathfrak{r}}_{P !} \mathrm{IC}_{B, P, \zeta} \widetilde{\rightarrow} \mathrm{IC}_{\zeta}$.

6.0.2. Proof of Theorem 6.1. Once our Theorem 4.1 is established, one easily adopts the proof of ([3], Theorem 7.1.6) to our setting. We indicate the corresponding notation and changes for the convenience of the reader.

Recall that $\Lambda_{M, G}^{+}=\Lambda_{M}^{+} \cap w_{0}^{M}\left(\Lambda_{G}^{\text {pos }}\right)$. Given a collection $\overline{\mu, \nu}$ consisting of $n_{1}, \ldots, n_{k} \in \mathbb{Z}_{>0}$ and pairwise different elements $\left(\mu_{1}, \nu_{1}\right), \ldots\left(\mu_{k}, \nu_{k}\right) \in\left(\Lambda_{M}^{\text {pos }} \times \Lambda_{M, G}^{+}\right)-0$ one sets $X^{\overline{\mu, \nu}}=X^{\left(n_{1}\right)} \times$ $\ldots \times X^{\left(n_{k}\right)}-\Delta$, where $\Delta$ is the divisor of all the diagonals. Write $D=\left(D_{1}, \ldots, D_{k}\right) \in X^{\overline{\mu, \nu}}$ for a point of this scheme.

The Hecke stack $\mathcal{H}_{M}^{\overline{\mu, \nu}}$ classifies $\left(D \in X^{\overline{\mu, \nu}}, \mathcal{F}_{M}^{\prime}, \mathcal{F}_{M} \in \operatorname{Bun}_{M}, \beta:\left.\mathcal{F}_{M}^{\prime} \widetilde{\rightarrow} \mathcal{F}_{M}\right|_{X-D}\right)$ such that $\mathcal{F}_{M}^{\prime}$ is in the position $\nu_{i}$ with respect to $\mathcal{F}_{M}$ at points of $D_{i}$.

By definition, $\overline{\mu, \nu} \operatorname{Bun}_{B, P}$ is the image of the locally closed embedding

$$
\operatorname{Bun}_{P} \times \operatorname{Bun}_{M} \mathcal{H}_{M}^{\overline{\mu, \nu}} \times{ }_{\operatorname{Bun}_{M}} \operatorname{Bun}_{B(M)} \hookrightarrow \widetilde{\operatorname{Bun}}_{B, P}
$$

The first stack classifies $\left(D \in X^{\overline{\mu, \nu}}, \mathcal{F}_{M}, \mathcal{F}_{M}^{\prime}, \beta\right) \in \mathcal{H}_{M}^{\overline{\mu, \nu}}, \mathcal{F}_{P} \in$ Bun $_{P}$ with an isomorphism $\mathcal{F}_{P} \times_{P} M \underset{\rightarrow}{\rightarrow} \mathcal{F}_{M}$, a $B(M)$-torsor $\mathcal{F}_{B(M)}$ with $\mathcal{F}_{T}:=\mathcal{F}_{B(M)} \times_{B(M)} T$, an isomorphism $\mathcal{F}_{B(M)} \times_{B(M)}$ $M \widetilde{\rightarrow} \mathcal{F}_{M}^{\prime}$. Its image in $\widetilde{\operatorname{Bun}}_{B, P}$ is the collection $\left(\mathcal{F}_{G}, \mathcal{F}_{M}^{\prime}, \kappa\right) \in \widetilde{\operatorname{Bun}}_{P},\left(\mathcal{F}_{M}^{\prime}, \mathcal{F}_{T}^{\prime}, \kappa_{M}\right) \in \overline{\operatorname{Bun}}_{B(M)}$, where $\mathcal{F}_{T}^{\prime}=\mathcal{F}_{T}\left(-\sum_{i} \mu_{i} D_{i}\right)$ and $\mathcal{F}_{G}=\mathcal{F}_{P} \times_{P} G$.

For $\lambda=\sum_{i} m_{i} \alpha_{i} \in \Lambda_{G}^{\text {pos }}$ the locally closed substack $\overline{\operatorname{Bun}}_{B}^{\lambda} \subset \overline{\operatorname{Bun}}_{B}$ is defined as the image of the locally closed immersion

$$
\prod_{i \in \mathcal{J}} X^{\left(m_{i}\right)} \times \operatorname{Bun}_{B} \rightarrow \overline{\operatorname{Bun}}_{B}
$$


sending $\left(\left(D_{i}\right)_{i \in \mathcal{J}}, \mathcal{F}_{B}\right)$ to $\left(\mathcal{F}_{T}^{\prime}, \mathcal{F}_{G}, \kappa\right)$, where $\mathcal{F}_{T}^{\prime}=\mathcal{F}_{T}\left(-\sum_{i} \alpha_{i} D_{i}\right)$ for $\mathcal{F}_{T}=\mathcal{F}_{B} \times_{B} T, \mathcal{F}_{G}=$ $\mathcal{F}_{B} \times_{B} G$.

For $\overline{\mu, \nu}$ as above, $\lambda \in \Lambda_{G}^{\text {pos }}$ one sets

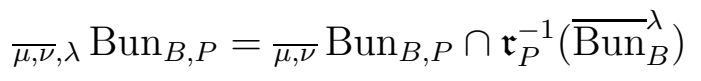

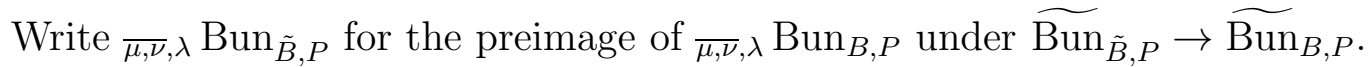

We set $|\overline{\mu, \nu}|=\sum_{i=1}^{k} n_{i}\left(\nu_{i}-\mu_{i}\right) \in \Lambda$. Theorem 6.1 is reduced to the following.

Proposition 6.2. For $\overline{\mu, \nu}, \lambda \in \Lambda_{G}^{\text {pos }}$ as above the following holds:

(i) The *-restriction of $\mathrm{IC}_{B, P, \zeta}$ to $\overline{\mu, \nu, \lambda} \operatorname{Bun}_{\tilde{B}, P}$ lives in perverse degrees $\leq-\left\langle\lambda+|\overline{\mu, \nu}|, \check{\rho}_{M}\right\rangle$, and the inequality is strict unless $\lambda=0$.

(ii) The fibres of $\mathfrak{r}_{P}: \overline{\mu, \nu, \lambda} \operatorname{Bun}_{\tilde{B}, P} \rightarrow \overline{\operatorname{Bun}}_{B}^{\lambda}$ are of dimension $\leq\left\langle\lambda+|\overline{\mu, \nu}|, \check{\rho}_{M}\right\rangle$.

Proof. The proof of ([3], Proposition 7.1.8) applies. The only change is that one uses Theorem 4.1 (and Proposition 6.1) to garantee that the $*$-restriction of $\mathrm{IC}_{B, P, \zeta}$ to $\frac{\mu, \nu}{\text { Bun }} \tilde{B, P}$ has smooth cohomology sheaves.

Proof of Theorem 2.5. For $K \in \mathrm{D}_{\zeta}\left(\widetilde{\operatorname{Bun}}_{T}\right)$ one has

$$
\operatorname{Eis}_{M}^{G} \operatorname{Eis}_{T}^{M}(K) \widetilde{\rightarrow} \tilde{\mathfrak{p}}_{!}\left(\mathrm{IC}_{\zeta} \otimes \tilde{\mathfrak{q}}^{*}\left(\tilde{\mathfrak{p}}_{M}\right) !\left(\tilde{\mathfrak{q}}_{M}^{*} K \otimes \mathrm{IC}_{\zeta}\right)\right)\left[-\operatorname{dim} \operatorname{Bun}_{T}-\operatorname{dim} \operatorname{Bun}_{M}\right]
$$

Using the projection formula and Proposition 6.1, this identifies with

$$
\tilde{\mathfrak{p}}_{!}\left(\tilde{\mathfrak{p}}_{M}^{\prime}\right) !\left(\mathrm{IC}_{B, P, \zeta} \otimes\left(\tilde{\mathfrak{q}}_{P}^{\prime}\right)^{*} \tilde{\mathfrak{q}}_{M}^{*}\right)\left[-\operatorname{dim} \operatorname{Bun}_{T}\right]
$$

The composition $\tilde{\mathfrak{q}}_{P}^{\prime} \tilde{\mathfrak{q}}_{M}$ coincides with $\widetilde{\operatorname{Bun}}_{\tilde{B}, \tilde{P}} \rightarrow \widetilde{\operatorname{Bun}}_{\tilde{B}, P} \stackrel{\mathfrak{\mathfrak { x }}_{P}}{\rightarrow} \overline{\operatorname{Bun}}_{\tilde{B}} \stackrel{\tilde{\mathfrak{q}}}{\rightarrow} \widetilde{\operatorname{Bun}}_{T}$, and the composition $\tilde{\mathfrak{p}}_{\mathfrak{\mathfrak { p }}}^{\prime}{ }_{M}$ coincides with $\widetilde{\operatorname{Bun}}_{\tilde{B}, \tilde{P}} \rightarrow \widetilde{\operatorname{Bun}}_{\tilde{B}, P} \stackrel{\mathfrak{\mathfrak { r }}_{P}}{\rightarrow} \overline{\operatorname{Bun}}_{\tilde{B}} \stackrel{\tilde{\mathfrak{p}}}{\rightarrow} \widetilde{\operatorname{Bun}}_{G}$. So, (36) identifies with

$$
\tilde{\mathfrak{p}}_{!}\left(\left(\tilde{\mathfrak{r}}_{P}\right) ! \mathrm{IC}_{B, P, \zeta} \otimes \tilde{\mathfrak{q}}^{*} K\right)\left[-\operatorname{dim} \operatorname{Bun}_{T}\right] \widetilde{\rightarrow} \operatorname{Eis}_{T}^{G}(K)
$$

by Theorem 6.1.

\section{THE CASE OF $G=\mathrm{SL}_{2}$}

7.1. Precisions. In this section we get some more precise results for $G=\mathrm{SL}_{2}$. Keep notations of Section 2, Let $e=n$ for $n$ odd (resp., $e=\frac{n}{2}$ for $n$ even). Then $\Lambda^{\sharp}=e \Lambda$. The unique simple coroot of $G$ is denoted $\alpha$, the simple root of $\check{G}_{n}$ is $n \alpha$. For $n$ even one gets $\check{G}_{n} \widetilde{\rightarrow} \mathrm{SL}_{2}$, and $\check{G}_{n} \widetilde{\rightarrow} \mathrm{PSL}_{2}$ for $n$ odd. Recall that $\check{h}=2$

Let $\mathcal{L}_{c}$ be the line bundle on $\operatorname{Bun}_{G}$ with fibre $\operatorname{det} \mathrm{R} \Gamma\left(X, \mathcal{O}^{2}\right) \otimes \operatorname{det} \mathrm{R} \Gamma(X, M)^{-1}$ at $M \in$ $\operatorname{Bun}_{G}$. The restriction of $\mathcal{L}_{c}$ to $\operatorname{Bun}_{T}$ is also denoted $\mathcal{L}_{c}$.

Identify $T$ with $\mathbb{G}_{m}$ via the coroot $\alpha: \mathbb{G}_{m} \widetilde{\rightarrow} T$, so Bun $_{1} \widetilde{\rightarrow}$ Bun $_{T}$. The isomorphism $\mathbb{Z} \widetilde{\rightarrow} \Lambda^{\sharp}, 1 \mapsto e$ yields $\mathbb{G}_{m} \widetilde{\rightarrow} T^{\sharp}$, so that $i_{X}: \operatorname{Bun}_{1}=\operatorname{Bun}_{T^{\sharp}} \rightarrow \operatorname{Bun}_{T}=\operatorname{Bun}_{1}$ sends $\mathcal{E}$ to $\mathcal{E}^{e}$. The line bundle $\tau$ on $\operatorname{Bun}_{T^{\sharp}}$ is chosen as in ([17], 5.2.6, Example (1)). Namely, if $n$ is odd then

$$
\tau_{\mathcal{E}}=\operatorname{det} \mathrm{R} \Gamma(X, \mathcal{O})^{2 n} \otimes \operatorname{det} \mathrm{R} \Gamma(X, \mathcal{E})^{-n} \otimes \operatorname{det} \mathrm{R} \Gamma\left(X, \mathcal{E}^{-1}\right)^{-n}
$$


for $\mathcal{E} \in \operatorname{Bun}_{1}=\operatorname{Bun}_{T^{\sharp}}$. If $n$ is even then we first pick a super line bundle $\mathcal{L}_{1}$ on $\operatorname{Bun}_{1}$ equipped with $\mathcal{L}_{1}^{2} \underset{\rightarrow}{\rightarrow} \mathcal{L}_{c}$ on $\operatorname{Bun}_{T}$. Then $\tau=\mathcal{L}_{1}^{e}$ on $\operatorname{Bun}_{1}$.

Recall the action of $Z(G)=\mu_{2}$ on $\widetilde{\operatorname{Bun}}_{G}$ by 2-automorphisms (see Section 3.5). Denote by $\mathrm{D}_{\zeta,+}\left(\widetilde{\operatorname{Bun}}_{G}\right)$ and by $\mathrm{D}_{\zeta,-}\left(\widetilde{\operatorname{Bun}}_{G}\right)$ the full subcategory of $\mathrm{D}_{\zeta}\left(\widetilde{\operatorname{Bun}_{G}}\right)$, where $-1 \in \mu_{2}$ acts as 1 and -1 respectively. As in [18, we get

$$
\mathrm{D}_{\zeta}\left(\widetilde{\operatorname{Bun}}_{G}\right) \widetilde{\rightarrow} \mathrm{D}_{\zeta,+}\left(\widetilde{\operatorname{Bun}_{G}}\right) \times \mathrm{D}_{\zeta,-}\left(\widetilde{\operatorname{Bun}_{G}}\right)
$$

For $n$ even the category $\operatorname{Rep}\left(\check{G}_{n}\right)$ is $\mathbb{Z} / 2 \mathbb{Z}$-graded according to the action of the center of

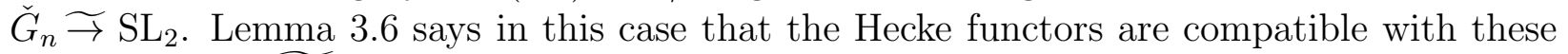
gradings of $\mathrm{D}_{\zeta}\left(\widetilde{\operatorname{Bun}}_{G}\right)$ and $\operatorname{Rep}\left(\check{G}_{n}\right)$.

7.1.1. Take $P=B$. Let us reformulate Corollary 5.1 more explicitly in this case. The stack Bun $_{B}$ classifies exact sequences

$$
0 \rightarrow \mathcal{E} \rightarrow M \rightarrow \mathcal{E}^{-1} \rightarrow 0
$$

on $X$ with $\mathcal{E} \in \operatorname{Bun}_{1}$. The stack $\overline{\operatorname{Bun}}_{B}$ classifies $M \in \operatorname{Bun}_{G}$ and a subsheaf $\mathcal{E} \hookrightarrow M$, where $\mathcal{E} \in \operatorname{Bun}_{1}$. The line bundle $\mathcal{L}_{T}$ on $\operatorname{Bun}_{T}=\operatorname{Bun}_{1}$ is such that its fibre at $\mathcal{E}$ is

$$
\frac{\operatorname{det} \mathrm{R} \Gamma(X, \mathcal{O})^{2}}{\operatorname{det} \mathrm{R} \Gamma\left(X, \mathcal{E}^{2}\right) \otimes \operatorname{det} \mathrm{R} \Gamma\left(X, \mathcal{E}^{-2}\right)}=\frac{\operatorname{det} \mathrm{R} \Gamma(X, \mathcal{O})^{8}}{\operatorname{det} \mathrm{R} \Gamma(X, \mathcal{E})^{4} \otimes \operatorname{det} \mathrm{R} \Gamma\left(X, \mathcal{E}^{-1}\right)^{4}}
$$

There is an isomorphism $\mathcal{L}_{c}^{4} \widetilde{\rightrightarrows} \mathcal{L}$ over $\operatorname{Bun}_{G}$, whose restriction to $\mathrm{Bun}_{T}$ is compatible with the isomorphism (39).

The stack $\widetilde{\operatorname{Bun}}_{T}$ is the gerb of $4 n$-th roots of $\mathcal{L}_{T}$ over $\operatorname{Bun}_{T}$. The map $\bar{c}_{P}: \Lambda_{G, B}^{\text {pos,pos }} \rightarrow \Lambda_{G, B}^{\sharp, \text { pos }}$ is injective, its image equals $n \alpha \mathbb{Z}_{+}$.

For $\theta=m \alpha \in \Lambda_{G, B}^{\text {pos }}$ the stack ${ }_{\theta} \overline{\operatorname{Bun}}_{B}$ classifies $D \in X^{(m)}$ and an exact sequence on $X$

$$
0 \rightarrow \mathcal{E}(D) \rightarrow M \rightarrow \mathcal{E}^{-1}(-D) \rightarrow 0
$$

with $\mathcal{E} \in \operatorname{Bun}_{1}$. The $*$-restriction of $\mathrm{IC}_{\zeta}$ to ${ }_{\theta} \overline{\mathrm{Bun}}_{\tilde{B}}$ vanishes unless $m \in n \mathbb{Z}$, in the latter case $\theta$ admits a unique $\mathfrak{B}(\theta)=\frac{m}{n} \nu$, where $\nu=n \alpha \in J$, and the map $X^{\mathfrak{B}(\theta)} \rightarrow X^{\theta}$ becomes $X^{\left(\frac{m}{n}\right)} \rightarrow X^{(m)}, D \mapsto n D$. By Corollary 5.1,

$$
\left.\mathrm{IC}_{\zeta}\right|_{\theta} \overline{\operatorname{Bun}}_{\tilde{B}} \widetilde{\rightrightarrows} \nu_{B}^{*} \mathrm{IC}_{\mathrm{Bun}_{M}, \zeta}^{\mathfrak{B}(\theta)}\left[\operatorname{dim} \cdot \operatorname{rel}\left(\nu_{B}\right)+\frac{m}{n}\right]
$$

where $\nu_{B}: \operatorname{Bun}_{B} \times{ }_{\mathrm{Bun}_{T}} \widetilde{\operatorname{Mod}_{\mathrm{Bun}_{T}}} \rightarrow \widetilde{\operatorname{Mod}_{\text {Bun }}}+\frac{+, \theta}{+, \theta}$ is the projection. The perverse sheaf $\mathrm{IC}_{\mathrm{Bun}_{M}, \zeta}^{\mathfrak{B}(\theta)}$ is described as follows.

Lemma 7.1. Let $\mathcal{E} \in \mathrm{Bun}_{1}$, let $D$ be an effective divisor on $X$. Then there is a canonical $\mathbb{Z} / 2 \mathbb{Z}$-graded isomorphism

$$
\frac{\operatorname{det} \mathrm{R} \Gamma(X, \mathcal{E}) \otimes \operatorname{det} \mathrm{R} \Gamma\left(X, \mathcal{E}^{-1}\right)}{\operatorname{det} \mathrm{R} \Gamma\left(X, \mathcal{E}(n D) \otimes \operatorname{det} \mathrm{R} \Gamma\left(X, \mathcal{E}^{-1}(-n D)\right)\right.} \underset{\rightarrow}{\rightarrow}\left(\frac{\operatorname{det} \mathrm{R} \Gamma\left(X, \mathcal{O}_{D}\right)}{\operatorname{det} \mathrm{R} \Gamma\left(X,\left.\mathcal{E}^{2}(n D)\right|_{D}\right)}\right)^{n}
$$


Proof One has canonically $\operatorname{det} \mathrm{R} \Gamma(X, \mathcal{E}(n D) / \mathcal{E}) \stackrel{\rightarrow}{\rightarrow} \otimes_{r=1}^{n} \operatorname{det} \mathrm{R} \Gamma\left(X,\left.\mathcal{E}(r D)\right|_{D}\right)$ and

$$
\operatorname{det} \mathrm{R} \Gamma\left(X, \mathcal{E}^{-1} / \mathcal{E}^{-1}(-n D)\right) \stackrel{\Im}{\rightarrow} \otimes_{r=1}^{n} \operatorname{det} \mathrm{R} \Gamma\left(X,\left.\mathcal{E}^{-1}((r-n) D)\right|_{D}\right)
$$

Using 7.1 .2 below, we get for $1 \leq r \leq n$

$$
\frac{\operatorname{det} \operatorname{R} \Gamma\left(X,\left.\mathcal{E}^{-1}((r-n) D)\right|_{D}\right)}{\operatorname{det} \operatorname{R} \Gamma\left(X,\left.\mathcal{E}(r D)\right|_{D}\right)} \Im \frac{\operatorname{det} \mathrm{R} \Gamma\left(X, \mathcal{O}_{D}\right)}{\operatorname{det} \operatorname{R} \Gamma\left(X,\left.\mathcal{E}^{2}(n D)\right|_{D}\right)}
$$

Our claim follows.

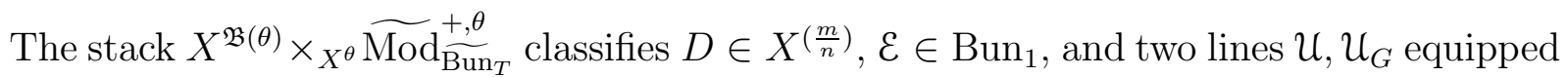
with isomorphisms $\mathcal{U}_{G}^{N} \widetilde{\rightrightarrows}\left(\mathcal{L}_{T}\right)_{\mathcal{E}(n D)}, \mathcal{U}^{N} \widetilde{\Im}\left(\mathcal{L}_{T}\right)_{\mathcal{E}}$. The stack ${ }_{\theta} \overline{\mathrm{Bun}}_{\tilde{B}} \times_{X^{\theta}} X^{\mathfrak{B}(\theta)}$ classifies the same data together with an exact sequence

$$
0 \rightarrow \mathcal{E}(n D) \rightarrow M \rightarrow \mathcal{E}^{-1}(-n D) \rightarrow 0,
$$

Recall that $N=4 n$. One has an isomorphism

$$
X^{\mathfrak{B}(\theta)} \times_{X^{\theta}} \operatorname{Mod}_{\mathrm{Bun}_{T}}^{+, \theta} \times B\left(\mu_{N}\right) \widetilde{\rightrightarrows} X^{\mathfrak{B}(\theta)} \times_{X^{\theta}} \widetilde{\operatorname{Mod}_{\mathrm{Bun}_{T}}^{+, \theta}}
$$

sending $\left(D, \mathcal{E}, \mathcal{U}_{G}, \mathcal{U}_{G}^{N} \widetilde{\rightrightarrows}\left(\mathcal{L}_{T}\right)_{\mathcal{E}(n D)}, \mathcal{U}_{0}^{N} \widetilde{\Im} k\right)$ to $\left(D, \mathcal{E}, \mathcal{U}_{G}, \mathcal{U}\right)$, where

$$
\mathcal{U}=\mathcal{U}_{G} \otimes \mathcal{U}_{0}^{-1} \otimes \frac{\operatorname{det} \mathrm{R} \Gamma\left(X,\left.\mathcal{E}^{2}(n D)\right|_{D}\right)}{\operatorname{det} \mathrm{R} \Gamma\left(X, \mathcal{O}_{D}\right)}
$$

is equipped with the isomorphism $\mathcal{U}^{N} \widetilde{\neg}\left(\mathcal{L}_{T}\right)_{\mathcal{E}}$ given by Lemma 7.1. The perverse sheaf $\mathrm{IC}_{\mathrm{Bun}_{M}, \zeta}^{\mathfrak{B}(\theta)}$ via (40) identifies non-canonically with

$$
\mathrm{IC}\left(X^{\mathfrak{B}(\theta)} \times_{X^{\theta}} \operatorname{Mod}_{\overparen{\mathrm{Bun}_{T}}}^{+, \theta}\right) \otimes \mathcal{L}_{\zeta}
$$

7.1.2. We need the following. Let $D$ be an effective divisor on $X, \mathcal{A}, \mathcal{B} \in \operatorname{Bun}_{1}$. There is a canonical $\mathbb{Z} / 2 \mathbb{Z}$-graded isomorphism

$$
\frac{\operatorname{det} \mathrm{R} \Gamma\left(X,\left.\mathcal{A}\right|_{D}\right)}{\operatorname{det} \mathrm{R} \Gamma\left(X,\left.\mathcal{B}\right|_{D}\right)} \underset{\rightarrow}{\rightarrow} \frac{\operatorname{det} \mathrm{R} \Gamma\left(X,\left.\mathcal{A} \otimes \mathcal{B}^{-1}\right|_{D}\right)}{\operatorname{det} \mathrm{R} \Gamma\left(X, \mathcal{O}_{D}\right)}
$$

7.1.3. Let $\theta=m \alpha \in \Lambda_{G, B}^{\text {pos }}$. For $\mathcal{E} \in \operatorname{Bun}_{1}=\operatorname{Bun}_{T}$ write $Z_{\mathcal{E}}^{\theta}$ for the Zastava space with the 'background' $T$-torsor $\mathcal{E}^{-1}$. Then $Z_{\varepsilon}^{\theta}$ is a vector bundle over $X^{\theta}$ whose fibre at $D \in X^{(m)}$ is $\mathcal{E}^{2}(D) / \mathcal{E}^{2} \Im \operatorname{Ext}^{1}\left(\mathcal{E}^{-1} / \mathcal{E}^{-1}(-D), \mathcal{E}\right)$. It is understood that a point of $Z_{\mathcal{E}}^{\theta}$ gives rise to a diagram on $X$

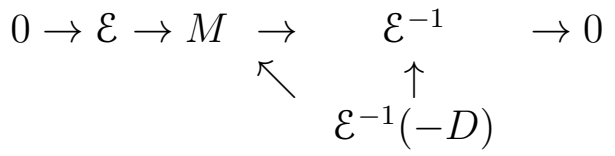

The group scheme $M_{X^{\theta}}$ acts trivially on $\operatorname{Mod}_{M}^{+, \theta}=X^{\theta}$. If $D \in X^{(m)}$ is given by $D=$ $\sum n_{k} x_{k}$ with $x_{k}$ pairwise different then the fibre of $M_{X^{\theta}}$ at $D$ is $\prod_{k} \mathcal{O}_{x_{k}}^{*}$. The action of $M_{X^{\theta}}$ on $Z^{\theta}$ from Section 5.1 .1 becomes as follows. The element $g=\left(g_{k}\right) \in \prod_{k} \mathcal{O}_{x_{k}}^{*}$ acts on $v=\left(v_{k}\right) \in \prod_{k} \mathcal{E}^{2}\left(n_{k} x_{k}\right) / \mathcal{E}^{2}$ as $g^{2} v=\left(g_{k}^{2} v_{k}\right)$. 
Let $\mathbb{G}^{\theta}$ denote the group scheme over $X^{\theta}$ whose fibre at $D$ is $(\mathcal{O} / \mathcal{O}(-D))^{*}$. The action of $M_{X^{\theta}}$ on $Z^{\theta}$ factors through an action of $\mathbb{G}^{\theta}$.

Write $\overline{\mathcal{L}}$ for the line bundle over $X^{\theta}$, whose fibre at $D$ is

$$
\frac{\left(\mathcal{L}_{c}\right)_{\mathcal{E}(D)}}{\left(\mathcal{L}_{c}\right)_{\mathcal{E}}}=\frac{\operatorname{det} \mathrm{R} \Gamma(X, \mathcal{E}) \otimes \operatorname{det} \mathrm{R} \Gamma\left(X, \mathcal{E}^{-1}\right)}{\operatorname{det} \mathrm{R} \Gamma(X, \mathcal{E}(D)) \otimes \operatorname{det} \mathrm{R} \Gamma\left(X, \mathcal{E}^{-1}(-D)\right)} \underset{\rightarrow}{\rightarrow} \frac{\operatorname{det} \mathrm{R} \Gamma\left(X, \mathcal{O}_{D}\right)}{\operatorname{det} \mathrm{R} \Gamma\left(X,\left.\mathcal{E}^{2}(D)\right|_{D}\right)}
$$

Here the second isomorphism is given by Lemma 7.1. The restriction of $\overline{\mathcal{L}}$ to $Z_{\mathcal{E}}^{\theta}$ is also denoted $\overline{\mathcal{L}}$. Then $\tilde{Z}_{\mathcal{E}}^{\theta}$ is the gerb of $4 n$-th roots of $\overline{\mathcal{L}}^{4}$. Write also $\tilde{Z}_{\mathcal{E}, c}^{\theta}$ for the gerb of $n$-th roots of $\overline{\mathcal{L}}$. We have a natural map $\tilde{Z}_{\mathcal{\varepsilon}, c}^{\theta} \rightarrow \tilde{Z}_{\mathcal{E}}^{\theta}$ making $\tilde{Z}_{\mathcal{\varepsilon}}^{\theta}$ a trivial $\mu_{4}$-gerb over $\tilde{Z}_{\mathcal{\varepsilon}, c}^{\theta}$. Let $\mathrm{IC}_{Z_{c}^{\theta}, \bar{\zeta}}$ denote the restriction of $\mathrm{IC}_{Z^{\theta}, \zeta}$ to $\tilde{Z}_{\mathcal{E}, c}^{\theta}$.

For a point $\left(D, v \in \mathcal{E}^{2}(D) / \mathcal{E}^{2}, \mathcal{U}\right) \in \tilde{Z}_{\mathcal{E}, c}^{\theta}$ with $\mathcal{U}^{n} \widetilde{\Im} \overline{\mathcal{L}}_{D}$ note that $a \in \mu_{n}(k) \subset$ Aut $\mathcal{U}$ acts on $\mathrm{IC}_{Z_{c}^{\theta}, \bar{\zeta}}$ as $\bar{\zeta}(a)^{-1}$.

The open subscheme $Z_{\max }^{\theta} \subset Z_{\varepsilon}^{\theta}$ classifies $\left(D, \sigma \in \mathcal{E}^{2}(D) / \mathcal{E}^{2}\right)$ such that for any $0 \leq$ $D^{\prime}<D$ we have $\sigma \notin \mathcal{E}^{2}\left(D^{\prime}\right) / \mathcal{E}^{2}$. Over $Z_{\text {max }}^{\theta}$ we have a canonical section of $\overline{\mathcal{L}}$ given by the isomorphisms

$\operatorname{det} \mathrm{R} \Gamma(X, \mathcal{E}) \otimes \operatorname{det} \mathrm{R} \Gamma\left(X, \mathcal{E}^{-1}\right) \widetilde{\rightarrow} \operatorname{det} \mathrm{R} \Gamma(X, M) \widetilde{\rightarrow} \operatorname{det} \mathrm{R} \Gamma(X, \mathcal{E}(D)) \otimes \operatorname{det} \mathrm{R} \Gamma\left(X, \mathcal{E}^{-1}(-D)\right)$

Let $\check{Z}_{\mathcal{E}}^{\theta} \rightarrow X^{\theta}$ denote the dual vector bundle, so its fibre over $D$ is $\mathcal{E}^{-2} \otimes \Omega / \mathcal{E}^{-2} \otimes \Omega(-D)$. Denote by $\tilde{\tilde{Z}}_{\varepsilon, c}^{\theta}$ the gerb of $n$-th roots of $\overline{\mathcal{L}}$ over $\check{Z}_{\mathcal{\varepsilon}}^{\theta}$.

7.2. Fourier coefficients. The purpose of this section is to establish some results about the Fourier transform of $\mathrm{IC}_{Z_{c}^{\theta}, \bar{\zeta}}$ over $\tilde{\check{Z}}_{\mathcal{\varepsilon}, c}^{\theta}$. This is important in view of a relation with the theory of Weyl group multiple Dirichlet series (see [4], [6] for a survey).

7.2.1. We need the following observation. Let $X$ denote the stack classifying a 1-dimensional $k$-vector spaces $L, U$ together with $U^{n} \widetilde{\rightarrow} L$, and $v \in L$. This is a vector bundle over the stack $B\left(\mathbb{G}_{m}\right)$ classifying a line $U$. Let $\check{X}$ denote the dual vector bundle over $B\left(\mathbb{G}_{m}\right)$, this is the stack classifying $U, L, U^{n} \widetilde{\rightarrow} L$ and $v^{*} \in L^{*}$. Let $X^{0} \subset X$ be the open substack given by $v \neq 0$. We have an isomorphism $\tau_{X}: X^{0} \widetilde{\Im} B\left(\mu_{n}\right)$ sending the above point to $U$ equipped with the trivialization $U^{n} \widetilde{\rightrightarrows} k$ obtained from $k \widetilde{\rightarrow} L, 1 \mapsto v$. For the natural map $a:$ Spec $k \rightarrow B\left(\mu_{n}\right)$ let $\mathcal{L}_{\bar{\zeta}}$ denote the direct summand in $a_{*} \overline{\mathbb{Q}}_{\ell}$ on which $\mu_{n}(k)$ acts by $\bar{\zeta}$. Let $\mathcal{L}_{\bar{\zeta}, \text { ex }}$ denote the intermediate extension of $\tau_{x}^{*} \mathcal{L}_{\bar{\zeta}}$ under $X^{0} \hookrightarrow X$.

Denote by $\check{X}^{0} \subset \check{X}$ the open substack given by $v^{*} \neq 0$. Let $\tau_{\check{x}}: \check{X}^{0} \widetilde{\rightarrow} B\left(\mu_{n}\right)$ be the isomorphism sending the above point to $U$ equipped with the composition $U^{n} \stackrel{\rightrightarrows}{\rightarrow} \stackrel{v^{*}}{\rightarrow} k$. Write $\mathcal{L}_{\bar{\zeta}, \check{e} x}$ for the intermediate extension of $\tau_{\check{x}}^{*} \mathcal{L}_{\bar{\zeta}}$ to $\check{X}$. For $n \geq 2$ there is a 1-dimensional $\overline{\mathbb{Q}}_{\ell}$-vector space $\mathcal{C}_{0}$ and a canonical isomorphism

$$
\operatorname{Four}_{\psi}\left(\mathcal{L}_{\bar{\zeta}^{-1}, e x}\right) \widetilde{\rightrightarrows} \mathcal{C}_{0} \otimes \mathcal{L}_{\bar{\zeta}^{-1}, e x}
$$


7.2.2. Example $\theta=\alpha$. In this case $Z_{\mathcal{\varepsilon}}^{\theta}$ is the total space of the line bundle $\mathcal{E}^{2} \otimes \Omega^{-1}$ over $X$. The line bundle $\overline{\mathcal{L}}$ over $X$ identifies with $\mathcal{E}^{-2} \otimes \Omega$. We have a map $p_{\check{X}}: \tilde{Z}_{\mathcal{\varepsilon}, c}^{\theta} \rightarrow \check{X}$ given by $L=\mathcal{E}^{-2} \otimes \Omega$. Then

$$
p_{\check{x}}^{*} \mathcal{L}_{\bar{\zeta}^{-1}, \check{x}}[2] \stackrel{\rightarrow}{\rightarrow} \mathrm{IC}_{Z_{c}^{\theta}, \bar{\zeta}}
$$

canonically. We also have the natural map $p_{x}: \check{\tilde{Z}}_{\varepsilon, c}^{\theta} \rightarrow X$ defined by the same formula. Set $\mathrm{IC}_{\check{Z}_{c}^{\theta}, \bar{\zeta}}=p_{X}^{*} \mathcal{L}_{\bar{\zeta}^{-1}, e x}[2]$, this is an irreducible perverse sheaf. Now from (43) we get an isomorphism

$$
\operatorname{Four}_{\psi}\left(\mathrm{IC}_{Z_{c}^{\theta}, \bar{\zeta}}\right) \widetilde{\rightarrow} \mathrm{C}_{0} \otimes \mathrm{IC}_{\check{Z}_{c}^{\theta}, \bar{\zeta}}
$$

7.2.3. Generalization. It is natural to consider the following generalization of $\mathrm{IC}_{Z_{c}^{\theta} \bar{\zeta} \bar{\zeta}}$. Let $L$ be a line bundle on $X$. Let $\theta=m \alpha, m \geq 0$, so $X^{(m)} \widetilde{\Im} X^{\theta}$ via the map $D \mapsto D \alpha$. Let $X^{\theta, r s s} \subset X^{\theta}$ be the open subscheme of reduced divisors. Write Sign for the sign local system on $X^{\theta, r s s}$. Let ${ }_{L} Z^{\theta}$ be the vector bundle over $X^{\theta}$ with fibre $L(D) / L$ at $D \in X^{(m)}$. Let $\overline{\mathcal{L}}$ be the line bundle over $X^{\theta}$ with fibre

$$
\frac{\operatorname{det} \operatorname{R} \Gamma\left(X, \mathcal{O}_{D}\right)}{\operatorname{det} \operatorname{R} \Gamma(X, L(D) / L)}
$$

at $D$. Let ${ }_{L} \tilde{Z}^{\theta}$ be the gerb of $n$-th roots of $\overline{\mathcal{L}}$ over ${ }_{L} Z^{\theta}$. Write ${ }_{L} Z_{\text {max }}^{\theta} \subset{ }_{L} Z^{\theta}$ for the open subscheme given by $v \in L(D) / L$ such that for any $0 \leq D^{\prime}<D, v \notin L\left(D^{\prime}\right) / L$. Let

$$
{ }_{L} Z_{\max }^{\theta, r s s} \subset{ }_{L} Z_{\max }^{\theta}
$$

be the open subscheme given by the property that $D$ is multiplicity free.

If $D=\sum_{i} x_{i} \in X^{\theta}$ with $x_{i}$ pairwise different then the fibre of $\overline{\mathcal{L}}$ at $D$ is $\otimes_{i}\left(L^{-1} \otimes \Omega\right)_{x_{i}}$, where each $\left(L^{-1} \otimes \Omega\right)_{x_{i}}$ is of parity zero, so the order does not matter. Besides, $L(D) / L=$ $\oplus_{i} L\left(x_{i}\right) / L$. So, a point $v \in{ }_{L} Z_{\text {max }}^{\theta, r s s}$ is a collection $0 \neq v_{i} \in L\left(x_{i}\right) / L$ for all $i$. This gives a trivialization of each line $L\left(x_{i}\right) / L$, hence also a trivialization of $\overline{\mathcal{L}}_{D}$ as the tensor product thereof. So, we get a trivialization of $\overline{\mathcal{L}}$ over ${ }_{L} Z_{\text {max }}^{\theta, r s s}$.

We denote by ${ }_{L} \tilde{Z}_{\max }^{\theta, r s s}$ the restriction of the gerb ${ }_{L} \tilde{Z}^{\theta}$ to this open subscheme. The above trivialization yields an isomorphism

$$
{ }_{L} Z_{\max }^{\theta, r s s} \times B\left(\mu_{n}\right) \widetilde{\Im}_{L} \tilde{Z}_{\max }^{\theta, r s s}
$$

Consider IC $\otimes \mathcal{L}_{\bar{\zeta}^{-1}}$ as a perverse sheaf on ${ }_{L} \tilde{Z}_{\text {max }}^{\theta, r s s}$ via (45). Its intermediate extension to ${ }_{L} \tilde{Z}^{\theta}$ is denoted $\mathrm{IC}_{L} Z^{\theta}, \bar{\zeta}$. For a point

$$
(D, v \in L(D) / L, \mathcal{U}) \in{ }_{L} \tilde{Z}^{\theta}
$$

with $\mathcal{U}^{n} \widetilde{\Im} \overline{\mathcal{L}}_{D}$ the element $a \in \mu_{n}(k) \subset \operatorname{Aut}(\mathcal{U})$ acts on $\mathrm{IC}_{L} Z^{\theta}, \bar{\zeta}$ as $\bar{\zeta}^{-1}(a)$.

The dual vector bundle ${ }_{L} \check{Z}^{\theta} \rightarrow X^{\theta}$ has the fibre $L^{-1} \otimes \Omega / L^{-1} \otimes \Omega(-D)$ at $D$. Let ${ }_{L} \check{\tilde{Z}}^{\theta}$ be the gerb of $n$-th roots of $\overline{\mathcal{L}}$ over ${ }_{L} \check{Z}^{\theta}$. We define ${ }_{L} \check{Z}_{\text {max }}^{\theta}$ similarly.

Define the open subscheme ${ }_{L} \check{Z}_{\max }^{\theta, r s s} \subset{ }_{L} \check{Z}^{\theta}$ and the gerb ${ }_{L} \check{\tilde{Z}}_{\max }^{\theta, r s s}$ similarly. As above, we get a trivialization of $\overline{\mathcal{L}}$ over ${ }_{L} \check{Z}_{\text {max }}^{\theta, r s s}$, hence an isomorphism

$$
{ }_{L} \check{Z}_{\max }^{\theta, r s s} \times B\left(\mu_{n}\right) \stackrel{\Im}{\rightarrow}_{L} \check{\tilde{Z}}_{\max }^{\theta, r s s}
$$


Let IC(Sign) denote the IC-sheaf of ${ }_{L} \check{Z}_{\max }^{\theta, r s s}$ tensored by the inverse image of Sign from $X^{\theta, r s s}$. Define $\mathrm{IC}_{L} \check{Z}^{\theta}, \bar{\zeta}$ as the intermediate extension of $\mathrm{IC}(\operatorname{Sign}) \otimes \mathcal{L}_{\bar{\zeta}^{-1}}$ to ${ }_{L} \tilde{\check{Z}}^{\theta}$ using (46). Now the isomorphism (43) yields an isomorphism

$$
\operatorname{Four}_{\psi}\left(\mathrm{IC}_{L} Z^{\theta}, \bar{\zeta}\right) \widetilde{\rightarrow} \mathrm{C}_{0}^{m} \otimes \mathrm{IC}_{L} \check{Z}^{\theta}, \bar{\zeta}
$$

The schemes ${ }_{L} Z^{\theta}$ for various $L$ are locally isomorphic in Zariski topology, so the description

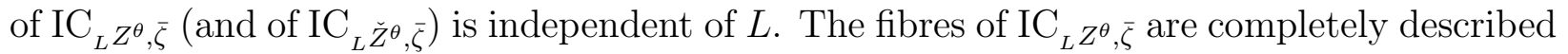
by Corollary 5.1 .

As $L$ varies in $\operatorname{Bun}_{1}$, the schemes ${ }_{L} Z^{\theta}$ form a family ${ }_{\mathrm{Bun}_{T}} Z^{\theta} \rightarrow \operatorname{Bun}_{1} \times X^{\theta}$, whose fibre over $(L, D)$ is $L(D) / L$. We still denote by $\overline{\mathcal{L}}$ the line bundle over $\operatorname{Bun}_{1} \times X^{\theta}$ with fibre (44) over $(L, D)$. Denote by $\operatorname{Bun}_{T} \widetilde{Z}^{\theta}$, Bun $_{T} \check{Z}^{\theta}$ and ${ }_{\operatorname{Bun}_{T}} \check{\tilde{Z}}^{\theta}$ the corresponding relative versions over $\operatorname{Bun}_{T}$.

We have an automorphism $\tau_{Z}$ of $\operatorname{Bun}_{T} \times X^{\theta}$ sending $(L, D)$ to $\left(L^{\prime}:=L^{-1} \otimes \Omega(-D), D\right)$. It lifts to a diagram of isomorphisms

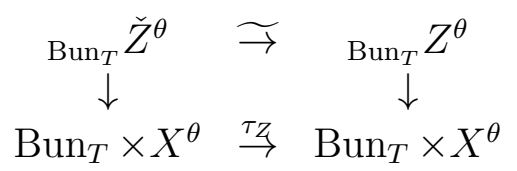

sending $\left(L, D, v \in L^{-1} \otimes \Omega / L^{-1} \otimes \Omega(-D)\right)$ to $\left(L^{\prime}, D, v \in L^{\prime}(D) / L^{\prime}\right)$, where $L^{\prime}$ is as above. Let $\triangle \subset X^{\theta}$ denote the divisor of diagonals. One has canonically $\overline{\mathcal{L}} \otimes \tau_{Z}^{*}(\overline{\mathcal{L}}) \widetilde{\rightarrow} \operatorname{pr}_{2}^{*} \mathcal{O}(-\triangle)$, where $\operatorname{pr}_{2}: \operatorname{Bun}_{T} \times X^{\theta} \rightarrow X^{\theta}$ is the projection. Recall that $\mathcal{O}(-\triangle)$ identifies canonically with the line bundle whose fibre at $D \in X^{\theta}$ is $\operatorname{det} \operatorname{R} \Gamma\left(X, \mathcal{O}_{D}\right)^{2}$. For $n=2$ this yields an isomorphisms

$$
\bar{\tau}_{Z}: \operatorname{Bun}_{T} \check{\tilde{Z}}^{\theta} \widetilde{\rightrightarrows}_{\mathrm{Bun}_{T}} \tilde{Z}^{\theta}
$$

and

$$
\bar{\tau}_{Z}^{*} \mathrm{IC}_{\mathrm{Bun}_{T} Z^{\theta}, \bar{\zeta}} \underset{\rightarrow}{\rightarrow} \mathrm{IC}_{\mathrm{Bun}_{T} \check{Z}^{\theta}, \bar{\zeta}}
$$

for the corresponding relative versions. Thus, for $n=2$ the description of $\mathrm{IC}_{L} \check{Z}^{\theta}, \bar{\zeta}$ is reduced to that of $\mathrm{IC}_{L} Z^{\theta}, \bar{\zeta}$, it was studied in [18]. However, for $n \geq 3$ the situation is very different.

For the rest of Section 7.2 .3 assume $L=\Omega$. Then $\overline{\mathcal{L}}$ is canonically identified with $\mathcal{O}(-\triangle)$ over $X^{\theta}$. Let $\Omega \tilde{X}^{\theta}$ be the gerb of $n$-th roots of $\overline{\mathcal{L}}$ over $X^{\theta}$. The fibre of ${ }_{\Omega} \check{Z}^{\theta} \rightarrow X^{\theta}$ over $D \in X^{\theta}$ is $\mathcal{O}_{D}$. Let

$$
\pi_{n}: \check{Z}_{\Omega} \check{Z}_{\Omega} \check{Z}^{\theta}
$$

be the map sending $\left(D, v \in \mathcal{O}_{D}\right)$ to $\left(D, v^{n} \in \mathcal{O}_{D}\right)$. Over ${ }_{\Omega} \check{Z}_{\text {max }}^{\theta}$ this map is finite. Let $\mathbb{G}_{n}^{\theta}$ denote the kernel of the homomorphism $\mathbb{G}^{\theta} \rightarrow \mathbb{G}^{\theta}, g \mapsto g^{n}$. This is a group scheme over $X^{\theta}$. Let $\mathbb{G}_{n}^{\theta}$ act on ${ }_{\Omega} \check{Z}^{\theta}$ so that $g \in(\mathcal{O} / \mathcal{O}(-D))^{*}$ sends $\left(D, v \in \mathcal{O}_{D}\right)$ to $(D, g v)$. The map $\pi_{n}$ is $\mathbb{G}_{n}^{\theta}$-invariant. The restriction

$$
\pi_{n}: \check{Z}_{\max }^{\theta} \rightarrow \Omega \check{Z}_{\max }^{\theta}
$$

is a $\mathbb{G}_{n}^{\theta}$-torsor. We have the line bundle on $X^{\theta}$ with fibre $\operatorname{det} \mathrm{R} \Gamma\left(X, \mathcal{O}_{D}\right)$, the group scheme $\mathbb{G}_{n}^{\theta}$ acts on this line bundle by a character that we denote $\eta_{n}: \mathbb{G}_{n}^{\theta} \rightarrow \mathbb{G}_{m}$. It actually takes 
values in $\mu_{n}$. Let $\check{W}_{\max }$ denote the local system on ${ }_{\Omega} \check{Z}_{\max }^{\theta}$ obtained from the torsor (49) as the extension of scalars via $\mathbb{G}_{n}^{\theta} \stackrel{\eta_{n}}{\rightarrow} \mu_{n}(k) \stackrel{\bar{\zeta}}{\rightarrow} \overline{\mathbb{Q}}_{\ell}^{*}$.

Let $\tilde{X}^{\theta, r s s}$ denote the restriction of the gerb ${ }_{\Omega} \tilde{X}^{\theta}$ to $X^{\theta, r s s}$. Since $\overline{\mathcal{L}}$ over $X^{\theta, r s s}$ is canonically trivialized, one has a canonical isomorphism

$$
X^{\theta, r s s} \times B\left(\mu_{n}\right) \widetilde{\rightrightarrows}_{\Omega} \tilde{X}^{\theta, r s s}
$$

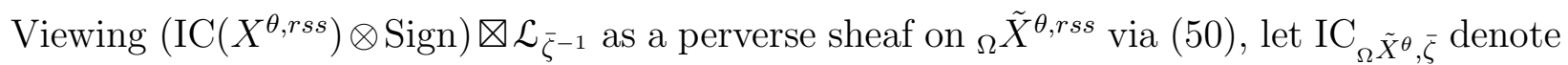
its intermediate extension to ${ }_{\Omega} \tilde{X}^{\theta}$. Let $\check{\pi}:{ }_{\Omega} \tilde{\tilde{Z}}^{\theta} \rightarrow{ }_{\Omega} \tilde{X}^{\theta}$ denote the projection sending $(D, v \in \mathcal{O} / \mathcal{O}(-D), \mathcal{U})$ to $(D, \mathcal{U})$.

Proposition 7.1. There is an isomorphism over ${ }_{\Omega} \check{\tilde{Z}}_{\max }^{\theta}$

$$
\check{W}_{\text {max }} \otimes \check{\pi}^{*} \mathrm{IC}_{\Omega \tilde{X}^{\theta}, \bar{\zeta}}[\operatorname{dim} \cdot \operatorname{rel}(\check{\pi})] \stackrel{\rightrightarrows}{\rightarrow} \mathrm{IC}_{\Omega} \check{Z}^{\theta}, \bar{\zeta}
$$

Proof The intermediate extension commutes with a smooth base change. So, it suffices to establish this isomorphism over ${ }_{\Omega} \tilde{Z}_{\text {max }}^{\theta, r s s}$, where it is easy.

Remark 7.1. The restriction of $\overline{\mathcal{L}}$ to the principal diagonal $X \subset X^{\theta}$ identifies with $\Omega^{m(m-1)}$. For $x \in X$ the group $\operatorname{Aut}\left(\Omega_{x}\right)$ acts on the fibre of $\overline{\mathcal{L}}$ at $D=m x$. So, if the $*$-fibre of $\mathrm{IC}_{\Omega} \tilde{X}^{\theta}, \bar{\zeta}$ at $m x$ does not vanish then $n$ divides $m(m-1)$. In particular, if $n$ is big enough compared to $m$ then $\mathrm{IC}_{\Omega} \tilde{X}^{\theta}, \bar{\zeta}$ is the extension by zero under ${ }_{\Omega} \tilde{X}^{\theta, r s s} \hookrightarrow{ }_{\Omega} \tilde{X}^{\theta}$.

Remark 7.2. i) The perverse sheaf $\mathrm{IC}_{\Omega} \tilde{X}^{\theta}, \bar{\zeta}$ has been studied in [1] (see also [10], Section 5.1). It satisfies the natural factorization property, and all its fibres are described in [1]. The version of $\mathrm{IC}_{\Omega} \tilde{X}^{\theta}, \bar{\zeta}$ in the world of twisted $\mathcal{D}$-modules is exactly the sheaf denoted by $\mathcal{L}_{\emptyset}^{\mu}$ in ([12], Section 3.4) for $G=\mathrm{SL}_{2}, \mu=-m \alpha$.

ii) The perverse sheaf $\mathrm{IC}_{\Omega} \tilde{X}^{\theta}, \bar{\zeta}$ also appears in [22] under the name $\mathcal{L}_{\emptyset}^{\mu}$ for $G=\mathrm{SL}_{2}, \mu=-m \alpha$. Note that for $n>1$ the so-called subtop cohomology property is satisfied for our metaplectic data for $\mathrm{SL}_{2}$ by $\left([22]\right.$, Theorem 1.1.6). So, for $n>1$ the perverse sheaf $\mathrm{IC}_{\Omega} \tilde{X}^{\theta}, \bar{\zeta}$ identifies with the !-direct image under ${ }_{\Omega} \widetilde{Z}^{\theta} \rightarrow{ }_{\Omega} \tilde{X}^{\theta}$ by ([22], Proposition 4.11.1). Here ${ }_{\Omega} \widetilde{Z}^{\theta}$ is the stack from Section 7.2.3.

7.2.4. For any $\mathcal{E} \in \operatorname{Bun}_{1}$ taking $L=\mathcal{E}^{2}$ we get $Z_{\mathcal{\varepsilon}}^{\theta}={ }_{L} Z^{\theta}$ and $\tilde{Z}_{\mathcal{\varepsilon}}^{\theta}={ }_{L} \tilde{Z}^{\theta}$. For $L=\mathcal{E}^{2}$ set $\mathrm{IC}_{\check{Z}_{c}^{\theta}, \bar{\zeta}}=\mathrm{IC}_{L} \check{Z}^{\theta}, \bar{\zeta}$. By (47) one has

$$
\operatorname{Four}_{\psi}\left(\mathrm{IC}_{Z_{c}^{\theta}, \bar{\zeta}}\right) \widetilde{\rightarrow} \mathrm{C}_{0}^{m} \otimes \mathrm{IC}_{\check{Z}_{c}^{\theta}, \bar{\zeta}}
$$

7.2.5. Global calculation. Let $\mathcal{S}_{B}$ denote the stack classifying $\mathcal{E} \in \operatorname{Bun}_{1}$ and $s_{2}: \mathcal{E}^{2} \rightarrow \Omega$. Let $\nu_{B}: \operatorname{Bun}_{B} \rightarrow \operatorname{Bun}_{G}$ be the natural map. Let $\operatorname{Bun}_{\tilde{B}, c}$ be the stack classifying (38) and a line $\mathcal{U}_{G}$ equipped with

$$
\mathcal{U}_{G}^{n} \widetilde{\Im}\left(\mathcal{L}_{c}\right)_{\mathcal{E}}
$$


Let $\mathcal{S}_{\tilde{B}, c}$ be the stack classifying $\left(\mathcal{E}, s_{2}\right) \in \mathcal{S}_{B}$, and a line $\mathcal{U}_{G}$ equipped with (52). We have the Fourier transform

$$
\operatorname{Four}_{\psi}: \mathrm{D}\left(\operatorname{Bun}_{\tilde{B}, c}\right) \widetilde{\rightarrow} \mathrm{D}\left(\mathcal{S}_{\tilde{B}, c}\right)
$$

The map $\nu_{B}$ lifts to a map $\nu_{\tilde{B}}: \operatorname{Bun}_{\tilde{B}, c} \rightarrow \widetilde{\operatorname{Bun}}_{G, \mathcal{L}_{c}}$ sending $\mathcal{U}_{G}$ and (38) to $\left(M, \mathcal{U}_{G}\right)$.

Recall that $Z_{\mathrm{Bun}_{T}}^{\theta}$ classifies $D \in X^{\theta}, \mathcal{E} \in \mathrm{Bun}_{1}, v \in \mathcal{E}^{2}(D) / \mathcal{E}^{2}$ giving rise to the diagram (41). We have the dual vector bundles $Z_{\mathrm{Bun}_{T}}^{\theta} \rightarrow X^{\theta} \times \operatorname{Bun}_{T} \leftarrow \check{Z}_{\mathrm{Bun}_{T}}^{\theta}$. Let

$$
f_{B}: Z_{\mathrm{Bun}_{T}}^{\theta} \rightarrow \operatorname{Bun}_{B} \times X^{\theta}
$$

be the map sending (41) to the exact sequence (38) together with $D \in X^{(m)}=X^{\theta}$. This is a morphism of generalized vector bundles over $\operatorname{Bun}_{T} \times X^{\theta}$ given by $\mathcal{E}^{2}(D) / \mathcal{E}^{2} \rightarrow \mathrm{H}^{1}\left(X, \mathcal{E}^{2}\right)$. The dual map over $\operatorname{Bun}_{T} \times X^{\theta}$ is denoted

$$
\check{f}_{B}: \mathcal{S}_{B} \times X^{\theta} \rightarrow \check{Z}_{\mathrm{Bun}_{T}}^{\theta}
$$

it sends $\left(\mathcal{E}, s_{2}, D\right)$ to $\left(D, \mathcal{E}, v \in \mathcal{E}^{-2} \otimes \Omega / \mathcal{E}^{-2} \otimes \Omega(-D)\right)$, where $v$ is the image of $s_{2}$ under the transpose map

$$
\mathrm{H}^{0}\left(X, \mathcal{E}^{-2} \otimes \Omega\right) \rightarrow \mathcal{E}^{-2} \otimes \Omega / \mathcal{E}^{-2} \otimes \Omega(-D)
$$

Let $\mathcal{S}_{B}^{0} \subset \mathcal{S}_{B}$ be the open substack given by $s_{2} \neq 0$. Let $\mathcal{S}_{\tilde{B}, c}^{0}$ be its preimage in $\mathcal{S}_{\tilde{B}, c}$. Let $\widetilde{\operatorname{Bun}}_{T, c}$ be the gerb of $n$-th roots of $\mathcal{L}_{c}$ over $\operatorname{Bun}_{T}$. For $K \in \mathrm{D}_{\bar{\zeta}}\left(\widetilde{\operatorname{Bun}}_{T, c}\right)$ let us describe

$$
\left.\operatorname{Four}_{\psi} \nu_{\tilde{B}}^{*} \operatorname{Eis}(K)\left[\operatorname{dim} \cdot \operatorname{rel}\left(\nu_{\tilde{B}}\right)\right]\right|_{\delta_{\tilde{B}, c}^{0}}
$$

Write $\left(\mathcal{E}_{1} \hookrightarrow M, \mathcal{U}_{G}, \mathcal{U}\right)$ for a point of $\overline{\operatorname{Bun}}_{\tilde{B}, c}$, here $\mathcal{U}_{G}^{n} \widetilde{\rightrightarrows}\left(\mathcal{L}_{c}\right)_{M}$ and $\mathcal{U}^{n} \widetilde{\rightrightarrows}\left(\mathcal{L}_{c}\right)_{\varepsilon_{1}}$.

Denote by $\tilde{Z}_{\mathrm{Bun}_{T}}^{\theta}$ the stack classifying a point $\left(\mathcal{E}, D, v \in \mathcal{E}^{2}(D) / \mathcal{E}^{2}\right) \in Z_{\mathrm{Bun}_{T}}^{\theta}$ and a line $\overline{\mathcal{U}}$ equipped with

$$
\overline{\mathcal{U}}^{n} \underset{\rightarrow}{\widetilde{(}} \frac{\left(\mathcal{L}_{c}\right)_{\mathcal{E}(D)}}{\left(\mathcal{L}_{c}\right)_{\mathcal{E}}}
$$

Let $\check{\check{Z}}_{\mathrm{Bun}_{T}}^{\theta}$ be the stack classifying $\left(\mathcal{E}, D, v^{\prime} \in \mathcal{E}^{-2} \otimes \Omega / \mathcal{E}^{-2} \otimes \Omega(-D)\right) \in \check{Z}_{\mathrm{Bun}_{T}}^{\theta}$ and a line $\overline{\mathcal{U}}$ equipped with (54). The perverse sheaves $\mathrm{IC}_{Z_{c}^{\theta}, \bar{\zeta}}$ as $\mathcal{E}$ varies in $\mathrm{Bun}_{T}$ naturally form a family, which is a perverse sheaf on $\tilde{Z}_{\mathrm{Bun}_{T}}^{\theta}$ still denoted $\mathrm{IC}_{Z_{c}^{\theta}, \bar{\zeta}}$ by abuse of notations. Similarly, we denote by $\mathrm{IC}_{\check{Z}_{c}^{\theta}, \bar{\zeta}}$ the corresponding perverse sheaf over $\check{\tilde{Z}}_{\mathrm{Bun}_{T}}^{\theta}$. The isomorphism (51) naturally extends to the stack $\check{\check{Z}}_{\mathrm{Bun}_{T}}^{\theta}$.

Write $\left(\mathcal{S}_{B} \times X^{\theta}\right)^{\tilde{y}}$ for the stack classifying $\left(\mathcal{E}, s_{2}\right) \in \mathcal{S}_{B}$ and lines $\mathcal{U}_{G}, \mathcal{U}$ equipped with (52) and

$$
\mathcal{U}^{n} \widetilde{\rightrightarrows}\left(\mathcal{L}_{c}\right)_{\mathcal{E}(D)}
$$

The map $\check{f}_{B}$ extends to a morphism $\check{f}_{\tilde{B}}:\left(\mathcal{S}_{B} \times X^{\theta}\right)^{\tilde{}} \rightarrow \check{\tilde{Z}}_{\mathrm{Bun}_{T}}^{\theta}$ given by $\overline{\mathcal{U}}=\mathcal{U} \otimes \mathcal{U}_{G}^{-1}$. We have a diagram of projections

$$
\widetilde{\operatorname{Bun}}_{T, c} \stackrel{\operatorname{pr}_{T, c}}{\leftarrow}\left(\mathcal{S}_{B} \times X^{\theta}\right)^{\tilde{p}} \stackrel{\operatorname{pr}_{B, c}}{\rightarrow} \mathcal{S}_{\tilde{B}, c},
$$


where $\operatorname{pr}_{B, c}$ sends the above point to $\left(\mathcal{E}, s_{2}, \mathcal{U}_{G}\right)$, and the map $\operatorname{pr}_{T, c}$ sends the above point to $\left(\mathcal{E}^{-1}(-D), \mathcal{U}\right)$ equipped with (55). From the standard properties of the Fourier transform one gets the following.

Proposition 7.2. Let $m \geq 0, \theta=m \alpha$. Over the connected component of $\mathcal{S}_{\tilde{B}, c}^{0}$ given by fixing $\operatorname{deg} \mathcal{E}$, the contribution of the connected component of $\overline{\operatorname{Bun}}_{\tilde{B}, c}$ given by $\operatorname{deg} \mathcal{E}_{1}=-\operatorname{deg} \mathcal{E}-m$ to the complex (53) identifies with

$$
\left(\operatorname{pr}_{B, c}\right) !\left(\operatorname{pr}_{T, c}^{*} K \otimes \mathcal{C}_{0}^{m} \otimes\left(\check{f}_{\tilde{B}}\right)^{*} \mathrm{IC}_{\check{Z}_{c}^{\theta}, \bar{\zeta}}\right)\left[\operatorname{dim} \cdot \operatorname{rel}\left(\check{f}_{B}\right)-\operatorname{dim} \operatorname{Bun}_{T}\right]
$$

This complex vanishes unless e divides $m+\operatorname{deg} \mathcal{E}$.

Proposition 7.2 implies the following description of the first Whittaker coefficients of $\operatorname{Eis}(K), K \in \mathrm{D}_{\bar{\zeta}}\left(\widetilde{\operatorname{Bun}}_{T, c}\right)$. Write $\operatorname{Cov}_{B} \subset \mathcal{S}_{B}$ for the open substack given by requiring that $s_{2}: \mathcal{E}^{2} \rightarrow \Omega$ is an isomorphism. Let $\operatorname{Cov}_{\tilde{B}, c}$ denote the restriction of the gerb $\mathcal{S}_{\tilde{B}, c}$ to $\operatorname{Cov}_{B}$. The stack $\operatorname{Cov}_{\tilde{B}, c}$ is the base, on which the first Whittaker coefficient lives. Recall that the line bundle on $X^{\theta} \times \operatorname{Cov}_{B}$ whose fibre at $\left(D, \mathcal{E}, s_{2}\right) \in X^{\theta} \times \operatorname{Cov}_{B}$ is

$$
\frac{\left(\mathcal{L}_{c}\right)_{\mathcal{E}(D)}}{\left(\mathcal{L}_{c}\right)_{\mathcal{E}}}
$$

identifies canonically with $\operatorname{pr}_{1}^{*} \mathcal{O}(-\triangle)$. So, one gets the open immersion $\operatorname{Cov}_{\tilde{B}, c} \times\left({ }_{\Omega} \tilde{X}^{\theta}\right) \hookrightarrow$ $\left(\mathcal{S}_{B} \times X^{\theta}\right)^{\tilde{s}}$ sending $\left(D, \mathcal{E}, s_{2}, \mathcal{U}_{G}, \overline{\mathcal{U}}\right)$ with (52) and (54) to $\left(\mathcal{E}, s_{2}, D, \mathcal{U}_{G}, \mathcal{U}\right)$, where $\mathcal{U}=\mathcal{U}_{G} \otimes \overline{\mathcal{U}}$. We get the diagram

obtained by restricting (56).

$$
\widetilde{\operatorname{Bun}}_{T, c} \stackrel{\operatorname{pr}_{\text {Cov }}, \theta}{\leftarrow} \operatorname{Cov}_{\tilde{B}, c} \times\left({ }_{\Omega} \tilde{X}^{\theta}\right) \stackrel{\operatorname{pr}_{1}}{\rightarrow} \operatorname{Cov}_{\tilde{B}, c}
$$

Corollary 7.1. The restriction of (57) to $\mathrm{Cov}_{\tilde{B}, c}$ identifies with

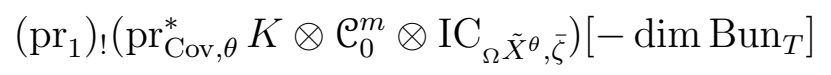

Corollary 7.2. Let $E$ be a $\check{T}^{\sharp}$-local system on $X, \mathcal{K}_{E} \in \mathrm{D}_{\zeta}\left(\widetilde{\operatorname{Bun}}_{T}\right)$ be the $E^{*}$-Hecke eigensheaf as in Corollary 2.1. The first Whittaker coefficient of $\operatorname{Eis}\left(\mathcal{K}_{E}\right)$, that is, the complex

$$
\left.\operatorname{Four}_{\psi} \nu_{\tilde{B}}^{*} \operatorname{Eis}\left(\mathcal{K}_{E}\right)\left[\operatorname{dim} . \operatorname{rel}\left(\nu_{\tilde{B}}\right)\right]\right|_{\operatorname{Cov}_{\tilde{B}, c}}
$$

identifies with

$$
\oplus_{\theta}\left(\operatorname{pr}_{1}\right) !\left(\operatorname{pr}_{\mathrm{Cov}, \theta}^{*} \mathcal{K}_{E} \otimes \mathcal{C}_{0}^{m} \otimes \mathrm{IC}_{\Omega} \tilde{X}^{\theta}, \bar{\zeta}\right)
$$

Here $\theta=m \alpha$, and the sum is over $m \geq 0$ such that $m+g-1 \in e \mathbb{Z}$.

Remark 7.3. i) The complex (58) is an $\ell$-adic analog of the space of conformal blocks in Wess-Zumino-Witten model that was studied in [1].

ii) Assume $E^{n \alpha}$ nontrivial then $\mathcal{K}_{E}$ is regular. The conjectural functional equation for Eis $\left(\mathcal{K}_{E}\right)$ should be reflected in the property of (59) saying that the summand indexed by $\theta=m \alpha$ for $E$ identifies (up to tensoring by some 1-dimensional space) with the summand indexed by $\theta^{\prime}=m^{\prime} \alpha$ for ${ }^{\sigma} E$, where $m+m^{\prime}=(n-1)(2 g-2)$. Here ${ }^{\sigma} E$ is the extension of 
scalars of $E$ under $w_{0}: \check{T}^{\sharp} \rightarrow \check{T}^{\sharp}$. In particular, in this case the sum in (59) should be over $0 \leq m \leq(n-1)(2 g-2)$ such that $m+g-1 \in e \mathbb{Z}$.

iii) In the case $n=2$ the complex (59) is calculated in ([18], Theorem 4). In this case it is given by the 'geometric central value' of some $L$-function. Recall the line bundle $\mathcal{E}_{X} \in \operatorname{Cov}_{B}$ from Section 2.0.3. A point $\left(\varepsilon, s_{2}\right) \in \operatorname{Cov}_{B}$ gives rise to the $\mu_{2}$-torsor on $X$ given by $s_{2}:\left(\mathcal{E} \otimes \mathcal{E}_{X}^{-1}\right)^{2} \widetilde{\Im} \mathcal{O}_{X}$. Write $\mathcal{E}_{0}$ for the $\overline{\mathbb{Q}}_{\ell}$-local system on $X$ obtained from this $\mu_{2}$-torsor via the extension $\mu_{2} \rightarrow \overline{\mathbb{Q}}_{\ell}^{*}$. For $n=2$ the fibre of (59) over $\left(\mathcal{E}, \mathcal{U}_{G}\right) \in \operatorname{Cov}_{\tilde{B}, c}$ identifies with

$$
\oplus_{m \geq 0} \mathrm{R} \Gamma\left(X^{(m)},\left(E^{-\alpha} \otimes \mathcal{E}_{0}\right)^{(m)}\right)[m]
$$

tensored by some 1-dimensional space. If $E^{-2 \alpha}$ is nontrivial then the above identifies with

$$
\underset{0 \leq m \leq 2 g-2}{\oplus} \wedge^{m} \mathrm{H}^{1}\left(X, E^{-\alpha} \otimes \mathcal{E}_{0}\right)
$$

tensored by some 1-dimensional space, and this agrees with ii). The compatibility with the functional equation then comes from the isomorphism $\mathrm{H}^{1}\left(X, E^{-\alpha} \otimes \varepsilon_{0}\right)^{*} \underset{\rightarrow}{\rightarrow} \mathrm{H}^{1}\left(X, E^{\alpha} \otimes \varepsilon_{0}\right)$. So, for $n>1$ we may think of (159) as a generalization of the notion of the central value of an abelian $L$-function.

7.3. Constant term of Eis. Recall that $\operatorname{Bun}_{B, \tilde{G}}$ classifies an exact sequence (38) and a line $\mathcal{U}_{G}$ equipped with $\mathcal{U}_{G}^{N} \underset{\rightarrow}{\rightarrow} \mathcal{L}_{M}$. Write $\operatorname{Bun}_{B, \tilde{G}}^{d}$ for the connected component of $\mathrm{Bun}_{B, \tilde{G}}$ given by $\operatorname{deg} \mathcal{E}=d$. We have the diagram of projections

$$
\widetilde{\operatorname{Bun}}_{T} \stackrel{\mathfrak{q}}{\leftarrow} \operatorname{Bun}_{B, \tilde{G}} \stackrel{\mathfrak{p}}{\rightarrow} \widetilde{\operatorname{Bun}}_{G},
$$

where $\mathfrak{q}$ sends $\left(\mathcal{E} \hookrightarrow M, \mathcal{U}_{G}\right)$ to $\left(\mathcal{E}, \mathcal{U}_{G}\right)$. Write $\widetilde{\operatorname{Bun}}_{T}^{d}$ for the component of $\widetilde{\operatorname{Bun}}_{T}$ classifying $(\mathcal{E}, \mathcal{U}) \in \widetilde{\operatorname{Bun}}_{T}$ with $\operatorname{deg} \mathcal{E}=d$. The constant term functor $\mathrm{CT}: \mathrm{D}_{\zeta}\left(\widetilde{\operatorname{Bun}}_{G}\right) \rightarrow \mathrm{D}_{\zeta}\left(\widetilde{\operatorname{Bun}}_{T}\right)$ is defined by $\mathrm{CT}=\mathfrak{q} ! \mathfrak{p}^{*}[\operatorname{dim} . \operatorname{rel}(\mathfrak{p})]$.

Recall that $\overline{\operatorname{Bun}}_{B}$ classifies $M \in \operatorname{Bun}_{G}$ together with a subsheaf $\mathcal{E} \hookrightarrow M, \mathcal{E} \in \operatorname{Bun}_{1}$. Write $\overline{\operatorname{Bun}}_{B}^{d}$ for the connected component of $\overline{\mathrm{Bun}}_{B}$ given by $\operatorname{deg} \mathcal{E}=d$. The stack $\overline{\mathrm{Bun}}_{B}^{d}$ is smooth irreducible of dimension $2 g-2-2 d$.

Let $\sigma: \operatorname{Bun}_{T} \rightarrow \operatorname{Bun}_{T}$ be the map $\mathcal{E} \mapsto \mathcal{E}^{-1}$. We also denote by $\sigma: \widetilde{\operatorname{Bun}}_{T} \rightarrow \widetilde{\operatorname{Bun}}_{T}$ the $\operatorname{map}(\mathcal{E}, \mathcal{U}) \mapsto\left(\mathcal{E}^{-1}, \mathcal{U}\right)$.

Definition 7.1. For $\theta=m \alpha \in \Lambda_{G, B}^{\text {pos }}$ with $m \in n \mathbb{Z}_{+}$we define the following integral Hecke functor $\mathrm{IH}^{\theta}: \mathrm{D}_{\zeta}\left(\widetilde{\operatorname{Bun}}_{T}\right) \rightarrow \mathrm{D}_{\zeta}\left(\widetilde{\operatorname{Bun}}_{T}\right)$. One has $\mathfrak{B}(\theta)=\frac{m}{n} \nu$, where $\nu=n \alpha \in J$, and $X^{\mathfrak{B}(\theta)} \underset{\rightrightarrows}{\rightarrow} X^{\left(\frac{m}{n}\right)}$. Recall the stack $X^{\mathfrak{B}(\theta)} \times_{X^{\theta}} \widetilde{\operatorname{Mod}}_{\mathrm{Bun}_{T}}^{+, \theta}$ from Section 17.1.1, Its point is a collection $\left(\mathcal{E} \in \operatorname{Bun}_{1}, D \in X^{\left(\frac{m}{n}\right)}, \mathcal{U}, \mathcal{U}_{G}\right)$ together with isomorphisms $\mathcal{U}_{G}^{N} \widetilde{\Im}\left(\mathcal{L}_{T}\right)_{\mathcal{E}(n D)}$, $\mathcal{U}^{N} \widetilde{\rightrightarrows}\left(\mathcal{L}_{T}\right)_{\mathcal{E}}$. Here $\mathcal{E}(n D)$ is the 'background' $T$-torsor. Consider the diagram

$$
\widetilde{\operatorname{Bun}}_{T} \stackrel{h_{T}^{\overleftarrow{T}}}{\leftarrow} X^{\mathfrak{B}(\theta)} \times_{X^{\theta}} \widetilde{\operatorname{Mod}_{\overparen{\operatorname{Bun}_{T}}}^{+, \theta}} \stackrel{h_{T}}{\rightarrow} \widetilde{\operatorname{Bun}}_{T},
$$

where $h_{T}^{\overleftarrow{T}}$ sends the above point to $(\mathcal{E}, \mathcal{U})$, and $h_{T}^{\rightarrow}$ sends the above point to $\left(\mathcal{E}(n D), \mathcal{U}_{G}\right)$ Set

$$
\mathrm{IH}^{\theta}(K)=\left(h_{T}^{\rightarrow}\right) !\left(\left(h_{T}^{\leftarrow}\right)^{*} K \otimes \mathrm{IC}_{\mathrm{Bun}_{M}, \zeta}^{\mathfrak{B}(\theta)}\right)\left[-\operatorname{dim} \operatorname{Bun}_{T}\right]
$$


Proposition 7.3. Let $d_{1} \in e \mathbb{Z}$ and $K \in \mathrm{D}_{\zeta}\left(\widetilde{\operatorname{Bun}}_{T}^{d}\right)$. The complex $K_{d, d_{1}}:=\left.\mathrm{CT}(\operatorname{Eis}(K))\right|_{\widetilde{\operatorname{Bun}}_{T}}$ vanishes unless $d-d_{1} \in n \mathbb{Z}$. In the latter case it is described as follows.

1) If $d_{1}>\max \{d,-d\}$ then $K_{d, d_{1}}=0$.

2) If $d<d_{1} \leq-d$ then for $\theta:=-\left(d+d_{1}\right) \alpha \in n \alpha \mathbb{Z}$

$$
K_{d, d_{1}} \widetilde{\rightarrow} \sigma_{!} \mathrm{IH}^{\theta}(K)[-|\mathfrak{B}(\theta)|]
$$

3) If $d \geq d_{1}>-d$ then for $\theta:=\left(d-d_{1}\right) \alpha \in n \alpha \mathbb{Z}$

$$
K_{d, d_{1}} \stackrel{\rightarrow}{\rightarrow} \mathrm{IH}^{\theta}(K)[2-2 g+|\mathfrak{B}(\theta)|]
$$

4) If $d_{1} \leq \min \{d,-d\}$ then there is a distinguished triangle

$$
\sigma_{!} \mathrm{IH}^{\theta}(K)[-|\mathfrak{B}(\theta)|] \rightarrow K_{d, d_{1}} \rightarrow \mathrm{IH}^{\theta^{\prime}}(K)\left[2-2 g+\left|\mathfrak{B}\left(\theta^{\prime}\right)\right|\right]
$$

with $\theta=-\left(d+d_{1}\right) \alpha$ and $\theta^{\prime}=\left(d-d_{1}\right) \alpha$.

Proof We calculate the direct image with respect to the composition $\operatorname{Bun}_{B, \tilde{G}} \times{\widetilde{\mathrm{Bun}_{G}}}_{\overline{\mathrm{Bun}}_{\tilde{B}}} \stackrel{\tilde{\mathfrak{p}}}{\rightarrow}$ $\operatorname{Bun}_{B, \tilde{G}} \stackrel{\mathfrak{q}}{\rightarrow} \widetilde{\operatorname{Bun}}_{T}$. Write a point of $\operatorname{Bun}_{B, \tilde{G}} \times_{\widetilde{\operatorname{Bun}}_{G}} \overline{\operatorname{Bun}}_{\tilde{B}}$ as (38) together with a subsheaf $\mathcal{E}_{1} \hookrightarrow M$ and lines $\mathcal{U}, \mathcal{U}_{G}$ equipped with $\mathcal{U}^{N} \widetilde{\Im}\left(\mathcal{L}_{T}\right)_{\varepsilon_{1}}, \mathcal{U}_{G}^{N} \Im\left(\mathcal{L}_{T}\right)_{\varepsilon}$.

1) In this case $\operatorname{Hom}\left(\varepsilon_{1}, \mathcal{E}\right)=\operatorname{Hom}\left(\mathcal{E}_{1}, \mathcal{E}^{-1}\right)=0$.

2) In this case $\operatorname{Hom}\left(\varepsilon_{1}, \mathcal{E}\right)=0$, and there remains the integral over the open substack $\tilde{Z}_{\widetilde{\operatorname{Bun}}_{T}^{\theta}}^{\theta} \subset \operatorname{Bun}_{B, \tilde{G}} \times{\widetilde{\operatorname{Bun}_{G}}}_{\operatorname{Bun}_{\tilde{B}}}$ given by the conditions that $\mathcal{E}_{1} \rightarrow \mathcal{E}^{-1}$ is injective, deg $\mathcal{E}_{1}=d_{1}$, $\operatorname{deg} \mathcal{E}=d$. Here $\theta=-\left(d+d_{1}\right) \alpha$.

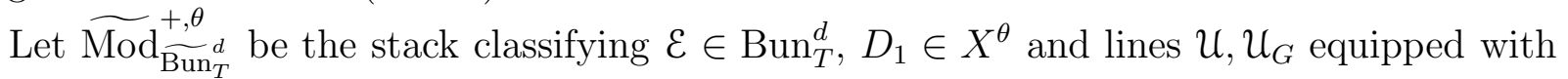
$\mathcal{U}^{N} \widetilde{\rightarrow}\left(\mathcal{L}_{T}\right)_{\mathcal{E}\left(D_{1}\right)}, \mathcal{U}_{G}^{N} \widetilde{\rightrightarrows}\left(\mathcal{L}_{T}\right)_{\mathcal{E}}$. Here $\mathcal{E}^{-1}$ is the 'background' $T$-torsor. Let $\pi_{B}: \tilde{Z}_{\widetilde{\mathrm{Bun}}_{T}}^{\theta} \rightarrow$

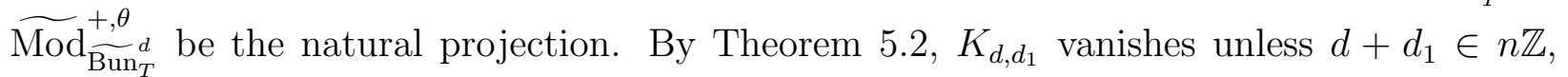
and in the latter case it is as follows. One gets $X^{\mathfrak{B}(\theta)}=X^{\left(\frac{-d-d_{1}}{n}\right)}$. The corresponding map $X^{\mathfrak{B}(\theta)} \rightarrow X^{\theta}$ sends $D$ to $D_{1}=n D$. Consider the diagram

$$
\widetilde{\operatorname{Bun}_{T}} \stackrel{h_{T}^{\leftarrow}}{\leftarrow} X^{\mathfrak{B}(\theta)} \times_{X^{\theta}} \widetilde{\operatorname{Mod}_{\overparen{\operatorname{Bun}}}} \stackrel{+, \theta}{\stackrel{\sigma h_{F}}{\rightarrow}} \widetilde{\operatorname{Bun}_{T}},
$$

where $h_{T}^{\rightarrow}$ sends $\left(D, \mathcal{E}, \mathcal{U}, \mathcal{U}_{G}\right)$ to $\left(\mathcal{E}^{-1}, \mathcal{U}_{G}\right)$, and $h_{T}^{\overleftarrow{T}}$ sends this point to $\left(\mathcal{E}^{-1}(-n D), \mathcal{U}\right)$. By Theorem 5.2 ,

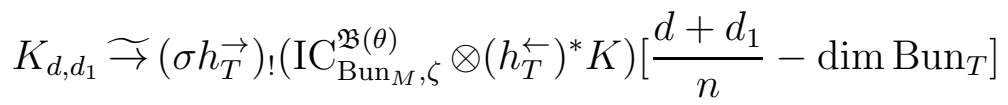

3) In this case $\operatorname{Hom}\left(\mathcal{E}_{1}, \mathcal{E}^{-1}\right)=0$. Let $\theta=\left(d-d_{1}\right) \alpha$. By Corollary 5.1, $K_{d, d_{1}}$ vanishes unless $d-d_{1} \in n \mathbb{Z}$. In the latter case $|\mathfrak{B}(\theta)|=\frac{d-d_{1}}{n}$ and

$$
K_{d, d_{1}} \underset{\rightarrow}{\rightarrow} \mathrm{IH}^{\theta}(K)[2-2 g+|\mathfrak{B}(\theta)|]
$$

4) Stratify $\operatorname{Bun}_{B, \tilde{G}} \times_{\widetilde{B u n}_{G}} \overline{\operatorname{Bun}}_{\tilde{B}}$ by the property that $\varepsilon_{1}$ factors through $\mathcal{E}$ or not. Calculate the direct image with respect to this stratifications. 
Let $E$ be a $\check{T}^{\sharp}$-local system on $X$. Write $\mathcal{K}=\mathcal{K}_{E}$ for the Hecke eigen-sheaf on $\widetilde{\operatorname{Bun}}_{T}$ associated to $E$ in ([17], Proposition 2.2). This is a local system over the components of $\widetilde{\operatorname{Bun}}_{T}$ corresponding to $\Lambda^{\sharp}$.

Lemma 7.2. Let $\nu=-n \alpha$ and $\theta=m \alpha$ with $m \in n \mathbb{Z}_{+}$. One has naturally $\operatorname{IH}^{\theta}\left(\mathcal{K}_{E}\right) \widetilde{\Im} \mathcal{K}_{E} \otimes$ $\mathrm{R} \Gamma\left(X^{\left(\frac{m}{n}\right)},\left(E^{\nu}\right)^{\left(\frac{m}{n}\right)}\right)\left[\frac{m}{n}\right]$.

Remark 7.4. By ([18], Lemma 15), one has canonically $(A E)_{\Omega^{-n \alpha}} \widetilde{\rightarrow} \operatorname{det} \mathrm{R} \Gamma\left(X, E^{n \alpha}\right)$. Here $\Omega^{-n \alpha}$ is the $T^{\sharp}$-torsor obtained from $\Omega$ via the push-out by $-n \alpha: \mathbb{G}_{m} \rightarrow T^{\sharp}$. Denote also by ${ }^{\sigma} E$ the extension of scalars of $E$ under $w_{0}: \check{T}^{\sharp} \rightarrow \check{T}^{\sharp}$. Now from Proposition 3.7 one gets $\sigma * \mathcal{K}_{E} \widetilde{\rightarrow} \mathcal{K}_{(\sigma E)} \otimes \operatorname{det} \operatorname{R\Gamma }\left(X, E^{n \alpha}\right)$. Here $\sigma *$ stands for the twisted $W$-action (15). It follows that Proposition 7.3 is consistent with the functional equation for $\operatorname{Eis}\left(\mathcal{K}_{E}\right)$ from Conjecture 3.1 .

7.4. Some special sheaves. Let $E$ be a $\check{T}^{\sharp}$-local system on $X$. Sometimes we think of it simply as the rank one local system on $X$ corresponding to the character e $\alpha$ of $\check{T}^{\sharp}$. Write $\mathcal{K}=\mathcal{K}_{E}$ for the Hecke eigen-sheaf on $\widetilde{\operatorname{Bun}}_{T}$ associated to $E$ in ([17], Proposition 2.2). This is a local system over the components of $\widetilde{\operatorname{Bun}}_{T}$ corresponding to $\Lambda^{\sharp}$.

Recall the Shatz stratification of $\operatorname{Bun}_{G}$. Let $S h a t z^{0} \subset \operatorname{Bun}_{G}$ be the open substack of semi-stable torsors. So, $M \in S h a t z^{0}$ iff for any rank one subsheaf $L \subset M$ one has $\operatorname{deg} L \leq 0$. For $d>0$ let $S h a t z^{d}$ denote the stack classifying $\mathcal{E} \in \operatorname{Bun}_{1}^{d}$ and an exact sequence $0 \rightarrow$ $\mathcal{E} \rightarrow M \rightarrow \mathcal{E}^{-1} \rightarrow 0$. The map $S h a t z^{d} \rightarrow \operatorname{Bun}_{G}$ sending this point to $M$ is a locally closed immersion. Besides, Shatz $z^{d}$ for $d \geq 0$ form a stratification of Bun ${ }_{G}$. The stack Shat $z^{d}$ is irreducible of dimension $2 g-2-2 d$ for $d>0$, and $\operatorname{dim} S h a t z^{0}=\operatorname{dim} \operatorname{Bun}_{G}=3 g-3$.

Let $\operatorname{Bun}_{G}^{s t} \subset \operatorname{Bun}_{G}$ denote the open substack of stable sheaves. It is not empty for $g \geq 2$. The image $\operatorname{Bun}_{\bar{G}}^{\leq 0}$ of $\overrightarrow{\operatorname{Bun}}_{B}^{0} \rightarrow \operatorname{Bun}_{G}$ is the complement of $\operatorname{Bun}_{G}^{s t}$ in $\operatorname{Bun}_{G}$. For $d>0$ the image of $\overline{\mathfrak{p}}: \overline{\operatorname{Bun}}_{B}^{d} \rightarrow \operatorname{Bun}_{G}$ is the closure $\overline{S h a t z}^{d}$ of $S h a t z^{d}$. Let $S h a t z_{\tilde{G}}^{d}$ (resp., $\overline{S h a t z}_{\tilde{G}}^{d}$ ) be obtained from $S h a t z_{G}^{d}$ (resp., $\overline{\text { Shatz }}^{d}$ ) by the base change $\widetilde{\operatorname{Bun}}_{G} \rightarrow \operatorname{Bun}_{G}$.

For $d>0$ a point of $S h a t z_{\tilde{G}}^{d}$ is given by (38) together with a line $\mathcal{U}_{G}$ equipped with

$$
\mathcal{U}_{G}^{N} \widetilde{\rightrightarrows}\left(\mathcal{L}_{T}\right)_{\mathcal{E}}
$$

For $d>0$ let $\tilde{q}: S h a t z_{\tilde{G}}^{d} \rightarrow \widetilde{\operatorname{Bun}}_{T}$ be the map sending the above point to $\left(\mathcal{E}, \mathcal{U}_{G}\right)$ equipped with (60).

Definition 7.2. Let $\operatorname{IC}(E, d)$ denote the intermediate extension of $\tilde{q}^{*} \mathcal{K}_{E}\left[\operatorname{dim} S h a t z_{\tilde{G}}^{d}\right]$ under $\operatorname{Shatz}_{\tilde{G}}^{d} \hookrightarrow \overline{\operatorname{Shatz}}_{\tilde{G}}^{d}$. Note that $\mathrm{IC}(E, d) \in \mathrm{D}_{\zeta}\left(\widetilde{\operatorname{Bun}}_{G}\right)$.

Recall the stack $\overline{\operatorname{Bun}}_{B}^{d}$ from Section 7.3 . For $K \in \mathrm{D}_{\zeta}\left(\widetilde{\operatorname{Bun}}_{T}\right)$ write $\operatorname{Eis}^{d}(K)$ for the contribution of the component $\overline{\operatorname{Bun}}_{\tilde{B}}^{d}$ to $\operatorname{Eis}(K)$. Recall that $\operatorname{Eis}^{d}(\mathcal{K})$ vanishes unless $d \in e \mathbb{Z}$.

Proposition 7.4. Let $d>0$ with $d \in e \mathbb{Z}$.

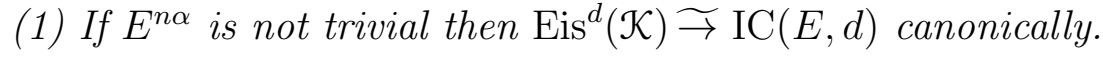


(2) If $E^{n \alpha}$ is trivial then

$$
\operatorname{Eis}^{d}(\mathcal{K}) \stackrel{\Im}{\rightarrow} \oplus_{b \geq 0} \quad \mathrm{IC}(E, d+n b)
$$

Proof The map $\overline{\mathfrak{p}}: \overline{\operatorname{Bun}}_{B}^{d} \rightarrow \operatorname{Bun}_{G}$ is an isomorphism over Shatz $z_{G}^{d}$. It follows that $\mathrm{IC}(E, d)$ appears in $\operatorname{Eis}^{d}(\mathcal{K})$ with multiplicity one. Consider a point $\left(\mathcal{E} \hookrightarrow M, \mathcal{U}_{G}\right) \in S h a t z_{\tilde{G}}^{r}$ for some $r>d$. The fibre of $\overline{\mathfrak{p}}: \overline{\operatorname{Bun}}_{B}^{d} \rightarrow \operatorname{Bun}_{G}$ over this point identifies with $X^{(r-d)}$. Namely, to $D \in X^{(r-d)}$ there corresponds the subsheaf $\mathcal{E}(-D) \subset M$. Denote by $S$ the $*$-fibre of $\operatorname{Eis}^{d}(\mathcal{K})$ at this point. By Corollary 5.1, $S$ vanishes unless $r-d \in n \mathbb{Z}$. If $r-d \in n \mathbb{Z}$ then we get an isomorphism

$$
S \widetilde{\rightarrow} \mathcal{K}_{\left(\varepsilon, \mathcal{U}_{G}\right)} \otimes \mathrm{R} \Gamma\left(X^{\left(\frac{r-d}{n}\right)},\left(E^{-n \alpha}\right)^{\left(\frac{r-d}{n}\right)}\right)\left[-2 r+2 g-2+\frac{2(r-d)}{n}\right]
$$

The codimension of $S h a t z^{r}$ in $\overline{S h a t z}^{d}$ is $2(r-d)$. If $E^{n \alpha}$ is not trivial then the $*$-restriction of $\operatorname{Eis}^{d}(\mathcal{K})$ to $S h a t z^{r}$ is placed in perverse degrees $<0$. Part $(1)$ follows. Under the assumption of $(2)$ we see that $\operatorname{IC}(E, r)$ appears in $\operatorname{Eis}^{d}(\mathcal{K})$ with multiplicity one. Our claim follows.

Remark 7.5. If $n$ is even then by Lemma 3.5 one has the following. If $d \in n \mathbb{Z}$ (resp., $d \in e \mathbb{Z}$ and $d \notin n \mathbb{Z})$ then $\mathrm{IC}(E, d) \in \mathrm{D}_{\zeta,+}\left(\widetilde{\operatorname{Bun}_{G}}\right)$ (resp., $\left.\mathrm{IC}(E, d) \in \mathrm{D}_{\zeta,-}\left(\widetilde{\operatorname{Bun}_{G}}\right)\right)$.

7.4.1. Case $g=0$. In this subsection we assume $g=0$. For $d>0$ set for brevity $\mathrm{IC}_{d}=$ $\operatorname{IC}\left(\overline{\mathbb{Q}}_{\ell}, d\right)$. The open substack $\operatorname{Shatz}^{0} \subset \operatorname{Bun}_{G}$ classifies trivial $G$-torsors.

Definition 7.3. The line bundle $\mathcal{L}_{c}$ is trivial on $S h a t z^{0}$, its trivialization yields an isomor-

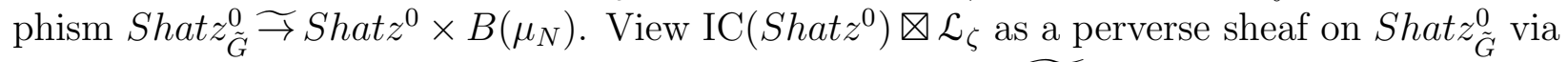
this isomorphism. Let $\mathrm{IC}_{0}$ be its intermediate extension to $\widetilde{\operatorname{Bun}}_{G}$.

Lemma 7.3. Assume $d>0$ with $d \in e \mathbb{Z}$. Let $r>d$. The $*$-restriction $\left.\mathrm{IC}_{d}\right|_{\text {Shat }}{ }_{\tilde{G}}$ vanishes unless $r-d \in n \mathbb{Z}$. If $r-d \in n \mathbb{Z}$ then

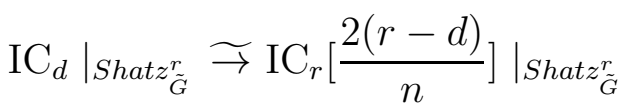

Proof By Proposition [7.4. $\operatorname{Eis}^{d}(\mathcal{K}) \widetilde{\rightarrow} \operatorname{Eis}^{d+n}(\mathcal{K}) \oplus \mathrm{IC}_{d}$. Restricting this isomorphism to Shat $z_{\tilde{G}}^{r}$, one obtains the desired result as in Proposition 7.4 .

Lemma 7.4. (1) One has $\operatorname{Eis}^{0}(\mathcal{K}) \widetilde{\rightarrow} \mathrm{IC}_{0}[1] \oplus \mathrm{IC}_{0}[-1] \oplus\left(\oplus_{b \geq 0} \mathrm{IC}_{2 n+b n}\right)$.

(2) The *-restriction $\left.\mathrm{IC}_{0}\right|_{\text {Shat }} \tilde{\tilde{G}}_{\tilde{G}}$ vanishes unless $r \in n \mathbb{Z}$. For $r \in n \mathbb{Z}$ and $r>0$ one has

$$
\left.\left.\mathrm{IC}_{0}\right|_{\text {Shatz }_{\tilde{G}}^{r}} \widetilde{\rightarrow} \mathrm{IC}_{r}\left[\frac{2 r}{n}-1\right]\right|_{\text {Shatz }_{\tilde{G}}^{r}}
$$

Proof For $\left(M, \mathcal{U}_{G}\right) \in \operatorname{Shat}_{\tilde{G}}^{0}$ the fibre of $\overline{\mathfrak{p}}: \overline{\operatorname{Bun}}_{B}^{0} \rightarrow \operatorname{Bun}_{G}$ over $M$ is isomorphic to $\mathbb{P}^{1}$. So, $\operatorname{Eis}^{0}(\mathcal{K}) \widetilde{\rightarrow} \mathrm{IC}_{0}[1] \oplus \mathrm{IC}_{0}[-1]$ over $\operatorname{Shat}_{\tilde{G}}^{0}$. For $d>0$ and a point $\left(\mathcal{E} \subset M, \mathcal{U}_{G}\right) \in S h a t z_{\tilde{G}}^{d}$ the fibre of $\overline{\mathfrak{p}}: \overline{\operatorname{Bun}}_{G} \rightarrow \operatorname{Bun}_{G}$ over $M$ identifies with $X^{(d)}$. To $D \in X^{(d)}$ there corresponds 
$(\mathcal{E}(-D) \subset M) \in \overline{\mathrm{Bun}}_{B}^{0}$. Now arguing as in Proposition $\mathbf{7 . 4}$, one shows that the $*$-restriction $\left.\operatorname{Eis}^{0}(\mathcal{K})\right|_{\text {Shat }} z_{\tilde{G}}$ vanishes unless $d \in n \mathbb{Z}$. For $d \in n \mathbb{Z}$ we get

$$
\left.\operatorname{Eis}^{0}(\mathcal{K})\right|_{\text {Shatz }} \underset{\tilde{G}}{\widetilde{\rightarrow}} \tilde{q}^{*} \mathcal{K} \otimes \mathrm{R} \Gamma\left(X^{\left(\frac{d}{n}\right)}, \overline{\mathbb{Q}}_{\ell}\right)\left[-2 d-2+2 \frac{d}{n}\right]
$$

The codimension of $S h a t z^{d}$ in $\operatorname{Bun}_{G}$ is $2 d-1$. It follows that $\left.\operatorname{Eis}^{0}(\mathcal{K})\right|_{\operatorname{Shatz}_{\tilde{G}}^{d}}$ is placed in perverse degrees $\leq 0$, and its 0 -th perverse cohomology sheaf is isomorphic to $\mathrm{IC}_{d}$.

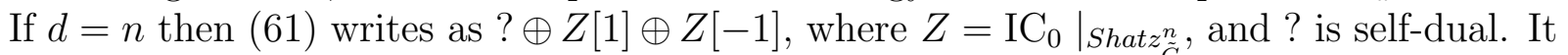
follows that $\left.\left.\mathrm{IC}_{0}\right|_{\text {Shat }} ^{n} \underset{\tilde{G}}{\rightarrow} \mathrm{IC}_{n}[1]\right|_{\text {Shatz }} ^{n}$ and $?=0$.

For $d=2 n$ the complex (61) writes as ? $\oplus Z[1] \oplus Z[-1]$, where $Z=\left.\mathrm{IC}_{0}\right|_{\text {Shatz }}$, and ? is self-dual. It follows that $?=\mathrm{IC}_{2 n}$ and $\left.\mathrm{IC}_{0}\right|_{\text {Shatz }} ^{2 n} \underset{\tilde{G}}{\rightarrow} \mathrm{IC}_{2 n}[3]$.

For $d=3 n$ the complex (61) writes as

$$
\left.? \oplus Z[1] \oplus Z[-1] \oplus \mathrm{IC}_{2 n}\right|_{S h a t z_{\tilde{G}}^{d}}
$$

where ? is self-dual, and $Z=\left.\mathrm{IC}_{0}\right|_{\text {Shatz }_{\tilde{G}}^{d}}$. By Lemma $7.3,\left.\mathrm{IC}_{2 n}\right|_{\text {Shat }} ^{\tilde{G}} \underset{\rightarrow}{\rightarrow} \mathrm{IC}_{3 n}[2]$ over Shat $z_{\tilde{G}}^{d}$. It follows that $?=\mathrm{IC}_{d}$ and $\left.\mathrm{IC}_{0}\right|_{\text {Shatz }} \tilde{G}_{\tilde{G}} \underset{\rightarrow}{\rightarrow} \mathrm{IC}_{3 n}[5]$. Continuing this, our claim easily follows by induction.

Remark 7.6. If $n=2$ then $\mathrm{IC}_{0}=$ Aut $_{g}$ and $\mathrm{IC}_{1}=$ Aut $_{s}$ in the notations of [20] and ([18], Appendix A). These are the direct summands of the theta-sheaf Aut. Our description of the fibres of $\mathrm{IC}_{0}$ and $\mathrm{IC}_{1}$ in Lemmas 7.3 and 7.4 extends ([20], Theorem 1).

Recall the Hecke functors for $G$ defined in Section 3.2. For $\nu \in \Lambda^{\sharp,+}$ we set ${ }_{x} \mathrm{H}_{G}^{\nu}=$ $\left(\text { id } \times i_{x}\right)^{*} \mathrm{H}_{G}^{\nu}[-1]$, where $i_{x}:$ Spec $k \rightarrow X$ is the point $x$, and $i d \times i_{x}: \widetilde{\operatorname{Bun}}_{G} \rightarrow \widetilde{\operatorname{Bun}}_{G} \times X$.

Lemma 7.5. Let $\nu=e \alpha, d>0$ with $d \in e \mathbb{Z}$.

(1) Assume $n$ even. For $d \geq n$ one has ${ }_{x} \mathrm{H}_{G}^{\nu} \mathrm{IC}_{d} \underset{\rightarrow}{\rightarrow} \mathrm{IC}_{d+e} \oplus \mathrm{IC}_{d-e}$. Besides,

$$
{ }_{x} \mathrm{H}_{G}^{\nu} \mathrm{IC}_{e} \underset{\rightarrow}{\rightarrow} \mathrm{IC}_{0}[1] \oplus \mathrm{IC}_{0}[-1]
$$

(2) Assume $n$ odd. For $d \geq 2$ one has ${ }_{x} \mathrm{H}_{G}^{\nu} \mathrm{IC}_{d} \widetilde{\rightarrow} \mathrm{IC}_{d+n} \oplus \mathrm{IC}_{d} \oplus \mathrm{IC}_{d-n}$. Besides,

$$
{ }_{x} \mathrm{H}_{G}^{\nu} \mathrm{IC}_{n} \stackrel{\rightarrow}{\rightarrow} \mathrm{IC}_{0}[1] \oplus \mathrm{IC}_{0}[-1] \oplus \mathrm{IC}_{2 n}
$$

Proof By Proposition 7.4 and Lemma 7.4, $\operatorname{Eis}^{d}(\mathcal{K}) \widetilde{\rightarrow} \operatorname{Eis}^{d+n}(\mathcal{K}) \oplus \mathrm{IC}_{d}$ and

$$
\operatorname{Eis}^{0}(\mathcal{K}) \stackrel{\rightarrow}{\rightarrow} \mathrm{IC}_{0}[1] \oplus \mathrm{IC}_{0}[-1] \oplus \operatorname{Eis}^{2 n}(\mathcal{K})
$$

Write $\widetilde{\operatorname{Bun}}_{T}^{d}$ for the component of $\widetilde{\operatorname{Bun}}_{T}$ classifying $(\mathcal{E}, \mathcal{U})$ with $\operatorname{deg} \mathcal{E}=d$. Applying Theorem 2.2 to the complex $\left.\mathcal{K}\right|_{\widetilde{B u n}_{T}^{d}}$ one gets the following.

1) Assume $n$ even. One has

$$
{ }_{x} \mathrm{H}_{G}^{\nu} \operatorname{Eis}^{d}(\mathcal{K}) \stackrel{\rightarrow}{\rightarrow} \operatorname{Eis}^{d+e}(\mathcal{K}) \oplus \operatorname{Eis}^{d-e}(\mathcal{K})
$$


and ${ }_{x} \mathrm{H}_{G}^{\nu} \operatorname{Eis}^{d+n}(\mathcal{K}) \underset{\rightarrow}{\rightarrow} \operatorname{Eis}^{d+n+e}(\mathcal{K}) \oplus \operatorname{Eis}^{d+n-e}(\mathcal{K})$. For $d \geq n$ this implies

$$
{ }_{x} \mathrm{H}_{G}^{\nu} \mathrm{IC}_{d} \widetilde{\rightarrow} \mathrm{IC}_{d+e} \oplus \mathrm{IC}_{d-e}
$$

Besides, ${ }_{x} \mathrm{H}_{G}^{\nu} \mathrm{IC}_{e} \underset{\rightarrow}{\rightarrow} \mathrm{IC}_{0}[1] \oplus \mathrm{IC}_{0}[-1]$.

2) Assume $n$ odd. One has

$$
{ }_{x} \mathrm{H}_{G}^{\nu} \operatorname{Eis}^{d}(\mathcal{K}) \stackrel{\rightarrow}{\rightarrow} \operatorname{Eis}^{d+n}(\mathcal{K}) \oplus \operatorname{Eis}^{d}(\mathcal{K}) \oplus \operatorname{Eis}^{d-n}(\mathcal{K})
$$

and ${ }_{x} \mathrm{H}_{G}^{\nu} \operatorname{Eis}^{d+n}(\mathcal{K}) \widetilde{\rightarrow} \operatorname{Eis}^{d+2 n}(\mathcal{K}) \oplus \operatorname{Eis}^{d+n}(\mathcal{K}) \oplus \operatorname{Eis}^{d}(\mathcal{K})$. For $d \geq 2 n$ this implies

$$
{ }_{x} \mathrm{H}_{G}^{\nu} \mathrm{IC}_{d} \widetilde{\rightarrow} \mathrm{IC}_{d+n} \oplus \mathrm{IC}_{d} \oplus \mathrm{IC}_{d-n}
$$

Besides, ${ }_{x} \mathrm{H}_{G}^{\nu} \mathrm{IC}_{n} \widetilde{\rightarrow} \mathrm{IC}_{0}[1] \oplus \mathrm{IC}_{0}[-1] \oplus \mathrm{IC}_{2 n}$.

Proposition 7.5. Let $\nu=e \alpha$.

(1) If $n$ is even $\mathcal{A}_{\varepsilon}^{\nu}$ is the extension by zero from $\widetilde{\mathrm{Gr}}_{G}^{\nu}$.

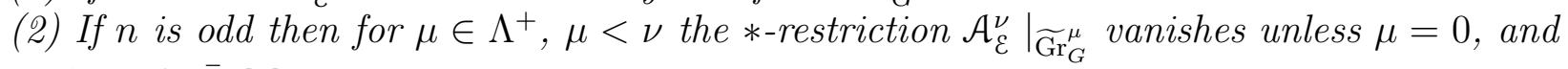
$\left.\mathcal{A}_{\varepsilon}^{\nu}\right|_{\mathrm{Gr}_{G}^{0}} \widetilde{\rightarrow} \overline{\mathbb{Q}}_{\ell}[2]$.

Proof In the proof we use some notations from [9]. Let $\mathcal{O}=k[[t]]$. Recall that $\mathbb{A}^{e} \stackrel{\Im}{\rightarrow} \operatorname{Gr}_{B}^{0} \cap \overline{\mathrm{Gr}}_{G}^{\nu}$ via the map sending $f=b_{e} t^{-e}+b_{e-1} t^{1-e}+\ldots+b_{1} t^{-1}$ with $b_{i} \in \mathbb{A}^{1}$ to

$$
\left(\begin{array}{ll}
1 & f \\
0 & 1
\end{array}\right) G(\mathcal{O})
$$

The open subscheme $\operatorname{Gr}_{B}^{0} \cap \mathrm{Gr}_{G}^{\nu} \subset \mathrm{Gr}_{B}^{0} \cap \overline{\mathrm{Gr}}_{G}^{\nu}$ is given by $b_{e} \neq 0$. In the notations of ([9], Lemma 4.2) one has $F_{T}^{0}\left(\mathcal{A}_{\varepsilon}^{\nu}\right)=0$ for $n$ even, and $F_{T}^{0}\left(\mathcal{A}_{\varepsilon}^{\nu}\right) \widetilde{\rightarrow} \overline{\mathbb{Q}}_{\ell}$ for $n$ odd.

For $\mu \in \Lambda^{+}, \mu<\nu$ the $*$-restriction $\left.\mathcal{A}_{\varepsilon}^{\nu}\right|_{\widetilde{G r}_{G}^{\mu}}$ vanishes unless $\mu=0$. Indeed, if $\mu \in \Lambda^{+}$, $\mu<\nu$ and $\mu \in \Lambda^{\sharp}$ then $\mu=0$. One has the diagram

$$
{\widetilde{\mathrm{Gr}_{T}}}_{T}^{0} \stackrel{\mathfrak{t}_{B}}{\leftarrow}{\widetilde{\mathrm{Gr}_{B}}}_{B}^{0} \stackrel{\mathfrak{s}_{B}}{\rightarrow} \widetilde{\mathrm{Gr}}_{G}
$$

In the notations of ([9], Lemma 4.2) we see that $a_{B, 0}^{*} \mathcal{A}_{\varepsilon}^{\nu}$ is not constant (resp., is constant) over $\operatorname{Gr}_{B}^{0} \cap \mathrm{Gr}_{G}^{\nu}$ for $n$ even (resp., for $n$ odd).

Calculate $\left(\mathfrak{t}_{B}\right) \mathfrak{s}_{B}^{*} \mathcal{A}_{\varepsilon}^{\nu}$ using the stratification of $\operatorname{Gr}_{B}^{0} \cap{\overline{\mathrm{Gr}_{G}}}_{G}^{\nu}$ by the locally closed subschemes $\operatorname{Gr}_{B}^{0} \cap \operatorname{Gr}_{G}^{\mu}$ with $\mu \in \Lambda^{+}, \mu \leq \nu$. For $n$ even the contribution of $\widetilde{\mathrm{Gr}}_{B}^{0} \cap \widetilde{\mathrm{Gr}}_{G}^{\nu}$ to this direct

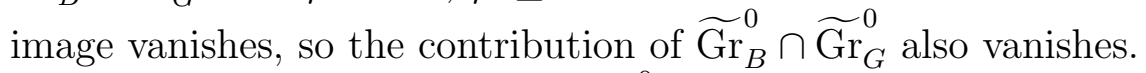

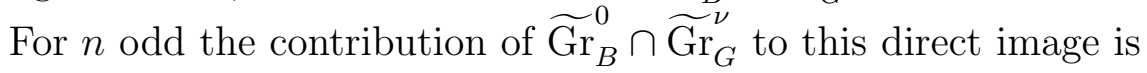

$$
\mathrm{R} \Gamma_{c}\left(\mathbb{G}_{m} \times \mathbb{A}^{n-1}, \overline{\mathbb{Q}}_{\ell}[2 n]\right) \widetilde{\rightarrow} \overline{\mathbb{Q}}_{\ell}[1] \oplus \overline{\mathbb{Q}}_{\ell}
$$

Since $F_{T}^{0}\left(\mathcal{A}_{\varepsilon}^{\nu}\right) \widetilde{\widetilde{T}} \overline{\mathbb{Q}}_{\ell}$, and $\left.\mathcal{A}_{\varepsilon}^{\nu}\right|_{\widetilde{G r}_{G}^{0}}$ is placed in strictly negative degrees, our claim follows from the exact triangle $\left.\left(\overline{\mathbb{Q}}_{\ell}[1] \oplus \overline{\mathbb{Q}}_{\ell}\right) \rightarrow \overline{\mathbb{Q}}_{\ell} \rightarrow \mathcal{A}_{\varepsilon}^{\nu}\right|_{\mathrm{Gr}_{G}^{0}}$.

Write ${ }_{x} \mathcal{H}_{\tilde{G}}^{\nu}$ for the preimage of ${ }_{x} \mathcal{H}_{G}^{\nu}$ in ${ }_{x} \mathcal{H}_{\tilde{G}}$. 
Lemma 7.6. Let $\nu=e \alpha$. For a point $\left(M, M^{\prime}, \beta, x\right) \in \mathcal{H}_{G}^{\nu}$ one has canonically

$$
\frac{\operatorname{det} \mathrm{R} \Gamma\left(X, M^{\prime}\right)}{\operatorname{det} \mathrm{R} \Gamma(X, M)} \underset{\rightarrow}{\rightarrow}\left(\left(M((e-1) x)+M^{\prime}\right) / M((e-1) x)\right)^{\otimes 2 e} \otimes \Omega_{x}^{e(e-1)}
$$

Here $\left.\operatorname{dim}_{k} M((e-1) x)+M^{\prime}\right) / M((e-1) x)=1$. There is an isomorphism

$$
\kappa:{ }_{x} \mathcal{H}_{\tilde{G}}^{\nu} \widetilde{\rightarrow}\left(\widetilde{\operatorname{Bun}}_{G} \times{ }_{\operatorname{Bun}_{G} x} \mathcal{H}_{G}^{\nu}\right) \times B\left(\mu_{N}\right),
$$

where we used $h_{G}^{\overleftarrow{ }}$ in the fibred product, and the projection to the first term corresponds to $\tilde{h}_{G}^{\leftarrow}:{ }_{x} \mathcal{H}_{\tilde{G}}^{\nu} \rightarrow \widetilde{\operatorname{Bun}}_{G}$

Proof The symplectic form on $M$ yields a nondegenerate pairing between the $k$-vector spaces $\left(M+M^{\prime}\right) / M$ and $\left(M+M^{\prime}\right) / M^{\prime}$. So,

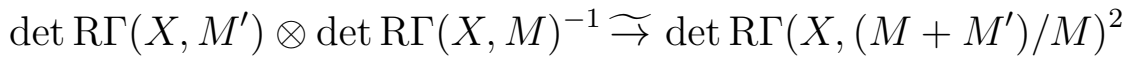

The first claim follows now from the natural isomorphism

$$
\operatorname{det} \mathrm{R} \Gamma\left(X,\left(M+M^{\prime}\right) / M\right) \stackrel{\Im}{\rightarrow}\left(\left(M((e-1) x)+M^{\prime}\right) / M((e-1) x)\right)^{\otimes e} \otimes \Omega_{x}^{e(e-1) / 2}
$$

A point of ${ }_{x} \mathcal{H}_{\tilde{G}}^{\nu}$ is given by a collection $\left(M, M^{\prime}, \beta\right) \in{ }_{x} \mathcal{H}_{G}^{\nu}$ and the lines $\mathcal{U}, \mathcal{U}^{\prime}$ together with

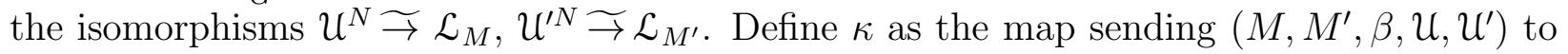
$\left(M, M^{\prime}, \beta, \mathcal{U}, \mathcal{U}_{0}\right)$, where

$$
\mathcal{U}=\mathcal{U}^{\prime} \otimes \mathcal{U}_{0} \otimes\left(\left(M((e-1) x)+M^{\prime}\right) / M((e-1) x)\right)^{\otimes \frac{2 e}{n}} \otimes \mathcal{E}_{X, x}^{\frac{2 e(e-1)}{n}}
$$

For $n$ odd the line $\Omega_{x}^{\frac{e(e-1)}{n}} \underset{\rightarrow}{\rightarrow} \mathcal{E}_{X, x}^{\frac{2 e(e-1)}{n}}$ does not depend on the choice of $\mathcal{E}_{X}$.

For $\mathcal{M} \in \mathrm{D}_{\zeta}\left(\widetilde{\operatorname{Bun}}_{G}\right)$ the contribution of ${ }_{x} \mathcal{H}_{\tilde{G}}^{\nu}$ to ${ }_{x} \mathrm{H}_{G}^{\nu}(\mathcal{M})$ now writes as

$$
\left(\tilde{h}_{G}^{\leftarrow}\right) !\left(\left(\tilde{h}_{G}\right)^{*} \mathcal{M} \otimes \kappa^{*} \mathcal{L}_{\zeta}\right)[2 e]
$$

for the diagram $\widetilde{\operatorname{Bun}_{G}} \stackrel{\tilde{h}_{G}^{\leftarrow}}{\leftarrow}{ }_{x} \mathcal{H}_{\tilde{G}}^{\nu} \stackrel{\tilde{h} \vec{G}}{\rightarrow \operatorname{Bun}_{G}}$. For $n$ odd the contribution of ${ }_{x} \mathcal{H}_{\tilde{G}}^{0}$ to ${ }_{x} \mathrm{H}_{G}^{\nu}(\mathcal{M})$ is $\mathcal{M}[2]$.

Theorem 7.1. Let $\nu=e \alpha$.

(1) For $n$ even one has ${ }_{x} \mathrm{H}_{G}^{\nu} \mathrm{IC}_{0} \underset{\rightarrow}{\rightarrow} \mathrm{IC}_{e}[1] \oplus \mathrm{IC}_{e}[-1]$.

(2) For $n$ odd one has

$$
{ }_{x} \mathrm{H}_{G}^{\nu} \mathrm{IC}_{0} \underset{\rightarrow}{\rightarrow} \mathrm{IC}_{0}[2] \oplus \mathrm{IC}_{0} \oplus \mathrm{IC}_{0}[-2]
$$

Proof Recall that ${ }_{x} \mathrm{H}_{G}^{\nu}$ is given by a version of (6) with $x$ fixed.

(1) By Lemma 3.6 and Remark 7.5, ${ }_{x} \mathrm{H}_{G}^{\nu} \mathrm{IC}_{0} \in \mathrm{D}_{\zeta,-}\left(\widetilde{\mathrm{Bun}_{G}}\right)$. This implies that the *restriction $\left.\left({ }_{x} \mathrm{H}_{G}^{\nu} \mathrm{IC}_{0}\right)\right|_{S h a t z} z_{\tilde{G}}$ vanishes unless $i \in e+n \mathbb{Z}$.

Let $i \geq 0, i \in e+n \mathbb{Z}$ and $M \in S h a t z^{i}$. Let $Y$ denote the fibre of $h_{G}^{\overleftarrow{ }}:{ }_{x} \mathcal{H}_{G}^{\nu} \rightarrow \operatorname{Bun}_{G}$ over $M$. Write $\mathbb{P}\left(M_{x}\right)$ for the projective space of lines in $M(e x) / M((e-1) x)$. We have a map $\eta: Y \rightarrow \mathbb{P}\left(M_{x}\right)$ sending $M^{\prime}$ to the line $\left(M^{\prime}+M((e-1) x)\right) / M((e-1) x)$. Each fibre of $\eta$ identifies with $\mathbb{A}^{2 e-1}$. Denote by $S$ the $*$-fibre of ${ }_{x} \mathrm{H}_{G}^{\nu} \mathrm{IC}_{0}$ at $M$. 
For $d \geq 0$ with $d \in n \mathbb{Z}$ let $Y_{d} \subset Y$ be the locally closed subscheme given by $M^{\prime} \in S h a t z^{d}$. Since $i>0, M$ has a canonical $B$-structure given by $(\mathcal{E} \subset M)$ with $\operatorname{deg} \mathcal{E}=i, \mathcal{E} \in \operatorname{Bun}_{1}$.

If $i=e$ then $Y_{0} \widetilde{\rightarrow} \mathbb{A}^{n}$, and the contribution of this locus to $S$ is $\overline{\mathbb{Q}}_{\ell}[-3-n]$. Besides, $Y_{n} \widetilde{\rightarrow} \mathrm{Gr}_{B}^{-e \alpha} \cap \mathrm{Gr}_{G}^{e \alpha} \widetilde{\rightarrow}$ Spec $k$. So, the contribution of $Y_{n}$ to $S$ is $\overline{\mathbb{Q}}_{\ell}[-1-n]$. We see that the $*$-restriction of ${ }_{x} \mathrm{H}_{G}^{\nu} \mathrm{IC}_{0}$ to $S h a t z^{e}$ identifies with $\mathrm{IC}_{e}[1] \oplus \mathrm{IC}_{e}[-1]$.

Assume $i=e+b n$ with $b>0, d \geq 0, d \in n \mathbb{Z}$. Then $Y_{d} \subset Y$ is not empty only for $d=n b$ or $d=n(b+1)$. One has $Y_{n b} \widetilde{\rightarrow} \mathrm{Gr}_{B}^{e \alpha} \cap \mathrm{Gr}_{G}^{e \alpha} \widetilde{\rightarrow} \mathbb{A}^{n}$. The contribution of this locus to $S$ is

$$
\left.\overline{\mathbb{Q}}_{\ell}[-3-2 b n+2 b-n] \stackrel{\rightarrow}{\rightarrow} \mathrm{IC}_{e}[-1]\right|_{M}
$$

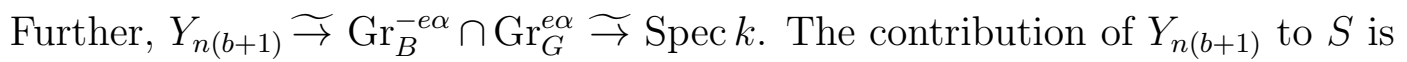

$$
\left.\overline{\mathbb{Q}}_{\ell}[-1-2 b n+2 b-n] \stackrel{\rightarrow}{\rightarrow} \mathrm{IC}_{e}[1]\right|_{M}
$$

Part (1) follows.

(2) The case $n=1$ is well-known, assume $n>1$. We denote by $\left(M, M^{\prime}, \beta\right)$ a point of $\overline{\mathcal{H}}_{G}^{\nu}$. Let $i \geq 0, i \in n \mathbb{Z}$ and $M \in S h a t z^{i}$. Let $\bar{Y}$ denote the fibre of $h_{G}^{\overleftarrow{Y}}:{ }_{x} \overline{\mathcal{H}}_{G}{ }_{G} \rightarrow \operatorname{Bun}_{G}$ over $M$. Let $Y \subset \bar{Y}$ be the preimage of ${ }_{x} \mathcal{H}_{G}^{\nu}$ in $\bar{Y}$.

For $d \geq 0, d \in n \mathbb{Z}$ let $Y_{d} \subset Y$ (resp., $\bar{Y}_{d} \subset \bar{Y}$ ) denote the locally closed subscheme given by $M^{\prime} \in S h a t z^{d}$. Write $S$ for the fibre of ${ }_{x} \mathrm{H}_{G}^{\nu} \mathrm{IC}_{0}$ at $M$. As above, we have a map $\eta: Y \rightarrow \mathbb{P}\left(M_{x}\right)$, each fibre identifies with $\mathbb{A}^{2 n-1}$.

First, assume $i=0$. Then only $\bar{Y}_{n}=Y_{n}$ and $\bar{Y}_{0}$ contribute to $S$. There is a section $\mathbb{P}\left(M_{x}\right) \rightarrow Y$ of $\eta$, whose image identifies with $Y_{n}$. Any local system on $\mathbb{P}^{1}$ is constant. So, the contribution of $Y_{n}$ to $S$ is $\mathrm{R} \Gamma\left(\mathbb{P}^{1}, \overline{\mathbb{Q}}_{\ell}[-1]\right) \stackrel{\sim}{\rightarrow} \overline{\mathbb{Q}}_{\ell}[-1] \oplus \overline{\mathbb{Q}}_{\ell}[-3]$.

The scheme $\bar{Y}_{0}$ can be written as the subscheme of $\operatorname{Gr}_{G}$ of points of the form $A G(\mathcal{O})$, where

$$
A=\left(\begin{array}{cc}
1+a_{1} & a_{2} \\
a_{3} & 1+a_{4}
\end{array}\right) \in G\left(k\left[t^{-1}\right]\right)
$$

with $a_{i} \in k\left[t^{-1}\right]$ of degree $\leq n$ in $t^{-1}$. In particular, $G$ acts on $\bar{Y}_{0}$. This action commutes with the loop rotations $\mathbb{G}_{m} \subset \operatorname{Aut}(\mathcal{O})$ action on $\bar{Y}_{0}$. The scheme $\bar{Y}_{0}$ can equally be seen as the scheme classifying matrices $A$ as above with $a_{i}$ of the form $b_{n, i} t^{-n}+\ldots+b_{1, i} t^{-1} \in k\left[t^{-1}\right]$ for all $i$. In the latter form the action of $G$ is given by the conjugation.

Recall the formulas (62) and (6) . The group $\mathbb{G}_{m} \subset \operatorname{Aut}(\mathcal{O})$ of loop rotations acts on $\bar{Y}_{0}$ and contracts it to the point $M \in \bar{Y}_{0}$. The complex $\left(\tilde{h}_{G}\right)^{*} \mathrm{IC}_{0} \otimes \mathrm{IC}^{\nu}$ is monodromic with respect to this action. Let $i_{0}:$ Spec $k \rightarrow \bar{Y}_{0}$ denote the point $M$. By ([2], Lemma 5.3) we get

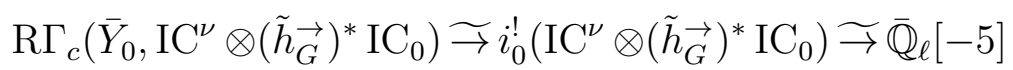

It follows that over $\operatorname{Shatz}^{0}$ one has

$$
{ }_{x} \mathrm{H}_{G}^{\nu} \mathrm{IC}_{0} \underset{\rightarrow}{\rightarrow} \mathrm{IC}_{0}[2] \oplus \mathrm{IC}_{0} \oplus \mathrm{IC}_{0}[-2]
$$

Let now $i>0, i \in n \mathbb{Z}$. Then $M$ has a distinguished $B$-structure given by the unique subbundle of degree $i$. Then only $\bar{Y}_{n+i}, \bar{Y}_{i}, \bar{Y}_{i-n}$ may contribute to $S$. Note that $\bar{Y}_{n+i}=$ $Y_{n+i}$ is the point scheme. Its contribution to $S$ is $\overline{\mathbb{Q}}_{\ell}\left[-1-2 i+\frac{2 i}{n}\right]$. One has $Y_{i-n}=$ $\bar{Y}_{i-n} \underset{\rightarrow}{\rightarrow} \mathrm{Gr}_{B}^{\nu} \cap \mathrm{Gr}_{G}^{\nu} \widetilde{\rightarrow} \mathbb{A}^{2 n}$. So, the contribution of $\bar{Y}_{i-n}$ to $S$ is $\overline{\mathbb{Q}}_{\ell}\left[-5-2 i+\frac{2 i}{n}\right]$. 
Finally, $\bar{Y}_{i} \widetilde{\rightrightarrows} \mathrm{Gr}_{B}^{0} \cap \overline{\mathrm{Gr}}_{G}^{\nu} \widetilde{\rightrightarrows} \mathbb{A}^{n}$. To calculate the contribution of $\bar{Y}_{i}$ to $S$, argue as in Proposition 7.5. In the notations of ([9], Lemma 4.2), the contribution of $\bar{Y}_{i}$ to $S$ identifies with

$$
F_{T}^{0}\left(\mathcal{A}_{\varepsilon}^{\nu}\right)\left[-3-2 i+\frac{2 i}{n}\right] \widetilde{\rightarrow} \overline{\mathbb{Q}}_{\ell}\left[-3-2 i+\frac{2 i}{n}\right] \stackrel{\rightarrow}{\rightarrow}\left(\mathrm{IC}_{0}\right)_{M}
$$

Using Lemma 7.4 we see that $S$ is isomorphic to the $*$-restriction of $\mathrm{IC}_{0}[2] \oplus \mathrm{IC}_{0} \oplus \mathrm{IC}_{0}[-2]$ to $M$. We are done.

\section{Appendix A. Proof of Theorem 2.6}

A.1. Assume $k=\mathbb{F}_{q}$. A version of Corollary 5.1 holds also in this case. It is understood that the Tate twists are recovered in the corresponding formulas. Take $P=B$, so $M=T$. Let $\theta \in \Lambda^{\text {pos }}$. In this case the stack $\operatorname{Mod}_{\tilde{M}}^{+, \theta}$ defined in Section 5.1 classifies $\bar{D} \in X^{\theta}$ and a line $\mathcal{U}$ together with $\mathcal{U}^{N} \underset{\rightarrow}{\rightarrow} \mathcal{L}_{\mathcal{F}_{T}^{0}(-\bar{D})}$.

The stack $\widetilde{\operatorname{Mod}_{\operatorname{Bun}_{T}}^{+, \theta}}$ from Section 5.2.1 classifies $\left(\bar{D} \in X^{\theta}, \mathcal{F}_{T}^{\prime} \in \operatorname{Bun}_{T}, \mathcal{U}, \mathcal{U}^{\prime}\right)$, where $\mathcal{U}, \mathfrak{U}^{\prime}$

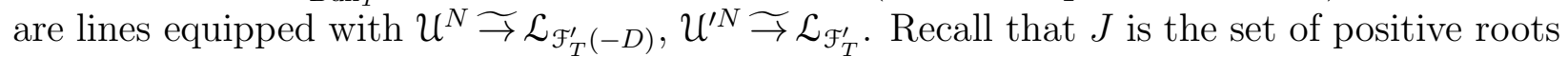
of $\check{G}_{n}$ for $\check{B}_{n}$.

For $\mathfrak{B}(\theta)=\sum_{\nu \in J} n_{\nu} \nu \in \Lambda_{G, B}^{\text {pos,pos }}$ the perverse sheaf $\operatorname{IC}_{\mathrm{Bun}_{T}, \zeta}^{\mathfrak{B}(\theta)}$ on $X^{\mathfrak{B}(\theta)} \times_{X^{\theta}} \widetilde{\operatorname{Mod}}_{\mathrm{Bun}_{T}}^{+, \theta}$ is a rank one shifted local system. Consider the diagram

$$
X^{\mathfrak{B}(\theta)} \times \widetilde{\operatorname{Bun}}_{T} \stackrel{f}{\leftarrow} X^{\mathfrak{B}(\theta)} \times{ }_{X^{\theta}} \widetilde{\operatorname{Mod}_{\mathrm{Bun}_{T}}^{+, \theta}} \stackrel{h}{\rightarrow} \widetilde{\operatorname{Bun}_{T}}
$$

where $f$ sends $D \in X^{\mathfrak{B}(\theta)}$ with image $\bar{D} \in X^{\theta},\left(\bar{D}, \mathcal{F}_{T}^{\prime}, \mathcal{U}, \mathcal{U}^{\prime}\right) \in \widetilde{\operatorname{Mod}^{+, \theta}} \frac{\operatorname{Bun}_{T}}{\text { to }}\left(D, \mathcal{F}_{T}^{\prime}, \mathcal{U}^{\prime}\right)$, and $h$ sends the above point to $\left(\mathcal{F}_{T}^{\prime}(-\bar{D}), \mathcal{U}\right) \in \widetilde{\operatorname{Bun}}_{T}$.

Recall that $X^{\mathfrak{B}(\theta)}=\prod_{\nu \in J} X^{\left(n_{\nu}\right)}$ and $|\mathfrak{B}(\theta)|$ is the dimension of $X^{\mathfrak{B}(\theta)}$. One has

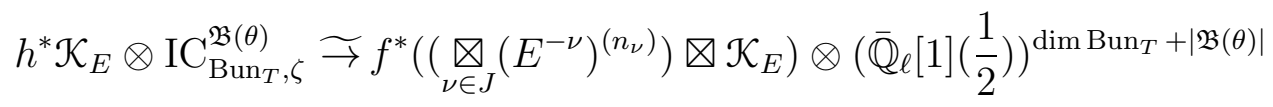

So, by Corollary 5.1, the contribution of the stratum ${ }_{\theta} \widetilde{\operatorname{Bun}}_{\tilde{B}}$ to $\operatorname{Funct}\left(\operatorname{Eis}\left(\mathcal{K}_{E}^{\mu}\right)\right)$ is

$$
\operatorname{Funct}\left(\operatorname{Eis}^{\prime}\left(\mathcal{K}_{E}^{\mu-\theta}\right)\right) \prod_{\nu \in J} \operatorname{Tr}\left(\operatorname{Fr}, \operatorname{R} \Gamma\left(X^{\left(n_{\nu}\right)},\left(E^{-\nu}\right)^{\left(n_{\nu}\right)}\right) \otimes \overline{\mathbb{Q}}_{\ell}\left(n_{\nu}\right)\right)
$$

Theorem 2.6 is proved.

Acknowledgements. The author is grateful to V. Lafforgue and M. Finkelberg for many fruitful discussions that helped us to overcome some difficulties on the way. We thank D. Gaitsgory for answering our questions. It is a pleasure to thank the organizers of the conference 'Automorphic forms and harmonic analysis on covering groups' (The American Institute of Mathematics, Palo Alto, 2013), which stimulated our work. The author was supported by the ANR project ANR-13-BS01-0001-01. 


\section{REFERENCES}

[1] R. Bezrukavnikov, M. Finkelberg, V. Schechtman, Factorizable Sheaves and Quantum Groups, Lecture Notes in Math., Vol. 1691 (1998)

[2] A. Braverman, M. Finkelberg, D. Gaitsgory, I. Mirkovic, Intersection cohomology of Drinfeld compactifications, Sel. math., New ser. 8 (2002), 381-418; Erratum: Sel. math., New ser. 10 (2004), p. 429-430

[3] A. Braverman, D. Gaitsgory, Geometric Eisenstein series, Inv. Math. 150 (2002), 287-384

[4] B. Brubaker, D. Bump, S. Friedberg, Eisenstein Series, Crystals and Ice, Notices of the AMS (2011), p. 1563-1571

[5] J.-L. Brylinski, P. Deligne, Central extensions of reductive groups by $K_{2}$, Publ. Math. de l'IHES, Vol. 94 (2001), p. 5-85

[6] D. Bump, Introduction: Multiple Dirichlet Series, preprint available at http://sporadic.stanford.edu/bump/multiple.pdf

[7] P. Deligne, Seminaire de Géométrie Algébrique du Bois-Marie $S G A 4 \frac{1}{2}$, Cohomologie Etale, Lecture Notes in Math. 569 (1977)

[8] P. Deligne, Le déterminant de la cohomologie, Comtemp. Math., Vol 67 (1987), p. 93-177

[9] M. Finkelberg, S. Lysenko, Twisted geometric Satake equivalence, J. Inst. Math. Jussieu, 9 (2010), no. 4, p. $719-739$

[10] M. Finkelberg, V. Schechtman, Microlocal approach to Lusztig's symmetries, arXiv:1401.5885

[11] D. Gaitsgory, Central elements in the affine Hecke algebra via nearby cycles, Invent. Math. 144 (2001), p. $253-280$

[12] D. Gaitsgory, Twisted Whittaker model and factorizable sheaves, Sel. Math., New Ser. 13, No. 4, (2008), p. $617-659$

[13] D. Gaitsgory, Quantum Langlands Correspondence, arXiv:1601.05279

[14] W. T. Gan, F. Gao, The Langlands-Weissman program for Brylinski-Deligne extensions, arXiv:1409.4039

[15] F. Gao, The Gindikin-Karpelevich formula and constant terms of Eisenstein series for Brylinski-Deligne extensions, PhD. thesis, University of Singapore (2014)

[16] Y. Laszlo, M. Olsson, The six operations for sheaves on Artin stacks II: adic coefficients, Publ. Math. IHES, Vol. 107, No. 1 (2008), p. 169-210

[17] S. Lysenko, Twisted geometric Langlands correspondence for a torus, IMRN, 18, (2015), 8680-8723

[18] S. Lysenko, Geometric Whittaker models and Eisenstein series for $\mathrm{Mp}_{2}$, arXiv:1211.1596

[19] S. Lysenko, Geometric Waldspurger periods II, arXiv:1308.6531

[20] S. Lysenko, Moduli of metaplectic bundles on curves and Theta-sheaves, Ann. Scient. Ecole Norm. Sup., 4 série, t. 39 (2006), p. 415-466

[21] S. Lysenko, Twisted geometric Satake equivalence: reductive case, arXiv:1411.6782

[22] S. Lysenko, Twisted Whittaker models for metaplectic groups, arXiv:1509.02433

[23] C. Moeglin, J.-L. Waldspurger, Spectral decomposition and Eisenstein series : une paraphrase de l'écriture, Cambridge University Press (1995)

[24] G. Savin, On unramified representations of covering groups, J. reine angew. Math. 566 (2004), p. 111-134

[25] V. Schechtman, Dualité de Langlands quantique, Ann. Fac. Sci. Toulouse Math., XXIII (2014), p. 129-158.

[26] S. Schieder, The Harder-Narasimhan stratication of Bun $_{G}$ via Drinfeld's compactifications, arXiv:1212.6814

[27] Springer, Reductive Groups, Proc. Symp. Pure Math, vol. 33, part I (1979), p. 1-27

[28] M. Weissman, L-groups and parameters for covering groups, arXiv:1507.01042

[29] M. Weissman, Split metaplectic groups and their L-groups, J. fr die reine und angew. Math. (2014), Issue 696, p. 89141 